\title{
ENVIRONMENTAL PROTECTION DEPARTMENT'S WELL INVENTORY (U) (through the second quarter of 1991)
}

Prepared By

Environmental Protection. Department

Westinghouse Savannah River Company

Aiken, SC

and

Exploration Resources, Inc.

Athens, GA

Publication Date: October 1991

Maps Prepared By

Exploration Resources, Inc.

Athens, GA

Westinghouse Savannah River Company

Savannah River Site

Aiken, SC 29808

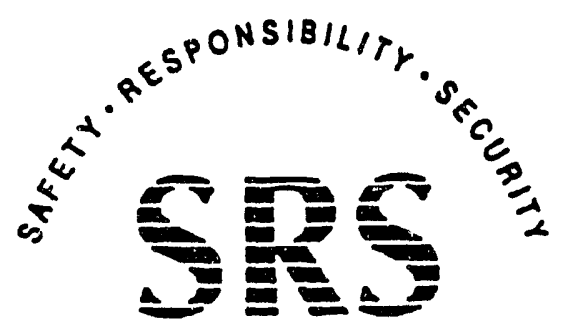


This page was left blank intentionally. 


\section{CONTENTS}

List of Figures $\ldots \ldots \ldots \ldots \ldots \ldots \ldots \ldots \ldots \ldots \ldots \ldots \ldots \ldots \ldots \ldots \ldots \ldots \ldots \ldots, \ldots \ldots$

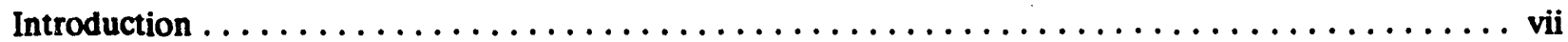

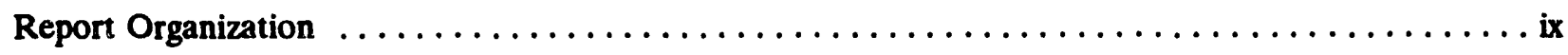

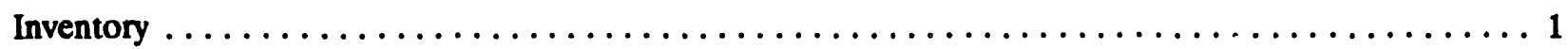

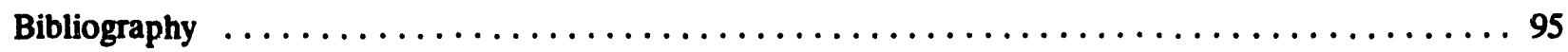

Building Number Index $\ldots \ldots \ldots \ldots \ldots \ldots \ldots \ldots \ldots \ldots \ldots \ldots \ldots \ldots \ldots \ldots \ldots \ldots$

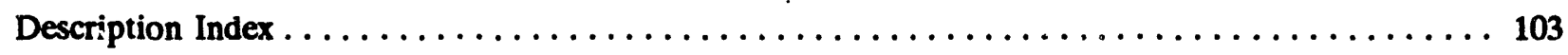

Description Index by Area $\ldots \ldots \ldots \ldots \ldots \ldots \ldots \ldots \ldots \ldots \ldots \ldots \ldots \ldots \ldots \ldots \ldots \ldots$

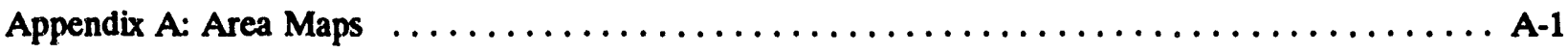


This page was left blank intentionally. 


\section{LIST OF FIGURES}

Figure A-1. Areas, Sites, the GBW Well, the FC 5 cluster, and PW Production Wells at the Savannah River Site A-2

Figure A-2. C Area, Separations Area, and Nearby Wells $\ldots \ldots \ldots \ldots \ldots \ldots \ldots \ldots \ldots$

Figure A-3. Groundwater Monitoring Wells In and Near C Area $\ldots \ldots \ldots \ldots \ldots \ldots \ldots \ldots$

Figure A-4. Groundwater Monitoring Wells In and Near K Area $\ldots \ldots \ldots \ldots \ldots \ldots \ldots \ldots$

Figure A-5. Groundwater Monitoring Wells at $\mathrm{L}$ Area $\ldots \ldots \ldots \ldots \ldots \ldots \ldots \ldots \ldots \ldots$

Figure A-6. Groundwater Monitoring Weils at P Area $\ldots \ldots \ldots \ldots \ldots \ldots \ldots \ldots \ldots \ldots$

Figure A-7. Groundwater Monitoring Wells at R Area $\ldots \ldots \ldots \ldots \ldots \ldots \ldots \ldots \ldots \ldots$

Figure A-8. Active Groundwater Monitoring Wells In and Near E Area and H-Area Seepage Basins $\ldots \ldots \ldots \ldots \ldots \ldots \ldots \ldots \ldots \ldots \ldots \ldots \ldots \ldots \ldots \ldots$

Figure A-9. Abandoned Groundwater Monitoring Wells In and Near E Area and

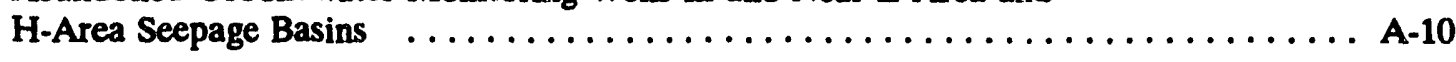

Figure A-10. Active Groundwater Monitoring Wells at the North Part of E Area $\ldots \ldots \ldots \ldots$ A-11

Figure A-11. Abandoned Groundwater Monitoring Wells at the North Part of E Area ........ A-12

Figure A-12. Groundwater Monitoring Wells Near F-Area Canyon Building and

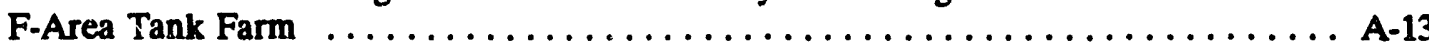

Figure A-13. Groundwater Monitoring Wells Near F-Area Seepage Basins and

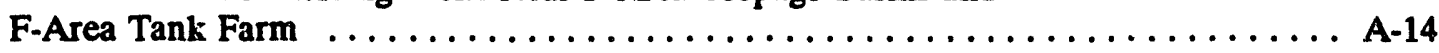

Figure A-14. Active Groundwater Monitoring Wells at the Central Part of H Area ......... A-15

Figure A-15. Abandoned Groundwater Monitoring Wells at the Central Part of H Area ...... A-16

Figure A-16. Groundwater Monitoring Wells at the North Part of H Area, at S Area, Y Area,

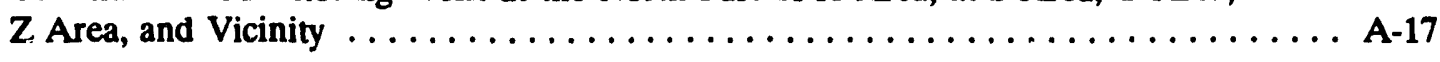

Figure A-17. Groundwater Monitoring Wells at A and M Areas and Silverton Road Waste Site .... A-18

Figure A-18. Active Groundwater Monitoring Wells at $\mathrm{A}$ and $\mathrm{M}$ Areas $\ldots \ldots \ldots \ldots \ldots$........

Figure A-19. Abandoned Groundwater Monitoring Wells at A and M Areas .......... A-20 
Figure A-20. Groundwater Monitoring Wells at D Area $\ldots \ldots \ldots \ldots \ldots \ldots \ldots \ldots \ldots \ldots$ A-21

Figure A-21. Groundwater Monitoring Wells at and Near the Central Shops Area and the

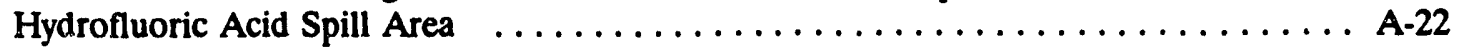

Figure A-22. Groundwater Monitoring Wells at the Chemicals, Metals, and Pesticides Pits $\ldots \ldots$ A-23

Figure A-23. Groundwater Monitoring Wells at Site B (Interim Waste Technology Site/Proposed

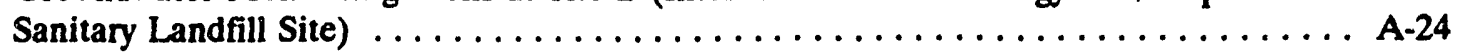

Figure A-24. Groundwater Monitoring Wells at Sites $P$ and $Q$ (Interim Waste Technology Sites), the New Sanitary Landfill, and the Kato Road and Lower Kato Road Sewage

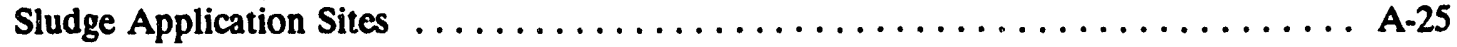

Figure A-25. Groundwater Monitoring Wells at Par Pond $\ldots \ldots \ldots \ldots \ldots \ldots \ldots \ldots \ldots \ldots$

Figure A-26. Groundwater Monitoring Wells at Road A Chemical Basin and 40-Acre Hardwood Sewage Sludge Application Site $\ldots \ldots \ldots \ldots \ldots \ldots \ldots \ldots \ldots$ A-27

Figure A-27. Groundwater Monitoring Wells at the Sanitary Landfill and the Orangeburg

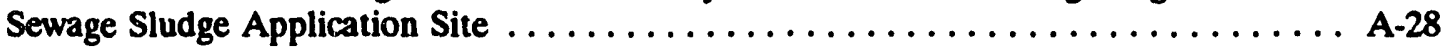

Figure A-28. Groundwater Monitoring Wells at Sandy (Lucy) and Road F Sewage Sludge Application Sites . . . . . . . . . . . . . . . . . . . . . . . . . A-29

Figure A-29. Groundwater Monitoring Wells at $\operatorname{TNX}$ Area $\ldots \ldots \ldots \ldots \ldots \ldots \ldots \ldots$ 


\section{INTRODUCTION}

This report is an inventory of the wells contained in Environmental Protection Department/Environmental Monitoring Section (EPD/EMS) documents since the startup of the Savannah River Site (SRS) and includes wells monitored by special request and SRS research wells. Wells listed in this inventory are monitoring wells unless otherwise indicated.

The purpose of this report is as follows:

- to provide a historical record of the wells that EPD/EMS has monitored

- to provide a document containing a list of wells that are currently in the EPD/EMS Groundwater Monitoring Program

- to provide pertinent information about all wells listed in EPD/EMS documents

The wells contained in this report are organized alphabetically, based on the Well Data File code. An " $R^{n}$ as the second letter in the well ID designates a replacement well with the same name as the original well. Both active and abandoned wells are included. Abandoned wells are noted with the abandonment date; wells with no abandonment date are assumed to be active. Wells under construction are not included. Missing or unknown information about a well is indicated by a blank space in the inventory.

The north and east coordinates, ground and casing (TOC) elevations for the wells are taken from surveying reports by Surveying and Mapping Consultants, Inc. of Greenville, South Carolina, if they have been surveyed. These data are taken from available secondary sources if the wells have not been surveyed.

Screen Zone Elevation values in parentheses are in feet below mean sea level (MSL). Elevations not in parentheses are above MSL.

The effective well depth is calculated by subtracting the bottom of the screen from the TOC.

The following abbreviations are used under casing type:

$$
\begin{aligned}
& \text { Al }=\text { aluminum } \\
& \text { CS }=\text { carbon steel } \\
& \text { PVC }=\text { polyvinyl chloride } \\
& \text { SS }=\text { stainless steel } \\
& \text { Steel }=\text { galvanized steel }
\end{aligned}
$$

** = These wells have a smaller casing within a larger casing. The outer casing does not extend as deeply as the inner casing. See individual series for further explanation.

As of the fourth quarter 1981, the policy for installing new wells is to use PVC casing. If no information to the contrary is available, casing type is listed as PVC for all wells installed during and after the fourth quarter of 1981 and as steel for all wells installed prior to that date. 


\section{Introduction}

If available, the last date of grouting is used as the installation date. This date does not necessarily indicate that a well is ready for sampling. The following symbols are used for approximate install and abandon dates in this report:

- An asterisk next to an installation date indicates the first day of the quarter in which the well was first sampled. An asterisk next to an abandon date indicates the first day of the quarter after the well was last sampled.

An approximate symbol indicates that the approximate date is taken from an annual or semiannual environmental report.

This report is a revision and update of Environmental Protection Department's Well Inventory, ESH-EMS910034. Because of new well installations and continuing research of historical documents, updates of this document are issued at approximately six-month intervals. If possible, information is confirmed by a verified hard copy in the Groundwater Records Library at SRS. However, in some cases, information is unknown or approximate (as noted). Later editions of this report will contain newly discovered information, resurveys of existing wells, new abandonments, and entries for all new wells. If you have additional information about the wells listed in this document, please forward such data to C. D. Rogers at Building 735-16A.

The following new well installations have been added to this edition of the Inventory:

BGO 8AR, 10DR, 40D, 45A, 45B, 45C, 45D

BGX 2B, 2D, 3D, 4A, 4C, 4D, 5D, 9D, 10D, 12C, 12D

BTP 1D, 1DD, 2D, 2DD, 3D, 3DD, 4D, 4DD, 5C, 6C, 6D, 6DD, 7C, 7D, 7DD, 8C, 8D, 8DD, 9C, 9D, 9DD, 10C, 10D, 10DD, 11C, 11D, 11DD, 12C, 12D, 12DD

HMD 1D, 2D, 3D, 4D

HXB 4D, 5D

IDB 2CC

LFW 43B, 43C, 43D, 44D, 45D, 46D, 47C, 47D, 48C, 48D, 55C, 55D, 56D, 57B, 57C, 57D, 58D, 59B, 59C, 59D, 60D, 61C, 61D, 62B, 62C, 62D

MVC 1A, 1B, 1C, 2A, 2B, 2C, 3A, 3B, 3C

MVE 1, 2, 3, 4 


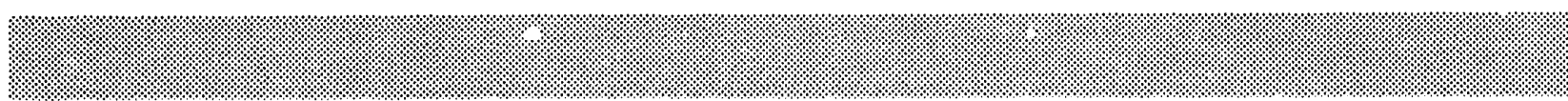

\section{REPORT ORGANIZATION}

This report organizes the wells alphabetically, based on the Well Data File code. Included under each well series group is the following information:

- descriptive location of the well series

- building number (if applicable)

- reference to an area map on which the well series is located (if applicable)

- well number (ID)

- SRS coordinates

- elevation (ft MSL) of the screen zone

- elevation (ft MSL) of the ground elevation

- elevation (ft MSL) of the elevation of the top of the casing (TOC)

- effective well depth (ft from TOC to bottom of screen)

- diameter and type of casing

- date of installation

- date of abandonment (if applicable)

- notes specific to the well series (if applicable)

Appendix A contains area maps that show the locations of the EPD/EMS wells. These maps were prepared in AutoCAD॰ by Exploration Resources of Athens, Georgia. Disk and hard copies are available from EPD/EMS. 
This page was left blank intentionally. 


\section{A WELL SERIES}

Description: Swamp area southeast of H-Area Se page Basins Bldg. Number: Not applicable

Map: Not applicable

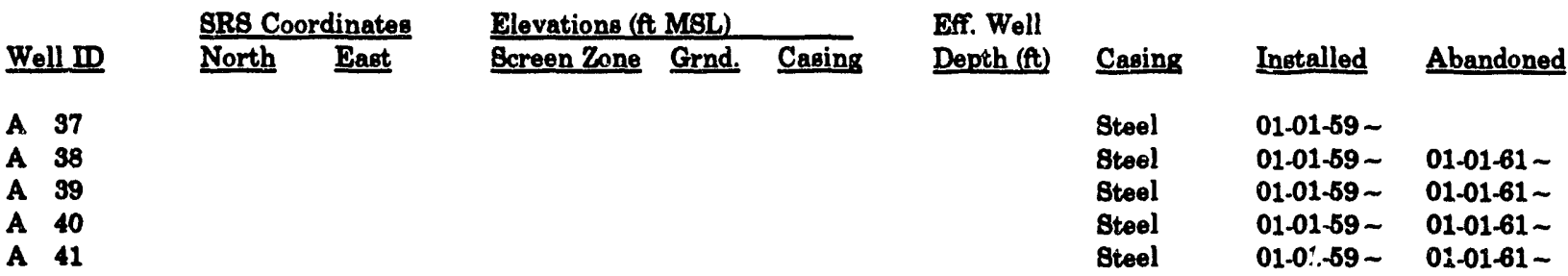

Notes: According to Works Technical Department (1962), A 38, 39, 40, and 41 were not monitored from January through June 1961. There is no evidence that these wells were monitored later. They were probably abandoned by early 1961.

\section{ABG WELL SERIES}

The ABG Series was renamed the ABW Series in September 1984 according to Table A-1, "Prefix Letter and Project Series in AM Area," Marine and Bledsoe (1985). See the ABW Series.

\section{ABP WELL SERIES}

Description: A-Area Metals Burning Pit

Bldg. Number: 731-4A

Map: Figure A-17

\begin{tabular}{lll} 
& \multicolumn{2}{l}{ SRS Coordinates } \\
\cline { 3 - 3 } Well ID & North & East \\
ABP 1 & 97531.7 & 44461.9 \\
ABP 1A & 97501.6 & 44425.6 \\
ABP 1DD & 97511.4 & 44439.6 \\
ABP 2 & 97752.2 & 44094.2 \\
ABP 2A & 97764.3 & 44118.8 \\
ABP 2DD & 97753.7 & 44126.7 \\
ABP 3 & 97794.1 & 44509.3 \\
ABP 3C & 97778.2 & 44506.3 \\
ABP 4 & 97489.7 & 44096.0 \\
ABP 4DD & 97495.5 & 44101.3 \\
ABP 6D & 97889.7 & 44101.4 \\
ABP 7D & 97449.7 & 43930.1 \\
ABP 8C & 97855.6 & 43968.7 \\
ABP 8D & 97854.9 & 43984.1
\end{tabular}

\begin{tabular}{|c|c|c|}
\hline \multicolumn{3}{|c|}{ Elevations (A MSL) } \\
\hline Screen Zone & Grnd. & Caging \\
\hline 238.4-208.1 & 358.3 & 360.4 \\
\hline 202.9-172.9 & 357.9 & 359.9 \\
\hline $227.2-207.2$ & 357.9 & 360.1 \\
\hline $244.9-214.9$ & 369.9 & 371.9 \\
\hline 211.1-181.1 & 370.1 & 371.9 \\
\hline 222.3-202.2 & 368.3 & 370.6 \\
\hline 236.9-206.9 & 361.9 & 953.7 \\
\hline $165.3-160.3$ & 352.3 & 354.6 \\
\hline 212.5-182.5 & 362.5 & 364.3 \\
\hline 223.2-203.2 & 362.7 & 365.0 \\
\hline 222.4-202.4 & 363.0 & 365.3 \\
\hline $225.2-205.2$ & 361.9 & 364.2 \\
\hline 190.6-185.5 & 369.8 & 372.1 \\
\hline 228.2.208.1 & 368.8 & 370.9 \\
\hline
\end{tabular}

Eff. Well

Depth (ft) Casing Installed Abandoned

$152.0 \quad 4^{\prime \prime}$ FVC $\quad 08-01-83 \quad 07-01-84^{*}$

$187.0 \quad 4^{*}$ PVC $08-15-84$

$152.9 \quad 2^{n}$ PVC $06-30-89$

$157.0 \quad 4^{n}$ PVC $08-01-89$

$190.8 \quad 4^{n}$ PVC 08-10-84

$168.4 \quad 2^{n}$ PVC 06-23-89

$146.8 \quad 4^{n}$ PVC $\quad 08-04-83$

$194.2 \quad$." PVC $07-10-89$

$181.8 \quad 4^{\prime \prime}$ PVC 06-21.84

$161.8 \quad 2^{n}$ PVC $\quad 06-23-89$

$162.9 \quad 2^{n}$ PVC 07.05-89

$159.0 \quad 2^{n}$ PVC $06-30-89$

$186.6 \quad 4^{n}$ PVC $\quad 07-20-89$

$\begin{array}{lll}162.8 & 4 \text { " PVC } & 07-20-89\end{array}$

Notes: ABP 1DD, 2DD, 4DD, 6D, and TD are piezometers. 


\section{ABW WELL SERIES}

Description: A-Area Background Well near Firing Range

Bldg. Number: 700-A-1

Map: Figure A-18

\begin{tabular}{|c|c|c|c|c|c|c|c|c|}
\hline & SRS Coordinates & Elovations (f & MSL) & & Eff. Well & & & \\
\hline Well ID & North East & Screen Zone & Grnd. & Casing & Depth (t) & Casing & Installed & Abandon \\
\hline ABW 1 & $105939.9 \quad 56016.4$ & 215.1-185.1 & 323.1 & 324.8 & 139.7 & $4^{n}$ PVC & $06-02-89$ & \\
\hline
\end{tabular}

Notes: The ABG Beries was renamed the ABW Beries in Beptember 1984 according to Table A-1, "Prefix Letter and Project Series in AM Area," Marine and Bledeoe (1985).

$A B W 1$ is often referred to as the $700-A$ well.

\section{AC WELL SERIES}

Description: A-Area Cluster Perimeter Wells and M-Area Plume Definition Wells Bldg. Number: Not applicable

Map: Figures A-17 and A-18

\begin{tabular}{|c|c|c|c|c|c|c|c|c|c|}
\hline \multirow[b]{2}{*}{ Well ID } & \multicolumn{2}{|c|}{ SRS Coordinates } & \multicolumn{3}{|c|}{ Flevations (ft MSL } & \multirow{2}{*}{$\begin{array}{l}\text { Dff. Well } \\
\text { Depth (At) }\end{array}$} & \multirow[b]{2}{*}{ Caring } & \multirow[b]{2}{*}{ Installed } & \multirow[b]{2}{*}{ Abandoned } \\
\hline & North & Fast & Ecreen Zone & Grnd. & ming & & & & \\
\hline IA & 106866.0 & 42238.8 & $146.7-140.7$ & 260.7 & 282.1 & 121.4 & $4^{\prime \prime}$ Stoel & $08-03-81$ & \\
\hline AC & 105882.8 & 42250.5 & 202.1-197.1 & 261.1 & 262.0 & 64.9 & Steol & $07-28-81$ & \\
\hline AC $2 A$ & 105638.4 & 46428.6 & $146.0-141.0$ & 342.7 & 944.7 & 208.7 & \& PVC $^{n}$ PV & $09-09-81$ & \\
\hline AC & 106648.7 & 46444.6 & $296.4-216.4$ & $\mathbf{3 4 2 . 8}$ & 344.8 & 128.4 & 4" PVC & $08-12-81$ & \\
\hline AC & 100989.1 & 42119.8 & $16 \$ .6-148.6$ & 900.4 & 302.3 & 158.7 & $4^{n}$ PVC & $09-16-81$ & \\
\hline $\mathbf{A C}$ & 100996.6 & 42113.6 & $213.4-193.4$ & 300.1 & 302.5 & 109.1 & $4^{n}$ PVC & $08-21-81$ & \\
\hline
\end{tabular}

\section{ACB WELL SERIES}

Description: A-Area Coal Pile Runoff Containment Basin

Bldg. Number: 788-3A

Map: Figures A-18 and A-19

\begin{tabular}{|c|c|c|c|c|c|c|c|c|c|}
\hline \multirow[b]{2}{*}{ Well ID } & \multicolumn{2}{|c|}{ SRS Coordinates } & \multicolumn{3}{|c|}{ Elevations (ft MSL) } & \multirow{2}{*}{$\begin{array}{l}\text { Eff. Well } \\
\text { Depth (ft) }\end{array}$} & \multirow[b]{2}{*}{ Casing } & \multirow[b]{2}{*}{ Installed } & \multirow[b]{2}{*}{ Abandoned } \\
\hline & North & East & Bcreen Zone & Grnd. & Casing & & & & \\
\hline $\begin{array}{ll}\text { ACB } 1 \\
A C B & 1 A\end{array}$ & $\begin{array}{l}102624.4 \\
102622.9\end{array}$ & $\begin{array}{l}51361.7 \\
51369.9\end{array}$ & $\begin{array}{l}270.9-250.9 \\
247.6-217.6\end{array}$ & $\begin{array}{l}357.5 \\
357.6\end{array}$ & $\begin{array}{l}359.6 \\
359.6\end{array}$ & $\begin{array}{l}108.6 \\
142.0\end{array}$ & $\begin{array}{l}4^{n} \text { Steel } \\
4^{n} \text { PVC }\end{array}$ & $\begin{array}{l}01-22-81 \\
01-11-84\end{array}$ & $02-03-84$ \\
\hline ACB 2 & 102349.2 & 51659.2 & 253.2-233.2 & 347.2 & 349.2 & 116.0 & 4" Steel & $03-24-80$ & $01.01 .84^{*}$ \\
\hline$A C B \quad 2 A$ & 102967.4 & 61661.3 & $237.8-207.8$ & $\mathbf{3 4 7 . 8}$ & $\$ 49.8$ & 142.0 & & $01-30-84$ & \\
\hline $\mathrm{ACB} 3$ & 102161.6 & 51331.9 & $262.0-242.0$ & 347.6 & 349.5 & 107.6 & $4^{n}$ Steel & $05-02-80$ & $01-01-84^{*}$ \\
\hline ACB SA & 102154.3 & 51313.3 & 236.3-206.3 & 346.3 & 348.3 & 142.0 & $4^{\prime \prime}$ PVC & 01.25 .84 & \\
\hline $\mathrm{ACB} 4$ & 102324.0 & 51114.4 & $250.7-230.7$ & 347.6 & 349.6 & 118.8 & $4^{\prime \prime}$ Btoel & $01-19-81$ & $01-01.84 *$ \\
\hline ACB $4 A$ & 102343.9 & 61116.2 & 241.7-211.7 & 356.7 & 369.1 & 147.4 & $4^{\prime \prime}$ PVC & $02-02-84$ & \\
\hline
\end{tabular}




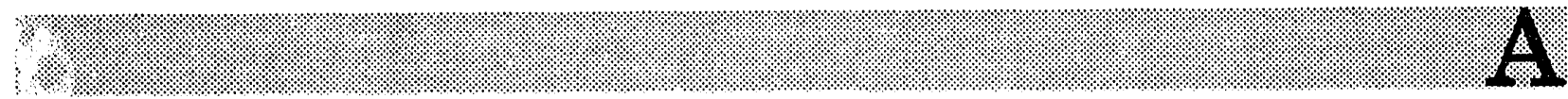

\section{AMB WELL SERIES}

Description: Metallurgical Laboratory Seepage Bas

Bldg. Number: 904-110G

Map: Figures A-18 and A-19

\begin{tabular}{|c|c|c|c|c|c|c|c|c|c|}
\hline \multirow[b]{2}{*}{ Well ID } & \multicolumn{2}{|c|}{ ERS Coordinates } & \multicolumn{3}{|c|}{ Dlevations (A MSL) } & \multirow{2}{*}{$\begin{array}{l}\text { Eff. Well } \\
\text { Depth (ft) }\end{array}$} & \multirow[b]{2}{*}{ Casing } & \multirow[b]{2}{*}{ Installed } & \multirow[b]{2}{*}{ Abandoned } \\
\hline & North & East & Bcreen Zone & Grnd. & Casing & & & & \\
\hline AMB 1 & 103993.5 & 51425.5 & $260.8-230.8$ & 375.8 & 377.8 & 147.0 & $4^{n}$ PVC & $06-07-83$ & $04-01-84 *$ \\
\hline AMB 1A & 104022.5 & 51436.3 & 246.9-216.9 & 376.9 & 378.7 & 161.8 & 4" PVC & $06-29-84$ & $08-22-88$ \\
\hline AMB 2 & 104164.6 & 61517.9 & $252.0-222.0$ & 377.0 & 379.3 & 167.3 & $4^{n}$ PVC & $06-13-83$ & $08-12-88$ \\
\hline AMB 3 & 104012.9 & 51603.5 & 266.8-236.8 & 371.8 & 373.8 & 137.0 & $4^{n}$ PVC & $05.29-83$ & $04-01-84 *$ \\
\hline AMB 3A & 104011.6 & 51625.1 & $251.4-221.4$ & 371.4 & 373.3 & 161.9 & 4" PVC & $04-11-84$ & $08-22-88$ \\
\hline AMB 4 & 104125.6 & 51460.4 & 242.8-222.8 & 378.6 & 380.4 & 157.6 & $4^{\prime \prime}$ PVC & $09-08-80$ & \\
\hline Aur 5 & 104083.4 & 51467.2 & 242.1-222.1 & 377.6 & 979.6 & 167.6 & $4^{n}$ PVC & $09-14-88$ & \\
\hline AMB 6 & 104034.1 & 61466.0 & $242.6-222.6$ & 375.1 & 377.2 & 164.6 & $4^{*}$ PVC & $09.16-88$ & \\
\hline$A M B$ & 103920.0 & 61624.9 & 242.1-222.1 & 368.1 & 369.9 & 147.8 & $4^{n}$ PVC & $09-06-88$ & \\
\hline AMIB 8 & 103730 & 51380 & & & & & 4" PVC & $10-17-89$ & $10-25-89$ \\
\hline AMB 8D & 103874.7 & 51400.5 & $240.8-220.8$ & 367.8 & 369.6 & 148.8 & $4^{n}$ PVC & $10-31-89$ & \\
\hline AMB 9 & 103465 & 51320 & & & & & $4^{n}$ PVC & $06-28-89$ & $10-31-89$ \\
\hline AMB 9D & 103585.2 & 51263.0 & 299.7-219.7 & 365.7 & 367.9 & 148.2 & 4" PVC & $10-25-89$ & \\
\hline AMB 10 & 103280 & 51455 & & & & & 4" PVC & $10-10-89$ & $11-03-89$ \\
\hline AMB 10D & 103293.4 & 51456.0 & $299.4-219.4$ & 363.4 & 965.5 & 146.1 & $4^{n}$ PVC & $11-03-89$ & \\
\hline AMB 10DD & 108278.7 & 51466.0 & $358.6-338.6$ & 363.6 & 336.4 & 26.8 & 4" PVC & 06-07-89 & \\
\hline AMB 11D & 103132.3 & 51992.6 & $240.6-220.5$ & 362.0 & 364.0 & 143.5 & $4^{n}$ PVC & $06-13-89$ & \\
\hline AMB 12D & 103602.4 & 51901.6 & $239.4-219.4$ & 367.8 & 369.8 & 150.4 & $4^{n}$ PVC & 08-16-89 & \\
\hline
\end{tabular}

1. The 8BA Beries was renamed the AMB Berien in Auguet 1984 according to Table A-1, "Prefix Letter and Project Beries in AM Area," Marine and Blodeoe (1385).

According to page 1 of the Daily Activitien Report for AMB 9 dated 10-23-89, a development crew diecovered that the casing for ANB 8 had collapeed. This hole was abandoned 10-25-89. AMB 8D was inotalled 10-91-89 to replace the collapeed well.

According to page 1 of the Daily Activitien Report for ANB 8 and 8 dated 10-17.89, the PVC casing for AMB 9 malted after grouting. This hole was abandoned 10-25-89. AMB 9D was installed 10-25-89 to replace AMAB 9.

According to Daily Activity Reporte for AMB 10, page 1 dated 10-09-89 and page 2 datod 10-31-89, grout contaminatod the filter pack. This hole was abandoned 11-03-89. IAB 10D was inotalled 11-08-89 to replace AMB 10.

\section{AOB WELL SERIES}

Description: Motor Shop Oil Basin

Bldg. Number: 904-101G

Map: Figure A-18

\begin{tabular}{|c|c|c|c|c|c|c|c|c|}
\hline & Bns Coordinates & Elevations ( & MSL) & & Bff. Well & & & \\
\hline Well ID & North Eagt & Bcreen Zone & Grnd. & Casing & Depth (ft) & Cenging & Installed & Abandoned \\
\hline IOB 1 & 101910.7 & 248.5.218.5 & 338.5 & 341.1 & 122.6 & 4" PVC & $06-27-83$ & \\
\hline
\end{tabular}

(sories continued) 
AOB WELL SERIES (cont.)

\begin{tabular}{|c|c|c|c|c|c|c|c|c|c|}
\hline & SR8 Coor & dinates & Elovations ( $f$ & MSL) & & Eff. Well & & & \\
\hline Well D & North & Fagt & Screen Zone & Grind. & Casing & Depth (tt) & Casing & Installed & Abandoned \\
\hline $\begin{array}{l}A O B 2 \\
A O B \\
A\end{array}$ & $\begin{array}{l}102009.8 \\
102164.4\end{array}$ & $\begin{array}{l}60724.7 \\
60959.4\end{array}$ & $\begin{array}{l}250.2-220.2 \\
243.9-223.9\end{array}$ & $\begin{array}{l}343.2 \\
350.6\end{array}$ & $\begin{array}{l}346.4 \\
352.6\end{array}$ & $\begin{array}{l}125.2 \\
128.7\end{array}$ & $\begin{array}{l}4^{\prime \prime} \text { PVC } \\
4^{\prime \prime} \text { PVC }\end{array}$ & $\begin{array}{l}05-27-83 \\
04-21-89\end{array}$ & \\
\hline
\end{tabular}

Notes: The AOP Series was renamed the AOB Series in August 1984 according to Table A-1, "Prefix Letter and Project Series in AM Area," Marine and Bledeoe (1985).

\section{AOP WELL SERIES}

The AOP Series was renamed the AOB Series in August 1984 according to Table A-1, "Prefix Letter and Project Series in AM Area," Marine and Bledsoe (1985). See the AOB Series.

\section{ARP WELL SERIES}

Description: A-Area Burning/Rubble Pits

Bldg. Number: 731-A, -1A

Map: Figure A-17

\begin{tabular}{|c|c|c|c|c|c|c|c|c|c|}
\hline \multirow[b]{2}{*}{ Well ID } & \multicolumn{2}{|c|}{ 8R8 Coordinates } & \multicolumn{3}{|c|}{ Hevations (A MSL) } & \multirow{2}{*}{$\begin{array}{l}\text { Defi. Well } \\
\text { Depth (A) }\end{array}$} & \multirow[b]{2}{*}{ Casing } & \multirow[b]{2}{*}{ Inctalled } & \multirow[b]{2}{*}{ Abandoned } \\
\hline & North & Eagt & Scroen Zone & Grad. & Caping & & & & \\
\hline ARP 1A & 99102.9 & 44917.4 & $223.0-198.0$ & 363.0 & 365.1 & 162.1 & 4* PVC & $02-03-84$ & \\
\hline ARP 2 & 99119.8 & 44876.1 & $220.3-190.3$ & 336.3 & 337.3 & 147.0 & 4" PVC & $12-22-83$ & \\
\hline ARP 3 & 98698.2 & 44903.7 & 218.2-188.2 & 398.2 & 399.8 & 161.6 & t"I & $12.14-83$ & \\
\hline ARP 4 & $\$ 18687.7$ & 44974.8 & 227.8-197.8 & 346.8 & $\mathbf{3 4 8 . 4}$ & $\therefore 50.6$ & 4" PVC & $06-26-84$ & \\
\hline
\end{tabular}

Nstas: According to the Field Activity Log for ARP 1, the well was inotallod 12-16-83 and developed for approximatoly 2.5 dayn, but the well would not produce. Conenquently, the well was abandoned.

According to the Field Activity Log for ARF 3, the well was firot drilled 12-05-83, redrilled 12-08-83, drilled a third time on 12-14-83 and finally inotallod. 
ASB WELI SERIES

Description: Savannah River Laboratory (SRL) Seepage Basins

Bldg. Number: 904-53G1, -53G2, -54G, -55G

Map: Figure A-18

\begin{tabular}{|c|c|c|c|c|c|c|c|c|c|}
\hline & BRS Coor & dinates & Plevations ( $\mathrm{f}$ & MBL) & & EAf. Woll & & & \\
\hline Well ID & North & Eant & Screen Zone & Grnd. & Casing & Depth (ft) & Caxing & Installed & Abandoned \\
\hline ASB 1 & 105530.8 & 52621.8 & 257.6-237.6 & 346.7 & 348.7 & 111.1 & 4" Bteel & $08-12-81$ & $01-05-84$ \\
\hline A8B 1A & 105536.0 & 52614.0 & 247.2-217.2 & 347.2 & 349.1 & 131.9 & 4" PVC & $01-05-84$ & \\
\hline ASB 2 & 105608.3 & 52846.6 & 255.9-235.9 & 346.5 & 348.6 & 112.6 & $4^{n}$ Stoel & $03-16-81$ & $01-06-84$ \\
\hline ABB 2A & 105608.8 & 52856.9 & 246.9.216.9 & 346.9 & 849.0 & 132.1 & 4" PVC & $12.30-83$ & \\
\hline ABB 2C & 105605.4 & 62843.6 & $177.4-172.4$ & 347.4 & 349.4 & 177.0 & 4" PVC & $11-30-90$ & \\
\hline ASB 3 & 106660.6 & 63163.7 & & 343.2 & 345.2 & & 4" Bteel & $03-20-81$ & $10-01-81$ \\
\hline ASB 3A & 105657.4 & 63162.7 & 247.9-217.9 & 342.9 & 346.0 & 127.1 & 4" PVC & $10-13-81$ & \\
\hline AsD 3C & 105668.8 & 53160.9 & 173.8.168.8 & 349.8 & 346.0 & 177.2 & 4" PVC & $11-29-90$ & \\
\hline ASB 4 & 105935.7 & 69177.2 & 256.1-226.1 & 333.1 & 395.6 & 109.6 & 4" PVC & $08-20-81$ & \\
\hline AsB 5 & 105891.8 & 52876.3 & 242.4-222.4 & 342.8 & 344.8 & 122.4 & Bteel & $03-05-81$ & $02-14-84$ \\
\hline ASB SA & 105885.5 & 52865.7 & 247.9-217.9 & 342.9 & $\mathbf{3 4 5 . 0}$ & 127.1 & $4^{N}$ PVC & $02.13-84$ & \\
\hline A8B 6 & 105721.1 & 62683.1 & 249.9-223.9 & 348.4 & 360.4 & 126.6 & 4" Bteol & 03-03-81 & $02-20-84$ \\
\hline ABB 6A & 106716.0 & 62676.9 & 248.2-218.2 & 348.2 & 360.2 & 132.0 & 4" PVC & $02-20-84$ & \\
\hline ABB 6AA & 105727.0 & 62648.9 & 82.8- 78.1 & 951.8 & 364.2 & 276.1 & 4" PVC & $12-09-88$ & \\
\hline ABB 6C & 106739.8 & 62666.9 & $178.6-173.8$ & 351.6 & 363.6 & 179.8 & 4" PVC & $12.21-80$ & \\
\hline ABB GTA & 106749 ? & 62671.3 & 40.0-34.6 & 361.0 & 362.9 & 318.4 & $*$ & $01-30-91$ & \\
\hline ASB 7 & 105770.6 & 52626.4 & 231.3-211.3 & 351.3 & 353.4 & 142.1 & PVC & & \\
\hline A8B 8 & 106381.6 & $631 \div 3.6$ & 226.6-206.6 & 346.6 & 3480 & 142.4 & PVC & & \\
\hline ABB $8 A$ & 106569.3 & 63117.5 & 83.6. 77.9 & 347.2 & 349.3 & 271.4 & $4^{\circ}$ PVC & $08-26-85^{\wedge}$ & \\
\hline ABB $8 B$ & 108362.3 & 68109.6 & $128.4-122.8$ & 347.6 & 349.8 & 227.0 & 4" PVC & $12-14-84^{\wedge}$ & \\
\hline A8B $8 C$ & 106554.4 & 59101.0 & 188.3-182.7 & 347.6 & 349.7 & 167.0 & $4^{\prime \prime}$ PVC & $12.18-84^{\wedge}$ & \\
\hline ASB $8 T A$ & 106375.8 & 69124.7 & 24.6- 19.4 & 347.3 & 349.6 & 390.2 & $4^{\prime \prime} \mathrm{Cs}$ & $10-17-84^{\wedge}$ & \\
\hline A8B 9 & 104589.2 & 64228.2 & $236.4-216.4$ & 306.4 & 909.0 & 92.6 & PVC & & \\
\hline ASB 9B & 104664.7 & 54216.3 & $164.4-168.8$ & 306.6 & 309.0 & 160.2 & $4^{n}$ PVC & $07-16-87$ & \\
\hline ABB $9 C$ & 104668.1 & 54201.1 & 182.9-178.2 & 307.9 & 309.9 & 191.7 & 4" PVC & $02-16-89$ & \\
\hline $\mathrm{ABB} 10 \mathrm{C}$ & 105673.1 & 52905.5 & $176.8-171.8$ & 946.8 & $\$ 48.9$ & 177.1 & $4^{n}$ PVC & $11-26-90$ & \\
\hline
\end{tabular}

Notes: ABB aA was originally drilled on 2-03-34. According to the Well Conotruction Dotails dated 2-09-84, the screen was groutod during finishing, and the well was redrillod. On 2-20-84 the well was comploted.

^The inotalled dates for theee wells are the dates on the caeing tally sheete in Goraghty \& Millor (1986).

** ABB GTA has 4-in. PVC casing inside 12-in. C8 caning. The wreen is atainlese etoel.

\section{ASP Wells}

Description: Unofficial wells in Z Area

Bldg. Number: Not applicable

Map: Figure A-16

\begin{tabular}{|c|c|c|c|c|c|c|c|c|c|}
\hline \multirow[b]{2}{*}{ Well ID } & \multicolumn{2}{|c|}{ BRS Coordinates } & \multicolumn{3}{|c|}{ Elevations MSL } & \multirow{2}{*}{$\begin{array}{l}\text { Efr. Well } \\
\text { Depth (t) }\end{array}$} & \multirow[b]{2}{*}{ Caring } & \multirow[b]{2}{*}{ Ingtalled } & \multirow[b]{2}{*}{ Abandoned } \\
\hline & North & East & Bcreen Zone & Grnd. & Caning & & & & \\
\hline $\begin{array}{ll}\text { ASP } & 1 \\
\text { ASP } & 2\end{array}$ & $\begin{array}{l}75670 \\
76050\end{array}$ & $\begin{array}{l}66475 \\
66360\end{array}$ & & & & & & & $\begin{array}{l}02-26-87 \\
03-03-87\end{array}$ \\
\hline
\end{tabular}

(series continued) 
ASP WELLS (cont.)

\begin{tabular}{|c|c|c|c|c|c|c|c|c|}
\hline \multirow[b]{2}{*}{ Well ID } & \multicolumn{2}{|c|}{ SRS Coorciinates } & \multicolumn{2}{|l|}{ Elevations (ft MSL) } & \multirow{2}{*}{$\begin{array}{l}\text { Eff. Well } \\
\text { Depth (ft) }\end{array}$} & \multirow[b]{2}{*}{ Casing } & \multirow[b]{2}{*}{ Installed } & \multirow[b]{2}{*}{ Abandoned } \\
\hline & North & East & Screen Zone Grnd. & Casing & & & & \\
\hline ABP 3 & 76120 & 68590 & & & & & & $03-03-87$ \\
\hline A8P 4 & 75740 & 66700 & & & & & & $02-25-87$ \\
\hline A8P 6 & 75890 & 66540 & & & & & & $02-26-87$ \\
\hline
\end{tabular}

Notes: According to the Well Abandonment Detaild, these wells did not have screens or casinge when they were abandoned. The identification of these welle comes $\mathrm{sem}$ the abandonment sheets. The ABP designation is unofficial.

\section{BG WELL SERIES}

Description: Burial Grounds

Bldg. Number: See note below

Map: Figures A-8, A-9, A-11, A-12, and A-13

\begin{tabular}{|c|c|c|c|c|c|c|c|c|c|c|}
\hline \multirow{2}{*}{\multicolumn{2}{|c|}{ Well ID }} & \multicolumn{2}{|c|}{ BRs Coordinates } & \multicolumn{3}{|c|}{ Plevations (ft MSL) } & \multirow{2}{*}{$\begin{array}{l}\text { Eff. "Noll } \\
\text { Depth (t) }\end{array}$} & \multirow[b]{2}{*}{ Casing } & \multirow[b]{2}{*}{ Installed } & \multirow[b]{2}{*}{ Abandoned } \\
\hline & & North & Enet & Soreen 7one & Gind. & Caring & & & & \\
\hline BG & 1 & 74485 & 55650 & $224.5-214.5$ & 277.5 & 278.1 & 63.6 & 9" Steel & $0.21-66$ & $07-01-76 *$ \\
\hline BG & 2 & 74100 & 56290 & 226.1-216.1 & 271.9 & 272.5 & 66.4 & 9" Steel & $03-29-66$ & $07-01-76^{*}$ \\
\hline BG & $\mathbf{3}$ & 73865 & 56680 & 227.0-217.0 & 267.2 & 267.6 & 60.6 & 9" Btoel & $08-28-66$ & $07-01.76 *$ \\
\hline BG & 4 & 73840.1 & 66720.2 & 267.1-257.1 & 269.1 & 269.1 & 12.0 & 3" Steol & $04-18-68$ & $07-01-76 *$ \\
\hline BG & 6 & 74119.6 & 66960.2 & 223.2-213.2 & 275.8 & 276.2 & 63.0 & 9" Stoel & $08-26-66$ & $0401-73^{*}$ \\
\hline BG & 6 & 74768.5 & 66906.0 & 228.7-218.7 & 280.7 & 281.3 & 62.6 & S" Bteel & $0404-66$ & $07-01-76^{*}$ \\
\hline BG & 7 & 76276 & 66265 & 225.8-216.8 & 288.8 & 287.4 & 71.6 & S" Btoel & $04-18-66$ & $07-01-76^{*}$ \\
\hline BG & 8 & 74917.1 & 56765.6 & $230.4-220.4$ & 286.4 & 289.3 & 68.9 & 3" Steol & $04-06-66$ & $07-01-16^{*}$ \\
\hline BG & 9 & 74622.8 & 66246.1 & 229.5-119.6 & 282.5 & 282.9 & 163.4 & $3^{n}$ Btoel & $04-11-66$ & 07-01-76* \\
\hline BG & 10 & 73056.6 & 56108.8 & & 254.4 & 256.4 & & Btoel & $12-01-57$ & \\
\hline BG & $10 A$ & 73050 & 66140 & & 254.8 & 256.8 & & 3.5" Al & 06.1861 & $04-21-89$ \\
\hline BG & 10.7 & 79053 & 56098 & $113.7-111.7$ & 253.7 & 264.8 & 149.1 & $1^{*}$ Streel & $11-05-65$ & 04-03-89 \\
\hline BG & $10 C$ & 73060 & 66092 & $142.4-140.4$ & 262.4 & 254.3 & 113.9 & 1" Btoel & $08-29-68$ & $08-30-89$ \\
\hline BG & 11 & 79677 & 66078 & & 254.0 & 258.8 & & Steel & $05.18-61$ & $07-08-61$ \\
\hline BG & 11 & 79854 & 66179 & & 259.2 & 261.2 & & 8teel & $07.14-61$ & \\
\hline BG & 12 & 74790 & 54640 & & 267.7 & 269.7 & & $\mathbf{3}^{n} \mathrm{Al}$ & $08-18-61$ & $07-01-76^{*}$ \\
\hline BG & 13 & 77010 & 54998 & & 318.2 & 318.9 & & Btoel & $08-01-61$ & \\
\hline BG & 14 & 77800 & 65000 & & 324.1 & 328.3 & & Bteel & $05-26-61$ & \\
\hline BG & 16 & 76476 & 55978 & & 806.0 & 807.0 & & Bteel & $05-26-61$ & \\
\hline BG & 16 & 73085 & 57260 & & 284.0 & 286.1 & & Bteel & $07.03-61$ & \\
\hline BG & 17 & 75877 & 64675 & & 276.6 & 278.6 & & Bteel & $07-03-61$ & \\
\hline BG & 18 & 75668 & 54198 & & 262.3 & 264.7 & & Al & $07-03-61$ & $07.01-76^{*}$ \\
\hline BG & 19 & 73423 & 67979 & & 297.2 & $\$ 00.3$ & & Steel & $07-05-61$ & \\
\hline BG & 20 & 73794 & 66942 & & 265.3 & 267.6 & & Btoel & $07-14-61$ & \\
\hline BG & 21 & 74030 & 54120 & & 229.2 & 230.9 & & Btoel & $07.14-61$ & \\
\hline BG & 22 & 75927 & 63073 & & 280.8 & 283.4 & & Stoel & $07.14-61$ & \\
\hline BG & 23 & & & & & & & Bteel & $10-01-62 *$ & $10-01-70^{*}$ \\
\hline BG & 24 & & & & & & & $3^{n} \mathrm{Al}$ & $08-23-62$ & $07-01-76^{*}$ \\
\hline BG & 25 & & & & & & & $3^{n} \mathrm{Al}$ & $10-01-62 *$ & $07-01-76^{*}$ \\
\hline
\end{tabular}

Notes: BG 10 is used as the background well for the H-Area Beepage Banins.

(eeries continued) 


\section{BG WELL SERIES (cont.)}

BG 10 and the BG 10 cluster are aseociated with Bldg. Number 904-56G.

The Boring $\log$ for BG 11 says that the casing was pulled on 7.09-61 because of perched water. Eight uncased wells surrounding BG 11-BG 11A, 11B, 11C, 11D, 11F, 11F, 11G, and 11E-were drilled to define the extent of perched water around "old" BG 11. The Boring Loge for the BG 11 wells refer to the well with the coordinates of N 73677 and E 55076 as the "old" BG 11. The well with the coordinates of N 73854 and E 56179 is referred to as the "new" BG 11.

According to Boring Loge for the welle, three uncased welle were drilled near BG 16. They are referred to on the Boring Log9 as BG 16 8.W, BG 16 N.W., and BG $168 . \mathrm{E}$.

\section{BG WELL SERIES (cont.)}

Description: Just outside the fence surrounding the Burial Grounds; BG 43, 52, and 67 are inside the fence

Bldg. Number: 643-E, -7E, -28E

Map: Figures A-8, A-9, A-10, A-11, and A-12

\begin{tabular}{|c|c|c|c|c|c|c|c|c|c|c|}
\hline \multirow{2}{*}{\multicolumn{2}{|c|}{ Well ID }} & \multicolumn{2}{|c|}{ SRs Coordinates } & \multicolumn{3}{|c|}{ Flevations (f MSL) } & \multirow{2}{*}{$\begin{array}{l}\text { Dff. V oll } \\
\text { Depth (ft) }\end{array}$} & \multirow[b]{2}{*}{ Casing } & \multirow[b]{2}{*}{ Inotalled } & \multirow[b]{2}{*}{ Abendoned } \\
\hline & & North & Epat & Screen 7one & Grnd. & Casing & & & & \\
\hline BG & 26 & 79968.4 & 58809.7 & $230.7-210.7$ & 293.3 & 295.7 & 85.0 & $4^{n}$ Steel & $06-12-76$ & $04-14-88$ \\
\hline BG & 27 & 74368.7 & 58810.0 & $254.4-234.4$ & 295.4 & 297.4 & 63.0 & $4^{n}$ Stoel & 05.31 .76 & $04-16-88$ \\
\hline BG & 28 & 74762.0 & 88810.2 & $259.7-239.7$ & 298.7 & 295.7 & 56.0 & $4^{n}$ Steel & 05.14 .76 & $04-18-88$ \\
\hline BG & 29 & 75151.6 & 58809.9 & 251.6291 .6 & 292.6 & 294.6 & 63.0 & $4^{n}$ Btoel & $08-17-76$ & $04-18-88$ \\
\hline BG & 30 & 75650.1 & 58809.1 & 251.7-231.7 & 286.9 & 288.7 & 57.0 & $4^{n}$ Steel & $05.18-76$ & $04-19-88$ \\
\hline$B G$ & 31 & 75949.9 & 58808.7 & $243.3-229.3$ & 291.4 & 293.3 & 70.0 & 4" Streel & $05-18-76$ & $04-19-88$ \\
\hline BG & 2 & 76949.9 & 68809.6 & 246.9-228.9 & 294.9 & 296.9 & 70.0 & $4^{\prime \prime}$ Bteel & $05-19-76$ & $04-11-88$ \\
\hline BG : & 13 & 76479.9 & 58526.0 & $241.2-221.2$ & 29.3 & 291.8 & 70.0 & $4^{n}$ Bteel & $05-20-76$ & $04-1488$ \\
\hline BG & 34 & 76498.6 & 68107.4 & $237.4-217.4$ & $\ln 2$ & 282.4 & 65.0 & $4^{\prime \prime}$ Bteel & $05.20-76$ & $0412-88$ \\
\hline BG : & 35 & 76495.3 & 57726.4 & $248.0-228.0$ & $\operatorname{css} 3$ & 283.0 & 65.0 & $4^{n}$ Btoel & $05-21-76$ & $04-13-88$ \\
\hline BG & 36 & 76747.6 & 67620.3 & 243.3-223.3 & 279.2 & 281.3 & 68.0 & 4" Btoel & $05-21.76$ & $04-13-88$ \\
\hline BG & 37 & 78804.9 & 67251.0 & $247.8-227.8$ & 291.1 & 292.8 & 65.0 & $4^{N}$ Steel & $05-24-76$ & $04-26-88$ \\
\hline BG & 38 & 76806.0 & 66851.1 & 245.9-225.9 & 900.3 & 31)1.9 & 76.0 & 4" Stoel & $05-24-76$ & $04-26-88$ \\
\hline BG & 39 & 76804.9 & 66461.3 & 248.0 .226 .0 & $\$ 07.0$ & $9,99.0$ & 83.0 & $4^{n}$ Btool & $08-25-76$ & $04-22-88$ \\
\hline BG & 40 & 76805.1 & 66051.0 & $241.9-<21.9$ & 313.1 & 314.9 & 99.0 & $4^{n}$ Btoel & $05-26-76$ & $04-21-88$ \\
\hline BG & 41 & 76576.3 & 65868.8 & $241.0-221.0$ & $\$ 04.0$ & 306.0 & 86.0 & $4^{\prime \prime}$ Steel & $05-27-76$ & $04-21-88$ \\
\hline$B G$ & 42 & 76178.8 & 56869.5 & 297.1-217.1 & 298.3 & 300.1 & 83.0 & 4" Stoel & $06-26-76$ & $04-20-88$ \\
\hline BG & 43 & 75852.6 & 66059.4 & 242.9-222.9 & 302.1 & 308.9 & 81.0 & 4" Steel & $05-27-76$ & $05.16-88$ \\
\hline BG & 61 & 73864.3 & 58699.3 & 241.2-221.2 & 290.6 & 292.2 & 71.0 & 4" Bteel & 0Rn1-76 & $03-30-88$ \\
\hline BG & 52 & 75910.4 & 55524.3 & $243.8-223.8$ & 287.6 & 289.8 & 66.0 & 4" Steel & $07-06-76$ & \\
\hline BG & 69 & 76157.3 & 65079.9 & 234.7-214.7 & 283.8 & 285.7 & 71.0 & $4^{n}$ Steol & 07-07-76 & \\
\hline BG & 54 & 75837.9 & 54830.3 & 235.2-215.2 & 275.3 & 277.2 & 62.0 & $4^{n}$ Stoel & $07-07.76$ & \\
\hline RG & 55 & 75526.3 & 64590.6 & $234.9-214.9$ & 274.8 & 276.9 & 62.0 & $4^{n}$ Stoel & $07-08-76$ & \\
\hline BG & 56 & 75206.5 & 54481.9 & 230.9-210.9 & 272.6 & 274.9 & 64.0 & 4" Btoel & $07-20-76$ & \\
\hline BG & 67 & 75000.4 & 54820.0 & $234.6-214.6$ & 270.9 & 272.6 & 58.0 & $4^{\prime \prime}$ Bteel & 07-19-76 & \\
\hline BG & 68 & 74790.9 & 56162.3 & 238.2-218.2 & 276.1 & 278.2 & 60.0 & $4^{\prime \prime}$ Btoel & $07-16-76$ & \\
\hline BG & 59 & 74693.4 & 55508.3 & 237.7-217.7 & 280.9 & 282.7 & 65.0 & $4^{n}$ Steel & 07-09-76 & \\
\hline BG & 60 & 74386.3 & 65850.3 & 235.5-215.5 & 273.6 & 275.6 & 60.0 & $4^{n}$ Bteol & $07-09.76$ & \\
\hline BG & 61 & 74075.4 & 56360.8 & $245.0-225.0$ & 272.5 & 275.0 & 50.0 & 4" Steel & $07-12-76$ & \\
\hline BG & 62 & 73971.6 & 56530.9 & 242.5-222.5 & 270.5 & 272.5 & 50.0 & $4^{n}$ Steel & $07.12-76$ & \\
\hline BG & 63 & 73754.5 & 56870.5 & 244.2-224.2 & 272.4 & 274.2 & 50.0 & $4^{\prime \prime}$ Btoel & $07-13-76$ & \\
\hline BG & 64 & 73547.2 & 57212.4 & 247.3-227.3 & 283.3 & 285.3 & 58.0 & 4" Steel & $07-13-76$ & \\
\hline BG & 65 & 73340.6 & 57562.7 & 250.9-230.9 & 289.1 & 290.9 & 60.0 & 4" Bteel & $07-14-76$ & \\
\hline BG & 66 & 73585.0 & 57805.0 & $251.0-231.0$ & 294.3 & 296.0 & 65.0 & $4^{n}$ Steel & $07-14-76$ & \\
\hline BG 6 & 67 & 73954.1 & 57902.6 & 244.7-224.7 & 292.8 & 294.7 & 70.0 & $4^{n}$ Steel & $07-16-76$ & \\
\hline
\end{tabular}


BG WELL SERIES (cont.)

Description: Just north of the Burial Grounds

Bldg. Number: 643-7E, -28E

Map: Figures A-2, A-8, A-8, A-10, A-11, and A-14

\begin{tabular}{|c|c|c|c|c|c|c|c|c|c|}
\hline \multirow[b]{2}{*}{ Well ID } & \multicolumn{2}{|c|}{ gRs Coordinatus } & \multicolumn{3}{|c|}{ Flevations (A MBL) } & \multirow{2}{*}{$\begin{array}{l}\text { Eff. Well } \\
\text { Depth (tt) }\end{array}$} & \multirow[b]{2}{*}{ Casing } & \multirow[b]{2}{*}{ Installed } & \multirow[b]{2}{*}{ Abandoned } \\
\hline & North & Eagt & Screen Zone & Grnd. & Casing & & & & \\
\hline BG 88 & 76553.6 & 58251.6 & & 282.5 & $28 \% .2$ & & $4^{n}$ Stoel & $04-01-79 \sim$ & $09-17.88$ \\
\hline BG 69 & 76553.8 & 68226.2 & & 282.2 & 2134.3 & & $4^{n}$ Stoel & $04-01.79 \sim$ & $04-07-88$ \\
\hline BG 70 & 76574.2 & 58199.6 & & 285.3 & 286.9 & & $4^{n}$ Steel & 04-01-79 & $03-18-88$ \\
\hline BG 71 & 76571.1 & 68.249 .2 & & 286.3 & 2887.9 & & $4^{n}$ Btgel & 04-01-79 & $09-17.88$ \\
\hline BG 72 & 76602.5 & 682279 & & 285.2 & 287.3 & & $4^{n}$ Steel & $04.01 .79 \sim$ & $03-24-88$ \\
\hline BG 73 & 76619.7 & 68245.3 & & 282.6 & 284.6 & & $4^{*}$ Steel & 04-01-79 & $03-23-88$ \\
\hline BC. 74 & 76630.4 & 68223.5 & & 281.7 & 284.1 & & $4^{\prime \prime}$ Stoel & $04-01.79 \sim$ & $03-22-88$ \\
\hline BG 75 & 76641.9 & 58182.7 & & 280.8 & 282.8 & & $4^{\prime \prime}$ Btoel & $04-01-79 \sim$ & $03-22-88$ \\
\hline BG 76 & 76751.9 & 58394.4 & & 284.6 & 286.7 & & $4^{n}$ Stoel & $05-01-79 \sim$ & $12-09.87$ \\
\hline BG 77 & 76771.8 & 58279.1 & & 284.0 & 285.9 & & $4^{\prime \prime}$ Steel & $05.01-79 \sim$ & $12-09-87$ \\
\hline BG 78 & 76805.9 & 68277.0 & & 284.7 & 287.1 & & $4^{*}$ Steel & $05-01-79 \sim$ & $12-08-87$ \\
\hline BG 79 & 76800.6 & 58324.9 & & 284.8 & 286.6 & & $4^{\infty}$ Bteel & $05-01-79 \sim$ & 12-10-87 \\
\hline BG 80 & 76596.6 & 57962.6 & & 276.5 & 278.5 & & $4^{n}$ Steel & $05-01-79 \sim$ & $03-11.88$ \\
\hline BG 81 & 76621.9 & 67983.0 & & 277.0 & 279.4 & & $4^{n}$ Steel & $05-01-79 \sim$ & $09-14-88$ \\
\hline BG 82 & 76645.7 & 57957.7 & & 276.3 & 278.1 & & $4^{n}$ Steel & $05.01 .79 \sim$ & $02-15-88$ \\
\hline BG 83 & 76671.6 & 67984.5 & & 276.0 & 277.7 & & $4^{n}$ Bteel & 06-01-79 & $03-16-88$ \\
\hline BG 84 & 76695.9 & 67966.4 & & 274.9 & 277.3 & & 3.5" Steel & $06-01-79 \sim$ & $08-09-88$ \\
\hline BG 85 & 76719.0 & 57928.9 & & 274.8 & 276.8 & & 3.5" Steel & $06-01-79 \sim$ & $08-09-88$ \\
\hline BG 86 & 76721.4 & 67979.4 & & 275.7 & 277.7 & & 3.5" Stoel & 06-01-79 & $03-10-88$ \\
\hline BG 87 & 76748.9 & 67951.9 & & 275.4 & 277.4 & & 3.5" Stoel & 06-01-79 & $03-11-88$ \\
\hline BG 88 & 76947.7 & 58169.0 & & 281.3 & 283.3 & & 4" Btoel & 06-01-79 & $12-07-87$ \\
\hline BG 89 & 76987.9 & 68198.1 & & 281.6 & 289.8 & & $4^{\prime \prime}$ Steel & $08-01-79$ - & $12-01-87$ \\
\hline BG 90 & 76996.6 & 68162.6 & & 281.2 & 283.1 & & $4^{n}$ Steel & $05-01-79$ - & $12-08-87$ \\
\hline BG 91 & 78031.3 & 56649.4 & $236.4-200.4$ & 270.9 & 273.4 & 68.0 & PVC & $10-06-81$ & \\
\hline BG 92 & 79019.6 & 56828.0 & 227.2-197.2 & 252.7 & 255.2 & 58.0 & PVC & $10-08-81$ & \\
\hline BG 93 & 79990.8 & $57: 80.8$ & $210.6-180.6$ & 256.0 & 258.6 & 78.0 & PVC & $10-12-81$ & \\
\hline BG 94 & 80867.2 & 57484.0 & $182.8-162.8$ & 248.3 & 260.8 & 98.0 & PVC & $10-14-81$ & \\
\hline BG 98 & 80059.9 & 58407.0 & 182.5-162.6 & 220.0 & 222.5 & 70.0 & PVC & $10-19-81$ & \\
\hline BG 96 & 79996.3 & 68297.8 & 207.2-177.2 & 242.7 & 245.2 & 68.0 & Stoel & $09-26-81$ & \\
\hline BG 97 & 78474.7 & 57827.6 & 219.1-199.1 & 276.7 & 277.1 & 78.0 & $4^{n} \mathrm{Al}$ & $11-01-81$ & $01-09-89$ \\
\hline BG 98 & 77697.9 & 57398.7 & 242.6-212.6 & 278.9 & 280.5 & 68.0 & $4^{\prime \prime}$ Stoel & $09-26-81$ & $04-05-88$ \\
\hline BG 99 & 76904.6 & 58404.1 & 246.9-215.9 & 284.1 & 285.9 & 70.0 & $4^{n}$ Stoel & 10.21 .81 & $03-25-88$ \\
\hline BG 100 & 77816.6 & 68899.1 & 235.3-203.3 & 271.3 & 273.3 & 70.0 & 4" Bteel & $09-22-81$ & $02-18-88$ \\
\hline BG 101 & 78740.8 & 69277.1 & $191.4-161.4$ & 228.9 & 231.4 & 70.0 & Bteol & $09-80-81$ & \\
\hline BG 108 & 77883.6 & 59752.1 & $199.6-169.6$ & 237.0 & 239.6 & 70.0 & PVC & $10-02-81$ & \\
\hline BG 104 & 77038.8 & 69888.0 & 246.8.215.8 & 283.3 & 285.8 & 70.0 & Btoel & $09-21-81$ & \\
\hline BG 105 & 76123.8 & 69472.8 & 252.9-222.9 & 289.4 & 290.9 & 68.0 & 4" Btoel & 09.18 .81 & $12-22-88$ \\
\hline BG 106 & 75187.4 & 59162.4 & 269.6-229.5 & 288.0 & 287.5 & 68.0 & 4" Btoel & $09-17-81$ & 01-05-89 \\
\hline BG 107 & 74803.6 & 60120.1 & 228.9-208.3 & 246.8 & 248.3 & 40.0 & Stoel & $03-10-81$ & \\
\hline BG 108 & 74383.0 & 69827.9 & $247.3-217.3$ & 264.8 & 267.3 & 50.0 & Btod & $09-16-81$ & \\
\hline BG 109 & 79926.2 & 59626.1 & 258.4-228.4 & 289.9 & 286.4 & 68.0 & $4^{n}$ Btoel & $09-16-81$ & \\
\hline BG 110 & 73354.7 & 69277.2 & 254.3-224.3 & 291.8 & 294.3 & 70.0 & $4^{n}$ Bteel & $10-20-81$ & \\
\hline BG 111 & 76510.0 & 58941.0 & 236.1-216.1 & 292.4 & 294.1 & 78.0 & $4^{\prime \prime}$ Btoel & & $01-04-89$ \\
\hline BG 112 & 76969.1 & 59168.8 & 238.3-218.3 & 294.7 & 296.3 & 78.0 & 4" Stoel & & $01-05-89$ \\
\hline BG 113 & 77410.2 & 69386.0 & 216.4-196.4 & 262.7 & 264.4 & 68.0 & $4^{n}$ Bteel & & $02-19-88$ \\
\hline BG 114 & 77273.3 & 58779.6 & 227.9-207.9 & 283.8 & 285.9 & 78.0 & 4" Steel & & $02-08-89$ \\
\hline BG 115 & 77207.2 & 57884.5 & 218.9-198.9 & 275.0 & 276.9 & 78.0 & 4" Bteel & & $03-28-88$ \\
\hline BG 116 & 77584.9 & 58225.9 & $237.8-217.8$ & 295.1 & 297.8 & 80.0 & $4^{n} \mathrm{Al}$ & & $01-12-89$ \\
\hline BG 117 & 77940.1 & 58595.9 & 222.5-202.5 & 278.6 & 280.5 & 78.0 & $4^{n}$ Steel & & $01-12-89$ \\
\hline BG 118 & 78043.1 & 57628.2 & $234.4-214.4$ & 290.3 & 292.4 & 78.0 & $4^{n}$ Btoel & & $01-06-89$ \\
\hline
\end{tabular}

(eories continued) 
BG WELL SERIES (cont.)

\begin{tabular}{|c|c|c|c|c|c|}
\hline \multirow[b]{2}{*}{ Well ID } & \multicolumn{2}{|c|}{ BRS Conrdinates } & \multicolumn{3}{|c|}{ Elevations ( $\mathrm{ft}$ MSL) } \\
\hline & North & East & Screen Zone & Grnd. & Cagin: \\
\hline 19 & 77743.7 & 67004.9 & 229.2-209.2 & 285.4 & 287.2 \\
\hline BG & 78319.9 & 57199.2 & 219.2-199.2 & 274.9 & 277.2 \\
\hline BG 121 & 78760.1 & 57359.2 & 239.9-219.9 & 276.1 & 277.9 \\
\hline 122 & 78681.1 & 66789.7 & 209.9-189.9 & 246.0 & 247.9 \\
\hline & 77254.0 & 57095.0 & $234.8-214.8$ & 291.2 & 292.8 \\
\hline BG 125 & 77428.7 & 66778.1 & 236.8-216.8 & 293.3 & 291.8 \\
\hline
\end{tabular}

Eff. Well

Depth (ft) Casing Installed Abandoned

$\begin{array}{ll}78.0 & 4^{\prime \prime} \text { Steel } \\ 78.0 & 4^{\prime \prime} \mathrm{Al} \\ 58.0 & \text { Steel } \\ 68.0 & \text { Steel } \\ 78.0 & 4^{\prime \prime} \text { Steel } \\ 78.0 & \text { Steel }\end{array}$

()4-05.88

01-10-89

04-06-88

BG WELL SERIES (cont.)

Description: Burial Grounds Grid Wells

Bldg. Number: 643-7E, -28E

Map: Not applicable

\begin{tabular}{|c|c|c|c|c|c|c|c|c|}
\hline \multirow[b]{2}{*}{ Well ID } & SRS Coordinates & \multicolumn{3}{|c|}{ Elevations (ft MSL) } & \multirow{2}{*}{$\begin{array}{l}\text { Dff. Well } \\
\text { Depth (f) }\end{array}$} & \multirow[b]{2}{*}{ Casing } & \multirow[b]{2}{*}{ Inotalled } & \multirow[b]{2}{*}{ Abandonod } \\
\hline & North East & Screen Zone & Grnd. & $\overline{\text { Casing }}$ & & & & \\
\hline BG 204GR & & & 303.4 & 306.9 & & 4" Stoel & $07.09-80$ & $06-17-87$ \\
\hline BG 206GR & & & 298.6 & 301.1 & & 4" Stoel & $12-22-80$ & $05-21-87$ \\
\hline BG 208GR & & & 293.9 & 296.4 & & 4" Bteel & $12-16-80$ & $06-18-87$ \\
\hline BG 210GR & & & 291.5 & 294.0 & & $4^{n}$ Bteel & $10-09-80$ & $05-12-87$ \\
\hline BG $212 G R$ & & & 285.4 & 287.9 & & $4^{\prime \prime}$ Stoel & $12-11.80$ & $0,5-06-87$ \\
\hline BG 214GR & & & 283.6 & 286.0 & & $4^{n}$ Steel & $07-11-83$ & $08-23-87$ \\
\hline BG 216GR & & & 282.7 & 285.2 & & $4^{\prime \prime}$ Bteel & $07-24-6 i$ & $06-26-87$ \\
\hline B/G 218GR & & & 282.6 & 285.1 & & 4" Bteel & $07-30.80$ & $01-13-88$ \\
\hline BG 220GR & & & 283.2 & 285.7 & & $4^{n}$ Steel & $08-04-80$ & $06-11-87$ \\
\hline BG 222GR & & & 277.9 & 280.4 & & $4^{\prime \prime}$ Btoel & $12-10-80$ & $11-18-88$ \\
\hline BG 224GR & & & 276.6 & 279.1 & & Stoel & $08-19-80$ & \\
\hline BG 402GR & & & 301.6 & 304.1 & & $4^{\prime \prime}$ Bteel & $09-08-80$ & $04-14-87$ \\
\hline BG 404GR & & & 302.6 & 305.1 & & $4^{n}$ Btes! & $09-12-80$ & $04-20-87$ \\
\hline BG 406GR & & & 297.8 & 800.3 & & $4^{n}$ Bteel & $09-16-80$ & $04-24-87$ \\
\hline BG 408GR & & & 290.8 & 293.9 & & 4" Stoel & $09-24-80$ & $05-01-87$ \\
\hline BG 410GR & & & 287.0 & 289.6 & & 4" Bteol & $12-04-80$ & $06-27-87$ \\
\hline BG 420GR & & & 287.4 & 289.9 & & $4^{n}$ Bteel & 11.19 .80 & $11-29-88$ \\
\hline BG 422GR & & & 279.5 & 282.0 & & $4^{n}$ Btoel & $11-25-80$ & $11-21-88$ \\
\hline BG 424GR & & & 275.4 & 277.9 & & Steel & $08-18-80$ & \\
\hline BG 620GR & & & 289.0 & 291.6 & & $4^{\prime \prime}$ Bteel & $10-27-80$ & $08-01-87$ \\
\hline BG 622GR & & & 282.7 & 285.2 & & $4^{\infty}$ Steel & $11-21.80$ & $11-16-88$ \\
\hline BG 624GR & & & 277.2 & 279.7 & & Stoel & $08-20-80$ & \\
\hline BG 818GR & & & 284.8 & 287.3 & & $4^{n}$ Btoel & $11-12-80$ & $04-02-87$ \\
\hline BG 820GR & & & 286.4 & 288.9 & & $4^{n}$ Stoel & $11-05-80$ & $04-09-87$ \\
\hline BG 822GR & & & 283.1 & 285.6 & & $4^{n}$ Btoel & 11.07 .80 & $01-13-88$ \\
\hline BG 824GR & & & 278.6 & 281.1 & & Bteel & $08-28-80$ & \\
\hline
\end{tabular}

Notes: These wells have been reported in some SRS reports prior to 1988 in abbreviated form (i.e., BG 204GR $=22.04$, BG Q2AGR = 28.24). 


\section{BG-I Well}

Description: Unofficial well in F Area

Bldg. Number: Not applicable

Map: Figure A-11

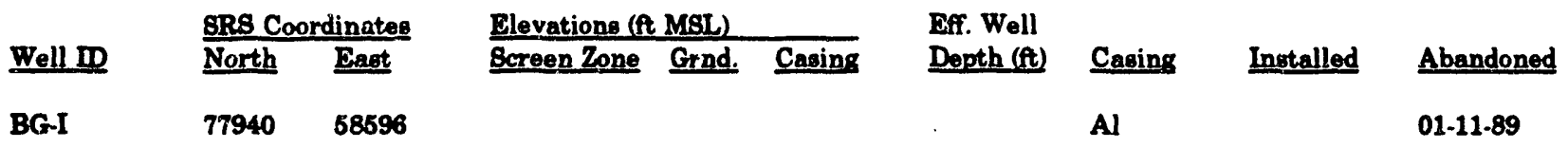

Notes: The iCentification of this well comes from the abandonment sheets. The BG-I designation is unoficial. According to the Well Abandonment Report, this well did not have a screen.

\section{BGO WELL SERIES}

Description: Burial Grounds Perimeter Wells

Bldg. Number: 643-7E, -28E

Map: Figures A-8, A-10, A-12, and A-13

\begin{tabular}{|c|c|c|c|c|c|c|c|c|c|}
\hline \multirow[b]{2}{*}{ Well $\mathbf{m}$} & \multicolumn{2}{|c|}{ SRS Coordinatos } & \multicolumn{3}{|c|}{ Hlovations (A MBL) } & \multirow{2}{*}{$\begin{array}{l}\text { Def. Well } \\
\text { Depth (t) }\end{array}$} & \multirow[b]{2}{*}{ Caring } & \multirow[b]{2}{*}{ Installed } & \multirow[b]{2}{*}{ Abandoned } \\
\hline & North & Eect & Ecreen Zone & Gred. & Cacing & & & & \\
\hline BGO ID & 79737.9 & 68779.3 & 246.0 .225 .0 & 298.0 & 295.1 & 70.1 & 4" PVC & $02-10-87$ & \\
\hline BCO 2D & 74652.9 & 58809.7 & 238.9-218.9 & 294.9 & 298.9 & 78.0 & 4" PVC & $0812-87$ & \\
\hline BGO 3D & 76851.3 & 68809.2 & $247.6-227.6$ & 290.8 & 292.7 & 66.1 & $4^{*}$ PVC & $0210-87$ & \\
\hline BCO $4 D$ & 78160.1 & 68808.7 & $240.6-220.6$ & 296.6 & 297.6 & 76.9 & 4" PVC & $08-13-87$ & \\
\hline BGO $5 \mathrm{C}$ & 76476.9 & 58794.6 & 193.2-183.2 & 294.2 & 296.1 & 112.9 & 4" PVC & $09-04-87$ & \\
\hline BGO $5 D$ & 76477.5 & 58784.8 & 239.3-219.3 & 294.2 & 296.3 & 77.0 & 4" PVC & $0904-87$ & \\
\hline BGO 6A & 76487.2 & 58316.8 & 117.8-107.5 & 283.8 & 286.6 & 178.1 & ** & $10-08-87$ & \\
\hline BGO $6 \mathrm{C}$ & 76487.1 & 58307.0 & $168.0-158.0$ & 283.5 & 285.6 & 127.6 & 4" PVC & $09-02-87$ & \\
\hline BGO 6D & 76487.3 & 68297.1 & $237.2-217.2$ & 289.2 & 285.6 & 68.3 & 4" PVC & 08-31-87 & \\
\hline BGO TD & 78494.5 & 679172 & $285.5-216.5$ & 280.5 & 282.4 & 68.9 & 4" PVC & $08-16-87$ & \\
\hline BGO $8 A$ & 76669.0 & $\mathbf{5 7 6 1 8 . 3}$ & $116.3-105.3$ & 281.3 & 283.2 & 177.9 & $*$ & 10.07 .87 & \\
\hline BGO $8 A R$ & 76598.8 & 57617.5 & 104.6. 94.6 & 284.6 & 288.6 & 192.0 & $4^{\circ}$ PVC & $02-26-91$ & \\
\hline BGO $8 C$ & 76679.2 & 57618.7 & $179.8-169.8$ & 281.3 & 288.3 & 113.6 & 4" PVC & $08-28-61$ & \\
\hline BGO 80 & 76588.8 & 57617.8 & 256.1-216.1 & 281.1 & 283.2 & 67.1 & 4 PVC & $08-28-87$ & \\
\hline BGO 9D & 76811.6 & 67478.9 & $229.2-209.2$ & 289.2 & 285.1 & 78.9 & 4" PVC & $08-20-87$ & \\
\hline BGO 10A & 76806.2 & 57000.9 & 121.1-111.1 & 299.1 & 500.9 & 189.8 & ** & $10-06-87$ & \\
\hline BGO 10C & 76806.2 & 57041.1 & $167.3-167.3$ & 299.3 & 301.3 & 144.0 & 4" PVC & $09-16-87$ & \\
\hline BGO 10D & 76806.1 & 57030.6 & $260.6-250.6$ & 299.5 & 301.6 & 71.0 & 4" PVC & $09-16-87$ & \\
\hline BGO 10DR & 76804.8 & 57073.7 & $238.3-218.3$ & 298.3 & 300.4 & 82.1 & 4" PVC & $02-08-91$ & \\
\hline BGO 11D & 76806.1 & 56651.3 & 236.3-216.3 & 303.3 & 306.3 & 89.0 & 4" PVC & $08-24-87$ & \\
\hline BGO $12 A$ & 76804.6 & 66250.7 & $116.4-108.4$ & 311.4 & 313.4 & 207.0 & 4" PVC & $10-02-87$ & \\
\hline BGO 12C & 76806.2 & 66241.1 & $163.6-163.6$ & 311.6 & 313.6 & 160.0 & 4" PVC & $10.01-87$ & \\
\hline BGO 12D & 76805.2 & 56231.1 & $237.8-217.8$ & 311.8 & 313.7 & 95.9 & 4" PVC & $09-29-87$ & \\
\hline BGO 13D & 76806.3 & 65840.0 & 248.6-228.6 & 316.6 & 318.6 & $\mathbf{9 0 . 0}$ & 4" PVC & $10-12-87$ & \\
\hline BGO 14A & 76377.5 & 56838.3 & $119.6-109.6$ & 300.2 & 301.9 & 192.3 & $* *$ & $10.19-87$ & \\
\hline BGO 14C & 76367.7 & 55839.0 & 202.1-192.1 & 300.1 & 302.0 & 109.9 & $4^{n}$ PVC & $10-16-87$ & \\
\hline BGO 14D & 76357.6 & 55839.6 & $249.6-229.6$ & 300.0 & 302.0 & 72.4 & 4" PVC & $10.16-87$ & \\
\hline BGO 16D & 75973.6 & 55869.1 & $258.7-218.7$ & 296.7 & 298.7 & 80.0 & 4" PVC & $08-25-87$ & \\
\hline BGO 18A & 75757.0 & 66194.2 & 112.5-102.6 & 302.8 & 305.0 & 202.5 & ** & $12-08-87$ & \\
\hline BGO 16D & 75751.4 & 56202.1 & $237.3-217.3$ & 302.3 & 304.6 & 87.3 & 4" PVC & $08-24-87$ & \\
\hline BGO $17 \mathrm{D}$ & 76599.6 & 66399.4 & $224.0-204.0$ & 296.0 & 298.3 & 94.3 & 4" PVC & $07-08-87$ & \\
\hline BGO 18A & 75599.9 & 66699.7 & 109.5-99.5 & 292.9 & 295.2 & 195.7 & $*$ & $10-30-87$ & \\
\hline
\end{tabular}

(eriee continued) 


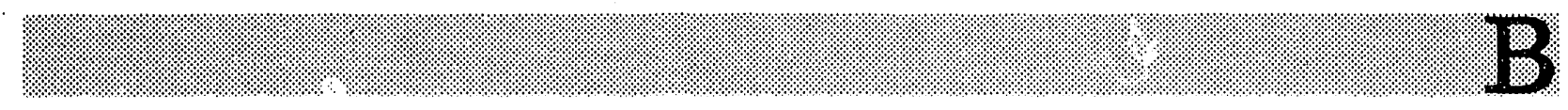

BGO WELL SERIES (cont.)

\begin{tabular}{|c|c|c|c|c|c|c|c|c|c|}
\hline & SRS Coo & linates & Elevationg (f & MSL) & & Eff. Well & & & \\
\hline Well ID & North & East & Bcreen Zone & Grnd. & Casing & Depth (t) & Casing & Installed & Abandoned \\
\hline BGO 18D & 75600.0 & 56711.2 & 239.6-219.6 & 292.6 & 294.9 & 75.3 & 4" PVC & $11-04-87$ & \\
\hline BGO 19D & 75350.0 & 66997.3 & 213.9-193.9 & 284.9 & 287.2 & 93.3 & 4" PVC & $07-15-87$ & \\
\hline BGO 20D & 74962.2 & 57113.8 & 236.3-216.3 & 281.3 & 283.7 & 67.4 & 4" PVC & $03-02-88$ & \\
\hline BGO $21 D$ & 74688.6 & 57470.7 & $237.7-217.7$ & 283.0 & 285.4 & 67.7 & $4^{n}$ PVC & $11-11-87$ & \\
\hline BGO 22D & 74482.2 & 57817.3 & 214.2-194.2 & 284.2 & 286.6 & 92.3 & $4^{n}$ PVC & $07.23-87$ & \\
\hline BGO 23D & 74238.1 & 68133.0 & $242.0-222.0$ & 287.0 & 289.2 & 67.2 & 4" PVC & $08-06-87$ & \\
\hline BGO 24D & 74012.4 & 58438.8 & $241.0-221.0$ & 291.0 & 293.2 & 72.2 & 4" PVC & $07-29-87$ & \\
\hline BGO 25A & 76158.5 & 55668.1 & 114.1-104.1 & 294.7 & 296.5 & 192.4 & $*$ & $10-19-87$ & \\
\hline BGO 26A & 76144.6 & 55014.2 & 91.0 .81 .0 & 285.1 & 287.2 & 206.2 & $4^{n}$ PVC & $03-16-89$ & \\
\hline$B G C$ 26D & 76128.0 & 55015.2 & 233.6-213.4 & 283.5 & 285.5 & 72.1 & $4^{\prime \prime}$ PVC & $12.02-88$ & \\
\hline $\mathrm{BGO} 27 \mathrm{C}$ & 75666.3 & 54671.4 & 163.9-164.9 & 273.9 & 276.0 & 121.1 & 4" PVC & $03-17-89$ & \\
\hline BCO 27D & 75677.3 & 54680.2 & $229.3-209.3$ & 974.3 & 276.3 & 67.0 & $4^{n}$ PVC & $12-05-88$ & \\
\hline BGO 28D & 7534.8 .3 & 54467.9 & 230.1-210.1 & 275.1 & 277.4 & 67.3 & $4^{n}$ PVC & $12-16-88$ & \\
\hline BGO 29A & 75660.0 & 54103.5 & 112.6-102.5 & 262.1 & 264.2 & 161.7 & $4^{\prime \prime}$ PYC & $02-17-89$ & \\
\hline BGO 30C & 75181.0 & 54512.3 & $188.4-178.4$ & 272.6 & 274.5 & 96.1 & $4^{\prime \prime}$ PVC & $04-05.89$ & \\
\hline BGO 30D & 76187.7 & 54499.2 & $227.8-207.8$ & 272.8 & 274.8 & 67.0 & $4^{\prime \prime}$ PVC & $02-06-89$ & \\
\hline BGO 31C & 74978.0 & 54816.2 & $186.4-176.4$ & 271.1 & 273.1 & $\mathbf{9 6 . 7}$ & $4^{*}$ PVC & 04-05-89 & \\
\hline BGO 31D & 74986.3 & 54841.7 & 231.1-211.1 & 271.6 & 273.7 & 62.6 & 4" PVC & 02-11-89 & \\
\hline BGO 32D & 74727.0 & 55250.2 & 234.6-214.5 & 279.5 & 281.7 & 67.2 & $4^{n}$ PVC & $12-16-88$ & \\
\hline RGO 3SC & 74479.7 & 65681.4 & $187.8-177.8$ & 277.4 & 279.4 & 101.6 & $4^{n}$ PVC & $04-04-89$ & \\
\hline BGO 33D & 74468.7 & 55696.4 & 233.1-213.1 & 278.1 & 280.3 & 67.2 & $4^{*}$ PVC & $12-06-88$ & \\
\hline BGO 34D & 74228.8 & 56082.6 & 232.7-212.7 & 272.7 & 274.9 & 62.2 & $4^{n}$ PVC & $12-07-88$ & \\
\hline BGO 36C & 73353.9 & 56546.7 & 171.9-161.9 & 271.4 & 273.4 & 111.6 & $4^{\prime \prime}$ PVC & $04-03.89$ & \\
\hline BGO 35D & 73946.0 & 66556.5 & 239.4-219.4 & 271.4 & 279.5 & 54.1 & $4^{n}$ PVC & $12-08-89$ & \\
\hline BGO S6D & 73749.8 & 56888.1 & 243.3-223.3 & 273.3 & 275.4 & 52.1 & $4^{\prime \prime}$ PVC & $12-08-88$ & \\
\hline BGO 37C & 73498.2 & 67279.2 & $178.8-168.8$ & 284.3 & 286.3 & 117.6 & $4^{n}$ PVC & $03-31-89$ & \\
\hline BGO S7D & 73490.8 & 67292.9 & 246.1-226.1 & 286.1 & 287.3 & 61.2 & $4^{*}$ PVC & $12-09-88$ & \\
\hline BGO SBD & 73399.3 & 67557.6 & 242.3-222.3 & 289.3 & 291.6 & 69.3 & $4^{n}$ PVC & $12-16-88$ & \\
\hline BGO S9D & 73683.6 & 67891.0 & 244.7-224.7 & 293.7 & 295.7 & 71.0 & $4^{\prime \prime}$ PVC & $12-14-88$ & \\
\hline BGO $40 D$ & 76125.8 & 84638.6 & 226.6-216.6 & 286.4 & 288.4 & 71.8 & $4^{n}$ PVC & $03-14-91$ & \\
\hline BGO 45A & 75830.0 & 64560.1 & $126.9-116.9$ & 276.9 & 278.9 & 162.0 & $4^{\prime \prime}$ PVC & $03-08-91$ & \\
\hline BGO $45 B$ & 75840.3 & 54569.6 & $147.0-197.0$ & 276.6 & 278.6 & 141.6 & $4^{n}$ PVC & $03-08-91$ & \\
\hline BGO $45 C$ & 75835.0 & 54577.4 & $200.6-190.6$ & 276.5 & 278.6 & 88.1 & $4^{n}$ PVC & $03-11-91$ & \\
\hline BGO ASD & 75854.3 & 54585.6 & 229.6-209.6 & 276.6 & 278.6 & 69.0 & 4" PVC & $03-14-91$ & \\
\hline
\end{tabular}

Notes: BCO 18A was originally installed 9-11-87. This well was superseded by another well with the same name, inotalled 12-08.87.

* BGO 6A, 8A, 10A, 14A, 16A, 18A, and 25A have 4-in. PVC casing inside 12-in. otoel casing. All screens are PVC.

\section{BGX WELL SERIES}

Description: E-Area Vaults near the Burial Grounds

Bldg. Number:

Map: Figures A-8 and A-10

\begin{tabular}{|c|c|c|c|c|c|c|c|c|c|}
\hline \multirow[b]{2}{*}{ Well ID } & \multicolumn{2}{|c|}{ SR8 Coordinates } & \multicolumn{3}{|c|}{ Elevations (ft MSL) } & \multirow{2}{*}{$\begin{array}{l}\text { Eff. Well } \\
\text { Depth (f) }\end{array}$} & \multirow[b]{2}{*}{ Casing } & \multirow[b]{2}{*}{ Installed } & \multirow[b]{2}{*}{ Abandoned } \\
\hline & North & East & Screen Zone & Grnd. & Casing & & & & \\
\hline $\begin{array}{ll}\text { BGX } & 2 B \\
B G X & 2 D\end{array}$ & $\begin{array}{l}77203.4 \\
77192.4\end{array}$ & $\begin{array}{l}58256.5 \\
58265.6\end{array}$ & $\begin{array}{l}147.2-137.2 \\
191.1-181.1\end{array}$ & $\begin{array}{l}289.2 \\
289.1\end{array}$ & $\begin{array}{l}291.3 \\
291.1\end{array}$ & $\begin{array}{l}154.1 \\
110.0\end{array}$ & $\begin{array}{l}4^{n} \text { PVC } \\
4^{n} \text { PVC }\end{array}$ & $\begin{array}{l}02-15-91 \\
03-01.91\end{array}$ & \\
\hline
\end{tabular}

(series continued) 
BGX WELL SERIES (cont.)

\begin{tabular}{|c|c|c|c|c|c|c|c|c|c|}
\hline \multirow{2}{*}{ Well ID } & \multicolumn{2}{|c|}{ BRB Coordinates } & \multicolumn{3}{|c|}{ Flevations (At MSL) } & \multirow{2}{*}{$\begin{array}{l}\text { Eff. Well } \\
\text { Depth (A) }\end{array}$} & \multirow[b]{2}{*}{ Caging } & \multirow[b]{2}{*}{ Inetalled } & \multirow[b]{2}{*}{ Abandoner } \\
\hline & North & Fast & Bcreen Zone & Grnd. & Caning & & & & \\
\hline BGX $3 D$ & 77677.0 & 57780.1 & 221.6-201.6 & 289.1 & 291.2 & 89.6 & 4" PVC & $02-07-91$ & \\
\hline BGX 4A & 77879.2 & 57216.6 & $116.8-106.8$ & 288.8 & 290.9 & 184.1 & $4^{n}$ PVC & $01-22-91$ & \\
\hline$B G X$ 4C & 77886.2 & 67202.2 & 180.7-170.7 & 288.7 & 890.8 & 120.1 & 4" PVC & $01-29.91$ & \\
\hline BGX 4D & 77898.9 & 67186.2 & 223.8-203.8 & 288.8 & 290.9 & 87.1 & $4^{n} \mathrm{PVC}$ & $01-29-91$ & \\
\hline BGX ED & 78402.0 & 67308.6 & 216.0.195.0 & 283.0 & 285.0 & 90.0 & $4^{n} \mathrm{FVC}$ & $02-06-91$ & \\
\hline BGX 9D & 76986.0 & 69592.1 & 292.4-212.4 & 277.4 & 279.4 & 67.0 & $4^{*}$ PVC & $03-18-91$ & \\
\hline BGX 10D & 76183.3 & 69786.6 & 236.2-216.2 & 274.8 & 276.9 & 60.7 & 4" PVC & $02-06-91$ & \\
\hline BGX $12 C$ & 74427.9 & 69676.8 & $184.1-174.1$ & 273.1 & 276.1 & 101.0 & $4^{n}$ PVC & 01.21 .91 & \\
\hline BGX 12D & 74410.9 & 69674.3 & 243.7-223.7 & 273.2 & 275.2 & 51.6 & 4" PVC & $01-21-91$ & \\
\hline
\end{tabular}

Notis: These wells were initially named the Burial Grounds Expansion wells.

RGX 2D, 1D, and $\mathbf{6 D}$ are water table wells screened within Aquifer Unit $\mathrm{IB}_{1}$ (McBean formation).

\section{BH WELL SERIES}

Description: S-Area Defense Waste Processing Facility (DWPF) Boreholes and Piezometers Bldg. Number: Not applicable Map: Figures A-15 and A-16

\begin{tabular}{|c|c|c|c|c|c|c|c|c|c|}
\hline \multirow[b]{2}{*}{ Well ID } & \multicolumn{2}{|c|}{ BRA Coondinates } & \multicolumn{3}{|c|}{ Mlevations (At MSI) } & Exf. Woll & \multirow[b]{2}{*}{ Coning } & \multirow[b]{2}{*}{ Inatalled } & \multirow[b]{2}{*}{ Abandone } \\
\hline & North & Eant & Erreen 7one & Grnd & Caring & Deph (t) & & & \\
\hline 2 & 74900 & 64000 & (46.6- 49.1) & 280.9 & 260.9 & $\$ 10.0$ & $\omega$ & $02-07-78$ & $01-19-87$ \\
\hline $\mathbf{s}$ & 76008 & 64601 & 168.9-161.4 & 276.4 & 276.4 & 125.0 & $1.25^{\circ}$ PVC & $02-18-78$ & $10-16-87$ \\
\hline 4 & 76108 & 65306 & 97.5. 96.0 & 289.9 & 286.0 & 190.0 & $1.25^{\circ} \mathrm{PVC}$ & $02-10.78$ & $10-13-87$ \\
\hline 13 & 72999 & 64002 & (1.0. 3.6) & 300.2 & 306.6 & $\mathbf{3 1 0 . 0}$ & $+\infty$ & $02-08-78$ & $02-06-88$ \\
\hline 18 & 72992 & 66008 & $103.7-101.2$ & 268.1 & 271.2 & 170.0 & $1.25^{\mathrm{n}}$ PVC & $02-22-78$ & $01-27-88$ \\
\hline EOA & 74690 & 66790 & (28.6-28.1) & 282.9 & 286.9 & 312.0 & & & $10.28-87$ \\
\hline I $64 A$ & 74406 & 65790 & & & & & & & $10.23-8 T$ \\
\hline & 74231 & 66787 & 225.6-223.0 & 274.0 & 276.4 & 69.4 & $1.26^{\circ} \mathrm{PVC}$ & & $10-19-87$ \\
\hline
\end{tabular}

Notes: The BR Beries has aleo been known as the DWP Beries.

The dates in the Installed column for $\mathrm{BA}$ 2, 3, 4, 18, and 16 are the lact dates that cores wore logged.

EAT 2, 13, c0A, and e4A have 1.25-in. PVC casing inside 4-in. PVC casing.

\section{BRD WELL SERIES}

Description: Road A Chemical Basin (Baxley Road)

Bldg. Number: 904-111G

Map: Figure A-26

\begin{tabular}{|c|c|c|c|c|c|c|c|c|c|}
\hline & SRs Co & dinates & Elovations & M(s) & & Eff. Well & & & \\
\hline Well ID & North & Fast & Bereen Zone & Grnd. & Casing & Depth (tt) & Caning & Installed & Abandoned \\
\hline BRD 1 & 55860.0 & 29277.7 & $178.9-148.9$ & 203.9 & 206.8 & 66.9 & $4^{\prime \prime}$ PVC & $05-10-83$ & \\
\hline
\end{tabular}

(cories continued) 
BRD WELL SERIES (cont.)

\begin{tabular}{|c|c|c|c|c|c|c|c|c|c|}
\hline \multirow[b]{2}{*}{ Well ID } & \multicolumn{2}{|c|}{ BRS Coordinaten } & \multicolumn{3}{|c|}{ Elevations (ft MSL) } & \multirow{2}{*}{$\begin{array}{l}\text { Eff. Well } \\
\text { Depth (ft) }\end{array}$} & \multirow[b]{2}{*}{ Casing } & \multirow[b]{2}{*}{ Installed } & \multirow[b]{2}{*}{ Abandoned } \\
\hline & North & East & Screen Zone & Grnd. & Casing & & & & \\
\hline BRD 2 & 56093.3 & 29357.1 & $178.5-148.5$ & 205.5 & 207.3 & 58.8 & 4" FVC & $05-13-83$ & \\
\hline BRD 3 & 65918.7 & 29538.9 & 188.5-158.5 & 218.5 & 220.4 & 61.9 & 4" PVC & $05-16-83$ & \\
\hline BRD 4 & 56060.4 & 29219.2 & 159.1-129.1 & 196.1 & 197.9 & 68.8 & 4" PVC & $07-16-84$ & \\
\hline BRD ED & 55955.7 & 29252.6 & $168.4-148.4$ & 202.7 & 205.0 & 56.6 & $4^{n}$ PVC & $07-11-89$ & \\
\hline
\end{tabular}

\section{BRR WELL SERIES}

Description: Burma Road Rubble Pit

Bldg. Number: 231-4F

Map: Figures A-12 and A-13

\begin{tabular}{|c|c|c|c|c|c|c|c|c|c|}
\hline \multirow[b]{2}{*}{ Well ID } & \multicolumn{2}{|c|}{ SRS Coordinates } & \multicolumn{3}{|c|}{ Elevations (ft MSL) } & \multirow{2}{*}{$\begin{array}{l}\text { Bff. Well } \\
\text { Depth (ft) }\end{array}$} & \multirow[b]{2}{*}{ Casing } & \multirow[b]{2}{*}{ Installed } & \multirow[b]{2}{*}{ Abandoned } \\
\hline & North & East & Screen Zone & Grnd. & Casing & & & & \\
\hline BRR 1D & 77365.2 & 50588.2 & 220.4-200.4 & 293.8 & 295.9 & 96.5 & 4" PVC & 02-09-90 & \\
\hline BRR 2D & 77431.4 & 60306.J & 216.1-196.1 & 289.6 & 291.9 & 95.8 & 4" PVC & 02-09-90 & \\
\hline BRR 3D & 77398.3 & 50203.6 & 217.1-197.1 & 289.6 & 291.7 & 94.6 & 4" PVC & $02-12-90$ & \\
\hline BRR 4D & 77960.5 & 50104.5 & 218.7-198.7 & 290.0 & 292.2 & 93.6 & 4" PVC & $02-02-90$ & \\
\hline ERR 6D & 77266.7 & 50009.0 & 222.1-202.1 & 292.6 & 294.6 & 92.6 & 4" PVC & $01-30-90$ & \\
\hline
\end{tabular}

\section{BTH WELL SERIES}

Description: Bentonite Test Pit Wells, across from the entrance to the Burial Grounds on Road E

Bldg. Number: 724-7G

Map: Figure A.9

\begin{tabular}{|c|c|c|c|c|c|c|c|c|}
\hline \multirow[b]{2}{*}{ Well ID } & \multicolumn{2}{|c|}{ BRs Coordinates } & \multicolumn{2}{|l|}{ Elevations (ft MSL) } & \multirow{2}{*}{$\begin{array}{l}\text { Eff. Well } \\
\text { Depth (A) }\end{array}$} & \multirow[b]{2}{*}{ Casing } & \multirow[b]{2}{*}{ Installed } & \multirow[b]{2}{*}{ Abandoned } \\
\hline & North & East & Scresn Zone Grnd. & Casing & & & & \\
\hline BTH 1 & 73847.1 & 54697.3 & & & & $3^{n} \mathrm{Al}$ & 1965 & $04-27-88$ \\
\hline BTH 2 & 73829.1 & 54715.6 & & & & $3.5^{\mathrm{n}} \mathrm{Al}$ & 1965 & $04-27-88$ \\
\hline BTH 3 & 73908.8 & 54733.8 & & & & 3.5" Al & 1965 & $04-28-88$ \\
\hline BIIH 4 & 73792.1 & 54753.1 & & & & $3^{n} \mathrm{Al}$ & 1965 & $04-27.88$ \\
\hline BMH 6 & 73773.1 & 54772.6 & & & & $3.5^{\circ} \mathrm{Al}$ & 1986 & $04-26-88$ \\
\hline BIM 6 & 73755.6 & 54789.2 & & & & $3.5^{\prime \prime} \mathrm{Al}$ & 1965 & $04-26-88$ \\
\hline BTH 7 & 73718.3 & 64783.2 & & & & 3.5" Al & 1965 & $04-26-88$ \\
\hline BIYH 8 & 73758.0 & 54744.0 & & & & $3.5^{n} \mathrm{Al}$ & 1965 & $04-27-88$ \\
\hline BIH 9 & 73775.7 & 54748.9 & & & & $3^{n} \mathrm{Al}$ & 1965 & $04-27-88$ \\
\hline BIH 10 & 73800.2 & $54708.1 i$ & & & & $1.5^{n} \mathrm{Al}$ & 1965 & $04-28-88$ \\
\hline BTH 11 & 73802.0 & 54686.6 & & & & $3.6^{\mathrm{n}} \mathrm{Al}$ & 1965 & $04-26-88$ \\
\hline BIH 12 & & & & & & Al & 1965 & $05-02-88$ \\
\hline BTH 13 & 73541.2 & 54670.5 & & & & Al & 1965 & $04-28-88$ \\
\hline BTH 14 & 73573.7 & 54648.8 & & & & $3.5^{n} \mathrm{Al}$ & 1965 & $04-28-88$ \\
\hline BTH 15 & 73593.9 & 54649.7 & & & & $3.5^{n} \mathrm{Al}$ & 1965 & $05-02-88$ \\
\hline BTH 16 & 73604.0 & 54649 & & & & Al & 1965 & $05-02-88$ \\
\hline BIH 17 & 73623.6 & 54649.5 & & & & $3.5^{\prime \prime} \mathrm{Al}$ & 1965 & $04-29-88$ \\
\hline BTH 18 & 73681.9 & 54741.4 & & & & $3.5^{n} \mathrm{Al}$ & 1965 & $04-28-88$ \\
\hline
\end{tabular}

(series continued) 
BTH WELL SERIES (cont.)

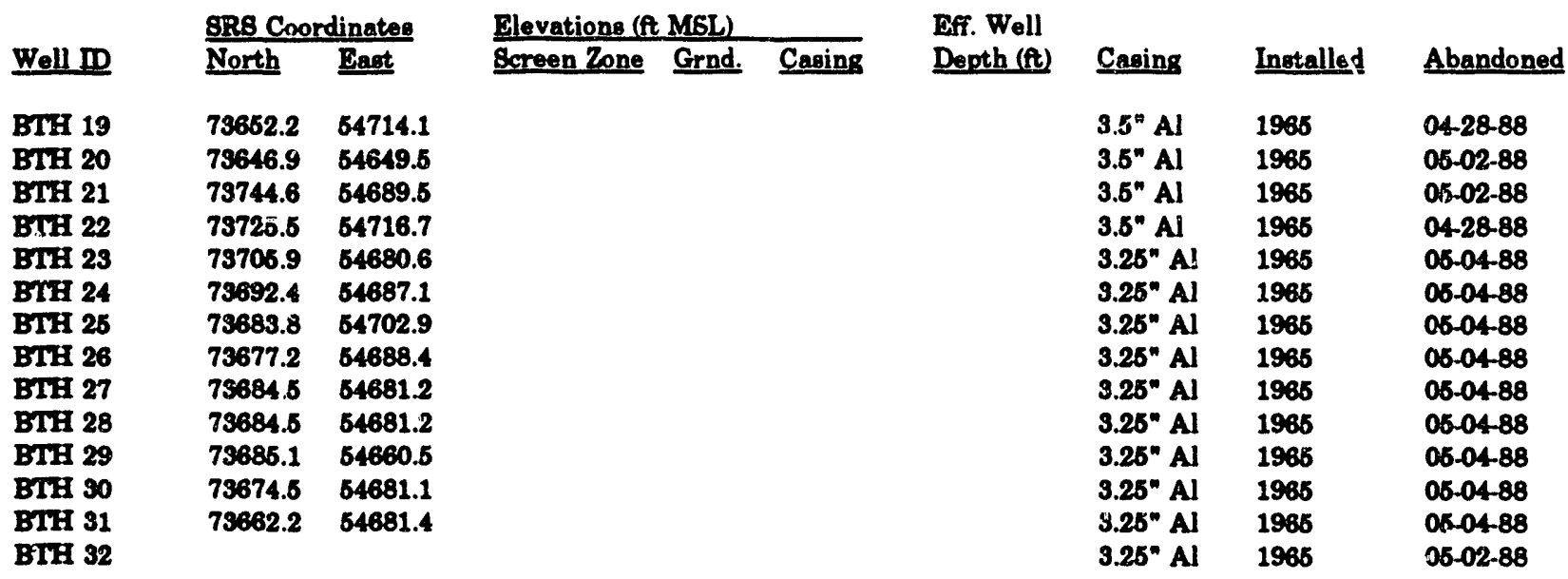

Notes: According to Hawkins and Horton (1967) the BTP welle were inotalled in the mid.1960s when "BRL pereonnel conducted a resc arch project at this site to determine if bentonite could be used as a protective cover for buried radioactive want s."

The BIII Beries was known as the BIP Beries until 1991 when the name was changed to avoid confusion with the characterization piezometers for the propoeed Banitary Landfill, Bite B, off E? Road. Bee the BIP Beries.

\section{BTP WELL SERIES}

Description: Chararterization piezometers for the proposed Sanitary Landfill, Site B, off E2 Road

Bldg. Number:

Map: Figure A-23

\begin{tabular}{|c|c|c|c|c|c|c|c|c|c|}
\hline \multirow{2}{*}{\multicolumn{2}{|c|}{ Well ID }} & \multicolumn{2}{|c|}{ ERs Coordinates } & \multicolumn{3}{|c|}{ Mlevations (ft MSI) } & \multirow{2}{*}{$\begin{array}{l}\text { Bf. Woll } \\
\text { Depth (ft) }\end{array}$} & \multirow[b]{2}{*}{ Coang } & \multirow[b]{2}{*}{ Intalled } \\
\hline & & North & Bant & Ecreen Zone & Grnd. & Canins & & & \\
\hline ETP & 1D & 74689.6 & 76186.7 & 236.1-225.1 & 293.6 & 296.9 & 71.8 & $2^{*}$ PVC & $10-09-90$ \\
\hline BTP & 1DD & 74683.6 & 75186.7 & $263.1-243.1$ & 293.6 & 298.9 & 68.8 & 1. PVC & $1009-90$ \\
\hline BTP & $2 D$ & 73421.7 & 74770.7 & $241.2-281.2$ & \$16.7 & 319.7 & 88.6 & 2" PVC & $10.08-80$ \\
\hline BIP & 2DD & 79421.7 & 74770.7 & $269.2-249.0$ & 316.7 & 319.7 & 70.7 & 1" PVC & $10-08-90$ \\
\hline BIP & 3D & 73399.7 & 76613.7 & $235.4-225.4$ & 297.9 & 301.9 & 76.5 & $2^{\infty}$ PVC & $10-08.90$ \\
\hline BIP & 3DD & 73399.7 & 75613.7 & $263.4-243.4$ & 297.9 & 301.9 & 58.6 & 1" PVC & $10-08-80$ \\
\hline BTP & $4 D$ & 72201.2 & 76004.0 & $227.9-217.9$ & 299.4 & 302.5 & 84.6 & 2* PVC & $10-08-90$ \\
\hline BTP & 4DD & 72201.2 & 76004.0 & 245.9-235.9 & 299.4 & 302.6 & 66.6 & 1" PVC & $10-08-90$ \\
\hline BTP & 5C & 77091.7 & 74098.8 & & & & & $2^{\infty}$ PVC & $11-28-90$ \\
\hline BTP & $6 \mathrm{C}$ & 76698.9 & 74402.4 & 121.7-111.7 & 30e? & 308.9 & 197.2 & 2" PVC & $09-25-90$ \\
\hline BTP & 6D & 78610.6 & 74409.3 & $230.9-220.9$ & 306.9 & 303.4 & 88.6 & $2^{n}$ PVC & $09-26-90$ \\
\hline BTP & 6DD & 76810.6 & 74409.3 & $251.9-241.9$ & 308.9 & 309.4 & 67.5 & 1" PVC & $09-25-90$ \\
\hline BIP & $7 \mathrm{C}$ & 76595.1 & 75714.4 & 101.5. 91.6 & 313.5 & 316.4 & 224.9 & $2^{\circ}$ PVC & $11-26-90$ \\
\hline BTP & $\mathbf{T D}$ & 76580.5 & 76712.8 & 228.6-218.6 & 313.6 & 317.2 & 98.6 & $2^{n}$ PVC & $1204-90$ \\
\hline BTP & TDD & 76580.5 & 76712.8 & 246.6-236.6 & 313.6 & 316.7 & 80.1 & $1^{\circ} \mathrm{PVC}$ & $12-04-9$ \\
\hline BTP & $8 \mathrm{C}$ & 75796.6 & 76202.2 & $116.9-106.8$ & 321.8 & 323.3 & 216.5 & 2" PVC & $08-29-90$ \\
\hline STP & $8 D$ & 76813.7 & 75226.6 & 232.7-222.7 & 322.7 & 324.9 & 102.2 & $2^{n}$ PVC & $08-29.9$ \\
\hline BITP & 8DD & 76813.7 & 76226.6 & $250.7-240.7$ & 322.7 & 324.3 & 83.6 & 1" PvC & $08-29-90$ \\
\hline BTP & 9c & 74794.6 & 76203.0 & 109.9- 99.9 & 292.9 & 295.1 & 195.2 & 2" PVC & $10-03-90$ \\
\hline
\end{tabular}

(sories continued) 
BTP WELL SERIES (cont.)

\begin{tabular}{|c|c|c|c|c|c|c|c|c|c|}
\hline \multirow[b]{2}{*}{ Well D } & \multicolumn{2}{|c|}{ BRS Coordinates } & \multicolumn{3}{|c|}{ Elevations (ft MSL) } & \multirow{2}{*}{$\begin{array}{l}\text { Eff. Well } \\
\text { Depth (tt) }\end{array}$} & \multirow[b]{2}{*}{ Casing } & \multirow[b]{2}{*}{ Ingialled } & \multirow[b]{2}{*}{ Abandoned } \\
\hline & North & East & Screen Zone & Grnd. & Casing & & & & \\
\hline BTP 9D & 74805.3 & 76213.8 & $227.8-217.8$ & 292.8 & 294.9 & 77.1 & $2^{n}$ PVC & $10-03.90$ & \\
\hline BTP FDD & 74805.3 & 76213.8 & 245.8-235.7 & 292.8 & 294.9 & 59.2 & 1" PVC & 10.03 .90 & \\
\hline BTP 10C & 74641.9 & 74504.3 & 124.2-114.2 & 290.7 & 292.9 & 178.7 & 2" PVC & $09-05-90$ & \\
\hline BTP 10D & 74627.1 & 74504.1 & $235.6-225.6$ & 290.5 & 292.7 & 67.2 & 2" PVC $^{n}$ & $09-06-90$ & \\
\hline BTP 10DD & 74627.1 & 74604.1 & 255.5-245.5 & 290.5 & 292.7 & 47.2 & 1" PVC & $09-06-90$ & \\
\hline BTP 11C & 72922.7 & 76499.9 & 101.391 .3 & 291.3 & 293.4 & 202.1 & $2^{n}$ PVC & $11-16-90$ & \\
\hline IP 11D & 72983.6 & 76488.6 & $225.1-216.1$ & 291.1 & 293.4 & 78.3 & $2^{n}$ PVC & $11.12-90$ & \\
\hline WP 11DD & 72983.6 & 76488.6 & 243.1-293.0 & 291.1 & 293.4 & 60.4 & 1" PVC & $11-12-90$ & \\
\hline P $12 \mathrm{C}$ & 72289.2 & 74900.9 & $118.7-108.7$ & $\$ 08.7$ & 311.2 & 202.5 & $2^{n}$ PVC & $01-04-91$ & \\
\hline TP $12 D$ & 72281.1 & 74888.6 & $241.9-231.9$ & 307.9 & 310.4 & 78.5 & $2^{n}$ PVC & $01-04-91$ & \\
\hline BIP 12DD & 72281.1 & 74888.5 & 259.9-249.8 & $\$ 07.9$ & 310.4 & 60.6 & 1" PVC & $01.04-91$ & \\
\hline
\end{tabular}

Notes: The BIH Beries was known as the BIP Series until 1991 when the name was changed to avoid confusion with the characterization piezometers for the proposed Sanitary Landfill, Bite B, off E2 Road. Bee the Bit Beries.

The D and DD wells in this series are actually composed of two casings side by side in the same hole. The $D$ well is a $2^{n}$ casing referred to ae the lower casing. The DD well is a $1^{n}$ casing referred to as the upper casing. The upper casing does not extend as deeply in the hole as does the lower casing.

\section{CBR WELL SERIES}

Description: Central Shops Burning/Rubble Pit south of the Ford Building Seepage Basin Bldg. Number: 631-6G

Map: Fiqure A-21

\begin{tabular}{|c|c|c|c|c|c|c|c|c|c|}
\hline \multirow[b]{2}{*}{ Well ID } & \multicolumn{2}{|c|}{ BRS Coordinates } & \multicolumn{3}{|c|}{ Flevations (ft MSL) } & \multirow{2}{*}{$\begin{array}{l}\text { Eff. Well } \\
\text { Depth (ft) }\end{array}$} & \multirow[b]{2}{*}{ Casing } & \multirow[b]{2}{*}{ Ingtalled } & \multirow[b]{2}{*}{ Abandoned } \\
\hline & North & East & Bereen Zone & Grnd. & Caging & & & & \\
\hline $\begin{array}{ll}\text { CBR } & 1 D \\
\text { CBR } 2\end{array}$ & 60419.6 & 52822.1 & $260.9-290.9$ & 298.6 & $\$ 00.6$ & 69.7 & $\begin{array}{l}\text { 4" PVC }^{n} \text { PV PVC } \\
\text { 4" }\end{array}$ & $\begin{array}{l}02-16-90 \\
01-23-90\end{array}$ & \\
\hline $\begin{array}{ll}\text { CBR } & 2 D \\
\text { CBR } & \text { 3D }\end{array}$ & $\begin{array}{l}60368.9 \\
60388.5\end{array}$ & $\begin{array}{l}\text { E2694.0 } \\
52627.2\end{array}$ & $\begin{array}{l}253.8-233.8 \\
254.1-234.1\end{array}$ & $\begin{array}{l}298.8 \\
299.6\end{array}$ & $\begin{array}{l}300.9 \\
301.8\end{array}$ & $\begin{array}{l}67.1 \\
67.7\end{array}$ & $\begin{array}{l}4^{\prime \prime} \text { PVC } \\
4^{n} \text { PVC }\end{array}$ & $\begin{array}{l}02-16-90 \\
02-14-90\end{array}$ & \\
\hline
\end{tabular}

Notes: A note on the Monitoring Well Construction Diagram for CER 2 states that the well had grout contamination and would be abandoned. The well installation data mistakenly designated CER 2 as CER 20.

\section{CCB WELL SERIES}

Description: C-Area Coal Pile Runoff Containment Basin

Bldg. Number: 189-C

Map: Figure A-3

\begin{tabular}{|c|c|c|}
\hline \multirow[b]{2}{*}{ Well ID } & \multicolumn{2}{|c|}{ SRS Coordinates } \\
\hline & North & East \\
\hline & 65438.6 & 46990.1 \\
\hline В 2 & 65306.1 & 46893.6 \\
\hline CB 3 & 65187.5 & 47006.6 \\
\hline $\mathrm{CB} 4$ & 65310.2 & 47181.6 \\
\hline
\end{tabular}

Elevations (ft MSL)

Screen Zone Grnd. Casing

\begin{tabular}{lll}
$228.4-198.4$ & 276.4 & 278.6 \\
\hline
\end{tabular}

$\begin{array}{rrr}228.6-198.6 & 268.6 & 270.4\end{array}$

235.6-205.6 $265.6 \quad 267.4$

$\begin{array}{rrr}241.2-211.2 & 281.2 & 283.0\end{array}$
Iff. Well

Depth (ft) Casing Installed Abandoned

$80.2 \quad 4^{\prime \prime}$ PVC 09-15-81

$71.8 \quad 4^{n}$ PVC $09-21-31$

$61.8 \quad 4^{n}$ PVC 08-17.81

$71.8 \quad 4^{\prime \prime}$ PVC 08-16-81 
CCP WELL SERIES

Description: C-Area Coal Pile Study

Bldg. Number: Not applicable

Map: Figure A-3

\begin{tabular}{|c|c|c|c|c|c|c|c|c|c|}
\hline & SRS Coo & dinates & Elevations (f & MSL) & & Eff. Well & & & \\
\hline Well ID & North & Eagt & Screen Zono & Grnd. & Caping & Dopth (ft) & Casing & Ingtalled & Abandoned \\
\hline CCP 1A & 66659.9 & 46981.3 & 140.5-130.5 & 287.1 & 289.2 & 158.7 & $4^{n}$ PVC & $07-30-90$ & \\
\hline & & 46998.0 & 226.9-206.9 & 286.9 & 289.0 & 82.1 & & $08-09.90$ & \\
\hline
\end{tabular}

\section{CDB WELL SERIES}

Description: C-Area Disassembly Basin

Bldg. Number: 105-C

Map: Figure A-3

\begin{tabular}{|c|c|c|c|c|c|c|c|c|c|}
\hline & ERs Co & dinates & Plevations of & MBL) & & Eff. Well & & & \\
\hline Well ID & North & Past & Screen Zone & Grnd. & Coning & Depth (ft) & Caging & Installed & Abandoned \\
\hline $\begin{array}{l}\text { CDB } 1 \\
\text { CDB } 2\end{array}$ & $\begin{array}{l}67614.6 \\
67415.3\end{array}$ & $\begin{array}{l}46685.5 \\
46817.7\end{array}$ & $\begin{array}{l}216.6-196.7 \\
216.1-196.1\end{array}$ & $\begin{array}{l}286.6 \\
286.1\end{array}$ & $\begin{array}{l}288.9 \\
288.6\end{array}$ & $\begin{array}{l}93.2 \\
93.6\end{array}$ & $\begin{array}{l}4^{n} \text { PVC } \\
4^{\prime \prime} \text { PVC }\end{array}$ & $\begin{array}{l}08-01-86 \\
07-26-86\end{array}$ & \\
\hline
\end{tabular}

\section{CMP WELL SERIES}

Description: Chemicals, Metals, and Pesticides (CMP) Pits Bldg. Number: 080-17G, -17.1G, -18G, -18.1G, -18.2G, -18.3G, -19G Map: Figure A-22

\begin{tabular}{|c|c|c|c|c|c|c|c|c|c|}
\hline & SRS Coo & dinates & Mlevation (f & MBL) & & Brf. Well & & & \\
\hline Well ID & North & Sant & Ecreen Zone & Grnd. & Caning & Depth (t) & Casing & Installed & Abandone \\
\hline CMP 1 & 51692.6 & 53986.8 & $219.8-199.8$ & 310.4 & 312.9 & 113.1 & Bteel & $05.01-75$ & $0801-84$ \\
\hline CMP 2 & 51633.4 & 53957.8 & 206.9-202.9 & 311.0 & 313.6 & 110.6 & Stool & $06-01-76$ & $08.01-84$ \\
\hline CMP 3 & 51658.7 & 53921.6 & $208.0-186.0$ & 312.1 & $\$ 14.6$ & 128.6 & Steel & 0601.75 & $0801-84$ \\
\hline CMP & 51703.1 & 53848.5 & $204.4-184.4$ & 313.9 & 316.4 & 132.0 & Bteol & $05-01-76$ & $06-01-84$ \\
\hline CMP 6 & 61764.4 & 53768.6 & $220.0-200.0$ & $\$ 10.0$ & 312.0 & 112.0 & 4" Eteal & $09-16-78$ & $0801-84$ \\
\hline CMP 6 & 61811.9 & 58648.0 & 216.0-196.0 & 308.6 & 305.5 & 109.6 & 1" Eteol & $08-81-79$ & $06-01-84$ \\
\hline CMP 7 & 61673.2 & 63734.9 & $208.6-188.6$ & 317.9 & 319.9 & 131.3 & 4" Bteel & $09-1479$ & $08-01-84$ \\
\hline CMP 8 & 62881.4 & 64272.4 & 214.0 .184 .0 & 227.0 & 228.6 & 44.6 & 4" FVC & $08-11-82$ & \\
\hline CMPP $8 A$ & 62671.2 & 54270.2 & 23.6- 13.7 & 228.0 & 229.7 & 216.0 & $4^{\prime \prime}$ PVC & $07.01-84$ & \\
\hline CMP $8 B$ & 62674.6 & 64280.2 & $166.6-166.6$ & 227.7 & 229.6 & 72.9 & 4" PVC & $07-01-84$ & \\
\hline CMP 9 & 61673.9 & 63838.1 & 206.6-176.6 & 212.3 & 314.3 & 187.7 & $4^{\prime \prime}$ PVC & $06-26-82$ & $06-01-84$ \\
\hline CMP 9B & 81691.6 & 53842.3 & $169.0-149.0$ & 313.0 & 315.1 & 166.1 & $4^{n}$ PVC & $02-01-86$ & \\
\hline CMP 9C & 61684.9 & 53849.3 & 233.9-213.9 & 313.0 & 316.1 & 101.2 & $4^{\prime \prime}$ PVC & $02-01-86$ & \\
\hline CMP 10 & 61350.4 & 54006.5 & 218.8-188.8 & 308.8 & 310.9 & 122.1 & $4^{n}$ PVC & $08-20-82$ & \\
\hline CMP 10B & 61980.7 & 54005.9 & 147.4137 .4 & 308.0 & 310.8 & 179.4 & 4" PVC & $07-01-84$ & \\
\hline CMP 11 & 51481.3 & 53640.6 & 216.2-186.2 & 309.2 & 310.5 & 125.3 & $4^{\prime \prime}$ PVC & 08-23-82 & \\
\hline CMP 11B & 51466.6 & 53661.9 & 149.7-139.7 & 308.5 & 310.2 & 170.5 & $4^{*}$ PVC & $07-01-84$ & \\
\hline CMP 11TA & & & (168.6-178.6) & 308.0 & 310.8 & 489.1 & $10^{n} \mathrm{Cs}$ & & \\
\hline CMP 12 & B1956.4 & 53618.9 & $223.6-193.6$ & 281.6 & 282.9 & 89.3 & $4^{n} \mathrm{FVC}$ & $08-27-82$ & \\
\hline
\end{tabular}


CMP WELL SERIES (cont.)

\begin{tabular}{|c|c|c|c|c|c|c|c|c|c|}
\hline \multirow[b]{2}{*}{ Well ID } & \multicolumn{2}{|c|}{ SRS Ccordinates } & \multicolumn{3}{|c|}{ Elevations (ft MSL) } & \multirow{2}{*}{$\begin{array}{l}\text { Eff. Well } \\
\text { Depth (ft) }\end{array}$} & \multirow[b]{2}{*}{ Casing } & \multirow[b]{2}{*}{ Installed } & \multirow[b]{2}{*}{ Abandoned } \\
\hline & North & East & Screen Zone & Grnd. & Casing & & & & \\
\hline CMP 12A & 51949.2 & 53524.6 & 32.1- 22.1 & 281.9 & 284.1 & 262.0 & $4^{n}$ PVC & 07.01 .84 & \\
\hline CMP 12B & 51943.3 & 53517.7 & $158.0-148.0$ & 281.9 & 283.9 & 135.9 & $4^{n}$ PVC & $07.01-84$ & \\
\hline CMIP 13 & 61862.0 & 53945.5 & 212.7-182.7 & 287.7 & 289.2 & 106.5 & 4" PVC & $08-30-82$ & \\
\hline CMPP 13B & 51855.6 & 53937.8 & $144.2-134.2$ & 287.9 & 289.1 & 154.9 & $4^{n}$ PVC & $07-01.84$ & \\
\hline CMP 14B & 52376.4 & 52587.3 & 140.0 .130 .0 & 263.0 & 264.5 & 134.5 & $4^{\prime \prime}$ PVC & $07.01-84$ & \\
\hline CMP 14C & 52371.7 & 52579.6 & 215.9 .185 .1 & 262.8 & 264.1 & 79.0 & $4^{\prime \prime}$ PVC & $07-01-84$ & \\
\hline CMP 15A & 51357.2 & 52896.8 & 24.2- 14.2 & 274.7 & 276.5 & 262.3 & $4^{n} \mathrm{PVC}$ & 07.01 .84 & \\
\hline CMP 16B & 51349.6 & 52904.7 & $155.1 \cdot 145.1$ & 275.3 & 276.4 & 131.3 & $4^{n}$ PVC & $07-01-84$ & \\
\hline CMP 15C & 51361.4 & 62907.8 & $250.6-220.6$ & 275.5 & 277.0 & 56.4 & 4" PVC & $07-01-84$ & \\
\hline CMP $16 B$ & 51576.7 & 53049.9 & $151.7-141.7$ & 3158 & 317.7 & $\$ 76.0$ & $* *$ & 02.01 .85 & \\
\hline CMP 16C & 51569.5 & 53856.0 & 235.6-215.6 & 315.7 & 317.6 & 101.9 & ** & 02.01 .85 & \\
\hline
\end{tabular}

Notes: Twelve pumps were inctalled in the CMP wells in July 1984 (Geraghty \& Miller, 1985).

Inotallod dates for C.IP 1, 2, 3, and 4 are from Christensen and Gordon (1983). These dates are approximate. More definite dates are not given.

CuP 2 has a steel casing with a brase scree'a.

CL.P 9 was drilled 6-25-82 but was never sampled.

CMP IITA is a pinzomster well not monitored by IAPD/EMS.

CMIP 12 has been referred to incorrectly as C.MP 120.

CMP 13 has been referr ad to incorrectly as CM.P $13 C$.

The Screen Zone Elevation in parenthesis is in feet below MSL.

** MBB 168 and $16 \mathrm{C}$ have 4-in. PVC casing invide 16-in C8 casing. MBB 16 B has a 88 screen.

\section{COH WELL SERIES}

The COH Series was changed to the I.CO Series in 1985. This series is referred to as COH in Christensen and Gordon (1983). See the LCO Series.

\section{CRP WELL SERIES}

Description: C-Area Burning/Rubble Pit

Bldg. Number: 131-C

Map: Figure A-3

\begin{tabular}{|c|c|c|c|c|c|c|c|c|c|}
\hline \multirow[b]{2}{*}{ Well ID } & \multicolumn{2}{|c|}{ SRS Coordinates } & \multicolumn{3}{|c|}{ Elevations (ft MSL) } & \multirow{2}{*}{$\begin{array}{l}\text { Dff. Well } \\
\text { Depth (ft) }\end{array}$} & \multirow[b]{2}{*}{ Casing } & \multirow[b]{2}{*}{ Installed } & \multirow[b]{2}{*}{ Abandonod } \\
\hline & North & East & Bcreen Zone & Grnd. & Casing & & & & \\
\hline $\begin{array}{ll}\text { CRP } 1 \\
\text { CRP } 2\end{array}$ & $\begin{array}{l}68617.7 \\
69043.0\end{array}$ & $\begin{array}{l}44372.2 \\
44336.4\end{array}$ & $\begin{array}{l}217.8 \cdot 187.8 \\
201.8 .171 .8\end{array}$ & $\begin{array}{l}272.8 \\
276.8\end{array}$ & $\begin{array}{l}274.6 \\
278.7\end{array}$ & $\begin{array}{r}86.8 \\
106.9\end{array}$ & $\begin{array}{l}4^{n} \text { PVC } \\
4^{n} \text { PVC }\end{array}$ & $\begin{array}{l}09-08-83 \\
09.16-83\end{array}$ & \\
\hline $\begin{array}{ll}\text { CRP } & 3 \\
\text { CRP } & 4\end{array}$ & $\begin{array}{l}68666.6 \\
68447.4\end{array}$ & $\begin{array}{l}44001.0 \\
44101.2\end{array}$ & $\begin{array}{l}214.0-184.0 \\
210.7-180.7\end{array}$ & $\begin{array}{l}264.0 \\
265.7\end{array}$ & $\begin{array}{l}265.8 \\
267.7\end{array}$ & $\begin{array}{l}81.8 \\
87.0\end{array}$ & $\begin{array}{l}\text { 4" PVC } \\
\text { 4" PVC }\end{array}$ & $\begin{array}{l}09-13-89 \\
06-28-84\end{array}$ & \\
\hline
\end{tabular}


CSA WELL SERIES

Description: Hydrofluoric Acid Spill Area

Bldg. Number: 631-4G

Map: Figure A-21

\begin{tabular}{|c|c|c|c|c|c|c|c|c|c|}
\hline \multirow[b]{2}{*}{ Well ID } & \multicolumn{2}{|c|}{ 8Rs Coordinates } & \multicolumn{3}{|c|}{ Elevations (A MSL) } & \multirow{2}{*}{$\begin{array}{l}\text { Bif. Well } \\
\text { Depth (f) }\end{array}$} & \multirow[b]{2}{*}{ Casing } & \multirow[b]{2}{*}{ Installed } & \multirow[b]{2}{*}{ Abandoned } \\
\hline & Norte & Fant & Ecreen Zone & Grnd. & Caning & & & & \\
\hline CBA & 61808.4 & 60197.0 & $262.0-232.0$ & 289.0 & 290.8 & 68.8 & $4^{*}$ PVC & $06-06-84$ & \\
\hline $\operatorname{CBA} 2$ & 61761.8 & 60218.6 & 248.2-218.2 & 288.2 & 290.1 & 71.9 & 4" PVC & $06-08-84$ & \\
\hline CBA 3 & 61720.2 & 50173.2 & $248.6-218.6$ & 287.6 & 289.4 & 70.8 & $4^{n}$ PVC & 06.07 .84 & \\
\hline $\operatorname{CsA} 4$ & 61781.9 & 50132.7 & $248.4-218.4$ & 288.4 & 290.4 & 72.0 & 4" PVC & $06-08-84$ & \\
\hline
\end{tabular}

\section{CSB WELL SERIES}

Description: C-Area Reactor Seepage Basins

Bldg. Number: 904-66G, -67G, -68G

Map: Figure A-3

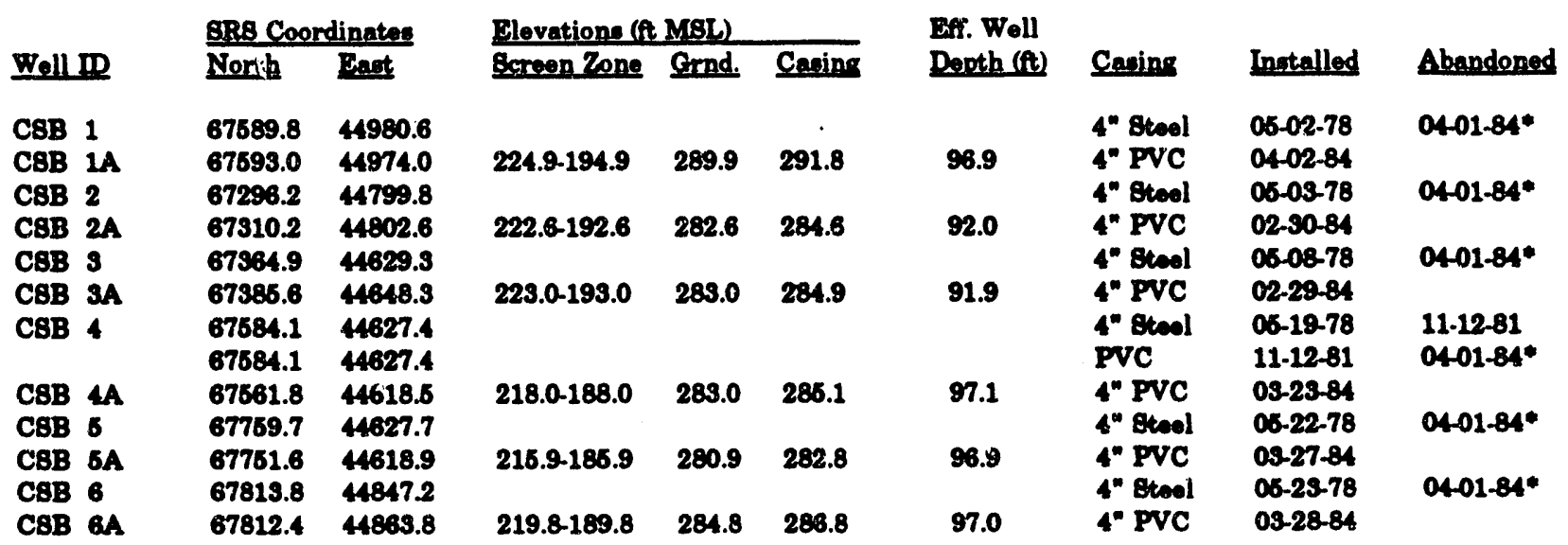

Noten: CSBB 4 was redrilled 11-12-81, and the ctoel casing was replaced with PVC.

\section{CSD WELL SERIES}

Description: Central Shops Diesel Spill Characterization and Remediation Wells Bldg. Number: 8318

Map: Figure A-21

\begin{tabular}{|c|c|c|c|c|c|c|c|c|}
\hline \multirow[b]{2}{*}{ Well D } & \multicolumn{2}{|c|}{ BR8 Coordinates } & \multicolumn{3}{|c|}{ Elevations (A MSL) } & \multirow{2}{*}{$\begin{array}{l}\text { Efr. Woll } \\
\text { Depth (A) }\end{array}$} & \multirow[b]{2}{*}{ Caring } & \multirow[b]{2}{*}{ Installed } \\
\hline & North & Fast & Ecreen Zone & Grnd. & Casing & & & \\
\hline BD ID & 63255.8 & 50170.5 & $273.4-238.4$ & 313.4 & 316.4 & 77.0 & $4^{n} \mathrm{P}$ & $01-10-89$ \\
\hline BD 2D & 63126.2 & 60144.0 & $258.8-233.8$ & 308.8 & 310.8 & 77.0 & 4" PVC & $01.11-89$ \\
\hline C8D $4 D$ & 63143.8 & 60058.9 & 263.6-213.5 & 306.6 & 308.6 & $96.0^{\circ}$ & $4^{*}$ PVC & $01-11-89$ \\
\hline CSD $8 D$ & 63195.0 & 49903.1 & 256.8-226.8 & 301.8 & 303.9 & 77.1 & $4^{\circ}$ PVC & $01-20-89$ \\
\hline
\end{tabular}

(eries continued) 
(3)

CSD WELL SERIES (cont.)

\begin{tabular}{|c|c|c|c|c|c|c|c|c|c|}
\hline \multirow[b]{2}{*}{ Well ID } & \multicolumn{2}{|c|}{ SRS Coordinates } & \multicolumn{3}{|c|}{ Elevations (A MSL) } & \multirow{2}{*}{$\begin{array}{l}\text { Eff. Well } \\
\text { Depth (ft) }\end{array}$} & \multirow[b]{2}{*}{ Casing } & \multirow[b]{2}{*}{ Installed } & \multirow[b]{2}{*}{ Abandoned } \\
\hline & North & East & Screen Zone & Grnd. & Casing & & & & \\
\hline Con 9D & 63080.9 & 49838.8 & 256.2-226.2 & 296.2 & 298.2 & 72.0 & 4" PVC & 01-18-89 & \\
\hline C8D 10D & 63094.1 & 49806.5 & $254.5-224.5$ & 294.6 & 296.6 & 72.1 & 4" PVC & $01-13-89$ & \\
\hline CSD 11D & 63956.3 & 49763.9 & $250.9-220.9$ & 290.9 & 293.0 & 72.1 & $4^{n}$ PVC & $01-20-89$ & \\
\hline CSD 12D & 63004.7 & 49937.3 & $254.6-224.6$ & 299.5 & 301.6 & 77.1 & 4" PVC & $01-12.89$ & \\
\hline CSD 13D & 62897.8 & 49665.5 & $252.4-202.4$ & 287.4 & 289.6 & 87.1 & 6" PVC & $01-24-89$ & \\
\hline
\end{tabular}

\section{CSH WELL SERIES}

The CSH Series was changed to the HWS Series in 1985. This series is also referred to as CTS in Christensen and Gordon (1983). See the HWS Series.

\section{CSO WELL SERIES}

Description: Fire Department Training Facility

Bldg. Number: 904-113G

Map: Figure A-21

\begin{tabular}{|c|c|c|c|c|c|c|c|c|c|}
\hline \multirow[b]{2}{*}{ Well ID } & \multicolumn{2}{|c|}{ gRs Coordinates } & \multicolumn{3}{|c|}{ Plevatione (A MSL) } & \multirow{2}{*}{$\begin{array}{l}\text { Eff. Well } \\
\text { Depth (ft) }\end{array}$} & \multirow[b]{2}{*}{ Casing } & \multirow[b]{2}{*}{ Intalled } & \multirow[b]{2}{*}{ Abandoned } \\
\hline & North & East & Bcreen Zone & Grnd. & Casing & & & & \\
\hline $\begin{array}{ll}\text { CsO } 1 \\
\text { CsO } 2\end{array}$ & $\begin{array}{l}61071.1 \\
61114.9\end{array}$ & $\begin{array}{l}52484.2 \\
52559.0\end{array}$ & $\begin{array}{l}262.0-232.0 \\
239.7-209.7\end{array}$ & $\begin{array}{l}302.0 \\
299.7\end{array}$ & $\begin{array}{l}303.9 \\
301.9\end{array}$ & $\begin{array}{l}71.9 \\
92.2\end{array}$ & $\begin{array}{l}4^{\prime \prime} \text { PVC } \\
4^{\prime \prime} \text { PVC }\end{array}$ & $\begin{array}{l}07-05-83 \\
06-13-84\end{array}$ & \\
\hline
\end{tabular}

\section{CSR WELL SERIES}

Description: Central Shops Burning/Rubble Pits Bldg. Number: 631-1G, $-5 \mathrm{G},-6 \mathrm{G}$

Map: Figure A-21

\begin{tabular}{|c|c|c|c|c|c|c|c|c|c|}
\hline \multirow[b]{2}{*}{ Well ID } & \multicolumn{2}{|c|}{ SRS Coordinates } & \multicolumn{3}{|c|}{ Elevations (ft MSL) } & \multirow{2}{*}{$\begin{array}{l}\text { Eff. Well } \\
\text { Depth (ft) }\end{array}$} & \multirow[b]{2}{*}{ Casing } & \multirow[b]{2}{*}{ Installed } & \multirow[b]{2}{*}{ Abandoned } \\
\hline & North & East & Bcreen Zone & Grnd. & Casing & & & & \\
\hline CSR & 64413.1 & 52804.3 & 267.2-237.2 & 272.2 & 274.1 & 36.9 & $4^{\prime \prime}$ PVC & $07-12-83$ & \\
\hline CER & 64733.2 & 53525.1 & 285.6-256.5 & 295.5 & 297.7 & 42.2 & $4^{n}$ PVC & $07-14-83$ & \\
\hline CBR & 65234.8 & 53229.9 & 268.1-238.1 & 289.1 & 285.2 & 47.1 & $4^{n}$ PVC & $07.12-83$ & \\
\hline CSR 4 & 64412.8 & 53214.4 & $267.6-237.6$ & 282.6 & 284.7 & 47.1. & $4^{n} \mathrm{PVC}$ & $06-16-84$ & \\
\hline
\end{tabular}

Notes: The wells are located around pits 631.1G and 631.5G.

\section{CTS WELL SERIES}

This series, referred to as CTS in Christensen and Gordon (1983), was changed to the HWS Series in 1985. In some reports of the raw data for the HWS Series, the wells are designated CSH. See the HWS Series. 
DBP WELL SERIES

Description: D-Area Burning/Rubble Pits

Bldg. Number: 431-D, -1D

Map: Figure A-20

\begin{tabular}{|c|c|c|c|c|c|c|c|c|c|}
\hline \multirow[b]{2}{*}{ Well ID } & \multicolumn{2}{|c|}{ BRS Coordinates } & \multicolumn{3}{|c|}{ Elevations (ft MSL) } & \multirow{2}{*}{$\begin{array}{l}\text { Eff. Well } \\
\text { Depth (ft) }\end{array}$} & \multirow[b]{2}{*}{ Casing } & \multirow[b]{2}{*}{ Installed } & \multirow[b]{2}{*}{ Abandoned } \\
\hline & North & East & Screen Zone & Grnd. & Casing & & & & \\
\hline DBP 1 & 66691.4 & 18661.8 & 123.2-93.2 & 133.2 & 135.2 & 42.0 & $4^{N}$ PVC & $09-20-83$ & \\
\hline DBP 2 & 66478.2 & 18407.3 & $114.9-84.3$ & 124.3 & 126.3 & 42.0 & $4^{\prime \prime}$ PVC & $09-19-83$ & \\
\hline DBP 9 & 66775.5 & 18427.6 & $116.4-86.4$ & 126.4 & 128.3 & 41.9 & $4^{\prime \prime}$ PVC & 09.21 .89 & \\
\hline DBP 4 & 66679.6 & 18342.1 & $114.2-84.2$ & 124.2 & 126.2 & 42.0 & 4" PVC & $06-25-84$ & \\
\hline
\end{tabular}

\section{DCB WELL SERIES}

Description: D-Area Coal Pile Runoff Containment Basin and Ash Basins (DCB 11, 12, 13, 14, 15, and 16 are located around the Ash Basin in D Area)

Bldg. Number: 489-D

Map: Figure A.20

\begin{tabular}{|c|c|c|c|c|c|c|c|c|c|}
\hline \multirow[b]{2}{*}{ Well ID } & \multicolumn{2}{|c|}{ SRs Coordinates } & \multicolumn{3}{|c|}{ Plovations (ft MSL) } & \multirow{2}{*}{$\begin{array}{l}\text { Bff. Well } \\
\text { Depth (ft) }\end{array}$} & \multirow[b]{2}{*}{ Caring } & \multirow[b]{2}{*}{ Inotalled } & \multirow[b]{2}{*}{ Abandoned } \\
\hline & North & East & Bcreen Zone & Grnd. & Casing & & & & \\
\hline $\begin{array}{l}\text { DCB } 1 \\
\text { DCB } 1 A\end{array}$ & $\begin{array}{l}64033.9 \\
64028.5\end{array}$ & $\begin{array}{l}19862.3 \\
19866.3\end{array}$ & $\begin{array}{l}122.1-102.1 \\
120.1 \cdot 90.1\end{array}$ & $\begin{array}{l}124.9 \\
126.1\end{array}$ & $\begin{array}{l}126.9 \\
127.1\end{array}$ & $\begin{array}{l}24.8 \\
37.0\end{array}$ & $\begin{array}{l}4^{n} \text { Stool } \\
4^{n} \text { PVC }\end{array}$ & $\begin{array}{l}04-21-80 \\
03-08-84\end{array}$ & $03-09-84$ \\
\hline $\begin{array}{l}\text { DCB } 2 \\
\text { DCB } 2 A\end{array}$ & $\begin{array}{l}63431.5 \\
63438.1\end{array}$ & $\begin{array}{l}20902.6 \\
20895.2\end{array}$ & $\begin{array}{l}128.9-108.9 \\
127.4-97.4\end{array}$ & $\begin{array}{l}131.9 \\
132.4\end{array}$ & $\begin{array}{l}139.9 \\
134.3\end{array}$ & $\begin{array}{l}25.0 \\
36.9\end{array}$ & $\begin{array}{l}4^{n} \text { Stoel } \\
4^{n} \text { PVC }\end{array}$ & $\begin{array}{l}04-18-80 \\
03-07-84\end{array}$ & $03-09-84$ \\
\hline $\begin{array}{ll}\text { DCB } & 3 \\
\text { DCB } & 3 A\end{array}$ & $\begin{array}{l}62697.9 \\
62674.9\end{array}$ & $\begin{array}{l}20906.5 \\
20899.9\end{array}$ & $\begin{array}{l}127.9-107.9 \\
126.2-96.2\end{array}$ & $\begin{array}{l}132.2 \\
131.2\end{array}$ & $\begin{array}{l}134.2 \\
133.0\end{array}$ & $\begin{array}{l}26.3 \\
36.8\end{array}$ & $\begin{array}{l}4^{\prime \prime} \text { Bteol } \\
4^{\prime \prime} \text { PVC }\end{array}$ & $\begin{array}{l}04-18-80 \\
09-09-84\end{array}$ & $03-09-84$ \\
\hline $\begin{array}{l}\text { DCB } \\
\text { DCB }\end{array}$ & $\begin{array}{l}62688.2 \\
62678.8\end{array}$ & $\begin{array}{l}20486.2 \\
20493.8\end{array}$ & $\begin{array}{l}126.6-106.6 \\
122.6-92.6\end{array}$ & $\begin{array}{l}127.8 \\
127.6\end{array}$ & $\begin{array}{l}129.8 \\
129.6\end{array}$ & $\begin{array}{l}23.2 \\
37.0\end{array}$ & $\begin{array}{l}4^{n} \text { Stoel } \\
4^{n} \text { PVC }\end{array}$ & $\begin{array}{l}04-22-80 \\
03-07-84\end{array}$ & $09-09-84$ \\
\hline $\begin{array}{ll}\text { DCB } & 5 \\
\text { DCB } & 5 \mathrm{~A}\end{array}$ & 63112.9 & 20147.6 & 119.9-104.5 & 121.3 & 123.3 & 18.8 & 4" Btoel & 04-21-80 & $08-09-84$ \\
\hline DCB 6 & 64167.9 & 19979.3 & $129.6-109.5$ & $\begin{array}{l}120.9 \\
191.6\end{array}$ & $\begin{array}{l}122.9 \\
139.2\end{array}$ & $\begin{array}{l}37.0 \\
28.7\end{array}$ & $\begin{array}{l}\text { 4" PVC } \\
\text { 4" PVC }\end{array}$ & $\begin{array}{l}03-08-84 \\
08-31-87\end{array}$ & \\
\hline DCB 7 & 64001.4 & 20036.3 & 128.9-108.9 & 130.9 & 132.8 & 23.9 & 4" PVC & $08-31-87$ & \\
\hline DCB 8 & 63473.9 & 21014.1 & $130.9-110.3$ & 134.8 & 136.8 & 26.5 & 4" PVC & $08-31-87$ & \\
\hline DCB 9 & 64190.6 & 19807.4 & $117.3-97.3$ & 120.2 & 122.3 & 26.0 & $4^{*}$ PVC & $08-31-87$ & \\
\hline DCB 10 & 63803.1 & 19862.3 & 119.8. 99.8 & 121.8 & 123.9 & 24.1 & $4^{n}$ PVC & $08-31.87$ & \\
\hline DCB 11 & 64638.3 & 19248.6 & $126.8-106.8$ & 128.8 & 180.6 & 23.8 & $4^{n}$ PVC & $08-81.87$ & \\
\hline DCB 12 & 65160.0 & 18629.8 & 112.0 92.0 & 116.0 & 116.9 & 24.9 & 4" PVC & $08-31-87$ & \\
\hline DCB 13 & 63842.6 & 19235.4 & 122.1-102.0 & 127.8 & 129.8 & 27.8 & $4^{\prime \prime}$ PVC & $06.30-88$ & \\
\hline DCB 14 & 64909.8 & 19392.4 & 114.6- 94.6 & 127.6 & 129.4 & 34.8 & 4" PVC & $08-26-88$ & $08-29-90$ \\
\hline DCB 16 & 64607.4 & 17636.9 & 119.9-99.8 & 125.4 & 127.6 & 27.8 & $4^{\prime \prime}$ PVC & $06-29-88$ & \\
\hline DCB 16 & 63966.0 & 17611.2 & $120.1-100.1$ & 125.9 & 127.9 & 27.8 & $4^{n}$ PVC & $06-28-88$ & \\
\hline
\end{tabular}




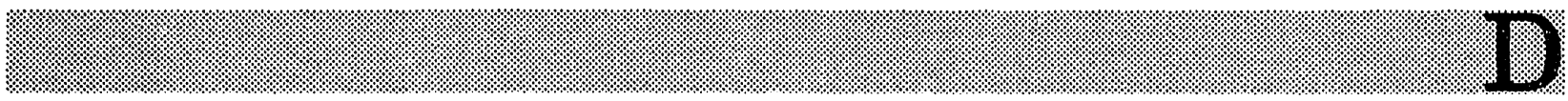

\section{DOB WELL SERIES}

Description: D-Area Oil Disposal Basin

Bldg. Number: 631-G

Map: Figure A-20

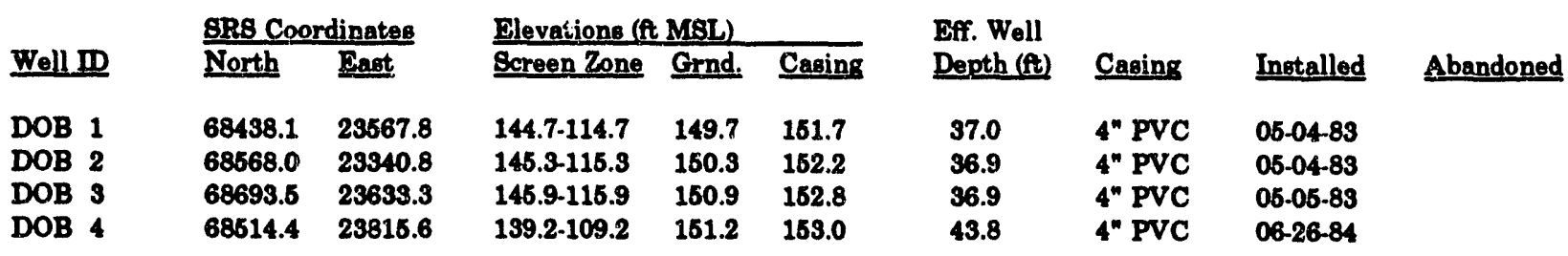

\section{DRB WELL SERIES}

Description: Deep Rock Borings

Bldg. Number: Not applicable

Map: See Figures A-2, A.8, A-9, A-11, A-12, and A-14

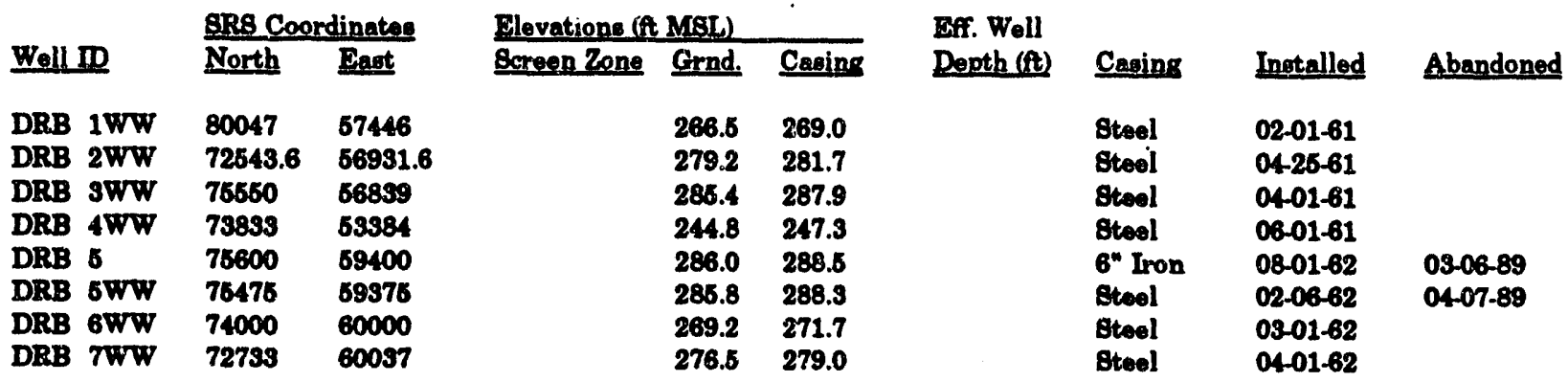

Notes: The Well Abandonment Report for DRB 6 reports that this well did not have a screen.

DRB 6 WW was inotalled as a tomporary production well to provide water for Deep Rock Boring 6 (DRB 6).

\section{DWP WELL SERIES}

The DWP Series is now known as the BH Series. See the BH Series. 


\section{F WELL SERIES}

Description: F-Area Seepage Basins

Bldg. Number: 904-41G, -42G, -43G

Map: Figures A-2, A-12, and A-13

\begin{tabular}{|c|c|c|c|c|c|c|c|c|c|}
\hline \multirow{2}{*}{ Well ID } & \multicolumn{2}{|c|}{ BRS Coordinater } & \multicolumn{3}{|c|}{ Plevatione (ft MSL) } & \multirow{2}{*}{$\begin{array}{l}\text { Eff. Well } \\
\text { Depth (A) }\end{array}$} & \multirow[b]{2}{*}{ Casing } & \multirow[b]{2}{*}{ Inotalled } & \multirow[b]{2}{*}{ Abandoner } \\
\hline & Nortt & East & Bcreen Zone & Grnd. & Caring & & & & \\
\hline 1 & 76604.7 & 51302.8 & 266.9-251.9 & 281.4 & 281.9 & $\mathbf{3 0 . 0}$ & $\mathbf{S}^{\infty}$ Steel & $03-06-66$ & $05-10-89$ \\
\hline 2 & 76677.4 & 51484.3 & $217.0-207.0$ & 280.2 & 281.4 & 74.4 & 3" Steel & $01-26-66$ & \\
\hline 3 & 75655.6 & 51494.0 & 217.1.207.1 & 279.2 & 279.6 & 72.6 & $9^{n}$ Stoel & $01-27-66$ & $10-01-77^{*}$ \\
\hline 4 & 75614.1 & 51810.1 & 217.8-207.8 & 279.6 & 280.1 & 72.3 & $3^{n}$ Steol & $01.14-66$ & $09-20-78$ \\
\hline 6 & 75646.7 & 61464.3 & $263.0-268.0$ & 279.0 & 279.6 & 21.6 & $3^{n}$ Bteel & $01-17-66$ & $01-01-60^{*}$ \\
\hline 6 & 76489.4 & 61144.0 & $274.4-264.4$ & 277.4 & 278.1 & 13.7 & 3n Bteel & $03-06-66$ & $01-01-60 *$ \\
\hline 7 & 75450.6 & 51168.8 & 272.9-262.9 & 276.9 & 277.6 & 14.7 & $\mathbf{S}^{n} \mathrm{Al}$ & $03-07-66$ & $03-28-89$ \\
\hline 8 & 75206.4 & 50948.1 & 215.4-205.4 & 277.1 & 277.7 & 72.3 & $3^{n}$ Steel & $01-05-66$ & $04-11-89$ \\
\hline 9 & 76016.1 & 50651.3 & 213.1-203.1 & 268.3 & 269.3 & 66.2 & $3^{n}$ Stoel & $01-09-66$ & \\
\hline 510 & 75156.3 & 50444.3 & 276.6-266.6 & 277.5 & 280.1 & 13.6 & $3^{n}$ Steel & $03-05-66$ & \\
\hline F 11 & 75144 & 60438 & $214.3-204.3$ & 277.6 & 280.8 & 76.5 & S" Stoel & $02-07-66$ & $01.01-60^{*}$ \\
\hline F 12 & 74895 & 60217 & 272.4-262.4 & 279.4 & 280.1 & 17.7 & $3^{n}$ Steel & $03-06-66$ & $01-01-60$ \\
\hline 13 & 74870 & 50201 & 271.8-261.8 & 278.8 & 279.6 & 17.7 & 3" Steel & $08-05-66$ & $01-0160^{*}$ \\
\hline 14 & 74807.1 & 50010.9 & & 278.2 & 276.7 & & Bteel & $08-01-62$ & \\
\hline 15 & 74716.0 & 50738.3 & & 251.6 & 253.6 & & Steel & $03-01-62$ & \\
\hline 16 & 74697.1 & 50029.0 & & 252.9 & 255.9 & & Bteol & $08-01-62$ & \\
\hline 17 & 74308.4 & 50468.4 & & 237.0 & 239.8 & & Bteol & $03-01-62$ & \\
\hline 18 & 74170.2 & 60108.0 & & 234.6 & 237.1 & & Btool & $08-01-62$ & $08-16-86$ \\
\hline $18 A$ & 74170.2 & 60108.0 & $204.4-194.4$ & 231.4 & 233.4 & $\mathbf{3 9 . 0}$ & 4" PVC & $01-16-86$ & \\
\hline 19 & 74245.9 & 49672.9 & & 241.4 & 243.9 & & Btoel & $08-01-62$ & \\
\hline 20 & 75227 & 48967 & & 279.2 & 281.7 & & Bteel & $03-23-62$ & $0401-85^{*}$ \\
\hline 21 & 75242 & 48121 & & 279.6 & 282.0 & & Btoel & $08-23-63$ & $04-01-88^{\circ}$ \\
\hline 22 & 75613 & 47470 & & 280.9 & 282.8 & & Steel & $09-29-62$ & $0401-86 *$ \\
\hline 23 & 75460.1 & 50081.1 & & 279.7 & 282.2 & & 1" Bteel & $08-01-67$ & $08-21-89$ \\
\hline 24 & 76069.0 & 51322.9 & & 290.2 & 291.0 & & Steel & $0301-62$ & \\
\hline 25 & 75083.8 & 51322.1 & & 269.7 & 260.9 & & Steel & $08-01-62$ & \\
\hline 26 & 75678 & 60269 & & 285.7 & 288.2 & & Bteel & & \\
\hline 27 & 75648 & 60087 & & 283.9 & 286.4 & & Stoel & & \\
\hline 28 & 76319 & 49928 & & 283.8 & 286.3 & & Steol & & \\
\hline 29 & 75141 & 49813 & & 283.2 & 286.7 & & Steel & & \\
\hline 30 & 74995 & 49697 & & 282.0 & 284.6 & & Stoel & & \\
\hline 31 & 75419 & 49813 & & 282.1 & 284.6 & & Steel & & \\
\hline 32 & & & & 291.7 & 294.2 & & Stoel & $01-01-67^{*}$ & $10-01.60 *$ \\
\hline 33 & 75113 & 49594 & & 289.0 & 291.6 & & Steel & $04-01.67 *$ & $10-01-60^{*}$ \\
\hline 34 & 74986 & 49962 & & 280.2 & 282.7 & & S" Al & & $03-27-89$ \\
\hline
\end{tabular}

Notes: According to the Drilling Roquest for P 18A, the well was drilled in the eame hole as $\mathbf{P} 18$.

The Well Abandonment Report for $\mathbf{P} 25$ reports that this well did not have a screen. 


\section{FAC WELL SERIES}

Description: F-Area Acid/Caustic Basin

Bldg. Number: 904-74G

Map: Figure A-12

\begin{tabular}{|c|c|c|c|c|c|c|c|c|c|}
\hline \multirow[b]{2}{*}{ Well I } & \multicolumn{2}{|c|}{ ERS Coordinates } & \multicolumn{3}{|c|}{ Blevations (At MSL) } & \multirow{2}{*}{$\begin{array}{l}\text { Eff. Well } \\
\text { Depth (tt) }\end{array}$} & \multirow[b]{2}{*}{ Casing } & \multirow[b]{2}{*}{ Installed } & \multirow[b]{2}{*}{ Abandoned } \\
\hline & North & Fagt & Bcreen Zone & Grnd. & Casing & & & & \\
\hline FAC 1 & 78169.6 & 55304.9 & $256.2-225.2$ & 310.2 & 312.2 & 87.0 & $4^{n}$ PVC & $08-18-83$ & $03-10-89$ \\
\hline & 78044.1 & 65244.4 & $266.8-226.8$ & 311.8 & 313.7 & 86.9 & $4^{n}$ PVC & $08-24-83$ & $09-10-89$ \\
\hline FAC 3 & 78018.3 & 65322.7 & $254.8-224.8$ & 909.8 & 311.8 & 87.0 & $4^{n}$ PVC & $08-26-89$ & \\
\hline & 78223.8 & 65472.9 & $237.8-207.8$ & 307.8 & 309.9 & 102.1 & $4^{n}$ PVC & $07-20-84$ & \\
\hline & 77960.3 & 55241.3 & $294.0-214.0$ & $\mathbf{3 1 4 . 0}$ & 316.8 & 101.8 & $4^{n}$ PVC & $09-02-88$ & \\
\hline FAC & 78129.0 & 56336.5 & 236.2-216.2 & 310.8 & 312.6 & 96.3 & 4" PVC & $09-15-88$ & \\
\hline & 78123.4 & 56956.2 & 2s5.7-215.7 & 310.3 & 312.0 & 96.3 & 4" PVC & $09-16-88$ & \\
\hline FAC 8 & 78090.9 & 85366.0 & $296.0-216.0$ & 309.0 & 311.0 & 95.0 & $4^{n}$ PVC & $09-09-88$ & \\
\hline
\end{tabular}

Notes: PAC 2 was redrilled when the first hole collapeed on 8-22-89 because of water from the basin.

The firet borehole for FAC 7 was abandoned 9-09-88 because a wrench was dropped in the borehole.

\section{FAL WELL SERIES}

Description: F-Area A Line

Bldg. Number: 221-1F

Map: Figure A-12

\begin{tabular}{|c|c|c|c|c|c|c|c|c|}
\hline & SRS Coordinates & Blevations of & MSL) & & Enff. Well & & & \\
\hline Well ID & North Eagt & Sereen Zone & Grnd. & Cogin- & Depth (f) & Caning & Inetalled & Abandoned \\
\hline $\begin{array}{l}\text { FAL } 1 \\
\text { FAL } 2\end{array}$ & $\begin{array}{ll}78115.9 & 63766.4 \\
78231.9 & 63757.4\end{array}$ & $\begin{array}{l}238.6-207.0 \\
238.0-206.6\end{array}$ & $\begin{array}{l}310.5 \\
310.0\end{array}$ & $\begin{array}{l}312.9 \\
312.1\end{array}$ & $\begin{array}{l}105.9 \\
105.6\end{array}$ & $\begin{array}{l}\text { 4" PVC } \\
4^{*} \text { PVC }\end{array}$ & $\begin{array}{l}03-03-88 \\
03-07-86\end{array}$ & \\
\hline
\end{tabular}

\section{FBP WELL SERIES}

Description: F-Area Burning/Rubble Pits

Bldg. Number: 231-F, -1F

Map: Figure A-12

\begin{tabular}{|c|c|c|c|c|c|c|c|c|c|}
\hline & \multicolumn{2}{|c|}{ gRs Coordinates } & \multicolumn{3}{|c|}{ Plevations (ft MSL) } & \multirow{2}{*}{$\begin{array}{l}\text { Bfi. Well } \\
\text { Depth (ft) }\end{array}$} & \multirow[b]{2}{*}{ Casing } & \multirow[b]{2}{*}{ Installed } & \multirow[b]{2}{*}{ Abandoned } \\
\hline Well ID & North & Fast & Bcreen Zone & Grnd. & Casing & & & & \\
\hline $\begin{array}{ll}\text { FBP } & 1 \\
\text { FBP } & 1 A\end{array}$ & $\begin{array}{l}78887.6 \\
78893.0\end{array}$ & $\begin{array}{l}51116.7 \\
51080.7\end{array}$ & $\begin{array}{l}207.3-177.3 \\
191.8161 .8\end{array}$ & $\begin{array}{l}282.3 \\
285.8\end{array}$ & 285.9 & 108.6 & & 09-06-83 & 06.18 .84 \\
\hline $\begin{array}{ll}\text { FBP } 2 \\
\text { FBP } 2 A\end{array}$ & $\begin{array}{l}79668.4 \\
79711.4\end{array}$ & $\begin{array}{l}50556.9 \\
50534.1\end{array}$ & $\begin{array}{l}213.1-183.1 \\
167.1-137.1\end{array}$ & $\begin{array}{l}288.1 \\
287.1\end{array}$ & $\begin{array}{l}290.1 \\
289.1\end{array}$ & $\begin{array}{l}107.0 \\
162.0\end{array}$ & $\begin{array}{l}\text { 4" PVC } \\
\text { 4" PVC }\end{array}$ & $\begin{array}{l}08-81-83 \\
06-17-84\end{array}$ & $04-01-84^{*}$ \\
\hline $\begin{array}{ll}\text { FBP } & 3 \\
\text { FBP } & 3 A \\
\text { FBP } & 4\end{array}$ & $\begin{array}{l}79841.1 \\
79838.9 \\
79320.0\end{array}$ & $\begin{array}{l}50927.7 \\
50913.4 \\
51368.2\end{array}$ & $\begin{array}{l}215.2-185.2 \\
171.0-141.0 \\
195.2 .165 .2\end{array}$ & $\begin{array}{l}290.2 \\
291.0 \\
284.2\end{array}$ & $\begin{array}{l}290.2 \\
292.9 \\
286.3\end{array}$ & $\begin{array}{l}105.0 \\
151.9 \\
121.1\end{array}$ & $\begin{array}{l}\text { 4" PVC } \\
\text { 4" PVC } \\
\text { 4" PVC }\end{array}$ & $\begin{array}{l}09-02-89 \\
05-15-84 \\
07.19-84\end{array}$ & $04-01-84^{*}$ \\
\hline
\end{tabular}

Notes: FBP 2A was first drilled 4-16-84 according to the Field Activities Log. A note indicates that although the well had $26 \mathrm{ft}$ of water in the ecreen zone, it would not produce when blown with air. Consequently, the well was redrilled 6-17-84. 


\section{FC WELL SERIES}

Description: F-Area Hydrology Cluster

Bldg. Number: Not applicable

Map: Figures A-1, A-2, A-11, and A-12

\begin{tabular}{|c|c|c|c|c|c|c|c|c|}
\hline \multirow{2}{*}{ Well ID } & \multicolumn{2}{|c|}{ gRS Coordinates } & \multicolumn{3}{|c|}{ Flevatione (ft MSL) } & \multirow{2}{*}{$\begin{array}{l}\text { Dff. Well } \\
\text { Depth (ft) }\end{array}$} & \multirow[b]{2}{*}{ Coging } & \multirow[b]{2}{*}{ Installed } \\
\hline & North & Esast & Bcreen Zone & Grond. & Casing & & & \\
\hline IA & 79664.5 & 63116.1 & 101.7. 96.7 & 316.7 & 318.6 & 221.9 & $4.19^{n}$ Oteol & $07.29-76$ \\
\hline 1B & 79672.4 & 63116.0 & $156.8-161.8$ & 316.8 & 318.6 & 166.7 & 4.13" Stoel & $08-04-76$ \\
\hline $1 \mathrm{C}$ & 79680.1 & 53116.1 & $188.9-183.9$ & 316.9 & 318.9 & 136.0 & 4.13" Btoel & $08-18-76$ \\
\hline 1D & 79688.3 & 63114.5 & 222.2-217.2 & 317.2 & 319.1 & 101.9 & 4.13" Bteel & $09-21-76$ \\
\hline $2 A$ & 79243.6 & 65423.8 & 67.1- 63.1 & 288.1 & 290.1 & 237.0 & 4.13" Stoel & $04-01-77$ \\
\hline 2B & 79251.4 & 65424.0 & 83.8. 78.8 & 287.8 & 290.1 & 211.3 & 4.13" Steol & $04-07-77$ \\
\hline $2 C$ & 79259.7 & 65423.7 & 194.6-129.6 & 287.6 & 289.3 & 159.8 & 4.13" Bteel & $04-14-77$ \\
\hline 2D & 79267.2 & 55423.0 & $164.2-159.2$ & 287.2 & 289.0 & 129.8 & $4.13^{\circ}$ Bteel & $04-18-77$ \\
\hline $2 \mathrm{E}$ & 79275.6 & 65423.7 & 193.9-188.9 & 286.9 & 288.8 & 99.9 & 4.13" Steel & $04-21-77$ \\
\hline 25 & 79283.4 & 56428.0 & 222.3-217.3 & 296.3 & 288.1 & 70.8 & $4.19^{n}$ 8teel & $04-22-77$ \\
\hline $\mathbf{3 A}$ & 78726.6 & 67620.0 & 26.5- 21.6 & 269.5 & 271.4 & 249.9 & $4^{\prime \prime}$ Iron & $10-26-77$ \\
\hline $\mathbf{3 B}$ & 78727.7 & 57629.9 & 66.2. 61.2 & 269.2 & 271.3 & 210.1 & $* *$ & $11-16-77$ \\
\hline $3 \mathrm{C}$ & 78728.0 & 57639.0 & $126.0-121.0$ & 269.0 & 270.8 & 149.8 & 4" Iron & $0809-77$ \\
\hline 3D & 78728.4 & 67647.9 & $170.9-165.9$ & 268.9 & 270.9 & 105.0 & $4^{n}$ Iron & $08-22-77$ \\
\hline 3E & 78728.8 & 67665.6 & 190.7-185.7 & 268.7 & 270.7 & 86.0 & $4^{n}$ Iron & $08-30-77$ \\
\hline 37 & 78729.1 & 57663.2 & 210.1-205.1 & 268.1 & 270.2 & 65.1 & $4^{n}$ Iron & $09-13-77$ \\
\hline $4 A$ & 82242.5 & 63896.6 & (23.0. 28.0) & 299.0 & 240.6 & 184.6 & 4.19" Bteel & $03-07-78$ \\
\hline 4B & 82249.0 & 63901.3 & 81.1. 76.1 & 250.1 & 241.1 & 166.0 & 4.13" Btoel & $04-10-78$ \\
\hline $4 C$ & 82265.4 & 59905.9 & $121.3-116.3$ & 239.3 & 241.3 & 125.0 & $4.13^{n}$ 8toel & $04-14-78$ \\
\hline $4 D$ & 82262.2 & 53910.7 & $161.4-146.4$ & 239.4 & 241.4 & 95.0 & 4.13" Stoel & $04-18-78$ \\
\hline $4 \mathrm{E}$ & 82268.9 & 53916.3 & $181.4-176.4$ & 299.4 & 241.6 & 65.1 & $4.13^{n}$ 8toel & $04-19.78$ \\
\hline $\mathbf{5 A}$ & 87988.1 & 54671.7 & $(10.6-16.6)$ & 204.4 & 208.9 & 176.7 & 4.13n Bteel & $04-24-81$ \\
\hline $6 \mathrm{~B}$ & 87997.7 & 54657.6 & 39.6. 34.6 & 204.6 & 207.1 & 172.6 & $4.13^{n}$ Stoel & $04-90-81$ \\
\hline 6C & 88007.6 & 54646.3 & $75.0-70.0$ & 206.0 & 207.8 & 137.8 & 4.13" 8toel & $04-29-81$ \\
\hline 6D & 88011.9 & 54635.7 & $141.4-136.4$ & 206.4 & 208.0 & 71.6 & 4.13" Steel & $04-28-81$ \\
\hline
\end{tabular}

Abandoned

01-26-89

03-15-89

02-03-89

$02-01.89$

01-31.89

01-30-89

Notes: The FCA Beries was known as the FC Beries until 1986 when the name was changed to avoid confusion with the F-Area Hydrology Cluster. See the FCA Berios.

** IC $\mathbf{3 B}$ has 4-in etoel casing innide 6.125-in iron casing. The ccreen is 88 .

\section{FCA WELL SERIES}

Description: F-Area Canyon Building

Bldg. Number: 221-F

Map: Figure A-12

\begin{tabular}{llll} 
& \multicolumn{2}{l}{ 8RS Coordinates } \\
\cline { 3 - 4 } Well D & & North & East \\
FCA 1N & & $\mathbf{7 9 0 3 7 . 3}$ & $\mathbf{5 3 6 7 5 . 9}$ \\
FCA 2C & $\mathbf{7 8 2 9 6 . 0}$ & $\mathbf{5 3 7 1 2 . 2}$ \\
FCA 2D & $\mathbf{7 8 2 9 6 . 8}$ & $\mathbf{6 3 7 1 6 . 2}$ \\
FCA 9B & $\mathbf{7 8 6 9 7 . 9}$ & $\mathbf{6 3 6 7 7 . 1}$ \\
FCA 9C & $\mathbf{7 8 5 9 6 . 7}$ & $\mathbf{5 3 7 1 7 . 6}$ \\
FCA 9D & $\mathbf{7 8 6 0 0 . 6}$ & $\mathbf{5 3 7 3 3 . 1}$
\end{tabular}

(series continued)
Elevations (t MSL)

Brreen Zone Grnd. Casing

298.9-296.8

299.3-296.3

299.0.219.0

297.7.295.2

297.6-295.1

241.9-221.9
$310.3 \quad 812.3$

$310.3 \quad 312.2$

$310.3 \quad 312.2$

$309.9 \quad 309.9$

$310.1 \quad 310.1$

$309.9 \quad 311.9$
Beff. Well

Depth (A) Caring Inotalled Abandonod

$16.5 \quad 4$ " Btreel $10-26-79$

$16.9 \quad 4^{\prime \prime}$ Bteel $\quad 09.20-79$

$93.2 \quad 4^{\prime \prime}$ Bteel $\quad 11.07-79$

$14.7 \quad 4^{\prime \prime}$ Btool $10-31-79$

$15.0 \quad 4^{\prime \prime}$ 8teol $\quad 10-30.79$

$90.0 \quad 4^{\prime \prime}$ Stoel $08-11.80$

\section{Abandoned}


FCA WELL SERIES (cont.)

\begin{tabular}{|c|c|c|c|c|c|c|c|c|c|}
\hline \multirow[b]{2}{*}{ Well ID } & \multicolumn{2}{|c|}{ SRS Coordinates } & \multicolumn{3}{|c|}{ Flevations (At MSL) } & \multirow{2}{*}{$\begin{array}{l}\text { Eff. Well } \\
\text { Depth (ft) }\end{array}$} & \multirow[b]{2}{*}{ Casing } & \multirow[b]{2}{*}{ Inotalled } & \multirow[b]{2}{*}{ Abandoned } \\
\hline & North & Eant & Bcreen Zone & Grnd. & Casing & & & & \\
\hline FCA 10A & 78640.4 & 53571.9 & $241.0-221.0$ & 310.0 & 311.8 & 90.8 & $4^{n}$ Steel & 11.02 .79 & \\
\hline FCA 10B & 78640.9 & 53578.9 & 298.9-294.9 & 909.9 & 311.9 & 17.0 & 4" Bteel & $09.24-79$ & \\
\hline PCA $10 C$ & 78642.5 & 63717.7 & 298.4-295.9 & $\$ 09.9$ & 309.9 & 14.0 & $4^{n}$ Steel & $10-29-79$ & \\
\hline FCA 10D & 78640.0 & 53732.0 & 239.5-219.5 & 309.5 & 311.3 & 91.8 & $4^{*}$ Steel & $10-26-79$ & \\
\hline FCA 16A & 78899.5 & 53568.8 & 236.1-216.1 & 310.4 & 312.2 & 97.1 & $4^{\prime \prime}$ Stoel & $11-06-79$ & \\
\hline FCA 16B & 78898.0 & 63571.0 & $299.3-295.3$ & 310.3 & 312.1 & 16.8 & 4" Steel & 10-19-79 & \\
\hline FCA $16 D$ & 78898.5 & 53719.6 & 241.1-221.i & 310.1 & 310.7 & 89.6 & $4^{n}$ Stool & $03-10-80$ & \\
\hline FCA 16T & 78898.0 & 53579.0 & $292.8-291.3$ & 310.3 & 312.1 & 20.8 & $4^{\prime \prime}$ Stoel & $10-19-79$ & \\
\hline FCA 19D & 78271.9 & 53719.1 & 229.7-209.7 & $\$ 09.7$ & 311.6 & 101.9 & $4^{\circ}$ PVC & $12-19-88$ & \\
\hline
\end{tabular}

Notes: Formerly called FC, this series was changed in 1986 to FCA to avoid confusion with the P-Area Hydrology Cluster Wells.

FCA 28 was drilled to $3 \mathrm{ft}$ on 10-23-79. The drillers hit concrete; coneequently, the well was never inetalled.

FCA 1CC was drillod to $4.6 \mathrm{ft}$ on 10-50-79. The drillere hit concrete; coneequently, FCA $16 \mathrm{C}$ was never inetallod.

\section{FCB WELL SERIES}

Description: F-Area Coal Pile Runoff Containment Basin

Bldg. Number: 289-F

Map: Figures A-8 and A-12

\begin{tabular}{|c|c|c|c|c|c|c|c|c|c|}
\hline \multirow[b]{2}{*}{ Well ID } & \multicolumn{2}{|c|}{ SRS Coondinates } & \multicolumn{3}{|c|}{ Hevations (A MBL) } & Bif. Well & \multirow[b]{2}{*}{ Casing } & \multirow[b]{2}{*}{ Intallot } & \multirow[b]{2}{*}{ Abandoned } \\
\hline & North & Pant & Bcreen 7one & Grnd. & Cening & Depth (f) & & & \\
\hline FCB 1 & 76835.4 & 54871.8 & $235.6-205.6$ & $\$ 05.6$ & 307.6 & 102.0 & $4^{\circ}$ PVC & $10-18-81$ & $07-13-88$ \\
\hline FCB 2 & 76879.7 & 56048.7 & $238.2-206.2$ & 306.2 & 807.3 & 102.1 & $4^{*} \mathrm{PVC}$ & $0908-81$ & \\
\hline FCB 3 & 76427.8 & 54874.4 & $226.3-196.3$ & 300.3 & 302.3 & 107.0 & 4" PVC & $10-16-81$ & \\
\hline FCB 4 & 78780.4 & 54605.9 & $234.6-204.5$ & 304.6 & 308.6 & 102.1 & 4" PVC & $11-04-81$ & \\
\hline FCB 6 & 76492.6 & 54778.0 & 237.1-217.1 & 901.7 & 308.9 & 88.8 & $4^{n}$ PVC & $07-15-88$ & \\
\hline FCB 6 & 76582.1 & B4739.4 & 285.1-216.1 & 308.4 & 310.6 & 96.4 & $4^{\prime \prime P V C}$ & $07-19.88$ & \\
\hline $\mathrm{FCB} 7$ & 76913.9 & 54967.1 & 238.9-218.3 & 318.1 & 315.4 & 97.1 & 4" PVC & $07-0^{4}+8$ & \\
\hline
\end{tabular}

Notes: FCB 1 was firet drillod 9-2-81 and redrilled 10-16-81.

\section{FET WELL SERIES}

Description: F-Area Effluent Treatment Cooling Water Basin Bldg. Number: 241-97F

Map: Figures A-12 and A-13

\begin{tabular}{|c|c|c|c|c|c|c|c|c|c|}
\hline \multirow[b]{2}{*}{ Well ID } & \multicolumn{2}{|c|}{ BR3 Coordinates } & \multicolumn{3}{|c|}{ Elevations (A MSL) } & \multirow{2}{*}{$\begin{array}{l}\text { Eff. Well } \\
\text { Depth (ft) }\end{array}$} & \multirow[b]{2}{*}{ Caning } & \multirow[b]{2}{*}{ Installed } & \multirow[b]{2}{*}{ Abandoned } \\
\hline & North & East & Ecreen Zone & Grnd. & Casing & & & & \\
\hline FLT 1D & 76165.6 & 53299.9 & 226.9.206.9 & 268.0 & 270.0 & 63.1 & 4" PVC & $09-26-88$ & \\
\hline FHT 2D & 76045.8 & 52981.2 & 229.5-209.6 & 267.9 & 270.0 & 60.6 & $4^{*}$ PVC & $08-05-88$ & \\
\hline FET 3D & 75961.0 & 53025.7 & $223.0-209.0$ & 283.2 & 286.2 & 82.2 & 4" PVC & $08-04-88$ & \\
\hline FET 4D & 76959.3 & 53149.0 & 225.1-205.1 & 284.7 & 286.9 & 81.8 & 4" PVC & $08-05-88$ & \\
\hline
\end{tabular}




\section{FMC WELL SERIES}

Description: F-Area Seepage Basins Vertical Radionuclide Distribution Wells Bldg. Number: Not applicable

Map: Figure A-13

\begin{tabular}{|c|c|c|c|c|c|c|c|c|c|}
\hline \multirow[b]{2}{*}{ Well ID } & \multicolumn{2}{|c|}{ SRS Coordinates } & \multicolumn{3}{|c|}{ Elevations (ft MBL) } & \multirow{2}{*}{$\begin{array}{l}\text { Eff. Well } \\
\text { Depth (ft) }\end{array}$} & \multirow[b]{2}{*}{ Casing } & \multirow[b]{2}{*}{ Installed } & \multirow[b]{2}{*}{ Abandoned } \\
\hline & North & East & Bcreen 7one & Grnd. & Casing & & & & \\
\hline IMC & 75561 & 50182 & $164.9-160.9$ & 282.8 & 284.9 & 124.0 & 2" Steel & 11.25 .68 & $03-20-89$ \\
\hline FMC $4 B$ & 75537 & 50201 & $189.3-185.3$ & 281.6 & 283.7 & 98.4 & $2^{n}$ Steel & $11-20-68$ & 03-16-89 \\
\hline
\end{tabular}

Notes: A note on the Well Abandonment Report says that the Program Plan for abandoning MMC 4 C states that the well had an 8-ft ecreen. However, when the well was pulled, there was no screen; tine casing extended the total depth of the weli.

FNB WELL SERIES

Description: Old F-Area Seepage Basin Bldg. Number: 904-49G

Map: Figure A-12

\begin{tabular}{|c|c|c|}
\hline & \multicolumn{2}{|c|}{ SR8 Coordinates } \\
\hline Well IP & North & East \\
\hline NB & 80151.5 & 54271 \\
\hline NB 2 & 80442.3 & 64362 \\
\hline NB 3 & 80663.1 & 64106.8 \\
\hline
\end{tabular}

Dlevatione (ft MSL)

Bcreen 7one Grind. Caning

$207.2-177.2$

210.8-180.8 $286.8 \quad 287.8$

212.1-182.1 $282.1 \quad 284.0$

209.6-179.6 289.6 291.6
Eff. Well

Depth (t)

107.1

107.0

101.9

111.9
Casing

4" PVC

4" PVC

4 " PVC

4" PVC
Inctalled Abandoned

08-09-83

08-11-83

08-16-89

07-24-84

\section{FSB WELL SERIES}

Description: F-Area Seepage Basins

Bldg. Number: 904-41G, -42G, -43G

Map: Figures A-2, A-12, and A-13

\begin{tabular}{|c|c|c|c|c|c|c|c|c|}
\hline \multirow[b]{2}{*}{ Well ID } & \multicolumn{2}{|c|}{ BRs Coordinates } & \multicolumn{3}{|c|}{ Elovations (ft MBL) } & \multirow{2}{*}{$\begin{array}{l}\text { Eff. Well } \\
\text { Depth (t) }\end{array}$} & \multirow[b]{2}{*}{ Caring } & \multirow[b]{2}{*}{ Inotalled } \\
\hline & North & Pact & Screen 7one & Grod. & Cosing & & & \\
\hline P8B 76 & 76141.6 & 61388.8 & 227.0-197.0 & 292.0 & 294.2 & 97.2 & 4" PVC & \\
\hline P8B $76 B$ & 76122.4 & 61394.0 & $\begin{array}{r}47.458 .9 \\
109.7-99.2\end{array}$ & 291.4 & 298.9 & 194.6 & PVC & $10-31-84$ \\
\hline IAB 76C & 76112.4 & 61398.4 & $165.9-164.8$ & 291.3 & 299.6 & 138.8 & PvC & $11-05-84$ \\
\hline PBB 77 & 75129.4 & 50713.1 & 216.4-186.4 & 271.4 & 273.3 & 86.9 & $4^{n}$ PVC & $10-07-81$ \\
\hline F8B 78 & 74764.0 & 50164.7 & 217.7-187.7 & 270.7 & 272.6 & 84.9 & & $10-08-81$ \\
\hline P8B 78A & 74757.7 & 50172.8 & 37.6- 27.0 & 270.5 & 272.6 & 245.6 & PVC & $11-20-84$ \\
\hline F8B 78B & 74765.9 & 50178.8 & 92.8- 82.4 & 270.8 & 272.8 & 190.4 & PVC & $11-28-84$ \\
\hline P8B $78 C$ & 74772.5 & 50170.2 & $161.4-141.6$ & 271.4 & 273.5 & 131.9 & PVC & $11-30-84$ \\
\hline FEB 79 & 73663.1 & 60139.7 & 204.1-174.1 & 216.1 & 217.8 & 43.7 & 4" PVC & $10-09-81$ \\
\hline FAB 79A & 73664.6 & 50149.6 & 34.4- 24.0 & 216.1 & 218.1 & 194.1 & PVC & $11-13-84$ \\
\hline F8B 79B & 73666.1 & 80159.2 & 91.2- 80.7 & 216.2 & 218.2 & 137.5 & PVC & $11-16-84$ \\
\hline $79 \mathrm{C}$ & 73668.0 & 60171.3 & $159.6-149.8$ & 216.6 & 218.4 & 68.6 & PVC & $02-27-84$ \\
\hline
\end{tabular}

(eeries continued) 


\begin{tabular}{|c|c|c|c|c|c|c|c|c|c|}
\hline \multirow[b]{2}{*}{ Well ID } & \multicolumn{2}{|c|}{ gRS Coordinates } & \multicolumn{3}{|c|}{ Elevations (t MSL) } & \multirow{2}{*}{$\begin{array}{l}\text { Eff. Well } \\
\text { Depth (ft) }\end{array}$} & \multirow[b]{2}{*}{ Casing } & \multirow[b]{2}{*}{ Installed } & \multirow[b]{2}{*}{ Abandoned } \\
\hline & North & East & Bcreen Zone & Grnd. & Casing & & & & \\
\hline FBB 87A & 75601.7 & 50116.8 & 43.6- 33.1 & 285.6 & 287.8 & 254.7 & PVC & $10-22-84$ & \\
\hline FSB 87B & 75597.0 & 50104.9 & $100.5 \cdot 90.0$ & 286.5 & 287.5 & 197.5 & PVC & 11.01 .84 & \\
\hline FBB 87C & 76591.9 & 50093.4 & $159.3-148.8$ & 285.3 & 287.5 & 138.7 & PVC & $11-07-84$ & \\
\hline FBB 87D & 75586.3 & 50081.1 & 216.8.187.4 & 286.3 & 287.3 & 99.9 & PVC & $11-16-84$ & \\
\hline FSB 88C & 75619.4 & 51518.0 & $168.4-158.4$ & 280.4 & 283.0 & 124.6 & 4" PVC & $10.07-87$ & \\
\hline FEB 88D & 75621.8 & 51527.0 & 222.1-202.1 & 280.1 & 282.4 & 80.3 & 4" PVC & $10-07-87$ & \\
\hline FSB 89C & 75553.2 & 51346.2 & 166.1.156.1 & 279.1 & 281.3 & 125.2 & 4" PVC & $10-07-87$ & \\
\hline FBB 89D & 76548.3 & 51335.8 & 221.9-201.9 & 278.9 & 281.2 & 79.3 & $4^{n}$ PVC & $10.07-87$ & \\
\hline FSB 9OC & 75382.9 & 51148.6 & 168.1-168.1 & 276.1 & 278.4 & 120.3 & $4^{n}$ PVC & $10-07-87$ & \\
\hline TB 9OD & 75376.9 & 51140.7 & 225.1-205.1 & 276.1 & 278.6 & 73.5 & $4^{n}$ PVC & $10-07-87$ & \\
\hline FSB 91C & 75213.3 & 50953.5 & $159.1-149.1$ & 277.0 & 279.4 & 130.2 & $4^{\prime \prime}$ PVC & $10.07-87$ & \\
\hline F8B 91D & 75207.6 & 50946.6 & $220.9-200.9$ & 276.9 & 279.2 & 78.3 & $4^{n}$ PVC & $10.07-87$ & \\
\hline F8B 92C & 75053.2 & 50564.0 & $157.6-147.6$ & 273.6 & 275.7 & 128.1 & 4" PVC & $10-07-87$ & \\
\hline FBB 92D & 75045.8 & 50557.6 & 221.7-201.7 & 273.7 & 275.9 & 74.2 & $4^{n}$ PVC & $10.07-87$ & \\
\hline FSB 93C & 74897.3 & 50458.3 & $152.0-142.0$ & 274.0 & 276.2 & 134.2 & $4^{n}$ PVC & $10-07.87$ & \\
\hline F8B 93D & 74888.5 & 50452.4 & 217.9-197.9 & 273.9 & 276.1 & 78.2 & $4^{*}$ PVC & $10-07.87$ & \\
\hline FAB 94C & 74869.0 & 50180.0 & $149.8-139.8$ & 278.8 & 281.1 & 141.3 & $4^{N}$ PVC & $10-07-87$ & \\
\hline FBB 94D & 74863.4 & 50188.6 & $221.8-201.8$ & 278.8 & 281.1 & 79.3 & $4^{\prime \prime}$ PVC & $10-07.87$ & $01-23-91$ \\
\hline FSB 94DR & 74869.1 & 50162.9 & 203.4-183.3 & 278.4 & 280.5 & 97.2 & $4^{*}$ PVC & $10.02-90$ & \\
\hline F8B 95C & 74971.7 & 50016.7 & $165.8-146.8$ & 281.8 & 284.0 & 138.2 & $4^{\prime \prime}$ PVC & $10-07-87$ & $01-23-91$ \\
\hline FBB 95CR & 75001.9 & 49987.8 & $161.9-161.9$ & 281.9 & 284.0 & 132.1 & $4^{n}$ PVC & 09.21 .90 & \\
\hline F8B 95D & 74977.6 & 60008.9 & $227.8-207.8$ & 281.8 & 284.3 & 76.5 & $4^{\prime \prime}$ PVC & $10-07.87$ & $01-23-91$ \\
\hline FBB 95DR & 74991.7 & 49996.0 & $207.0-187.0$ & 282.0 & 284.1 & 97.1 & $4^{n}$ PVC & $09-21.90$ & \\
\hline FBB 96A & 74882.2 & 49778.7 & 96.7- 86.7 & 277.7 & 279.8 & 194.1 & $* *$ & $10-07.87$ & $01-23-91$ \\
\hline FGB 96AR & 74914.9 & 49746.6 & 89.0. 79.0 & 279.0 & 281.2 & 202.2 & $4^{n}$ PVC & $09-24-90$ & \\
\hline F8B 97A & 75171.2 & 49965.7 & 95.8- 85.8 & 283.8 & 286.1 & 200.3 & ** & 10.07 .87 & \\
\hline F8B 97C & 75179.6 & 49970.6 & $153.8-143.8$ & 283.8 & 286.1 & 142.3 & $4^{n}$ PVC & $10.07-87$ & \\
\hline FBB 97D & 7518 ค.9 & 49975.5 & 216.9-196.9 & 283.9 & 286.0 & 89.1 & 4" PVC & $10.07-87$ & \\
\hline F8B 98A & 75389.8 & 50121.6 & 94.7. 84.7 & 280.7 & 283.0 & 198.3 & $* *$ & $10-07.87$ & \\
\hline F8B 98C & 76381.2 & 60116.6 & $156.8-146.8$ & 280.8 & 283.1 & 136.3 & $4^{n}$ PVC & $10-07.87$ & \\
\hline FBB 98D & 75371.9 & 50111.6 & 218.8-198.8 & 280.8 & 283.1 & 84.3 & 4" PVC & $10-07.87$ & \\
\hline FBB 99A & 76875.6 & 60314.8 & 102.9-92.9 & 285.3 & 287.6 & 194.7 & $* *$ & $10-07.87$ & \\
\hline TBB 99C & 76683.7 & 50320.6 & 167.2-157.2 & 286.2 & 287.7 & 130.6 & 4" PVC & $10-07-87$ & \\
\hline F8B 99D & 75691.7 & 50326.9 & 218.1-198.1 & 286.1 & 287.6 & 89.5 & 4" PVC & $10.07-87$ & \\
\hline F8B100A & 76534.4 & 50958.4 & 105.8 96.8 & 289.8 & 286.0 & 190.2 & * & $10.07-87$ & \\
\hline F8B101A & 76719.0 & 61191.3 & 102.9- 92.9 & 282.9 & 285.2 & 192.3 & $*$ & $10-07.87$ & \\
\hline F8B102C & 73682.9 & 60834.8 & $165.9-146.9$ & 198.9 & 201.1 & 66.2 & $4^{n}$ PVC & $02-01-88$ & \\
\hline F8B103C & 74210.0 & 49651.3 & $157.1-147.1$ & 240.1 & 242.4 & 96.3 & $4^{n}$ PVC & $02-01.88$ & \\
\hline F8B104C & 73872.6 & 49248.6 & $160.7-150.7$ & 216.7 & 219.1 & 68.4 & $4^{n}$ PVC & $01-20-88$ & \\
\hline F8B104D & 73865.2 & 49256.4 & 210.4-190.4 & 216.9 & 219.2 & 28.8 & 4" PVC & $01.20-88$ & . \\
\hline FSB105C & 75234.2 & 49828.0 & $151.6-141.5$ & 283.5 & 285.8 & 144.3 & 4" PVC & $01-18-88$ & \\
\hline FBB105D & 75244.3 & 49839.3 & 223.7.203.7 & 283.7 & 285.8 & 82.1 & $4^{n}$ PVC & 01.18 .88 & 01.23 .91 \\
\hline F8B106DR & 75258.1 & 49841.0 & 208.6-188.6 & 283.6 & 286.6 & 97.1 & $4^{n}$ PVC & 09.12 .90 & \\
\hline F8B106C & 74190.1 & 50651.3 & 166.0 .156 .0 & 233.0 & 235.1 & 79.1 & $4^{n}$ PVC & 02-05-88 & \\
\hline I8B106D & 74193.0 & 50656.8 & 222.9-202.9 & 232.9 & 234.9 & 32.0 & $4^{n}$ PVC & $06-22-90$ & \\
\hline F8B107C & 75184.0 & 51158.1 & $160.8-160.8$ & 268.8 & 270.9 & 120.1 & $4^{\prime \prime}$ PVC & $01-16-88$ & \\
\hline FSB107D & 75177.2 & 51149.8 & $220.9-200.9$ & 268.7 & 271.0 & 70.1 & $4^{\prime \prime}$ PVC & $01-23-88$ & \\
\hline F8B108D & 76260.7 & 51142.3 & $223.8-203.8$ & 295.8 & 298.0 & 94.2 & $4^{\prime \prime} \mathrm{PVC}$ & $10-07.87$ & \\
\hline F8B109D & 75855.9 & 50488.6 & $225.8-205.8$ & 290.8 & 293.1 & 87.3 & $4^{n}$ PVC & $10-07-87$ & \\
\hline F8B110C & 74190.7 & 50150.6 & $147.2-137.2$ & 232.2 & 234.6 & 97.3 & $4^{\prime \prime}$ PVC & $09-09-87$ & \\
\hline F8B110D & 74193.3 & 50141.6 & 211.1-191.1 & 232.6 & 234.5 & 49.4 & 4" PVC & $01.28-88$ & \\
\hline F8B111C & 75383.3 & 51526.3 & $169.0-159.0$ & 274.0 & 276.3 & 117.3 & $4^{\prime \prime}$ PVC & $02-05-88$ & \\
\hline F8B111D & 75382.9 & 51516.9 & $221.7-201.7$ & 274.4 & 276.6 & 74.9 & $4^{\prime \prime}$ PVC & $02-05-88$ & \\
\hline F8B112A & 74231.4 & 48809.1 & 91.0 .81 .0 & 227.0 & 229.1 & 148.1 & 4" PVC & $06-22-90$ & \\
\hline F8B112C & 74227.5 & 48794.8 & 139.1-129.1 & 227.1 & 229.1 & 100.0 & $4^{n}$ PVC & $06-29-90$ & \\
\hline
\end{tabular}


FSB WELL SERIES (cont.)

\begin{tabular}{|c|c|c|c|c|c|c|c|c|c|}
\hline & 8Rs Coo & dinates & Blevations ( $f$ & MSL) & & Efr. Well & & & \\
\hline Well II & North & East & Screen Zone & Grnd. & Cacing & Depth (ft) & Casing & Installed & Abandoned \\
\hline IBB112D & 74223.7 & 48780.0 & 208.9-188.9 & 227.6 & 229.6 & 40.7 & 4" PVC & $08-28-90$ & \\
\hline F8B113A & 74167.6 & 51068.1 & 91.281 .0 & 221.3 & 223.2 & 142.2 & 4" PVC & 06-08-90 & \\
\hline I8B113C & 74160.7 & 61084.2 & $164.0-164.0$ & 221.0 & 222.9 & 68.9 & 4" PVC & $06-13-90$ & \\
\hline FBB113D & 74164.8 & 51098.4 & 209.6-189.6 & 220.6 & 222.5 & 32.9 & 4" PVC & $08-14-90$ & \\
\hline P8B114A & 76297.4 & 52048.6 & 106.0-96.2 & 250.0 & 252.0 & 186.8 & 4" PVC & $08-16-90$ & \\
\hline F8B114C & 76288.6 & 62039.8 & $168.0-168.0$ & 250.2 & 252.2 & 94.2 & 4" PVC & $08-20-90$ & \\
\hline F8B114D & 76278.6 & 52018.6 & 217.8-197.7 & 260.2 & 252.2 & 54.6 & $4^{\prime \prime}$ PVC & $06-20-90$ & \\
\hline FBB116C & 72516.6 & 49756.0 & 179.8 .169 .8 & 206.8 & 207.8 & 44.0 & 4" PVC & $07.24-90$ & \\
\hline FBB116D & 72604.3 & 49728.3 & 192.6-182.6 & 206.6 & 208.6 & 26.0 & 4" PVC & $08-17.90$ & \\
\hline F8B116C & 72725.6 & 50646.9 & $170.6-160.6$ & 200.6 & 202.6 & 42.0 & 4" PVC & $07-24-90$ & \\
\hline F8B116D & 72727.4 & 50629.7 & $196.4-186.4$ & 200.9 & 202.9 & 16.5 & 4" PVC & $02-14-90$ & \\
\hline F8B117D & 74070.4 & 50488.8 & 209.7-189.7 & 228.7 & 230.7 & 41.0 & 4" PVC & $07-08-90$ & \\
\hline FEB118D & 74697.9 & 51276.3 & 211.3-191.3 & 241.3 & 243.3 & 62.0 & 4" PVC & $07-03-90$ & \\
\hline F8B119D & 74599.7 & 50600.6 & 213.1-193.1 & 252.1 & 254.1 & 61.0 & 4" PVC & $07-09.90$ & \\
\hline FBB120A & 76638.9 & 49176.7 & $109.0-99.0$ & 278.0 & 280.1 & 181.1 & $4^{\prime \prime}$ PVC & $07.12-90$ & \\
\hline FBB120C & 76549.8 & 49171.1 & $160.7-150.7$ & 277.7 & 279.7 & 129.0 & 4 PVC & $07-17.90$ & \\
\hline IBB120D & 76568.7 & 49163.7 & 216.6-196.6 & 278.6 & 280.6 & 84.0 & 4" PVC & $07-12.90$ & \\
\hline F8B121C & 76166.7 & 48413.1 & 158.4148 .4 & 254.4 & 266.6 & 108.1 & 4 PVC & $07-12.90$ & \\
\hline F8B122C & 73881.8 & 48195.0 & $170.0-160.0$ & 216.0 & 218.0 & $\mathbf{5 8 . 0}$ & $4^{\prime \prime}$ PVC & $06-29-90$ & \\
\hline FBB122D & 73886.5 & 48201.7 & 206.6-186.6 & 216.6 & 217.6 & 91.0 & 4 PVC & $07-24-90$ & \\
\hline F8B123C & 74686.7 & 61760.6 & 166.3.165.3 & 236.3 & 238.1 & 82.8 & 4" PVC & $02-22-90$ & \\
\hline F8B123D & 74682.7 & 61734.8 & 214.1194 .1 & 286.1 & 238.1 & 44.0 & $4^{*}$ PVC & $08-22-90$ & \\
\hline
\end{tabular}

Notas: According to installation and activity roports for FBB 115C, the original well hole wal grouted and abandoned 7.20-90. A well by the same name was inctalled 10 ft from the original bole 7-24-90.

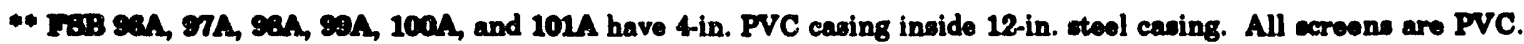

\section{FSB 1TA WELL}

Description: F-Area Seepage Basins Production Well

Bldg. Number: Not applicable

Map: Figure A-12

\begin{tabular}{|c|c|c|c|c|c|c|c|c|}
\hline & g128 Coordinates & Plevationg (f & M(BI) & & Pefr. Well & & & \\
\hline Well m & North Pant & Emeen Zone & Grnd. & Caning & Depth (f) & Caning & Intalled & Abandoned \\
\hline 1TA & 76649.1 & ( & 275.4 & 277.3 & 512.7 & ** & $12-13-88$ & \\
\hline
\end{tabular}

Notea: The Bereen Zone Blevation in parenthenis is in foet below MBL.

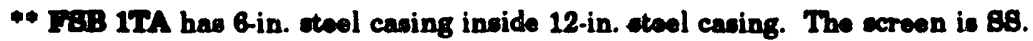


FSS WELL SERIES

Description: F-Area Sludge Land Application Site Bldg. Number: Not applicable

Map: Figures A-12 and A-13

\begin{tabular}{|c|c|c|c|c|c|}
\hline \multirow[b]{2}{*}{ Well ID } & \multicolumn{2}{|c|}{ SRS Coordinates } & \multicolumn{3}{|c|}{ Elevations (ft MSL) } \\
\hline & North & East & Screen Zone & Grnd. & Casing \\
\hline & 75257.6 & 53897.6 & 229.9-209.9 & 269.9 & 266.0 \\
\hline 2D & 75103.5 & 53918.9 & 224.4-204.4 & 259.4 & 261.6 \\
\hline 18 & 74960.5 & 63548.0 & $225.8-205.8$ & 255.8 & 258.2 \\
\hline 00 & 75637.8 & 6.2876.1 & 222.6-202.6 & 289.8 & 291.8 \\
\hline
\end{tabular}

\section{FTF WELL SERIES}

Description: F-Area Tank Farm

Bldg. Number: 241-F, -6F, -11F, -14F, -54F

Map: Figures A-12 and A-13

\begin{tabular}{|c|c|c|c|c|c|c|c|c|c|}
\hline & SRS Coo & linates & Flevations of & MSI) & & Bff. Well & & & \\
\hline Well ID & North & Fast & Screen 2one & Grnd. & Casing & Depth (ft) & Caring & Ingtalled & Abandoned \\
\hline FIT 1 & 77413.3 & 53179.8 & 241.2-221.2 & 281.2 & 283.1 & 61.9 & $4^{n}$ PVC & $08-02-72$ & \\
\hline FTP 2 & 77396.0 & 53276.1 & $299.4-219.4$ & 279.4 & 281.3 & 61.9 & $4^{\circ}$ PVC & $06-16-72$ & \\
\hline ITI 3 & 77235.3 & 53244.8 & 221.2-218.2 & 278.2 & 280.0 & 61.8 & $3^{\omega}$ Stoel & $09-16-72$ & \\
\hline PTP 4 & 77132.9 & 53268.2 & 236.6-216.6 & 276.6 & 278.5 & 61.9 & $4^{\circ}$ FVC & $0808-72$ & \\
\hline PTF 6 & 77035.6 & 53168.3 & 2s5.3-215.3 & 275.3 & 277.3 & 62.0 & $4^{\circ}$ PVC & $08-10-72$ & \\
\hline FTF 6 & 77161.4 & 53062.0 & 2s6.9-216.9 & 276.9 & 278.0 & 61.1 & PVC & $06-09-72$ & \\
\hline FTF 7 & 77235.9 & 53089.7 & 226.1-222.1 & 278.1 & 280.0 & 57.9 & $3^{\infty}$ Stool & $09-08-73$ & \\
\hline FTF 8 & 77936.2 & 53059.9 & 239.6-219.6 & 279.6 & 281.6 & 61.9 & 4" PVC & $06-13-72$ & \\
\hline FTF 9 & 77482.8 & 52769.5 & $236.4-216.4$ & 270.4 & 271.9 & 66.6 & $4^{\prime \prime}$ PVC & $08-18-73$ & \\
\hline FTI 10 & 77396.0 & 52905.0 & 235.1-216.1 & 269.1 & 271.1 & 68.0 & $4^{n}$ PVC & $07-03-72$ & \\
\hline FTP 11 & 77180.7 & 52748.8 & 235.8-216.8 & 269.8 & 271.2 & 65.4 & $4^{n}$ PVC & $07-05-72$ & \\
\hline FII 12 & 77321.4 & 52648.6 & $235.0-215.0$ & 269.0 & 271.7 & 56.7 & $4^{n}$ Eteel & $11-13-73$ & \\
\hline FTF 13 & 76697.8 & 63098.4 & 2s6.1-216.1 & 284.1 & 285.8 & 69.7 & $4^{\prime \prime}$ Steel & $11-17-73$ & \\
\hline FTF 14 & 76188.8 & 52107.7 & 238.6-218.6 & 268.6 & 270.7 & 62.1 & $4^{\circ}$ PVC & $09-10-73$ & \\
\hline FTF 16 & 76732.0 & 63230.0 & 227.6-197.6 & 284.6 & 286.5 & 89.0 & $4^{n}$ PVC & $08-14-84$ & \\
\hline FTP 16 & 76758.6 & 62879.8 & $233.8-203.8$ & 286.8 & 288.6 & 84.8 & 4" PVC & $10-22-84$ & \\
\hline 512 17 & 78872.0 & 62884.0 & $230.6-200.6$ & 287.6 & 289.6 & 89.0 & $4^{\prime \prime}$ PVC & $08-17-84$ & \\
\hline MIF 18 & 76956.8 & 52879.2 & 232.3-202.3 & 286.3 & 288.0 & 86.7 & 4" PVC & $10-16-84$ & \\
\hline MIP 19 & 77139.1 & 52670.4 & 228.3-198.3 & 285.3 & 287.2 & 88.9 & $4^{n}$ PVC & $08-18-84$ & \\
\hline FTY 20 & 77016.0 & 52500.0 & 228.3-198.3 & 285.3 & 287.1 & 88.8 & 4" PVC & $08-21.84$ & \\
\hline FIF 21 & 76866.7 & 62498.6 & 228.7-198.7 & 285.7 & 287.5 & 88.8 & $4^{\prime \prime}$ PVC & $10-25-84$ & \\
\hline FTP 22 & 76751.3 & 52494.7 & 242.6-212.6 & 284.6 & 288.8 & 74.2 & 4" PVC & $08-29-84$ & \\
\hline MrT 23 & 76611.8 & 52660.3 & 231.2-201.2 & 284.2 & 286.0 & 84.8 & $4^{\prime \prime}$ PVC & $0807-84$ & \\
\hline MTP 24 & & & & & & & Steel & $0401-81$ & $01.01 .84^{*}$ \\
\hline FII 24A & 77266.6 & 52780.8 & 232.7.212.7 & 268.4 & 270.3 & 67.6 & $4^{\prime \prime}$ PVC & $08-02-84$ & \\
\hline FIT 26 & & & & & & & Stoel & $0401-81 *$ & $01-01-84^{*}$ \\
\hline MIP 25A & 77308.4 & 52868.7 & 232.8-212.8 & 269.1 & 271.2 & 68.4 & $4^{\prime \prime}$ PVC & $04-30-84$ & \\
\hline FMP 26 & 77250.0 & 52875.4 & 226.3-206.3 & 268.8 & 270.9 & 64.6 & 4" PVC & $05-09-84$ & \\
\hline FII 27 & 77227.2 & 52829.5 & 243.6-213.5 & 268.5 & 270.6 & 57.0 & 4" PVC & $04-20-84$ & \\
\hline
\end{tabular}

Eff. Well

Depth (At)

Installed

Abandoned

$\begin{array}{lll}56.1 & 4^{\prime \prime} \text { PVC } & 10-06-88 \\ 57.2 & 4^{\prime \prime} \text { PVC } & 10-07-88 \\ 52.4 & 4^{\prime \prime} \text { PVC } & 10-10-88 \\ 89.2 & 4^{\prime \prime} \text { PVC } & 10-16-88\end{array}$




\section{GBW WELL SERIES}

Description: Background Well near Hawthorne Fire Tower

Bldg. Number: 627-3G

Map: Figure A-1

\begin{tabular}{|c|c|c|c|c|c|c|c|c|}
\hline & SRg Coordinater & Plevations ( $\mathrm{f}$ & MSL) & & Eff. Well & & & \\
\hline Well ID & North Fast & Bcreen Zone & Grad. & Casing & Depth (ft) & Casing & Ingtalled & Abandoned \\
\hline GBW 1 & 87762.0 & 279.6-249.6 & 332.6 & 333.6 & 84.0 & $4^{\prime \prime}$ PVC & $08-10.82$ & \\
\hline
\end{tabular}

\section{GCD WELL SERIES}

Description: Burial Grounds

Bldg. Number: 643-7E

Map: Not applicable

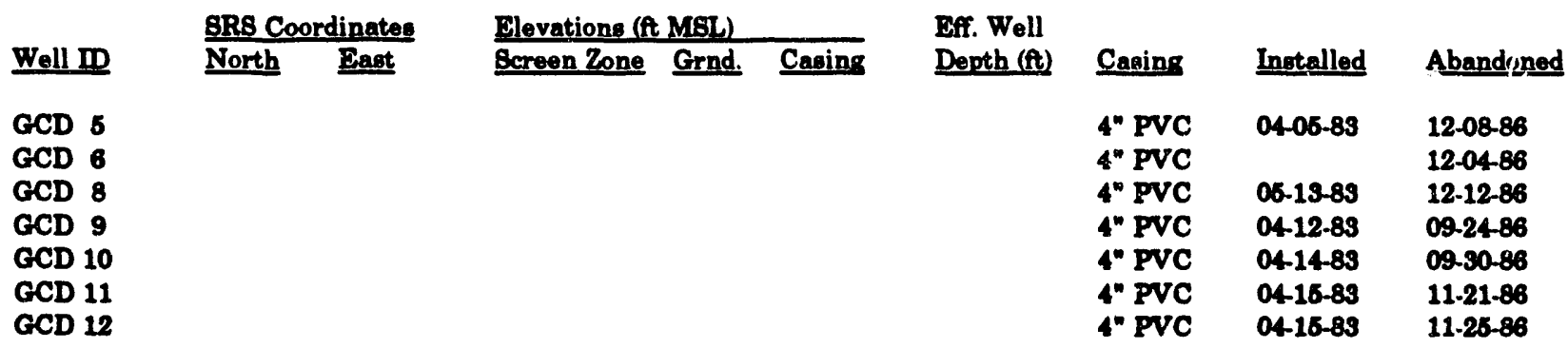

GRID WELL SERIES (see MGA, MGC, MGE, MGG, and MGI WELL SERIES)

\section{H-Area Well}

Description: Unofficial well in $\mathrm{H}$ Area

Bldg. Number: Not applicable

Map: Figure A-15

\begin{tabular}{|c|c|c|c|c|c|}
\hline & BRS Coordinates & Elevations (A MBL) & & & \\
\hline Well ID & North East & Screen Zone Grnd. & Depth (A) & Intalled & Abandoned \\
\hline H 103 & 73350.9 & & & & $07-18-88$ \\
\hline
\end{tabular}

Notes: The identification of this well comes from the abandonment shoets. The $\mathrm{A} 103$ designation is unofficial. 


\section{H WELL SERIES}

Description: H-Area Seepage Basins

Bldg. Number: 904-44G, -45G, -46G, -56G

Map: Figures A-8, A-9, and A-15

\begin{tabular}{|c|c|c|c|c|c|c|c|c|c|}
\hline \multirow[b]{2}{*}{ Well ID } & \multicolumn{2}{|c|}{ BRs Coordinates } & \multicolumn{3}{|c|}{ Flevations (A MSL) } & \multirow{2}{*}{$\begin{array}{l}\text { Eff. Well } \\
\text { Depth (ft) }\end{array}$} & \multirow[b]{2}{*}{ Casing } & \multirow[b]{2}{*}{ Installed } & \multirow[b]{2}{*}{ Abandoned } \\
\hline & North & Enat & Bcreen Zone & Grnd. & Casing & & & & \\
\hline 1 & 72187.4 & 58620.3 & 245.6-235.9 & 262.6 & 263.3 & 27.4 & $3^{n}$ Steel & $03-08-66$ & $03-29-89$ \\
\hline 2 & 72180.5 & 58786.3 & $244.5-234.5$ & 261.6 & 262.9 & 28.4 & 3" Steel & $03-08-56$ & $04-05-89$ \\
\hline 3 & 72098.4 & 58726.6 & $239.0-229.0$ & 259.0 & 259.4 & 30.4 & 3n Steel & $04-24-56$ & $10-01-72$ * \\
\hline 4 & 72071.4 & 68741.3 & 246.2.236.2 & 258.2 & 258.8 & 23.6 & 3n Steel & $03-08-66$ & 04.05 .89 \\
\hline 6 & 72046.9 & 68599.1 & $243.4-233.4$ & 258.4 & 259.1 & 25.7 & 3n Steel & $04-12.56$ & \\
\hline 6 & 72009.1 & 68335.4 & 235.2-225.2 & 259.2 & 260.1 & 34.9 & $3^{n}$ Steel & $04-26-56$ & $04-26-89$ \\
\hline 7 & 71949.2 & 58338.1 & $234.9-224.9$ & 256.9 & 257.8 & 32.9 & 3" Steel & $04-25-56$ & $04-25-89$ \\
\hline 8 & 71616.4 & 58233.9 & 228.4-218.4 & 253.8 & 257.2 & 38.8 & $3^{n}$ Steel & $04-17-56$ & \\
\hline 9 & 71572.6 & 58275.3 & 217.4-207.4 & 246.4 & 248.9 & 41.5 & $3^{n}$ Steel & $04-19-56$ & $04-04-89$ \\
\hline 10 & 71607.2 & 57822.8 & 232.5.222.5 & 254.6 & 257.3 & 34.8 & $3^{n}$ Steel & $05-01-66$ & \\
\hline 11 & 71665.9 & 67779.4 & $222.0-212.0$ & 246.0 & 249.3 & 37.3 & $3^{n}$ Steel & $05-02-66$ & $04-14-8 ?$ \\
\hline 12 & & & & & & & Bteal & $12.31-67$ & \\
\hline 12 & 71829.9 & 58490.3 & & 231.4 & 294.4 & & PVC & $\operatorname{lor} 1-84^{*}$ & \\
\hline 13 & 71327.4 & 58231.7 & & 242.0 & 244.4 & & 2" Jteel & $02-20-62$ & \\
\hline 14 & & & & & & & $2^{n}$ Streel & $02-20-62$ & \\
\hline 14 & 71213.1 & 68071.9 & & 240.3 & 242.3 & & PVC & $3101.85^{*}$ & \\
\hline 16 & 71231.1 & 57812.9 & & 239.6 & 240.1 & & $2^{n} \mathbf{8}$,oel & $02-2062$ & \\
\hline 16 & 71339.8 & 57631.9 & & 240.7 & 241.6 & & 2" Istesl & $02-20-62$ & \\
\hline 17 & 71479.4 & 57682.6 & & 242.4 & 24.3 .3 & & $2^{n}$ Itoul & $02-20-62$ & \\
\hline 18 & 71399.6 & 57337.7 & & & & & 2" Bteel & 02-20-92 & $01-09-86$ \\
\hline $18 A$ & 71339.6 & 57387.7 & 227.5-217.5 & 237.5 & 239.6 & 22.0 & $4^{n}$ PVC & 01.09 .85 & \\
\hline 19 & 71434.2 & 67041.7 & & 243.7 & 246.6 & & Steel & C.2-20-62 & \\
\hline 20 & 71387 & 67180 & & 238.6 & 241.0 & & $3^{n}$ Staol & $04.02-64$ & \\
\hline 21 & 71557 & 56868 & & 246.2 & 247.7 & & $3^{n}$ Steel & $04-02-64$ & \\
\hline 22 & 71652 & 56739 & & 244.0 & 246.6 & & 3" Bteel & $04-02-64$ & \\
\hline 23 & 71770 & 66496 & & 240.3 & 242.8 & & $9^{n}$ Steel & $0402-64$ & \\
\hline 24 & 71943 & 66562 & & 239.5 & 242.0 & & 3" Steel & $04-08-64$ & \\
\hline 26 & 72146 & 56211 & & 240.1 & 242.6 & & 3" Bted & $04-03-64$ & \\
\hline 26 & 71387 & 67490 & & 237.8 & 240.3 & & 3" Stoel & $06-15-64$ & \\
\hline 27 & 71276 & 67246 & & 229.9 & 232.4 & & 3" Steel & $08-16-64$ & \\
\hline 28 & 71323 & 57036 & & 232.8 & 235.3 & & 3" Bteel & $06-15-64$ & \\
\hline 29 & 71438 & 56912 & & 239.8 & 242.3 & & 9" Steel & $08-16-64$ & \\
\hline 30 & 71500 & 58746 & & 238.1 & 240.6 & & $3^{n}$ Steel & $06-16-64$ & \\
\hline 31 & $\because 2642$ & 56589 & & 238.8 & 241.3 & & 3" Btoel & $06-16-64$ & \\
\hline 32 & 71803 & 56340 & & 232.9 & 235.4 & & 3" Steel & $06-16-64$ & \\
\hline 33 & 72024 & 56245 & & 238.6 & 241.1 & & $3^{n}$ Streel & $06-17-64$ & \\
\hline $34 A$ & 71992 & 68696 & & 254.2 & 256.7 & & $2^{n} \mathrm{Al}$ & $07-19-64$ & \\
\hline $34 B$ & 71990 & 58693 & & 254.5 & 257.0 & & 1" Iron & $11-03-64$ & \\
\hline $34 \mathrm{C}$ & 71984 & 58694 & & 256.2 & 257.7 & & 1" Bteel & 07.1464 & \\
\hline 34D & 71986 & 58888 & & 264.6 & 257.0 & & $2^{n}$ Bteel & $11-02-64$ & \\
\hline $\mathbf{3 4 \mathbf { E }}$ & 71984 & 58682 & & 255.1 & 257.6 & & 1* Btoel & $03-24-66$ & \\
\hline 36A & 71901 & 58666 & & 252.0 & 254.5 & & $2^{n} \mathrm{Al}$ & $07-13-64$ & \\
\hline 35B & 71906 & 58566 & & 252.8 & 255.3 & & 1" Iron & $07.16-64$ & \\
\hline $35 \mathrm{C}$ & 71911 & 58652 & & 264.4 & 256.9 & & 1"Iron & $07.16-64$ & \\
\hline 36D & 71918 & 58548 & & 251.7 & 254.2 & & 6" Bteal & $05-18-65$ & \\
\hline 36E & 71926 & 58562 & & 252.3 & 254.8 & & 1" Btoel & 03.21 .66 & \\
\hline $36 \mathrm{~A}$ & 71812 & 58394 & & 248.7 & 251.2 & & $3^{*} \mathrm{Al}$ & $06-2464$ & \\
\hline 36B & 71805 & 58389 & & 249.5 & 252.0 & & 1" Iron & $07-17-64$ & \\
\hline $36 \mathrm{C}$ & 71808 & 68391 & & 249.4 & 251.9 & & 1" Iron & $07-16-64$ & \\
\hline $36 \mathrm{D}$ & 71787 & 68372 & & 249.6 & 252.1 & & 1" Btoel & 0407.66 & \\
\hline
\end{tabular}

(series continued) 
H WELL SERIES (cont.)

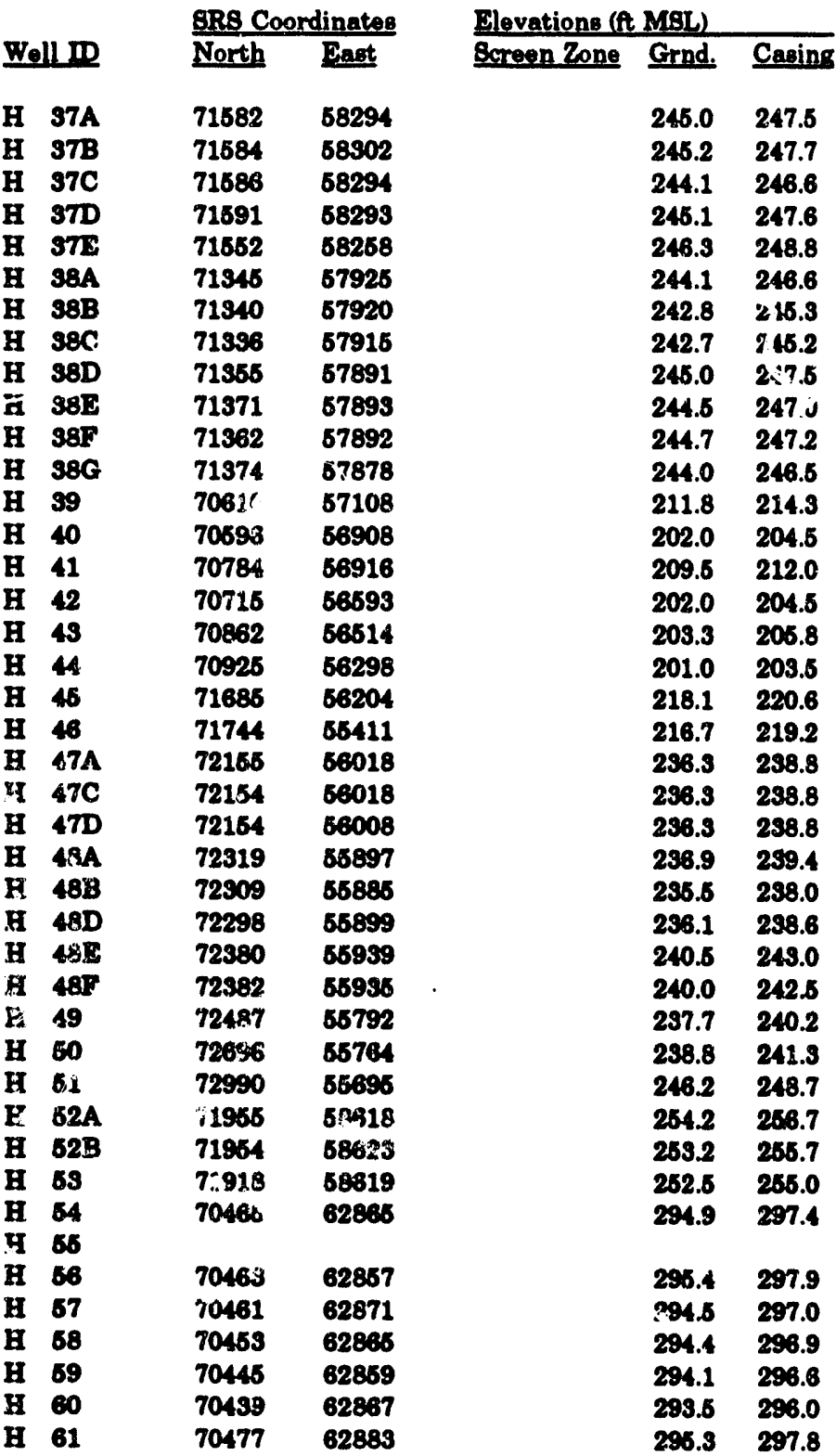

Eff. Well

Depth (ft) Casing Installed Abandonod

$2^{*} \mathrm{Al} \quad 07-13-64$

1"Iron 06-26-64

1" Iron 07-1464

1" Iron 07-23-64

1" Btool 0s-30-66

2" Al 07-22-64

1" Iron 06-30-64

1" Iron 07-22.64

$2^{\text {n Steel 07-12.65 }}$

2" 8toel 07-14-65

1" Stoel 07-08-65

1" Btoel 03-28-66

3" Al 08-0464

$3^{n}$ Al 08-0464

3" Al 08-0464

3" Al 08-06-64

S" Al 08-05-64

3" Al 08-06-64

3" Al 08-04-64

3" Al 08-0464

S" Etool 10-21.64

$2^{n}$ Btoel 11-06-64

1" 8toel 01-26-65

3" Btool 10.21-64

1" Staol 10-0485

1" 8tool 10-19-65

1" Btoel 06-2168

1" steol 06-21-68

3" Btool 10-21-64

$3^{n}$ Stool 10-28-64

$3^{n}$ Bteol 10-26-84

1" Btael 03-07-66

1" Btael 08-17.68

2" Btool 01-0s-68

2" Al 02-2068

4" Al 02-21-68

3" Bteol 02-22-68

1" Bteel 08-0468

1" Steel 02-06-68

1" Etcel 09-0768

2" Bteol 03-1468

Notes: Acconding to the field loge for $\mathbf{B} 18$ and $\mathbf{B}$ 14, now welle were drilled the fourth quarter of 1984 and the firet quartor of 1986, respectively. The data do not indicate if the old wolle wore abandoned, if the w-Il locatione wore changed, or if the caninge wore eimply pulled and repleced.

The Drilling Request for $\mathbf{B} 18 \mathrm{~A}$ indicates that $18 \mathrm{~A}$ was drilled in the same hole as 18. Thirty-nino permanent monitoring wolls (A 13 to $\mathrm{B}$ 51) wore inetalled between 1962 and 1964 (Ponimore and Horton, 1972).

According to well data, $\mathbf{B} 86$ is uncased. 


\section{HAC WELL SERIES}

Description: H-Area Acid/Caustic Basin Bldg. Number: 904-75G

Map: Figure A-14

\begin{tabular}{|c|c|c|c|c|c|c|c|c|}
\hline \multirow[b]{2}{*}{ Well ID } & \multicolumn{2}{|c|}{ BRS Coordinates } & \multicolumn{3}{|c|}{ Elevations (ft MSL) } & \multirow{2}{*}{$\begin{array}{l}\text { Eff. Well } \\
\text { Depth (t) }\end{array}$} & \multirow[b]{2}{*}{ Casing } & \multirow[b]{2}{*}{ Installed } \\
\hline & North & Eagt & Screen Zone & Grnd. & Casing & & & \\
\hline$A C$ & 72171.0 & 61415.2 & 278.8-258.8 & 296.4 & 298.4 & 39.6 & 4" PVC & $08-11-88$ \\
\hline HAC 2 & 72220.2 & 61366.9 & $278.8-258.8$ & 296.2 & 298.1 & 39.3 & 4" PVC & $08-09-88$ \\
\hline HAC 9 & 72183.4 & 51313.6 & $275.0-255.0$ & 295.4 & 298.0 & 43.0 & 4" PVC & $08-18-88$ \\
\hline HAC 4 & 72120.3 & 61372.0 & 274.1-254.1 & 294.7 & 296.9 & 42.8 & 4" PVC & $08-18-88$ \\
\hline
\end{tabular}

\section{HAP WELL SERIES}

Description: H-Area Auxiliary Pump Pit

Bldg. Number: 241-49H

Map: Figure A-14

\begin{tabular}{|c|c|c|c|c|c|c|c|c|c|}
\hline \multirow[b]{2}{*}{ Well ID } & \multicolumn{2}{|c|}{ ERs Coordinates } & \multicolumn{3}{|c|}{ Elevations (ft MBL) } & \multirow{2}{*}{$\begin{array}{l}\text { Bff. Well } \\
\text { Depth (ft) }\end{array}$} & \multirow[b]{2}{*}{ Caging } & \multirow[b]{2}{*}{ Installed } & \multirow[b]{2}{*}{ Abundoned } \\
\hline & North & Bast & Ecreen Zone & Grnd. & Caping & & & & \\
\hline $\begin{array}{ll}\text { HAP } & 1 \\
\text { HAP } 2\end{array}$ & $\begin{array}{l}71209.8 \\
71122.9\end{array}$ & $\begin{array}{l}63598.8 \\
63519.8\end{array}$ & $\begin{array}{l}276.3-256.3 \\
263.8-243.8\end{array}$ & $\begin{array}{l}287.9 \\
287.9\end{array}$ & $\begin{array}{l}289.1 \\
289.9\end{array}$ & $\begin{array}{l}32.8 \\
46.1\end{array}$ & $\begin{array}{l}4^{\prime \prime} \text { PVC } \\
4^{\prime \prime} \text { PVC }\end{array}$ & $\begin{array}{l}11-04-87 \\
11-04-87\end{array}$ & \\
\hline
\end{tabular}

\section{HC WELL SERIES}

Description: H-Area Hydrology Cluster

Bldg. Number: Not applicable

Map: Figures A-8, A-14, A-15, and A-16

\begin{tabular}{|c|c|c|c|c|c|c|c|c|c|}
\hline \multirow{2}{*}{ Well ID } & \multicolumn{2}{|c|}{ SRs Coordinates } & \multicolumn{3}{|c|}{ Slevations (ft MSL) } & \multirow{2}{*}{$\begin{array}{l}\text { Eff. Well } \\
\text { Depth (ft) }\end{array}$} & \multirow[b]{2}{*}{ Caring } & \multirow[b]{2}{*}{ Intalled } & \multirow[b]{2}{*}{ Abandoned } \\
\hline & North & East & Screen Zone & Grad. & Caniog & & & & \\
\hline 1A & 71765 & 61867 & 94.6- 89.6 & 299.5 & 300.8 & 211.3 & 6" Iron & $12-02-64$ & \\
\hline 1B & 71745 & 61877 & $138.5-139.6$ & 299.5 & 800.9 & 167.4 & $6^{n}$ Iron & $12-1464$ & \\
\hline $1 \mathrm{C}$ & 71746 & 61872 & $188.6-183.6$ & 299.6 & 300.7 & 117.2 & $6^{\prime \prime}$ Iron & $12-1864$ & \\
\hline $\mathrm{HC}$ & 71746 & 61867 & 211.5-206.5 & 299.6 & 301.2 & 94.7 & $6^{n}$ Iron & $01-15-65$ & \\
\hline HC & 71746 & 61864 & $256.6-251.6$ & 299.5 & 301.6 & 50.0 & $6^{n}$ Iron & $01-16-65$ & \\
\hline HC & 71746 & 61862 & & 299.5 & 301.4 & & $6^{n}$ Iron & $01-15-66$ & \\
\hline $2 A$ & 71794 & 61866 & 77.2. 72.2 & 298.7 & 300.3 & 228.1 & 6" Iron & $02.09-66$ & \\
\hline HC & 71785 & 61876 & $90.7 \cdot 85.7$ & 298.7 & 300.0 & 214.3 & 6" Iron & $02-24-65$ & \\
\hline $\mathrm{HC}$ & 71784 & 61872 & $140.7-135.7$ & 298.7 & 900.2 & 164.6 & $6^{n}$ Iron & $03-11-65$ & \\
\hline 2D & 71784 & 61866 & 189.2-178.2 & 298.7 & 300.3 & 122.1 & $6^{*}$ Iron & $03-18-65$ & \\
\hline HC & 71784 & 61861 & 210.7-205.7 & 298.7 & 300.5 & 94.8 & $6^{n}$ Iron & $03-24-65$ & \\
\hline HC & 71780 & 61861 & 255.7-250.7 & 298.7 & 300.3 & 49.6 & $6^{n}$ Iron & $03-29-65$ & \\
\hline $\mathrm{HC}$ & & & & & & & Bteel & $04-05-65$ & $04-05-65$ \\
\hline $\mathrm{HC}$ & 71804 & 61862 & $164.7-154.7$ & 298.7 & 300.0 & 145.3 & $6^{\prime \prime}$ Iron & $04-12-65$ & \\
\hline HC & 71742 & 62268 & 70.6- 65.6 & 300.6 & 303.5 & 237.9 & 6" Iron & $03-03-65$ & \\
\hline HC 38 & 71738 & 62253 & 109.1-104.1 & 300.6 & 303.2 & 199.1 & $6^{n}$ Iron & $03-15-65$ & \\
\hline HC SC & 71743 & 62249 & $129.6-124.6$ & 300.6 & 303.4 & 178.8 & $6^{\circ}$ Iron & $03-24-65$ & $03-24-65$ \\
\hline
\end{tabular}

(series continued) 
HC WELL SERIES (cont.)

\begin{tabular}{|c|c|c|c|c|c|c|c|c|c|c|}
\hline \multirow{2}{*}{\multicolumn{2}{|c|}{ Well ID }} & \multicolumn{2}{|c|}{ SRs Coordinatas } & \multicolumn{3}{|c|}{ Plovations (ft MSL) } & \multirow{2}{*}{$\begin{array}{l}\text { Eff. Well } \\
\text { Depth (A) }\end{array}$} & \multirow[b]{2}{*}{ Casing } & \multirow[b]{2}{*}{ Installed } & \multirow[b]{2}{*}{ Abandoned } \\
\hline & & North & Sact & Ecreen Zone & Grnd. & Casing & & & & \\
\hline HC & SD & 71748 & 62251 & & 300.6 & $\mathbf{3 0 3 . 5}$ & & $6^{n}$ Iron & $04-02-65$ & $04-02-65$ \\
\hline HC & $3 \mathbf{E}$ & 71753 & 62251 & 207.1-202.1 & 300.6 & 303.4 & 101.3 & 6" Iron & $04-06-65$ & \\
\hline HC & $3 \mathbf{F}$ & 71758 & 62253 & 245.6-240.6 & 300.6 & 303.5 & 62.9 & 6" Iron & $04-08-65$ & \\
\hline HC & $4 A$ & 71606 & 63409 & $165.0-150.0$ & 293.0 & 294.7 & 144.7 & $6^{n}$ Iron & $04-22.65$ & \\
\hline HC & $4 B$ & 71596 & 63408 & $206.0-200.0$ & 293.0 & 294.2 & 94.2 & $6^{n}$ Iron & 05.05 .65 & \\
\hline HC & 6A & 73265 & 61710 & $161.6-166.5$ & 294.0 & 295.8 & 199.3 & 6" Iron & $05.06-65$ & \\
\hline HC & $\mathbf{6 B}$ & 73266 & 61705 & $203.0-198.0$ & 294.0 & 296.1 & 98.1 & $6^{n}$ Iron & 05.12 .65 & \\
\hline $\mathrm{HC}$ & $6 A$ & 72150 & 62060 & $161.2-166.2$ & 300.2 & 301.7 & 145.5 & $6^{\prime \prime}$ Iron & $04-26-65$ & \\
\hline HC & $6 B$ & 72150 & 62070 & 215.2-210.2 & 300.2 & 301.9 & 91.7 & $6^{\infty}$ Iron & $04-29.65$ & \\
\hline HC & TA & 74352 & 68992 & 133.7-130.7 & 277.0 & 280.0 & 149.3 & $2^{n}$ Stoel & $04-24-87$ & $01-26-88$ \\
\hline HC & TB & 74537 & 66989 & $199.0-194.0$ & 277.0 & 280.2 & 86.2 & $6^{n}$ Steel & $04-26-67$ & $08-04-88$ \\
\hline HC & BA & 77481.8 & 60058.5 & 16.3- 13.3 & 262.3 & 262.7 & 249.4 & 2" Btoel & $05.16-67$ & \\
\hline HC & $8 B$ & 77487.6 & 60058.4 & 137.6-132.5 & 262.3 & 263.9 & 191.4 & 6" Iron & $05-16-67$ & \\
\hline HC & $8 C$ & 77484.4 & 60065.1 & 192.3-187.3 & 262.3 & 264.2 & 76.9 & $6^{n}$ Iron & $12-01-67$ & \\
\hline HC & 9A & 76136 & 64084 & $126.8-123.8$ & 269.3 & 271.9 & 148.1 & $2^{n}$ Steel & $08-01-67$ & $02-17-88$ \\
\hline HC & 9B & 75136 & 64084 & 179.7-174.7 & 269.3 & 271.6 & 96.9 & 6" Stoel & $06-01.67$ & $02-25-88$ \\
\hline HC & $10 A$ & 76806.7 & 61698.4 & $117.0-114.0$ & 228.0 & 229.3 & 116.3 & $2^{n}$ Steel & $08-08-67$ & \\
\hline HC & $10 B$ & 76801.3 & 61600.1 & $169.8-164.8$ & 228.0 & 230.2 & 65.4 & $6^{n}$ Iron & $06-08-67$ & \\
\hline HC & $11 A$ & 74519 & 62147 & & 263.8 & & & Steel & $08-14-67$ & $08-14-67$ \\
\hline HC & 11B & 74499.6 & 62135.3 & $154.8-151.8$ & 269.8 & 265.2 & 113.4 & $2^{n}$ BteeI & $06-16-67$ & \\
\hline $\mathbf{H C}$ & $11 \mathrm{C}$ & 74496.4 & 62131.4 & $196.8-190.8$ & 263.8 & 265.6 & 74.8 & 6" Iron & $06-16-67$ & \\
\hline HC & 12A & 73187 & 69504 & & 287.8 & & & Stoel & $06-2167$ & $06-21-67$ \\
\hline HC & $12 B$ & 79186.9 & 59488.4 & $182.3-177.3$ & 287.3 & 290.1 & 112.8 & $6^{\infty}$ Iron & $06-23-67$ & \\
\hline HC & $13 A$ & 73994 & 69810 & & 291.3 & & & Stoel & $08-30-87$ & $08-90-67$ \\
\hline HC & $13 B$ & 73894 & 63610 & 198.9-198.3 & 291.3 & 292.1 & 98.8 & $6^{\infty}$ Iron & $07-06-67$ & $03-21-88$ \\
\hline HC & $13 C$ & 79894 & 69610 & 211.4-207.1 & 291.3 & 292.6 & 85.6 & 2" Btool & $07-07-67$ & $02-05-88$ \\
\hline $\mathrm{HC}$ & $14 A$ & 67660 & 60858 & 108.6-103.8 & 268.6 & 269.6 & 165.7 & $2^{\infty}$ Eteol & $08-0967$ & \\
\hline HC & $14 B$ & 67563.0 & 60686.9 & & 268.6 & 271.1 & & $6^{n}$ Iron & $08-09-67$ & \\
\hline & 15A & 70949.6 & 61449.7 & & 294.0 & & & Btoel & $08-17-67$ & 08.17 .67 \\
\hline HC & 16B & 70960.9 & 81444.8 & $168.0-163.0$ & 294.0 & 296.7 & 135.7 & 8" Iron & $08-26-87$ & \\
\hline & 16A & 72596.6 & 65461.8 & 123.1-118.1 & 262.6 & 286.5 & 147.4 & $2^{n}$ Steel & $11-29.67$ & $02-16-88$ \\
\hline HC & 16B & 72596 & 65462 & $187.6-183.6$ & 282.6 & 265.4 & 81.8 & $6^{n}$ Etool & $11-29-67$ & $02-29-88$ \\
\hline & $17 \mathrm{~A}$ & & & & 294.0 & & & Steel & $12-1868$ & $12-18-68$ \\
\hline & 18A & & & & 299.0 & & & Steel & $02-01-68$ & $02-01-68$ \\
\hline & $18 B$ & & & & & & & 6" Iron & $08-24-68$ & $09-24-68$ \\
\hline & 36D & 71918 & 68548 & $\begin{array}{ll}92.8 & 87.8\end{array}$ & 263.8 & 254.2 & 166.4 & 6" Btoel & $06-18-65$ & \\
\hline
\end{tabular}

Notes: Table 7 of Clark (n.d.) citas the following problems with wells in the HC Beries. There was no screen for HC IF because the sereen, zone was cemented. HC 20 was cemented and abandoned. HC 2A and $4 \mathrm{~A}$ had partially cemented rereen zones. EC SC was "stopped up." HC SD had a bailor stuck noar the bottom of the caning. BC 11A, 12A, 18A, 15A, 17A, and $18 \mathrm{~A}$ had holes that wore filled with grout. BC $14 B$ was not careened. BC $18 B$ caved in while crews were attempting to and pack. 


\section{HCA WELL SERIES}

Description: H-Area Canyon Building

Bldg. Number: 221-H

Map: Figure A-14

\begin{tabular}{|c|c|c|c|c|c|c|c|c|c|}
\hline \multirow[b]{2}{*}{ Well ID } & \multicolumn{2}{|c|}{ SRS Coordinates } & \multicolumn{3}{|c|}{ Elevations (ft MSL) } & \multirow{2}{*}{$\begin{array}{l}\text { Eff. Weli } \\
\text { Depth (ft) }\end{array}$} & \multirow[b]{2}{*}{ Casing } & \multirow[b]{2}{*}{ Installed } & \multirow[b]{2}{*}{ Abandoned } \\
\hline & North & Eagt & Bcreen Zone & Grnd. & Caning & & & & \\
\hline HCA & 72521.7 & 63109.0 & 273.7-253.7 & 307.7 & 310.0 & 56.3 & $4^{\prime \prime}$ PVC & $12-18-86$ & \\
\hline HCA 2 & 72265.9 & 62943.3 & 273.4-242.0 & 308.4 & 310.8 & 68.8 & 4" PVC & $05-20-86$ & \\
\hline HCA 3 & 72651.7 & 63108.7 & 273.8-253.8 & 307.8 & 310.3 & 56.5 & 4" PVC & $12-11-86$ & \\
\hline HCA 4 & 72523.7 & 62942.9 & 273.3-241.9 & 308.3 & 310.7 & 68.8 & 4" PVC & $05.30-86$ & \\
\hline
\end{tabular}

\section{HCB WELL SERIES}

Description: H-Area Coal Pile Runoff Containment Basin

Bldg. Number: 289-H

Map: Figures A-14 and A-16

\begin{tabular}{|c|c|c|c|c|c|}
\hline \multirow[b]{2}{*}{ Well ID } & \multicolumn{2}{|c|}{ ERS Coordinates } & \multicolumn{3}{|c|}{ Elovations (A MSL) } \\
\hline & North & Egat & Screen Zone & Grnd. & Caning \\
\hline & 71426.8 & 63921.6 & 252.6-222.6 & 277.6 & 279.3 \\
\hline & 71289.7 & 63797.9 & 269.9-239.9 & 279.9 & 281.7 \\
\hline $\mathbf{3}$ & 71098.8 & 63919.9 & 269.6-23s.6 & 273.6 & 276.4 \\
\hline 4 & 71244.2 & 64054.5 & 265.9-235.9 & 275.9 & 277.8 \\
\hline
\end{tabular}

Eff. Woll

Depth (ft)

66.7

41.8

41.8

41.9
Casing

4" PVC

4" PVC

4" PVC

4" PVC
Installed

09-09-81

09-10-81

09-11-81

09-11-81

\section{HCC WELL SERIES}

Description: H-Area Canyon Building

Bldg. Number: 221-H

Map: Figure A-14

\begin{tabular}{llll} 
& \multicolumn{2}{c}{ sRs Coordinates } \\
\cline { 3 - 4 } Well ID & North & East \\
\hline HCC 4 & 72179.7 & 62954.4 \\
HCC 8 & 72222.6 & 62954.1 \\
HCC 6 & 72265.6 & 62954.0 \\
HCC 7 & 72308.4 & 62954.3 \\
HCC 8 & 72361.6 & 62954.1 \\
HCC 9 & 72394.9 & 62954.2 \\
HCC 10 & 72437.6 & 62954.0 \\
HCC 11 & 72480.4 & 62954.4 \\
HCC 12 & 72523.7 & 62959.9 \\
HCC 13 & 72567.1 & 62953.9 \\
HCC 14 & 72609.7 & 62964.0 \\
HCC 15 & 72652.9 & 62954.0 \\
HCC 16 & 72695.6 & 62954.0 \\
HCC 18 & 72736.4 & 63089.6 \\
HCC 19 & 72691.5 & 63089.0
\end{tabular}

Elevations (A MSL) Screen Zone Grnd. Casing

307.2-501.7

$301.2-296.8$

301.8-296.3

303.6-298.1

302.0-296.5

302.1-296.6

301.7-296.2

$302.0-296.6$

301.8-296.3

301.4-295.9

302.5-297.0

301.3-295.8

302.0-296.5

303.7-298.2

302.3-296.8

\begin{tabular}{|c|c|}
\hline 308.7 & 311 \\
\hline 308.8 & 911.0 \\
\hline 908.8 & 911.6 \\
\hline 308.6 & 911.6 \\
\hline 908.0 & 311.0 \\
\hline 308.1 & 311.0 \\
\hline 308.3 & 911.0 \\
\hline 308.4 & 311.1 \\
\hline 308.4 & \$11.1 \\
\hline 308.4 & 311.1 \\
\hline 308.7 & 311.3 \\
\hline 308.3 & 311.0 \\
\hline 308.5 & 311.2 \\
\hline 308.0 & 310.4 \\
\hline 308.0 & 310.7 \\
\hline
\end{tabular}

Eff. Well

Depth (A)

9.4
16.2
15.2
13.6
14.6
14.4
14.8
14.6
14.8
16.2
14.3
15.2
14.7
12.2
13.9

9.4
16.2
16.2
13.6
14.6
14.4
14.8
14.6
14.8
16.2
14.3
15.2
14.7
12.2
13.9

4" PVC

4" PVC

4" PVC PVC 4" PVC 4" PVC 4" PVC 4" PVC 4" PVC 4" PVC 4" PVC 4" PVC 4" PVC 4" PVC PVC
Installed

12-12-86

12-12-86

12-12-86 01-14-87 01-16.87 01.16.87 12-16-86 12-16-86 12-16-86 12-16-86 12-16-86 12-16-86 12-17-86 01-06-87 01-07-87

(series continued) 
HCC WELL SERIES (cont.)

\begin{tabular}{|c|c|c|c|c|c|c|c|c|c|}
\hline \multirow[b]{2}{*}{ Well ID } & \multicolumn{2}{|c|}{ 8RB Coordinates } & \multicolumn{3}{|c|}{ Flevationg (A MSL) } & \multirow{2}{*}{$\begin{array}{l}\text { Eff. Well } \\
\text { Depth (At) }\end{array}$} & \multirow[b]{2}{*}{ Casing } & \multirow[b]{2}{*}{ Inotalled } & \multirow[b]{2}{*}{ Abandoned } \\
\hline & North & Fot & Screen Zone & Grnd. & Casing & & & & \\
\hline HCC 20 & 72651.8 & 63088.9 & 301.1 .296 .6 & 308.1 & 310.6 & 15.0 & 4" PVC & $01-07.87$ & \\
\hline HCC 21 & 72608.6 & 69088.9 & $302.3-296.8$ & 508.1 & 310.6 & 13.8 & 4" PVC & $01-07-87$ & \\
\hline HCC 22 & 72668.2 & 69089.0 & 302.6-297.0 & 307.8 & 310.6 & 13.6 & 4" FVC & $01-09-87$ & \\
\hline HCC 23 & 72521.4 & 68088.9 & $301.6-296.0$ & 308.0 & \$10.4 & 14.4 & $4^{*}$ PVC & $01-09-87$ & \\
\hline ICC 24 & 72481.1 & 63089.1 & $302.2-296.7$ & 308.2 & 311.0 & 14.3 & $4^{*}$ PVC & $01-13-87$ & \\
\hline HCC 25 & 72435.8 & 63088.5 & $302.7-297.2$ & 308.2 & 310.8 & 13.6 & $4^{*}$ PVC & $01-13-87$ & \\
\hline HCC 27 & 72351.0 & 63089.1 & $302.3-296.8$ & 308.0 & 311.1 & 14.3 & $4^{\prime \prime}$ PVC & $01-20-87$ & \\
\hline HCC 28 & 72503.6 & 63087.8 & $309.8-297.8$ & 308.3 & 311.1 & 13.3 & 4" PVC & $01-16-87$ & \\
\hline HCC 31 & 72178.9 & 63089.3 & $301.4-296.9$ & 308.4 & 311.8 & 16.4 & 4" PVC & $01-14-87$ & \\
\hline HCC 32 & 72138.1 & 63089.0 & $302.6-297.1$ & 308.1 & 311.0 & 13.9 & 4" PVC & $01-1487$ & \\
\hline
\end{tabular}

\section{HET WELL SERIES}

Description: H-Area Effluent Treatment Cooling Water Basin Bldg. Number: 241-103H

Map: Figure A-14

\begin{tabular}{|c|c|c|c|c|c|c|c|c|c|}
\hline \multirow[b]{2}{*}{ Well ID } & \multicolumn{2}{|c|}{ BRS Coordinatas } & \multicolumn{3}{|c|}{ Elovatione (ft MSL) } & \multirow{2}{*}{$\begin{array}{l}\text { Def. Woll } \\
\text { Depth (ft) }\end{array}$} & \multirow[b]{2}{*}{ Caring } & \multirow[b]{2}{*}{ Inetalled } & \multirow[b]{2}{*}{ Abandoned } \\
\hline & North & Pant & Ecreen 7one & Gend. & Coning & & & & \\
\hline HET ID & 71948.3 & 60646.0 & $260.3-240.3$ & 279.9 & 282.2 & 41.9 & 4" PVC & 07.29 .88 & \\
\hline HET 2D & 72008.0 & 60094.4 & 263.7-239.7 & 274.8 & 276.9 & 37.2 & 4" PVC & $07-27-88$ & \\
\hline HET SD & 72093.9 & 60110.6 & 269.9-239.9 & 274.6 & 276.7 & 58.8 & 4" PVC & $07.28-88$ & \\
\hline FIST 4D & 72178.1 & 60168.6 & 269.6-239.6 & 274.6 & 276.7 & 37.2 & 4" PVC & $07-21-88$ & \\
\hline
\end{tabular}

\section{HMD WELL SERIES}

Description: Hazardous Waste/Mixed Waste Disposal Facility Bldg. Number:

Map: Figure A-10

\begin{tabular}{|c|c|c|c|c|c|c|c|c|c|}
\hline \multirow[b]{2}{*}{ Well I } & \multicolumn{2}{|c|}{ gRS Coordinates } & \multicolumn{3}{|c|}{ Hovations (t MSL) } & \multirow{2}{*}{$\begin{array}{l}\text { Efr. Woll } \\
\text { Depth (t) }\end{array}$} & \multirow[b]{2}{*}{ Coning } & \multirow[b]{2}{*}{ Installed } & \multirow[b]{2}{*}{ Abandoned } \\
\hline & North & Pant & Ecreen Zone & Gend. & Casing & & & & \\
\hline MD $1 D$ & 78731.7 & 66973.3 & 219.7-199.7 & 262.7 & 284.6 & 64.8 & $4^{*}$ PVC & 02.01 .91 & \\
\hline$M D 2 D$ & 79665.8 & 57269.7 & $210.8-190.8$ & 259.3 & 261.1 & 70.3 & $4^{n}$ PVC & $02.01-91$ & \\
\hline FMD 3D & 79578.7 & 67746.2 & 207.7.187.7 & 267.6 & 269.6 & 71.8 & 4" PVC & 02.01 .91 & \\
\hline FDMD 4D & 79160.4 & 68188.6 & 208.9-188.9 & 248.5 & 260.9 & 62.0 & $4^{n}$ PVC & $02-01.91$ & \\
\hline
\end{tabular}

Notos: EIM 1D, 2D, aD, and $4 \mathrm{D}$ are wator table welle screoned within Aquifor Unit IIB, (McBan formation).

HOR WELL SERIES (see HR3 WELL SERIES) 


\section{HP WELL SERIES}

Description: Upper Tank Farm

Bldg. Number: 241-H

Map: Not applicable

\begin{tabular}{|c|c|c|c|c|c|c|c|c|}
\hline \multirow[b]{2}{*}{ Well II } & SRS Coordinates & \multicolumn{3}{|c|}{ Elevations (ft MSL) } & \multirow{2}{*}{$\begin{array}{l}\text { Eff. Well } \\
\text { Depth (At) }\end{array}$} & \multirow[b]{2}{*}{ Casing } & \multirow[b]{2}{*}{ Installed } & \multirow[b]{2}{*}{ Abandoned } \\
\hline & North East & Screen Zone & Grnd. & Casing & & & & \\
\hline HP 1 & & $273.0-269.0$ & 302.8 & 304.8 & 36.8 & $3^{n} \mathrm{Al}$ & $09-19-60$ & $01.30-85$ \\
\hline HP 3 & & & 302.1 & 304.6 & & $4^{\prime \prime} \mathrm{Al}$ & $09-19.60$ & \\
\hline HP 5 & & 276.2-272.2 & 304.2 & 304.7 & 32.5 & $3^{n}$ Al & 09.19 .60 & $01.29-85$ \\
\hline HP 8 & & 266.7-262.7 & 302.6 & 304.9 & 42.2 & $4^{\prime \prime} \mathrm{Al}$ & $09-21-60$ & $01-30-85$ \\
\hline
\end{tabular}

Notes: According to a 1974 memo, EP 8 was located in a road. The well casing was cut off, and the well was contaminated by surface runoff.

\section{HPM WELL SERIES}

Description: Upper and Lower Tank Farm

Bldg. Number: 241-H

Map: Figures A-14 and A-15

\begin{tabular}{|c|c|c|c|c|c|c|c|c|c|}
\hline \multirow[b]{2}{*}{ Well ID } & \multicolumn{2}{|c|}{ BRS Coordinates } & \multicolumn{3}{|c|}{ Flevations (A MBL) } & \multirow{2}{*}{$\begin{array}{l}\text { Eff. Well } \\
\text { Depth (tt) }\end{array}$} & \multirow[b]{2}{*}{ Caring } & \multirow[b]{2}{*}{ Inotalled } & \multirow[b]{2}{*}{ Abandoned } \\
\hline & North & Eact & Bcreen Zone & Grnd. & Casing & & & & \\
\hline HIPM 1 & 71112.5 & 62232.0 & 270.6-260.6 & 303.0 & 305.8 & 45.2 & $3^{n} \mathrm{Al}$ & $12-02-60$ & $01-29-86$ \\
\hline HPM 2 & 71118.0 & 62192.5 & 271.3-261.3 & 304.2 & 306.6 & 46.3 & $3^{n} \mathrm{Al}$ & 12.07 .60 & $01.23-85$ \\
\hline HPM 3 & 71118.0 & 62143.0 & 269.6-259.5 & 303.3 & 306.3 & 45.8 & $3^{n} \mathrm{Al}$ & $12-08-60$ & 01.26 .86 \\
\hline HPM 4 & 71110.0 & 62043.0 & 266.1-262.1 & 303.3 & 305.3 & 43.2 & $3^{n} \mathrm{Al}$ & $12-15-60$ & $01-26-86$ \\
\hline HPM 5 & 71154.0 & 61961.0 & & 304.8 & 307.3 & & Al & $12-16-60$ & $10-01-77^{*}$ \\
\hline HPM 6 & 71318.0 & 61961.0 & $260.9-260.6$ & $\$ 05.0$ & 306.4 & 46.8 & Al & $12-12-60$ & $01-01-86$ - \\
\hline HPM 7 & 71395.0 & 62043.0 & 274.3-264.s & 304.7 & 307.6 & 49.2 & $\mathbf{A l}$ & $12-08-60$ & $07-18-77$ \\
\hline HPM 8 & 71995.0 & 62160.0 & 267.3-263.3 & 304.3 & 807.3 & 44.0 & $\mathbf{A l}$ & $12-12-60$ & $01-01-86$ - \\
\hline HPM 9 & 71389.0 & 62223.0 & $259.4-259.1$ & 302.6 & 304.7 & 46.6 & $\mathbf{A l}$ & $12-14-60$ & $01-01-85=$ \\
\hline HPM 10 & 71318.0 & 62242.0 & $264.1-260.1$ & 302.8 & 306.0 & 46.9 & $3^{n} \mathrm{Al}$ & $12-06-60$ & $01-18-86$ \\
\hline HPM 11 & 71249.0 & 62242.0 & 271.9-261.9 & 303.1 & 305.4 & 43.6 & $3^{n} \mathrm{Al}$ & $12-0860$ & $01-18-86$ \\
\hline HPM 12 & 71184.0 & 62242.0 & $267.6-263.6$ & 303.4 & 306.2 & 42.6 & $3^{n} \mathrm{Al}$ & $12-06-60$ & $01-22-85$ \\
\hline HPM 13 & 70460 & 62860 & & 295.9 & 298.4 & & $4^{\prime \prime} \mathrm{AI}$ & $03-27-61$ & \\
\hline HPM 14 & 71430 & 62960 & & 297.1 & 299.6 & & $4^{\prime \prime} \mathrm{Al}$ & $09-28-61$ & \\
\hline HPM 15 & 72160 & 62030 & & 298.1 & 300.6 & & $4^{*} \mathrm{Al}$ & $03-27-61$ & \\
\hline HPM 16 & 72670 & 60890 & & 281.7 & 284.2 & & $4^{n} \mathrm{Al}$ & $04-13-61$ & \\
\hline HPM 17 & 71500 & 60950 & & 286.5 & 289.0 & & Al & $04-21-61$ & \\
\hline HPM 18 & 70300 & 60380 & & 289.1 & 291.6 & & $4^{n} \mathrm{Al}$ & $04-21.61$ & \\
\hline HPM 19 & 69270 & 62300 & & 286.9 & 289.4 & & $4^{n} \mathrm{Al}$ & $05-09-61$ & \\
\hline HPM 20 & 70825 & 61835 & & 298.3 & 300.8 & & Al & $04-17.61$ & \\
\hline HPM 21 & 71630 & 62072 & & 277.3 & 279.8 & & $4^{\prime \prime} \mathrm{Al}$ & $07-20-61$ & \\
\hline HIP 22 & 71630 & 62021 & & 279.6 & 282.1 & & $4^{\prime \prime} \mathrm{Al}$ & $07-21-61$ & \\
\hline HPM 23 & 71630 & 61980 & & 279.7 & 282.2 & & $4^{n} \mathrm{Al}$ & $07-21-61$ & \\
\hline HIPM 24 & 71633 & 61956 & & 279.6 & 282.1 & & $4^{n} \mathrm{Al}$ & $07-21-61$ & \\
\hline HPM 25 & 71636 & 62050 & & 279.8 & 282.3 & & $4^{n} \mathrm{Al}$ & $07-21-61$ & \\
\hline HPM 26 & 71638 & 62077 & & 279.7 & 282.2 & & $4^{*} \mathrm{Al}$ & $07-20-61$ & \\
\hline HPM 27 & 71617 & 62080 & & 279.6 & 282.1 & & $4^{n} \mathrm{Al}$ & $07-20-61$ & \\
\hline HPM 28 & 71613 & 62051 & & 279.5 & 282.0 & & $4^{\prime \prime} \mathrm{Al}$ & $07.20-61$ & \\
\hline
\end{tabular}

(series continued) 


\section{HPM WELL SERIES (cont.)}

Notes: BIPA wells 1 through 20 are upper tank farm wells.

BPI 6 was aubject to runoff contamination bessues of being in a contaminated zone according to a 1974 memo.

Ashley, Padezanin, and Zeigler (1984a, 1984b) list monitoring results for HPM 9. Zeigler, Lawrimore, and ORear (1985) indicate that BPY 9 was still in eervice in 1984. Zeigler, Lawrimore, and ORear (1985) aleo give monitoring results for BIP 6 and 8. Although definite abandonment dates are not given, these two welle were probably abandoned in January 1985.

BPA wells 21 through 28 are lower tank farm welle.

\section{HPT WELL SERIES}

Description: Aquifer Characterization Test Wells in $\mathrm{H}$ Area between the Burial Groundis and Road 4

Bldg. Number: Not applicable

Map: Figure A-14

\begin{tabular}{|c|c|c|c|c|c|c|c|c|c|}
\hline & SRs Coo & dinates & Blevations ( $\mathrm{f}$ & MSL) & & Efi. Well & & & \\
\hline Well ID & North & Eant & Screen Zone & Grnd. & Cening & Depth (fi) & Caging & Intalled & Abandoned \\
\hline $\begin{array}{ll}\text { IPS } & 1 A \\
\text { IPT } & 1 B \\
\text { IPT } & \text { 2A }\end{array}$ & $\begin{array}{l}74847.1 \\
74867.4 \\
76061.8\end{array}$ & $\begin{array}{l}60587.0 \\
60590.0 \\
60200.5\end{array}$ & 112.862 .6 & 257.8 & 280.2 & 197.6 & $\begin{array}{l}8^{\infty} \text { PVC } \\
4^{*} \text { PVC } \\
4^{\infty} \text { PVC }\end{array}$ & $\begin{array}{l}10-17-88 \\
10-12-88 \\
10-12-88\end{array}$ & \\
\hline
\end{tabular}

Notes: BPT 1 is a production well for the conotruction of the Mixed Wacte Management Pacility (MWMat clonure cap and is uead as a Congario aquifor characterization test well.

AIPT $1 B$ and $2 A$ are obeervation teat wolls.

HRB WELL SERIES (See the HR8 WELL SERIES)

\section{HR3 WELL SERIES}

Description: Old H-Area Retention Basin

Bldg. Number: 281-3H

Map: Figure A-14

\begin{tabular}{|c|c|c|c|c|c|c|c|c|c|}
\hline & ERS Coo & dinates & Plevations (f & MSL) & & Pfi. Well & & & \\
\hline Well ID & North & Pagt & Ecreen Zone & Grad. & Caring & Depth (ft) & Caning & Intalled & Abandoned \\
\hline & 71402.8 & 60146.5 & $230.0-200.4$ & 269.6 & 271.4 & 71.0 & & $10-16-84$ & \\
\hline HR3 13 & 71649.4 & 60065.6 & $234.8-205.1$ & 274.5 & 276.2 & 71.1 & 4" PVC & $10.18-84$ & \\
\hline
\end{tabular}

Notes: The EDs Beries has been incorrectly referred to as the BOR Sories in the past.

According to a field $\log$ dated 4-15-85, ERS 12 was not drilled. 


\section{HR8 WELL SERIES}

Description: H-Area Retention Basin

Bldg. Number: $281-8 \mathrm{H}$

Mr.: Figures A-8 and A-14

\begin{tabular}{|c|c|c|c|c|c|c|c|c|c|}
\hline \multirow[b]{2}{*}{ Woll M } & \multicolumn{2}{|c|}{ SRs Coordinates } & \multicolumn{3}{|c|}{ Elevations (ft MSL) } & \multirow{2}{*}{$\begin{array}{l}\text { Eff. Well } \\
\text { Depth (At) }\end{array}$} & \multirow[b]{2}{*}{ Casing } & \multirow[b]{2}{*}{ Ingtalled } & \multirow[b]{2}{*}{ Abandoned } \\
\hline & North & East & Bereen Zone & Grnd. & Casing & & & & \\
\hline HR8 11 & 71945.7 & 59559.8 & 237.6-207.9 & 257.4 & 259.2 & 51.3 & 4" PVC & $10-19-84$ & \\
\hline HR8 12 & 71780.1 & 69330.1 & $236.9-206.3$ & 265.7 & 257.5 & 51.2 & 4" PVC & 10.22 .84 & \\
\hline HR8 13 & 71659.6 & 59300.2 & $231.4-201.7$ & 261.2 & 253.1 & 51.4 & $4^{n}$ PVC & $10-22.84$ & \\
\hline FIR8 14 & 71431.4 & 59612.1 & 231.9-202.3 & 251.8 & 253.6 & 51.3 & 4" PVC & $10-23-84$ & \\
\hline
\end{tabular}

Notee: The ERs Berie's has been incorrectly referred to as the ERB Beries in the past.

\section{HSB WELL SERIES}

Description: H-Area Seepage Basins

Bldg. Number: $904-44 G,-45 G,-46 G,-56 G$

Map: Figures A-8, A-10, A-13, and A-14

\begin{tabular}{|c|c|c|}
\hline \multirow[b]{2}{*}{ Well ID } & \multicolumn{2}{|c|}{ BRS Coordinates } \\
\hline & North & Sant \\
\hline & 72425.6 & 68432.C \\
\hline $8 B$ 65A & 72496.2 & 436.0 \\
\hline 8B 65B & 72445.6 & 459.4 \\
\hline IBB 65C & 72439.6 & 58447.1 \\
\hline 8B 66 & 72429.2 & 928.3 \\
\hline $8 B 67$ & 71506.0 & 58424.3 \\
\hline I8B 68 & 71528.0 & 56901.0 \\
\hline H8B 68A & 71526.9 & 56892.1 \\
\hline H8B 68B & 71625.6 & 56882.1 \\
\hline $8 B$ 68C & 71624.1 & 66872.7 \\
\hline BB 69 & 71646.9 & 56475.1 \\
\hline BB 69A & 71549.4 & 66465.1 \\
\hline 8B 70 & 72606.9 & 56758.9 \\
\hline C8B 70C & 72697.3 & 55757.1 \\
\hline [8B 71 & 72875.9 & 65279.2 \\
\hline BB 71C & 72866.6 & 55281.6 \\
\hline SB 83A & 71648.6 & 68606.1 \\
\hline SB 83B & 71639.6 & 58594.9 \\
\hline SB 8SC & 71636.9 & 58614.8 \\
\hline B 83D & 71628.1 & 58601.7 \\
\hline B 84A & 71586.2 & 56359.1 \\
\hline BB 84B & 71603.3 & 66352.4 \\
\hline BB 84C & 71597.1 & 56360.1 \\
\hline 8B 84D & 71583.9 & 56349.9 \\
\hline 8B 85A & 73791.9 & 58943.4 \\
\hline B 85B & 73789.3 & 68963.3 \\
\hline & 73802.3 & 58947.4 \\
\hline & 72520.2 & 65986.9 \\
\hline & 72519.0 & 65976.9 \\
\hline
\end{tabular}

Elevations (ft MSL)

Green Zone Grnd. Caging

$\begin{array}{rrr}242.4-212.4 & 270.4 & 272.0\end{array}$

$\begin{array}{lll}73.2-62.6 & 270.7 & 273.6\end{array}$

$\begin{array}{lll}133.9-123.3 & 270.9 & 273.7\end{array}$

$\begin{array}{lll}218.6-207.8 & 270.6 & 273.6\end{array}$

$\begin{array}{lll}228.1-198.1 & 278.1 & 280.2\end{array}$

$\begin{array}{lll}230.7 .200 .7 & 235.7 & 237.8\end{array}$

$\begin{array}{lll}243.3-213.3 & 248.3 & 260.1\end{array}$

$\begin{array}{lll}\text { 68.0. } 47.5 & 247.4 & 249.4\end{array}$

$\begin{array}{lll}134.5-123.5 & 247.2 & 250.0\end{array}$

$\begin{array}{lll}179.5-168.4 & 246.7 & 260.1\end{array}$

$\begin{array}{lll}229.0-199.0 & 234.0 & 236.0\end{array}$

$\begin{array}{lll}\text { 93.1. } 83.1 & 234.1 & 236.6\end{array}$

$\begin{array}{lll}235.7-205.7 & 240.7 & 242.8\end{array}$

174.9-164.9 $240.9 \quad 243.1$

$\begin{array}{lll}234.8-204.8 & 299.8 & 241.4\end{array}$

$\begin{array}{lll}181.9-171.9 & 239.4 & 241.6\end{array}$

$\begin{array}{lll}\text { 76.0 } 65.2 & 234.9 & 237.3\end{array}$

$\begin{array}{lll}132.1-121.2 & 234.9 & 237.0\end{array}$

$\begin{array}{lll}171.2 .160 .2 & 234.4 & 237.1\end{array}$

$\begin{array}{lll}228.7-198.7 & 234.4 & 237.0\end{array}$

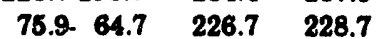

$\begin{array}{lll}132.9-121.8 & 227.0 & 228.9\end{array}$

$\begin{array}{lll}181.8-170.9 & 227.1 & 229.1\end{array}$

$\begin{array}{lll}219.5-199.5 & 225.7 & 228.8\end{array}$

$\begin{array}{lll}71.1-61.1 & 292.1 & 294.4\end{array}$

$\begin{array}{lll}143.2-133.2 & 292.0 & 294.5\end{array}$

$\begin{array}{lll}224.2-214.2 & 292.0 & 294.1\end{array}$

73.9. $63.1 \quad 260.0 \quad 262.4$

$\begin{array}{rrr}124.0-113.8 & 259.6 & 261.9\end{array}$
Bff. Well

Depth (t)

69.6

211.1

160.4

68.8

82.1

37.1

36.8

201.9

126.5

81.7

37.0

163.6

37.1

78.2

36.6

69.7

172.1

115.8

76.9

38.3

164.0

107.1

58.2

29.3

233.3

161.3

79.9

199.3

148.1

\begin{tabular}{ll} 
Caging & Installed \\
\hline 4" PVC & $09-24-81$ \\
4" PVC & $08-01-84$ \\
4" PVC & $08-01-84$ \\
4" PVC & $08-01-84$ \\
4" PVC & $10-14-81$ \\
4" PVC & $09-24-81$ \\
4" PVC & $09-28-81$ \\
4" PVC & $08-01-84$ \\
4" PVC & $07-01-86^{*}$ \\
4" PVC & $07-01-86^{*}$ \\
4" PVC & $09-25-81$ \\
** & $03-07-88$ \\
4" PVC & $09-25-81$ \\
4" PVC & $03-22-88$ \\
4" PVC & $09-29-81$ \\
4" PVC & $03-24-88$ \\
4" PVC & $08-01-84$ \\
4" PVC & $07-01-86^{*}$ \\
4" PVC & $07-01-85^{*}$ \\
4" PVC & $07-01-86^{*}$ \\
4" PVC & $07-01-85^{*}$ \\
4" PVC & $07-01-8 *^{*}$ \\
4" PVC & $07-01-86^{*}$ \\
4" PVC & $07-01-86^{*}$ \\
4" PVC & $07-01-84$ \\
4" PVC & $07-01-84$ \\
4" PVC & $08-01-84$ \\
4" PVC & $08-01-84$ \\
4" PVC & $08-01-84$
\end{tabular}

Abandoned

(cories continued) 
HSB WELL SERIES (cont.)

\begin{tabular}{|c|c|c|c|c|c|c|c|c|c|}
\hline & ERs Coo & dinates & Flevations (f & MSL) & & Eff. Well & & & \\
\hline Well ID & North & Fant & Bcreen Zone & Grnd. & Caging & Depth (t) & Casing & Inotalled & Abandoned \\
\hline HBB $86 C$ & 72529.7 & 66984.6 & $199.4-189.4$ & 260.6 & 262.9 & 73.6 & 4" PVC & $08-01-84$ & \\
\hline HBB 86D & 72522.1 & 65996.5 & 236.6-206.6 & 260.7 & 263.0 & 66.4 & 4" PVC & $08-01-84$ & \\
\hline H8B100C & 72077.2 & 68806.6 & $163.0-163.0$ & 258.0 & 260.2 & 107.2 & 4" PVC & $11-12-87$ & \\
\hline HSB100D & 72073.8 & 68798.9 & 236.9-216.9 & 267.9 & 260.1 & 49.2 & $4^{n}$ PVC & $10-28-87$ & \\
\hline H8B101C & 72001.9 & 68604.4 & 176.3-166.3 & 268.3 & 258.5 & 92.2 & 4" PVC & $11-06-87$ & \\
\hline HSB101D & 71997.6 & 58694.8 & 236.1-216.1 & 268.4 & 258.7 & 42.6 & $4^{\circ}$ PVC & $11-11-87$ & \\
\hline HBB102C & 71960.1 & 68399.7 & 176.7-166.7 & 266.7 & 269.0 & 92.3 & $4^{n}$ PVC & $11-18-87$ & \\
\hline HBB102D & 71962.4 & 68398.4 & 236.3-216.3 & 266.3 & 268.6 & 42.3 & $4^{\prime \prime}$ PVC & $11-19-87$ & \\
\hline H8B10SC & 71698.9 & 58323.6 & 169.2-169.2 & 246.2 & 247.4 & 88.2 & $4^{\prime \prime}$ PVC & $10-30-87$ & \\
\hline ESB103D & 71688.1 & 58316.6 & 233.7-213.7 & 246.4 & 247.6 & 38.9 & $4^{*}$ PVC & $11-02-87$ & \\
\hline H8B104C & 71376.8 & 58082.6 & 173.6-163.6 & 246.5 & 247.9 & 84.4 & $4^{n}$ PVC & $11-09-87$ & \\
\hline HEB104D & 71370.2 & 68076.8 & 250.6-210.6 & 245.6 & 247.8 & 37.2 & $4^{\circ}$ PVC & $10-29-87$ & \\
\hline HBB105C & 71447.3 & 57883.8 & 162.2-162.2 & 247.2 & 249.5 & 97.3 & 4" PVC & $11-0.5-87$ & \\
\hline H8B106D & 71454.8 & 67877.4 & $231.8-211.8$ & 247.2 & 249.6 & 37.7 & $4^{n}$ PVC & $11-06-87$ & \\
\hline H8B108C & 71720.9 & 57661.5 & 168.7-168.7 & 250.7 & 252.9 & 94.2 & 4" PVC & $10-26-87$ & \\
\hline RBB108D & 71727.8 & 67644.8 & $230.7-210.7$ & 250.7 & 262.9 & 42.2 & $4^{n}$ PVC & $10-26-87$ & \\
\hline ESB107C & 71698.6 & 57432.0 & 169.3-169.3 & 259.3 & 261.6 & 102.3 & 4" PVC & $10-26-87$ & \\
\hline FEB107D & 71698.6 & 67412.2 & 235.1-215.1 & 280.0 & 262.3 & 47.2 & 4" PVC & $11-12-87$ & \\
\hline H8B108C & 71688.7 & 67166.5 & 196.0-186.0 & 269.9 & 266.2 & 80.2 & 4" PVC & $10-14-87$ & \\
\hline HSB108D & 71688.0 & 67146.6 & 232.0-212.0 & 264.0 & 268.3 & 54.3 & $4^{n}$ PVC & $10-26-87$ & \\
\hline HBB109C & 71684.8 & 66895.6 & $178.4-168.4$ & 259.4 & 261.6 & 98.2 & $4^{*}$ PVC & 11.11 .87 & \\
\hline I8B109D & 71686.6 & 58886.6 & 233.0-213.0 & 269.0 & 281.2 & 48.2 & $4^{\prime \prime}$ PVC & $11-10-87$ & \\
\hline F8B110C & 71779.3 & 56680.7 & $181.4-171.4$ & 253.4 & 255.7 & 84.3 & 4" PVC & $10-12-87$ & \\
\hline HAB110D & 71785.2 & D6872.1 & $231.4-211.4$ & 268.4 & 255.6 & 4.2 & $4^{*}$ PVC & $10-28-87$ & \\
\hline HBB111C & 71919.4 & 56501.9 & $150.7-140.7$ & 263.7 & 256.0 & 116.9 & $S^{\prime \prime}$ PVC & $11-17-87$ & \\
\hline REB111D & 71928.2 & 68494.6 & 196.7.186.7 & 263.7 & 268.0 & 70.3 & $4^{n}$ FVC & $08-21-87$ & \\
\hline A8B111] & 71982.8 & 664872 & 2s1.7-211.7 & 265.7 & 256.9 & 4.2 & $4^{n}$ PVC & $10-28-87$ & \\
\hline RBB112C & 72166.4 & 68417.4 & $160.6-140.6$ & 252.6 & 264.9 & 114.3 & $4^{n}$ PVC & $11-20-87$ & \\
\hline HEB112D & 72161.6 & 68408.1 & $198.3-188.3$ & 262.8 & 256.1 & 68.8 & $4^{\circ}$ PVC & $10-08-87$ & \\
\hline B8B112E & 72168.6 & cass9.5 & 231.7-211.7 & 262.7 & 265.1 & 48.4 & 4" PVC & $10-27.87$ & \\
\hline A8B113C & 72312.3 & 68160.4 & 161.7-161.7 & 268.7 & 281.0 & 109.3 & $4^{n}$ PVC & $11-24-87$ & \\
\hline HEB118D & 72502.7 & 68184.3 & 256.2-216.2 & 268.7 & 280.9 & 4.7 & $4^{*}$ PVC & $11-20-87$ & \\
\hline HBB114C & 72464.6 & 58107.0 & $198.6-185.6$ & 281.6 & 233.8 & 78.2 & & $10-16-87$ & \\
\hline HBB114D & 72474.2 & 68104.6 & 232.8-212.8 & 261.8 & 284.0 & 61.2 & $4^{\circ} \mathrm{PVC}$ & $10-21-87$ & \\
\hline HBB116C & 72663.2 & 66048.2 & $192.8-182.8$ & 268.8 & 289.3 & 88.6 & $4^{\circ}$ PVC & $10-07-87$ & \\
\hline HAB116D & 72682.3 & 66099.8 & 233.9-213.9 & 268.9 & 269.1 & 66.2 & $4^{*} \mathrm{PVC}$ & $10-20.87$ & \\
\hline HBB116C & 72888.1 & 65980.1 & $190.6-180.6$ & 256.3 & 257.6 & 77.0 & $4^{\circ}$ FVC & $10-20-87$ & \\
\hline FAB116D & 72898.1 & 66888.2 & $294.6-214.6$ & 284.6 & 268.8 & 42.3 & $4^{\circ}$ PVC & $09-02-87$ & \\
\hline H8B117A & 72738.6 & 66170.1 & 94.1. 84.1 & 234.1 & 258.3 & 162.2 & $*$ & $01-25-88$ & \\
\hline HBB117C & 72740.7 & 65162.9 & $174.0-164.0$ & 234.0 & 258.3 & 72.3 & $4^{\circ}$ PVC & $01-27-88$ & \\
\hline H8B117D & 72747.6 & 55165.6 & 219.1-199.1 & 234.1 & 298.3 & 37.2 & 4" PVC & 01.27 .88 & \\
\hline H8B118A & 72696.4 & 65776.6 & 101.0 91.0 & 246.0 & 247.3 & 168.3 & $*$ & $11-25-87$ & \\
\hline HBB119A & 73082.5 & 68100.2 & 108.2- 93.3 & 264.8 & 257.1 & 163.8 & $*$ & $10-05-87$ & \\
\hline HBB120A & 73586.1 & 68431.9 & 101.0 91.0 & 268.0 & 268.2 & 177.2 & $\bullet$ & 11-13-87 & \\
\hline IBB121A & 72024.8 & 67389.6 & 98.3- 88.3 & 272.3 & 274.6 & 188.3 & $*$ & $11-02-87$ & \\
\hline IBB122A & 72196.9 & 67747.4 & $96.4-86.4$ & 269.4 & 271.6 & 188.2 & $*$ & $10-16-87$ & \\
\hline H8B123A & 72189.8 & 58124.8 & 102.3- 92.3 & 282.3 & 284.6 & 172.2 & $*$ & $10-08-87$ & \\
\hline H8B124A & 72199.6 & 58514.6 & 103.0-93.0 & 268.9 & 288.2 & 173.2 & $*$ & $09-16-87$ & \\
\hline HBB125C & 71603.6 & 68592.8 & $155.6-146.6$ & 229.6 & 231.9 & 88.3 & 4" PVC & $03-17-88$ & \\
\hline HAB125D & 71498.2 & 58584.1 & $219.4-199.4$ & 229.4 & 231.7 & 32.3 & 4" PVC & $03-17-88$ & \\
\hline H8B126C & 70627.7 & 57178.2 & $181.3-176.3$ & 210.3 & 212.6 & 36.3 & $4^{\prime \prime}$ PVC & $02-06-88$ & \\
\hline HBB126D & 70633.4 & 57169.6 & $200.6-190.6$ & 210.6 & 212.7 & 22.2 & 4" PVC & $02.06-88$ & \\
\hline H8B127C & 71210.1 & 66792.1 & $158.4-148.4$ & 223.4 & 226.7 & 77.3 & 4" PVC & $03-02-88$ & \\
\hline HSB127D & 71218.9 & 56788.0 & 217.8-197.8 & 223.8 & 228.1 & 28.3 & 4" PVC & $03-02-88$ & \\
\hline H8B129C & 71830.4 & 56110.0 & $157.8-147.8$ & 212.8 & 216.1 & 67.3 & $4^{\prime \prime}$ PVC & $05-18-88$ & \\
\hline
\end{tabular}

(cories continued) 
HSB WELL SERIES (cont.)

\begin{tabular}{|c|c|c|c|c|c|c|c|c|c|}
\hline & SRS Cor & dinates & Dlevations (f & MSL) & & Eff. Well & & & \\
\hline Well ID & North & $\underline{\underline{\text { East }}}$ & Screen Zone & Grnd. & Caring & Depth (ft) & Casing & Installed & Abandoned \\
\hline HSB129D & 71837.1 & 55103.4 & 205.2-185.2 & 212.7 & 214.7 & 29.5 & $4^{\circ} \mathrm{PVC}$ & $03-17-88$ & \\
\hline H8B1soC & 70762.4 & 54643.6 & 169.9-169.9 & 215.9 & 218.3 & 58.4 & 4" PVC & $03-23-88$ & \\
\hline HBB130D & 70757.2 & 54651.7 & 202.1-182.1 & 216.1 & 218.6 & 36.5 & $4^{\prime \prime}$ PVC & $03-23-88$ & \\
\hline H8B191C & 70374.7 & 56894.9 & $168.5-148.6$ & 209.6 & 211.7 & 63.2 & $4^{n}$ PVC & $02-26-88$ & \\
\hline HBB131D & 70365.0 & 56891.1 & 205.7-195.7 & 209.8 & 212.1 & 16.4 & $4^{\prime \prime}$ PVC & $02-26-88$ & \\
\hline HSB132C & 71472.4 & 58787.7 & 178.6-168.6 & 238.3 & 240.5 & 71.9 & 4" PVC & $02-22-88$ & \\
\hline HAB132D & 71469.5 & 58799.3 & 226.5-206.5 & 238.6 & 240.7 & 34.2 & $4^{\prime \prime}$ PVC & $02-22-88$ & \\
\hline HBB133C & 71949.5 & 59110.3 & $183.3-179.3$ & 253.4 & 256.6 & 82.3 & $4^{n}$ PVC & $01-25-88$ & \\
\hline HSB133D & 71943.6 & 69102.3 & 228.5-208.5 & 253.1 & 255.3 & 46.8 & 4" PVC & $01.26-88$ & \\
\hline HBB134C & 71210.3 & 58289.9 & 59.1-149.1 & 236.1 & 238.4 & 89.3 & $4^{\prime \prime}$ PVC & $02-22.88$ & \\
\hline HBB134D & 71217.3 & 58296.5 & 225.8-205.8 & 235.9 & 238.1 & 32.3 & $4^{n}$ PVC & $02.16-88$ & \\
\hline HBB135C & 71390.2 & 56560.8 & 157.3.147.3 & 229.8 & 232.0 & 84.7 & $4^{n}$ PVC & $03-24-88$ & \\
\hline HSB135D & 71396.7 & 56552.8 & 219.9-199.9 & 229.9 & 232.3 & 32.4 & $4^{\prime \prime}$ PVC & $03-11-88$ & \\
\hline HSB136C & 71900.3 & 55949.6 & $170.5 \cdot 160.5$ & 225.6 & 227.9 & 67.4 & 4" PVC & $03-08-88$ & \\
\hline H8B136D & 71906.0 & 56941.7 & 220.2-200.2 & 225.7 & 228.0 & 27.8 & $4^{n}$ PVC & $03-07-88$ & \\
\hline H8B137C & 72269.9 & 55700.2 & $173.8-163.8$ & 233.8 & 236.0 & 72.2 & $4^{n}$ PVC & $03-10-88$ & \\
\hline H8B137D & 72278.9 & 55698.1 & 225.3-205.3 & 234.3 & 236.6 & 31.3 & $4^{n}$ PVC & $03-14-88$ & \\
\hline H8B198D & 73160.2 & 55260.7 & 228.1-208.1 & 250.1 & 252.4 & 44.3 & $4^{\prime \prime}$ PVC & $03-24-88$ & \\
\hline HBB199A & 71127.4 & 57386.4 & 97.6- 87.6 & 231.6 & 233.7 & 146.1 & $* *$ & 02.12 .88 & \\
\hline H8B199C & 71129.8 & 67974.5 & 158.6-148.5 & 231.6 & 233.8 & 86.3 & $4^{\prime \prime}$ PVC & $03-16-88$ & \\
\hline HAB139D & 71133.2 & 67984.4 & 226.7-206.7 & 231.7 & 233.8 & 27.1 & 4" PVC & $02-16-88$ & \\
\hline HSB140A & 70050.3 & 66535.4 & 91.0 81.0 & 234.0 & 235.9 & 154.9 & \&" PVC & 08-03-90 & \\
\hline HSB140C & 70049.2 & 56561.8 & $171.6-161.6$ & 233.6 & 235.6 & 74.0 & 4" PVC & $09-25.90$ & \\
\hline HBB140D & 70036.0 & 56560.6 & 214.1.194.1 & 234.1 & 236.2 & 42.1 & $4^{n}$ PVC & $08-16-90$ & \\
\hline H8B141A & 71213.6 & 59168.7 & $90.6-80.6$ & 252.6 & 254.6 & 174.0 & 4" PVC & $08-16-90$ & \\
\hline $\mathrm{HBB} 141 \mathrm{C}$ & 71196.7 & 69170.2 & $164.7-164.7$ & 252.7 & 264.7 & 100.0 & 4" PVC & $08-02-90$ & \\
\hline H8B141D & 71184.4 & 59170.9 & 237.8-217.8 & 252.8 & 254.8 & 37.0 & $4^{\prime \prime}$ PVC & $08-02-90$ & \\
\hline HBB142C & 73119.0 & 63505.3 & $171.6-161.6$ & 201.6 & 204.0 & 42.4 & $4^{\prime \prime}$ PVC & 11.05 .90 & \\
\hline HSB142D & 73113.0 & 53493.1 & 199.7-189.7 & 201.7 & 204.2 & 14.5 & $4^{n}$ PVC & $11-0.5-90$ & \\
\hline HBB143C & 73738.2 & 62773.2 & 179.1-169.1 & 220.1 & 222.2 & 53.1 & $4^{\prime \prime}$ PVC & $10-09-90$ & \\
\hline H8B143D & 73764.0 & 52774.5 & 216.9-196.9 & 220.9 & 222.9 & 26.0 & 4" PVC & 10.09 .90 & \\
\hline HSB144A & 71892.1 & 56200.5 & 88.6-78.6 & 239.6 & 235.6 & 157.0 & $4^{n}$ PVC & $08-22-90$ & \\
\hline HBB146C & 71098.9 & ธ7769.0 & 174.7-164.7 & 233.7 & 236.7 & 71.0 & $4^{n}$ PVC & 07.31 .90 & \\
\hline HBB145D & 71088.0 & 57753.9 & 194.2-184.2 & 234.2 & 236.2 & 62.0 & $4^{\prime \prime}$ PVC & $08-08-90$ & \\
\hline H8B146C & 70471.6 & 68473.1 & $162.3-152.3$ & 250.9 & 262.3 & 100.0 & $4^{\prime \prime}$ PVC & $08-31-90$ & \\
\hline H8B146D & 70469.7 & 68493.0 & 224.1-204.0 & 261.1 & 253.1 & 49.1 & $4^{\prime \prime}$ PVC & $08-30-90$ & \\
\hline HSB147D & 73827.9 & 55804.4 & 235.2-215.2 & 265.2 & 267.3 & 52.1 & $4^{n}$ PVC & $08-21-90$ & \\
\hline HSB148C & 70151.6 & 55344.2 & $168.9-168.9$ & 248.9 & 260.9 & 92.0 & 4" PVC & $08-07.90$ & \\
\hline H8B148D & 70160.9 & 55355.7 & 218.1-198.1 & 249.1 & 251.1 & $\mathbf{6 3 . 0}$ & 4" PVC & $08-09.90$ & \\
\hline H8B149D & 71338.8 & 57286.3 & 227.0-207.0 & 238.0 & 240.0 & 33.0 & 4" PVC & $07-26-90$ & \\
\hline HSB150D & 71692.6 & 58692.8 & 226.9-206.9 & 236.9 & 239.0 & 32.1 & 4" PVC & $07.26-90$ & \\
\hline H8B161C & 72997.9 & 54014.9 & $180.6-170.6$ & 211.6 & 213.6 & 43.0 & 4" PVC & $08-09-90$ & \\
\hline HSB161D & 72997.8 & 54026.4 & 207.6-197.6 & 211.6 & 213.6 & 16.0 & $4^{n}$ PVC & $08-20-90$ & \\
\hline H8B152C & 72012.0 & 54346.7 & 183.1-173.1 & 212.1 & 214.1 & 41.0 & 4" PVC & 08-19-90 & \\
\hline HSB152D & 72011.7 & 54362.1 & $207.0-197.0$ & 212.0 & 214.1 & 17.1 & 4" PVC & $08-16-90$ & \\
\hline
\end{tabular}

Notes: Geraghty \& Miller (1985) describe the installation of 10 wells in Auguet 1984. The installation date for these welle is designated as 8-01-84.

Christensen and Gordon (1983) refer to wells B8B 65 through 71 as B8W 65 through 71.

HBB 66 was first drilled 9-29-81 and redrilled 10-14-81.

B8B 128 was not drilled.

* E8B 69A, 117A, 118A, 119A, 120A, 121A, 122A, 123A, 124A and 159A have 4-in. PVC casing inside 12-in. oteel casing. All ecreens are PVC. 
HSB 1TB WELL

Description: H-Area Seepage Basins Production Well

Bldg. Number: Not applicable

Map: Figure A.8

\begin{tabular}{|c|c|c|c|c|c|c|c|c|c|}
\hline \multirow[b]{2}{*}{ Well ID } & \multicolumn{2}{|c|}{ 8Rs Coordinates } & \multicolumn{3}{|c|}{ Elevations (ft MSL) } & \multirow{2}{*}{$\begin{array}{l}\text { Eff. Well } \\
\text { Depth (tt) }\end{array}$} & \multirow[b]{2}{*}{ Casing } & \multirow[b]{2}{*}{ Installed } & \multirow[b]{2}{*}{ Abandoned } \\
\hline & North & Padt & Bereen Zone & Grnd. & Casing & & & & \\
\hline H8B 1TB & 72394.0 & 58698.1 & (252.9-295.1) & 267.1 & 269.6 & 864.7 & 6" Stoel & $12-07.88$ & \\
\hline
\end{tabular}

Notes: The Screen Zone Elevation in parenthesie is in feet below MSL.

\section{HSS WELL SERIES}

Description: H-Area Sludge Land Application Site Bldg. Number: Not applicable

Map: Figure A-2

\begin{tabular}{|c|c|c|c|c|c|}
\hline \multirow[b]{2}{*}{ Well ID } & \multicolumn{2}{|c|}{ gRS Coordinates } & \multicolumn{3}{|c|}{ Blovatione (At MSL) } \\
\hline & North & Pant & Exreen 7one & Grad. & Casin: \\
\hline & 67610.3 & 64678.6 & 266.6-236.5 & 308.0 & 310.1 \\
\hline HBS 2D & 67355.9 & 64785.9 & $254.6-234.6$ & 302.3 & 304.4 \\
\hline HBS SD & 68257.5 & 64709.5 & 282.6-262.6 & 308.1 & 309.8 \\
\hline
\end{tabular}

Bif. Well

Dopth (ft)

73.6

69.9

47.2
Caging

4" PVC

4" PVC
Installed

10.29-88

$10-20-88$

$10-27-88$
HSW WELL SERIES (see HSB WELL SERIES)

\section{HTF WELL SERIES}

Description: H.Area Tank Farm

Bldg. Number: 241- $\mathrm{H},-6 \mathrm{H},-8 \mathrm{H},-12 \mathrm{H},-15 \mathrm{H},-83 \mathrm{H}$

Map: Figures A-14 and A-15

\begin{tabular}{|c|c|c|c|c|c|}
\hline \multirow[b]{2}{*}{ Well ID } & \multicolumn{2}{|c|}{ BRs Coordinates } & \multicolumn{3}{|c|}{ Elevation (ft MSI) } \\
\hline & North & Eagt & Sereen rone & Grnd. & Caain \\
\hline HTP 1 & 71745.0 & 62087.0 & 256.9-236.9 & 280.2 & 282.0 \\
\hline HTR 2 & 71610.0 & 62176.0 & $267.0-237.0$ & 280.0 & 281.8 \\
\hline HTP & 71610.0 & 62037.0 & & 278.2 & 280.7 \\
\hline HYT & 71630.0 & 61942.0 & 256.2-235.2 & 280.9 & 282.9 \\
\hline HIT 6 & 71390.0 & 62110.0 & $284.3-284.3$ & 304.3 & 305.8 \\
\hline HTP 6 & 71259.0 & 62228.0 & $289.6-269.6$ & 303.6 & 306.4 \\
\hline $\operatorname{EIF} 7$ & 71130.0 & 62112.0 & $283.6-263.6$ & 303.6 & 305.3 \\
\hline HIY 8 & 71270.0 & 61965.0 & 283.6-263.6 & 503.9 & 305.7 \\
\hline HIT 9 & 71652.0 & 61698.0 & $286.8-246.8$ & 321.7 & 324.0 \\
\hline HIY 10 & 71520.0 & 61838.0 & $266.2-245.2$ & 320.4 & 322.7 \\
\hline HIYP 11 & 71398.0 & 61722.0 & 258.9-238.9 & 320.6 & 322.8 \\
\hline HTY 12 & 71520.0 & 61698.0 & 262.9-242.9 & 320.8 & 322.9 \\
\hline HIT 13 & 71856.0 & 61586.0 & 282.6-262.6 & 322.6 & 324.4 \\
\hline
\end{tabular}

Bff. Woll

Depth (t)

46.1

44.8

47.7

41.6

41.8

41.8

42.1

78.2

77.6

83.9

80.0

61.8

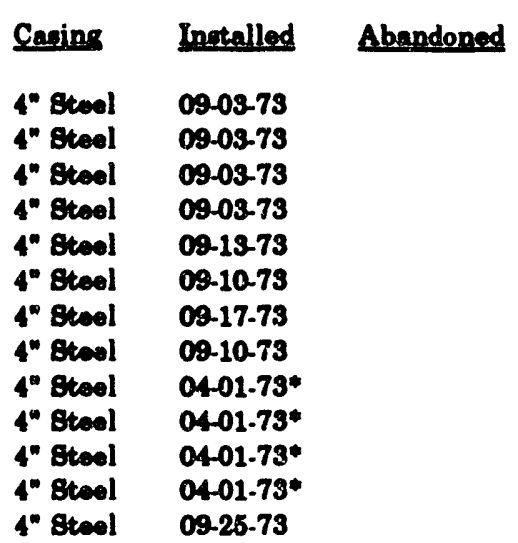

Abandoned

(series continued) 
HTF WELL SERIES (cont.)

\begin{tabular}{|c|c|c|c|c|c|c|c|c|c|}
\hline \multirow[b]{2}{*}{ Well ID } & \multicolumn{2}{|c|}{ SRS Coordinatos } & \multicolumn{3}{|c|}{ Elevations (ft MSL) } & \multirow{2}{*}{$\begin{array}{l}\text { Eff. Well } \\
\text { Depth (ft) }\end{array}$} & \multirow[b]{2}{*}{ Casing } & \multirow[b]{2}{*}{ Installed } & \multirow[b]{2}{*}{ Abandoned } \\
\hline & North & East & Screen Zone & Grnd. & Casing & & & & \\
\hline HITF 14 & 71858.0 & 61462.0 & 281.9-261.9 & 321.9 & 323.9 & 62.0 & $4^{\prime \prime}$ Steel & $09-26-73$ & \\
\hline HTY 16 & 71700.0 & 61353.0 & 280.7-260.7 & 320.7 & 322.5 & 61.8 & $4^{\prime \prime}$ Steel & $09-27.73$ & \\
\hline HTT 16 & 72150.0 & 61950.0 & 268.3-248.9 & 298.4 & 300.3 & 62.0 & Steel & $09.04-73$ & \\
\hline HTF 17 & 72600.0 & 61188.0 & 258.4-238.4 & 288.4 & 25.8 & 61.8 & $4^{\prime \prime}$ PVC & $07-13-73$ & \\
\hline HTF 18 & 71771.8 & 61223.3 & 271.7.251.7 & 321.7 & 323.7 & 72.0 & $4^{n}$ PVC & 05.24 .85 & \\
\hline ETT 19 & 71902.6 & 61079.2 & 265.7-245.7 & 322.7 & 324.8 & 79.1 & $4^{n}$ PVC & $05-30.85$ & \\
\hline HTF 20 & 72073.3 & 61086.4 & 271.9-251.9 & 322.9 & 324.9 & 73.0 & 4" PVC & $08-14-85$ & \\
\hline HIY 21 & 71998.2 & 61261.0 & 262.6-242.6 & 322.6 & 324.7 & 82.1 & PVC & $05-23-85$ & \\
\hline HTF 22 & $7138:$ & 62563.6 & 271.4-251.4 & 331.4 & 333.5 & 82.1 & $4^{n}$ PVC & $03-18-86$ & \\
\hline HTY 23 & 71369.1 & 62670.3 & 276.8-256.8 & 331.8 & 394.0 & 77.2 & 4" PVC & $04-02-85$ & \\
\hline HTF 24 & 71362.6 & 62775.6 & 277.8-257.8 & 931.8 & 333.9 & 76.1 & 4" PVC & $04-04-85$ & \\
\hline FTI 25 & 71224.3 & 62902.0 & 272.5-252.5 & 332.6 & 334.3 & 81.8 & $4^{n}$ PVC & $05-21-85$ & \\
\hline HIT 26 & 71090.7 & 62815.7 & 275.5-255.5 & 333.5 & 395.5 & 80.0 & $4^{n}$ PVC & $06-07-86$ & \\
\hline HIF 27 & 71057.9 & 62660.3 & 279.1-259.1 & 331.1 & 333.1 & 74.0 & 4" PVC & 04-17.85 & \\
\hline HIF 28 & 71080.1 & 62516.7 & 271.9-251.9 & 331.9 & 333.7 & 81.8 & $4^{\prime \prime}$ PVC & $05-10-86$ & \\
\hline HIX 29 & 71229.9 & 62414.9 & 289.9-259.9 & 331.9 & 333.5 & 73.6 & $4^{n}$ PVC & $12.19-84$ & \\
\hline HMF 30 & 70891.6 & 62536.4 & 275.9-255.9 & 327.9 & 329.9 & 74.0 & 4" PVC & $08-07-85$ & $07-01-86^{*}$ \\
\hline HIY 31 & 70747.0 & 62662.5 & 266.7-246.7 & 325.7 & 327.7 & 81.0 & $4^{*}$ PVC & 04-16-85 & \\
\hline HMY 32 & 70880.6 & 62807.9 & 271.1-251.1 & 327.1 & 329.1 & 78.0 & $4^{n}$ PVC & $05-13.85$ & \\
\hline HITF 34 & 71144.1 & 61978.5 & 271.7-251.7 & 303.7 & 305.5 & 63.8 & 4" PVC & $03-13-85$ & \\
\hline
\end{tabular}

\section{HWS WELL SERIES}

Description: Hazardous Waste Storage Facility

Bldg. Number: 709-G, -2G, -4G

Map: Figure A-21

\begin{tabular}{|c|c|c|c|c|c|c|c|c|c|}
\hline \multirow[b]{2}{*}{ Well ID } & \multicolumn{2}{|c|}{ SRS Coordinates } & \multicolumn{3}{|c|}{ Flovations (ft MSL) } & \multirow{2}{*}{$\begin{array}{l}\text { Eff. Well } \\
\text { Depth (t) }\end{array}$} & \multirow[b]{2}{*}{ Casing } & \multirow[b]{2}{*}{ Installed } & \multirow[b]{2}{*}{ Abandoned } \\
\hline & North & East & Sereen Zope & Grnd. & Casing & & & & \\
\hline $\begin{array}{ll}\text { HWS } & 1 \\
\text { HWS } & \text { iA }\end{array}$ & $\begin{array}{l}64885.3 \\
64885.1\end{array}$ & $\begin{array}{l}50223.2 \\
50234.8\end{array}$ & $\begin{array}{l}266.6-246.6 \\
255.2-225.2\end{array}$ & $\begin{array}{l}323.2 \\
323.2\end{array}$ & $\begin{array}{l}325.2 \\
324.6\end{array}$ & $\begin{array}{l}78.6 \\
99.4\end{array}$ & $\begin{array}{l}\text { 4" Btoel }^{n} \text { 4" PVC } \\
4^{n} \text { PVC }\end{array}$ & $\begin{array}{l}03-14-80 \\
07-01-81\end{array}$ & $06-01-82^{*}$ \\
\hline $\begin{array}{ll}\text { HWS } & 2 \\
\text { HWS } & 3\end{array}$ & $\begin{array}{l}64786.3 \\
64664.4\end{array}$ & $\begin{array}{l}50346.4 \\
50194.7\end{array}$ & $\begin{array}{l}246.3-215.3 \\
243.5-213.5\end{array}$ & $\begin{array}{l}320.3 \\
323.5\end{array}$ & $\begin{array}{l}323.2 \\
325.5\end{array}$ & $\begin{array}{l}107.9 \\
112.0\end{array}$ & $\begin{array}{l}4^{\prime \prime} \text { PVC } \\
4^{n} \text { PVC }\end{array}$ & $\begin{array}{l}0.5-31-84 \\
06-01-84\end{array}$ & $05-01-85$ \\
\hline
\end{tabular}

Notes: The HW8 Series was called the CSH Series prior to 1985 in some reports of the raw data. Christensen and Gordon (1983) list these wells as CVB 1 and $1 A$. The designatione for these wells were changed to BWB 1 and $1 A$ in 1985.

According to the field $\log$, HW8 3 was abandoned in May 1985. 


\section{HXB WELL SERIES}

Description: Ford Building Seepage Basin

Bldg. Number: 904-91G

Map: Figure A-21

\begin{tabular}{|c|c|c|c|c|c|c|c|c|c|}
\hline \multirow[b]{2}{*}{ Well ID } & \multicolumn{2}{|c|}{ gR8 Courdinates } & \multicolumn{3}{|c|}{ Blevations (ft MSL) } & \multirow{2}{*}{$\begin{array}{l}\text { Eff. Well } \\
\text { Depth (t) }\end{array}$} & \multirow[b]{2}{*}{ Casing } & \multirow[b]{2}{*}{ Inotalled } & \multirow[b]{2}{*}{ Abandoned } \\
\hline & North & East & Bcreen Zone & Grnd. & Coging & & & & \\
\hline HXB 1 & 60549.7 & 62567.8 & $244.2-214.2$ & 304.2 & 306.2 & 92.0 & $4^{n}$ PVC & $06-24-83$ & \\
\hline HXB 2 & 60866.5 & 62892.8 & 242.1-212.1 & 302.1 & 304.4 & 92.3 & $4^{N}$ PVC & $06-28-83$ & \\
\hline HXB 3 & 60631.2 & 52707.3 & 242.2-212.2 & 302.2 & 304.2 & 92.0 & $4^{n}$ PVC & $06-13-84$ & \\
\hline HXB 4D & 60885.7 & 52617.3 & 254.9-234.9 & 304.4 & 307.0 & 72.1 & 4" PVC & $12-12-90$ & \\
\hline HXB $5 D$ & 60587.7 & 52510.4 & $254.2-234.2$ & 306.2 & 308.8 & 74.6 & A" PVC & $12-07.90$ & \\
\hline
\end{tabular}

\section{IDB WELL SERIES}

Description: Interim Waste Technology Site Characterization Wells, Site B, two miles northeast of $\mathrm{H}$ Area

Bldg. Number: Not applicable

Map: Figure A-23

\begin{tabular}{|c|c|c|c|c|c|c|c|c|c|}
\hline & SRS Coo & linates & Hevations ( $f$ & MBL) & & Bfi. Well & & & \\
\hline Well ID & North & East & Sereen zone & Grnd. & Caging & Depth (ft) & Casing & Installed & Abandoned \\
\hline IDB $1 \mathrm{~A}$ & 72402.7 & 76412.7 & & 293.9 & 296.8 & & & & \\
\hline IDB 18 & 72396.1 & 76407.1 & & 298.8 & 297.3 & & & & \\
\hline IB $1 \mathrm{C}$ & 72388.4 & 76401.0 & & 293.9 & 297.2 & & PVC & 08-26-82 & \\
\hline IDB 2A & 77284.4 & 76391.1 & (22.6. 27.9) & 302.4 & 304.3 & 332.2 & $4^{\prime \prime C B}$ & $01-20-88$ & \\
\hline IDB 2B & 77264.1 & 76592.2 & $121.0-116.0$ & $\$ 08.6$ & 905.7 & 189.7 & $4^{n}$ PVC & 02.01 .88 & \\
\hline $\begin{array}{l}\text { IB } 2 C \\
\end{array}$ & 77244.0 & 75398.6 & $\begin{array}{r}209.3-204.3 \\
76.8 \quad 66.8\end{array}$ & $\begin{array}{l}304.3 \\
304.8\end{array}$ & 308.4 & 102.1 & $\begin{array}{l}4^{n} \text { PVC } \\
\text { 2n PVC }\end{array}$ & 02-09-88 & \\
\hline $\begin{array}{l}\text { DB } \\
\text { IDB }\end{array}$ & $\begin{array}{l}77224.0 \\
77008.6\end{array}$ & $\begin{array}{l}76394.9 \\
76022.1\end{array}$ & $\begin{array}{r}76.868 .8 \\
234.0-229.0\end{array}$ & $\begin{array}{l}304.8 \\
323.0\end{array}$ & 324.9 & $\begin{array}{r}240.8 \\
96.9\end{array}$ & $\begin{array}{l}2^{n} \text { PVC } \\
4^{n} \text { PVC }\end{array}$ & $\begin{array}{l}12-18-90 \\
12-08-87\end{array}$ & \\
\hline IDB 4 & 76134.7 & 74922.9 & $269.6-239.6$ & 314.8 & 316.6 & 77.0 & $4^{n}$ PVC & 12-05-87 & \\
\hline DB 6 & 75670.6 & 75488.8 & 255.1.255.1 & 320.1 & 321.9 & 86.8 & $4^{n}$ PVC & $12-08-87$ & \\
\hline IDB 6 & 72691.3 & 75264.6 & $260.7-240.7$ & 317.0 & 319.0 & 78.3 & \&" PVC & $12-12-87$ & \\
\hline IDB 7 & 73511.9 & 74460.1 & 261.4-241.4 & 311.4 & 318.4 & 72.0 & $4^{n}$ PVC & 12.17 .87 & \\
\hline DB 8 & 72016.7 & 77082.0 & 249.3-229.3 & 291.3 & 298.1 & 69.8 & $4^{n}$ PVC & $12-18.87$ & \\
\hline IDB 9 & 76124.5 & 76398.1 & 206.4-201.4 & 286.4 & 288.3 & 86.9 & $4^{n}$ PVC & $02-06-88$ & \\
\hline DB 10 & 74955.5 & 74406.1 & 220.9-216.9 & 293.9 & 295.8 & 79.9 & $4^{n}$ PVC & $02.09-88$ & \\
\hline
\end{tabular}

Notes: The Bcreen Zone Elevation in parenthesie is in feet below MBL. 


\section{IDP WELL SERIES}

Description: Interim Waste Technology Site Characterization Wells, Site P, adjacent to Site Q, and located south of the TC-1 Area and north of Highway 125

Eldg. Number: Not applicable

Map: Figure A-24

\begin{tabular}{|c|c|c|c|c|c|c|c|c|c|}
\hline \multirow[b]{2}{*}{ WellID } & \multicolumn{2}{|c|}{ gRS Coordinates } & \multicolumn{3}{|c|}{ Flevations (A MBL) } & \multirow{2}{*}{$\begin{array}{l}\text { Eff. Well } \\
\text { Depth (tt) }\end{array}$} & \multirow[b]{2}{*}{ Casing } & \multirow[b]{2}{*}{ Installed } & \multirow[b]{2}{*}{ Abandoner } \\
\hline & North & Past & Scroen Zone & Grnd. & Casing & & & & \\
\hline DPP 1 & 84743.9 & 37883.6 & & 278.6 & 281.0 & & & & \\
\hline $\operatorname{DP} 2$ & 84296.1 & 39527.2 & & 256.5 & 257.9 & & & & \\
\hline IDP $3 A$ & 85104.3 & 37781.1 & (81.3 86.7) & 282.2 & 284.0 & 370.7 & $4^{\prime \prime} \mathrm{CS}$ & $10-29-87$ & \\
\hline $\mathbf{D P} \mathbf{3 B}$ & 85119.6 & 37786.3 & $100.7 \cdot 96.7$ & 282.7 & 284.5 & 188.8 & $4^{*}$ PVC & $9.30-87$ & \\
\hline IDP $\mathbf{3 C}$ & 85139.7 & 37790.1 & 169.1-164.1 & 283.1 & 285.0 & 120.9 & $4^{n}$ PVC & $10.02-87$ & \\
\hline DPP $9 D$ & 85148.4 & $\mathbf{5 7 7 9 4 . 3}$ & $228.8-208.8$ & 283.8 & 285.7 & 76.9 & 4" PVC & $10-06-87$ & \\
\hline DP 4 & 82812.6 & 38615.4 & $199.6-189.5$ & 238.6 & 240.8 & 51.3 & 4" PVC & 11.02 .87 & \\
\hline IDP 5 & 83521.5 & 389.84 .5 & 206.6-186.4 & 251.7 & 263.7 & 67.3 & 4" PVC & $10-30-87$ & \\
\hline IDP 6 & 84113.9 & $3 \& 248.6$ & 209.1-184.6 & 259.6 & 261.5 & 77.0 & 4" PVC & $10-09-87$ & \\
\hline IDP 7 & 84460.1 & 23713.9 & $208.6-188.6$ & 246.6 & 248.6 & 89.9 & $4^{n}$ PVC & $10-07-87$ & \\
\hline IDP 8 & 84740.4 & 39174.3 & 204.5-185.4 & 262.5 & 284.4 & 79.0 & $4^{\prime \prime}$ PVC & $10-08-87$ & \\
\hline IDP 9 & 85951.1 & 37850.4 & 208.0-188.0 & 270.0 & 271.9 & 83.9 & 4" PVC & $10-08-87$ & \\
\hline IDP 10 & 86386.9 & 39280.7 & 208.6.188.6 & 302.3 & 304.3 & 115.7 & $4^{\prime \prime} \mathrm{PVC}$ & $11-23-87$ & \\
\hline
\end{tabular}

Notea: The Bcreen Zone Elevation in parentheeis is in feet below MSL.

\section{IDQ WELL SERIES}

Description: Interim Waste Technolcgy Site Characterization Wells, Site $\mathbf{Q}$, adjacent to Site P, and located south of the TC-1 Area and north of Highway 125

Bldg. Number: Not applicable

Map: Figure A.24

\begin{tabular}{|c|c|c|c|c|c|c|c|c|}
\hline \multirow[b]{2}{*}{ Well m } & \multicolumn{2}{|c|}{ gRS yoordinates } & \multicolumn{3}{|c|}{ Hevations (A MgL) } & Efif. Well & \multirow[b]{2}{*}{ Caning } & \multirow[b]{2}{*}{ Intalled } \\
\hline & North & East & Sereen Tone & Grnd. & Casing & Depth (ft) & & \\
\hline IDQ & 83859.8 & 35866.1 & & & & & & \\
\hline 2 & 81784.5 & 35361.9 & & 261.2 & 263.2 & & & \\
\hline SA & 80553.7 & 35854.0 & (184.3-189.8) & 203.2 & 205.3 & 395.1 & $4^{n} \mathrm{Cs}$ & $11-17.87$ \\
\hline 3B & 80578.4 & 35858.8 & $113.4-108.4$ & 203.7 & 205.6 & 97.2 & 4" PVC & 11.20 .87 \\
\hline IDQ & 80801.7 & 35863.6 & $141.6-136.6$ & 204.6 & 206.5 & 69.9 & $4^{n}$ PVC & $11-20-87$ \\
\hline IDQ 4 & 83126.1 & 36726.2 & 206.6-185.6 & 263.6 & 265.5 & 79.9 & $4^{n}$ PVC & 12.11 .87 \\
\hline IDQ 5 & 82763.6 & 36851.8 & 207.5.187.4 & 265.0 & 267.2 & 79.8 & $4^{n}$ PVC & $11-06-87$ \\
\hline IDQ & 82414.4 & 37299.3 & 202.1-181.9 & 256.1 & 258.1 & 76.2 & $4^{\prime \prime}$ PVC & $11.05-87$ \\
\hline IDQ & 82107.4 & 37856.3 & $194.8-174.6$ & 238.8 & 240.6 & 66.0 & 4" PVC & $11-04-87$ \\
\hline IDQ 8 & 83602.8 & 34688.1 & 200.4180 .4 & 240.7 & 242.6 & 62.2 & 4" PVC & $11.13-87$ \\
\hline IDQ 9 & 82729.6 & 34053.4 & 193.9-173.9 & 294.3 & 236.1 & 62.2 & $4^{n}$ PVC & $11-18-87$ \\
\hline & 82135.8 & 33610.1 & 185.7.165.7 & 234.7 & 236.5 & 70.8 & 4" PVC & $11-19.87$ \\
\hline IDQ 11 & 80360.9 & 34741.0 & $134.8-129.7$ & 206.8 & 208.8 & 79.1 & $4^{\prime \prime}$ PVC & $02.10-88$ \\
\hline IDQ 12 & 81913.7 & 37116.5 & 1/44.9-164.9 & 240.5 & 242.2 & 77.3 & 4" PVC & $02-12$ \\
\hline
\end{tabular}

Notes: The Screen Zone Elevation in parenthesis is in feet below MSL. 


\section{K Well}

Description: Unofficial wells at the Mixed Waste Management Facility (MWMF) Borrow Pit Bldg. Number: Not applicable

Map: Not applicable

\begin{tabular}{|c|c|c|c|c|c|c|c|}
\hline & 8R8 Coordinates & Elevations (ft MSL) & & Eff. Well & & & \\
\hline Well D & North Fast & Bcreen Zone Grnd. & Casing & Depth (ft) & Casing & Installed & Abandoned \\
\hline $\mathbf{K}$ & & & & & $9^{n} \mathrm{Al}$ & & $09-07-88$ \\
\hline
\end{tabular}

Notes: The identification of this well comes from the abandonment sheets. The $\mathbf{K}$ designation is unofficial.

\section{K WELL SERIES}

Description: K-Area Wind Tower Piezometers at B Road

Bldg. Number: Not applicable

Map: Figure A-4

\begin{tabular}{|c|c|c|c|c|c|c|c|c|}
\hline \multirow[b]{2}{*}{ Well ID } & \multicolumn{2}{|c|}{ SR8 Coondinates } & \multicolumn{2}{|l|}{ Glevations (At MSL) } & \multirow{2}{*}{$\begin{array}{l}\text { Eff. Well } \\
\text { Depth (At) }\end{array}$} & \multirow[b]{2}{*}{ Ceaing } & \multirow[b]{2}{*}{ Installed } & \multirow[b]{2}{*}{ Abandoned } \\
\hline & North & Eest & Sereen Zone Grod. & Casing & & & & \\
\hline $\begin{array}{ll}\mathbf{K} & 406 \mathrm{P} \\
\mathbf{K} & 408 \mathrm{P} \\
\mathbf{N} & 91\end{array}$ & $\begin{array}{l}53743 \\
63595\end{array}$ & $\begin{array}{l}36778 \\
37663\end{array}$ & & & & $\begin{array}{l}2^{n} \text { PVC } \\
2^{n} \text { PVC } \\
2^{n} \text { Btoel }\end{array}$ & $\begin{array}{l}06-17-86 \\
06-04-87\end{array}$ & $\begin{array}{l}12-22-89 \\
12-21-89 \\
12-21-89\end{array}$ \\
\hline
\end{tabular}

Notes: Even though the well N 91 does not have the eame well ID (K) as the other welle in the series, this well has been included in the K Well Beries because it was part of the.K.Area Cooling Tower Piezometer Abandonmente Project. All of the wells have the descriptive location.

\section{KAB WELL SERIES}

Description: K-Area Ash Basin

Bldg. Number: 188-K

Map: Figure A-4

\begin{tabular}{|c|c|c|c|c|c|c|c|c|c|}
\hline \multirow[b]{2}{*}{ Well ID } & \multicolumn{2}{|c|}{ BRS Coordinates } & \multicolumn{3}{|c|}{ Elovations (ft MSL) } & \multirow{2}{*}{$\begin{array}{l}\text { Eff. Woll } \\
\text { Depth (A) }\end{array}$} & \multirow[b]{2}{*}{ Caring } & \multirow[b]{2}{*}{ Ingtalled } & \multirow[b]{2}{*}{ Abandoned } \\
\hline & North & East & Screen Zone & Grnd. & Casins & & & & \\
\hline KAB 1 & 53055.6 & 39919.7 & $224.0-194.0$ & 264.0 & 268.0 & 72.0 & 4" PVC & $08-04-83$ & \\
\hline KAB 2 & 52410.8 & 40277.9 & 228.6-198.6 & 258.6 & 260.7 & 62.1 & 4" PVC & $07-18-83$ & \\
\hline KAB 9 & 51807.7 & 39918.4 & $22 \% .0 .193 .0$ & 248.0 & 250.1 & 57.1 & $4^{n}$ PVC & $07.19-83$ & \\
\hline KAB 4 & 52807.1 & 39457.0 & $217.0-187.0$ & 262.0 & 264.4 & 67.4 & 4" PVC & $07.21-83$ & \\
\hline
\end{tabular}


KAC WELL SERIES

Description: K-Area Acid/Caustic Basin

Bldg. Number: 904-80G

Map: Figure A-4

\begin{tabular}{|c|c|c|c|c|c|c|c|c|c|}
\hline \multirow[b]{2}{*}{ Well ID } & \multicolumn{2}{|c|}{ 8Rs Coordinatos } & \multicolumn{3}{|c|}{ Elevations (A MSL) } & \multirow{2}{*}{$\begin{array}{l}\text { Eff. Well } \\
\text { Depth (tt) }\end{array}$} & \multirow[b]{2}{*}{ Casing } & \multirow[b]{2}{*}{ Instailed } & \multirow[b]{2}{*}{ Abandoned } \\
\hline & North & East & Ecreen Zone & Grnd. & Casing & & & & \\
\hline KAC 1 & 53167.0 & 42614.8 & $229.0-199.0$ & 264.0 & 266.0 & 67.0 & $4^{\prime \prime}$ PVC & $10-27-83$ & \\
\hline KAC 2 & 53255.5 & 42677.2 & $225.4-195.4$ & 256.4 & 257.5 & 62.1 & $4^{n}$ PVC & $10.31-83$ & \\
\hline KAC $\mathbf{3}$ & 53201.8 & 42723.9 & $225.8-195.8$ & 265.8 & 257.8 & 62.0 & 4" PVC & $11.02-83$ & \\
\hline KAC 4 & 53053.5 & 42676.4 & $208.0-178.0$ & 268.0 & 260.0 & 82.0 & 4" PVC & $07-12-84$ & \\
\hline KAC 5 & 63161.7 & 42716.3 & $224.3-204.3$ & 256.8 & 259.0 & 54.7 & $4^{n}$ PVC & $09-21-88$ & \\
\hline KAC 6 & 63139.9 & 42698.5 & $224.6-204.6$ & 257.1 & 259.0 & 64.4 & $4^{n}$ PVC & $09-20-88$ & \\
\hline KAC 7 & 63252.9 & 42574.6 & $223.0-203.0$ & 263.0 & 265.1 & 62.1 & $4^{n}$ PVC & 09.16 .88 & \\
\hline
\end{tabular}

\section{KCB WELL SERIES}

Description: K-Area Coal Pile Runoff Containment Basin

Bldg. Number: 189-K

Map: Figure A-4

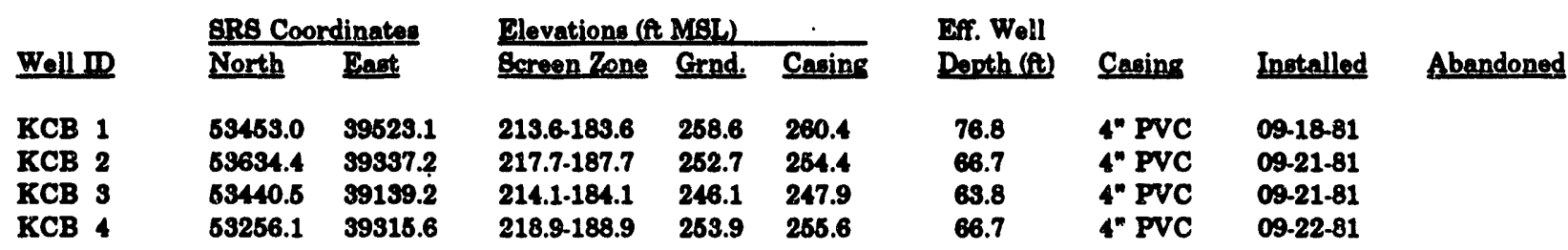

\section{KDB WELL SERIES}

Description: K-Area Disassembly Basin

Bldg. Number: 105-K

Map: Figure A-4

\begin{tabular}{|c|c|c|c|c|c|c|c|c|c|}
\hline \multirow[b]{2}{*}{ Well IP } & \multicolumn{2}{|c|}{ 8R8 Coordinates } & \multicolumn{3}{|c|}{ Elevations (ft MSL) } & \multirow{2}{*}{$\begin{array}{l}\text { Eff. Well } \\
\text { Depth (ft) }\end{array}$} & \multirow[b]{2}{*}{ Casing } & \multirow[b]{2}{*}{ Ingtalled } & \multirow[b]{2}{*}{ Abandoned } \\
\hline & North & East & Screen Zone & Grnd. & Casing & & & & \\
\hline KDB 1 & 54050.5 & 40426.9 & $205.8-184.8$ & 270.8 & 273.1 & 88.3 & 4" PVC & $08-11-86$ & \\
\hline KDB 2 & 53907.3 & 40241.4 & 203.5-182.5 & 270.6 & 273.6 & 91.0 & 4" PVC & $08-13-86$ & \\
\hline $\mathbf{K D B} 3$ & 53794.6 & 40393.7 & $205.4-184.2$ & 270.5 & 279.4 & 89.2 & 4" PVC & $08-18-86$ & \\
\hline
\end{tabular}




\section{KDT WELL SERIES}

Description: K-Area Diesel Tank

Bldg. Number: 108-1K

Map: Figure A-4

\begin{tabular}{|c|c|c|c|c|c|c|c|c|c|}
\hline \multirow[b]{2}{*}{ Well ID } & \multicolumn{2}{|c|}{ BRs Coordinates } & \multicolumn{3}{|c|}{ Elovations (ft MSL) } & \multirow{2}{*}{$\begin{array}{l}\text { Eff. Well } \\
\text { Depth (ft) }\end{array}$} & \multirow[b]{2}{*}{ Casing } & \multirow[b]{2}{*}{ Installed } & \multirow[b]{2}{*}{ Abandoned } \\
\hline & North & Fant & Screen Zone & Grnd. & Casing & & & & \\
\hline $\mathbf{K D T}$ & 54154.1 & 40380.0 & 213.7-193.7 & 270.7 & 273.0 & 79.3 & $4^{n}$ PVC & $09-28-89$ & \\
\hline
\end{tabular}

KRB WELL SERIES (see also KWL WELL SERIES)

Description: K-Area Retention Basin

Bldg. Number: $90,88 \mathrm{G}$

Map: Figure A-4

\begin{tabular}{|c|c|c|c|c|c|c|c|c|c|}
\hline \multirow[b]{2}{*}{ Well ID } & \multicolumn{2}{|c|}{ BRS Coordinater } & \multicolumn{3}{|c|}{ Hevations (ft MSL) } & \multirow{2}{*}{$\begin{array}{l}\text { Eff. Well } \\
\text { Depth (A) }\end{array}$} & \multirow[b]{2}{*}{ Casing } & \multirow[b]{2}{*}{ Installed } & \multirow[b]{2}{*}{ Abandoned } \\
\hline & North & East & Screen Zone & Grnd. & Casing & & & & \\
\hline KRB 1 & 65025.3 & 39952.1 & & 264.6 & 266.5 & & $4^{n}$ Stoel & $04-14-76$ & $05-02-91$ \\
\hline KRB 8 & 64893.6 & 40302.1 & 215.8-195.8 & 265.8 & 267.9 & 72.1 & 4" Btoel & $04-22-76$ & $06-06-91$ \\
\hline $\begin{array}{l}\text { KRB } 13 \\
\text { KRB } 14\end{array}$ & $\begin{array}{l}66344.2 \\
65566.7\end{array}$ & $\begin{array}{l}39986.6 \\
40158.5\end{array}$ & & $\begin{array}{l}281.8 \\
281.3\end{array}$ & $\begin{array}{l}289.6 \\
282.5\end{array}$ & & 4" Btool & $04-24-76$ & 05-01.91 \\
\hline KRB 15 & 55476.3 & 40669.3 & 217.6-207.6 & 267.6 & 269.6 & 62.0 & 4" Btoel & $04-22-76$ & 06-01-91 \\
\hline
\end{tabular}

Notes: There has been much confusion about the relationehip between the KRB wells and the KWL wells. It has been determined that they are eeparate well cories with different coordinates. The KRBB wells may have replaced the $\mathrm{ER}$. welle, but none of the EWL welle were officially abandoned until EWL 8 was abandoned in 1991.

\section{KRP WELL SERIES}

Description: K-Area Burning/Rubble Pit

Bldg. Number: 131-K

Map: Figure A-4

\begin{tabular}{|c|c|c|c|c|c|c|c|c|c|}
\hline \multirow[b]{2}{*}{ Well ID } & \multicolumn{2}{|c|}{ SRS Coordinates } & \multicolumn{3}{|c|}{ Flevations (ft MSL) } & \multirow{2}{*}{$\begin{array}{l}\text { Eff. Woll } \\
\text { Depth (ft) }\end{array}$} & \multirow[b]{2}{*}{ Caging } & \multirow[b]{2}{*}{ Installed } & \multirow[b]{2}{*}{ Abandoned } \\
\hline & North & Eaet & Ecreen Zone & Grnd. & Casing & & & & \\
\hline KRP 1 & 54644.0 & 42471.2 & 237.0-207.0 & 262.0 & 263.9 & 56.9 & $4^{n}$ PVC & $10-06-83$ & \\
\hline KRP 2 & 64503.6 & 42681.6 & 229.2-199.2 & 264.2 & 256.1 & 56.9 & $4^{n}$ PVC & $10-04-83$ & \\
\hline $\begin{array}{ll}\text { KRP } & 3 \\
\text { KRP } & 4\end{array}$ & $\begin{array}{l}54248.7 \\
64962.9\end{array}$ & $\begin{array}{r}42814.3 \\
42590.3\end{array}$ & $\begin{array}{l}237.6-207.6 \\
218.7 .188 .7\end{array}$ & $\begin{array}{l}252.5 \\
253.7\end{array}$ & $\begin{array}{l}264.6 \\
255.6\end{array}$ & $\begin{array}{l}47.0 \\
66.9\end{array}$ & $\begin{array}{l}\text { 4" PVC } \\
\text { 4" PVC }\end{array}$ & $\begin{array}{l}10-07-83 \\
08-07-84\end{array}$ & \\
\hline
\end{tabular}




\section{KSB WELL SERIES}

Description: K-Area Reactor Seepage Basin

Bldg. Number: 904-65G

Map: Figure A-4

\begin{tabular}{|c|c|c|c|c|c|c|c|c|c|}
\hline \multirow[b]{2}{*}{ Well DD } & \multicolumn{2}{|c|}{ 8R8 Coordinates } & \multicolumn{3}{|c|}{ Plevations (At MSL) } & \multirow{2}{*}{$\begin{array}{l}\text { Eff. Well } \\
\text { Depth (ft) }\end{array}$} & \multirow[b]{2}{*}{ Caging } & \multirow[b]{2}{*}{ Installed } & \multirow[b]{2}{*}{ Abandoned } \\
\hline & North & Eagt & Erreen Zone & Grnd. & Casing & & & & \\
\hline K8B 1 & 54044.4 & 39806.8 & $205.6-175.6$ & 265.6 & 267.4 & 91.8 & $4^{\prime \prime}$ PVC & 09.17 .84 & \\
\hline KSB 2 & 59927.6 & 39703.4 & $203.8-173.8$ & 263.8 & 265.8 & 92.0 & 4" PVC & $09-18-84$ & \\
\hline K8B 3 & 54040.2 & 39625.3 & 199.7-169.7 & 259.7 & 261.5 & 91.8 & $4^{\prime \prime}$ PVC & $09-20-84$ & \\
\hline $\begin{array}{ll}\mathrm{KSB} & 4 \\
\mathrm{~K} 8 \mathrm{~B} & 4 \mathrm{~A}\end{array}$ & $\begin{array}{l}54194.4 \\
54140.4\end{array}$ & $\begin{array}{l}39750.9 \\
39756.7\end{array}$ & $199.6-169.6$ & 259.1 & 264.1 & 94.5 & $\begin{array}{l}4^{n} \text { PVC } \\
4^{n} \text { PVC }\end{array}$ & $\begin{array}{l}09-24-84 \\
10-08-84\end{array}$ & $10-08-84$ \\
\hline
\end{tabular}

Notes: ISB 4 was never monitored because the screen was grouted.

\section{KSS WELL SERIES}

Description: K-Area Sludge Land Application Site Bldg. Number: 761-4G

Map: Figure A-4

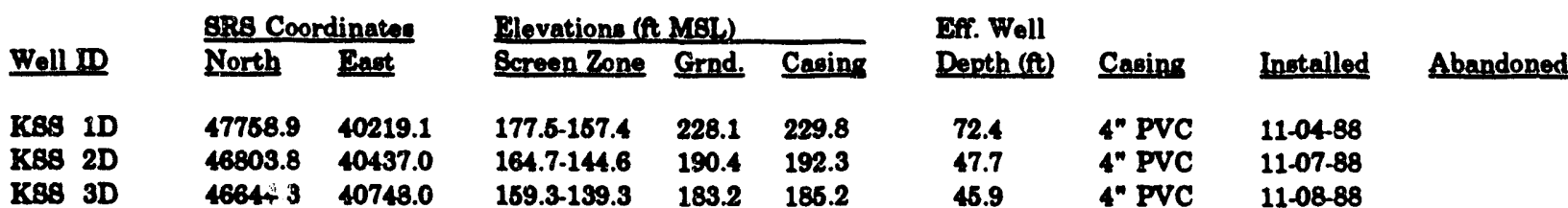

KWL WELL SERIES (see also KRB WELL SERIES)

Description: K-Area Water Level Observation Wells

Bldg. Number: 904-88G

Map: Figure A-4

\begin{tabular}{|c|c|c|c|c|c|c|c|c|c|}
\hline \multirow[b]{2}{*}{ Well ID } & \multicolumn{2}{|c|}{ SRS Coordinates } & \multicolumn{3}{|c|}{ Blevations (ft MSL) } & \multirow{2}{*}{$\begin{array}{l}\text { Eff. Well } \\
\text { Depth (ft) }\end{array}$} & \multirow[b]{2}{*}{ Casing } & \multirow[b]{2}{*}{ Installed } & \multirow[b]{2}{*}{ Abandoned } \\
\hline & North & East & Boreen Zone & Grnd. & Casing & & & & \\
\hline KWL 1 & 65027 & 39965 & & 258 & 259 & & $4^{n}$ Bteel & $09-08-63$ & \\
\hline KWL 5 & 54822 & 41755 & & 267 & 269 & & Steel & $07-23-63$ & \\
\hline KWL 8 & 54890 & 40302 & & & & & Stoel & 1963 & $11-24-75$ \\
\hline $\mathbf{K W L} 8$ & 64890 & 40302 & $220.6-200.6$ & 265.6 & & & 4" Bteel & $11-24-75$ & $05-07.91$ \\
\hline KWL 13 & 65346 & 40012 & 212.8-192.8 & 276.8 & 280.8 & 88.0 & 3.19" Stoel & $01-27-68$ & \\
\hline KWL 14 & 65559 & 40187 & & 276.7 & 279.4 & & 3.19" Bteel & $01-28-66$ & \\
\hline KWL 15 & 65467 & 40694 & & 263.1 & 265.4 & & 3.19" Btoel & $02.01-68$ & \\
\hline KWL 16 & 63992 & 39486 & $199.3-199.0$ & 259.8 & 261.8 & 62.8 & 3" Steel & $06-30-66$ & \\
\hline
\end{tabular}

Notes: There has been much confusion about the relationship between the KRBB wells and the KWL wells. It has been determined that they are eeparate well series with different coordinates. The KRB wells may have replaced the EWL wells, but none of the KWL wells were officially abandoned until KWL 8 was abandoned in 1991.

According to a well computation sheet for KWL 8, on 11-24-75 a aplit casing in the well was replaced. 


\section{LAC WELL SERIES}

Description: L-Area Acid/Caustic Basin

Bldg. Number: 904-79G

Map: Figure A-5

\begin{tabular}{|c|c|c|c|c|c|c|c|c|c|}
\hline \multirow[b]{2}{*}{ Well ID } & \multicolumn{2}{|c|}{ ERS Coordinates } & \multicolumn{3}{|c|}{ Plevations (At MSL) } & \multirow{2}{*}{$\begin{array}{l}\text { Eff. Well } \\
\text { Depth (ft) }\end{array}$} & \multirow[b]{2}{*}{ Casing } & \multirow[b]{2}{*}{ Inotalled } & \multirow[b]{2}{*}{ Abandoned } \\
\hline & North & Fast & Screen Zone & Grnd. & Casing & & & & \\
\hline $\begin{array}{ll}\text { LAC } & 1 \\
\text { LAC } & 2 \\
\text { LAC } & 3 \\
\text { LAC } & 4\end{array}$ & $\begin{array}{l}45298.8 \\
45930.4 \\
45201.9 \\
46213.1\end{array}$ & $\begin{array}{l}51318.8 \\
51270.2 \\
51186.8 \\
51270.4\end{array}$ & $\begin{array}{l}221.1-191.1 \\
223.4-193.4 \\
220.7-190.7 \\
215.3-185.3\end{array}$ & $\begin{array}{l}236.1 \\
238.4 \\
235.7 \\
235.3\end{array}$ & $\begin{array}{l}238.2 \\
240.2 \\
237.8 \\
237.1\end{array}$ & $\begin{array}{l}47.1 \\
46.8 \\
47.1 \\
51.8\end{array}$ & $\begin{array}{l}\text { 4" PVC }^{\prime \prime} \text { 4" PVC } \\
\text { 4" PVC } \\
\text { 4" PVC }\end{array}$ & $\begin{array}{l}09-30-83 \\
09-29-83 \\
10-03-83 \\
07-10-84\end{array}$ & \\
\hline
\end{tabular}

\section{LAW WELL SERIES}

Description: L-Area Research Wells

Bldg. Number: Not applicable

Map: Figure A-5

\begin{tabular}{|c|c|c|c|c|c|c|c|c|c|}
\hline \multirow{2}{*}{\multicolumn{2}{|c|}{ Well ID }} & \multicolumn{2}{|c|}{ SR8 Coordinates } & \multicolumn{3}{|c|}{ Hevations (ft MSL) } & \multirow{2}{*}{$\begin{array}{l}\text { Iff. Well } \\
\text { Depth (t) }\end{array}$} & \multirow[b]{2}{*}{ Caring } & \multirow[b]{2}{*}{ Installed } \\
\hline & & North & Pract & Ecreen Tone & Grind. & Caring & & & \\
\hline $\begin{array}{ll}\text { LAW } 1 \\
\text { LAW }\end{array}$ & $1 \mathbf{A}$ & 14565.6 & 60828.0 & (167.2-162.2) & 216.8 & 219.1 & 381.3 & & \\
\hline LAW 1 & 1C & 44662.4 & 60603.6 & (29.0. 94.0$)$ & $\begin{array}{l}216.4 \\
216.0\end{array}$ & $\begin{array}{l}218.7 \\
218.6\end{array}$ & 252.6 & & $02-16-86$ \\
\hline & 10 & 48662.0 & 60596.6 & 11.6. 6.6 & 216.6 & 218.1 & 211.6 & & $02-19-85$ \\
\hline LAW & $\mathbf{1 E}$ & 44561.2 & 60679.0 & $96.1 \cdot 90.1$ & 215.1 & 217.8 & 127.7 & ve & $02-21-85$ \\
\hline LAW & $1 \mathbf{P}$ & 41562.1 & 60567.1 & $185.9-166.9$ & 214.9 & 217.3 & 61.4 & & $02-26-85$ \\
\hline LAW & 1TD & 44664.1 & 50640.5 & (212.9-217.9) & 217.1 & 219.1 & 437.0 & $4^{n} \mathrm{Cs}$ & $02-07-85$ \\
\hline LAW & $2 A$ & 46626.5 & 49637.6 & (147.2-152.2) & 222.8 & 224.6 & 376.8 & & $03-03-85$ \\
\hline LAW & 28 & 46641.0 & 49856.5 & $(4.8-9.8)$ & 229.2 & 225.3 & 236.1 & 4" PVC & $02-01.86$ \\
\hline & $2 C$ & 46810.9 & 49638.7 & $191.2-171.2$ & 222.2 & 224.0 & 62.8 & & $02-28-86$ \\
\hline LAW & SA & 46585.8 & 52286.2 & $(169.0-164.0)$ & 248.0 & 248.5 & 412.6 & $4^{\prime \prime} \mathrm{CS}$ & $02-12.85$ \\
\hline & SB & 46600.7 & 52269.5 & 4.0. (1.0) & 246.0 & 248.4 & 249.4 & & $02-18-86$ \\
\hline LAW & SC & 46816.1 & 52272.9 & 214.9-194.9 & 246.9 & 248.0 & 63.1 & 4" PVC & $02-27.85$ \\
\hline
\end{tabular}

Notes: The Screen Zone Elevations in parenthesee are in foet below MSL.

\section{LCO WELL SERIES}

Description: L-Area Oil and Chemical Basin

Bldg. Number: 904-83G

Map: Figure A.5

\begin{tabular}{|c|c|c|c|c|c|c|c|c|c|}
\hline \multirow[b]{2}{*}{ We.lII } & \multicolumn{2}{|c|}{ 8R8 Coondinates } & \multicolumn{3}{|c|}{ Flevations (ft MST) } & \multirow{2}{*}{$\begin{array}{l}\text { Bfi. Well } \\
\text { Depth (t) }\end{array}$} & \multirow[b]{2}{*}{ Casing } & \multirow[b]{2}{*}{ Ingtalled } & \multirow[b]{2}{*}{ Abandone } \\
\hline & North & East & Ecreen Zone & Grad. & Casing & & & & \\
\hline $\begin{array}{ll}\text { LCO } & 1 \\
\text { LCO } & 2\end{array}$ & $\begin{array}{l}45198.2 \\
45317.8\end{array}$ & $\begin{array}{l}60967.7 \\
51043.4\end{array}$ & $\begin{array}{l}225.8-196.8 \\
226.6-196.6\end{array}$ & $\begin{array}{l}238.8 \\
259.6\end{array}$ & $\begin{array}{l}240.7 \\
241.6\end{array}$ & $\begin{array}{l}44.9 \\
45.0\end{array}$ & $\begin{array}{l}\text { 4" PVC } \\
\text { 4" PVC }\end{array}$ & $\begin{array}{l}11-06-81 \\
11-10-81\end{array}$ & \\
\hline
\end{tabular}

(series continued) 
LCO WELL SERIES (cont.)

\begin{tabular}{|c|c|c|c|c|c|c|c|c|c|}
\hline \multirow[b]{2}{*}{ Well ID } & \multicolumn{2}{|c|}{ SRS Coordinates } & \multicolumn{3}{|c|}{ Elevations (ft MSL) } & \multirow{2}{*}{$\begin{array}{l}\text { Eff. Well } \\
\text { Depth (ft) }\end{array}$} & \multirow[b]{2}{*}{ Caging } & \multirow[b]{2}{*}{ Installed } & \multirow[b]{2}{*}{ Abandoned } \\
\hline & North & East & Screen Zone & Grnd. & Caging & & & & \\
\hline LCO 3 & 46203.0 & 51113.2 & 226.3-196.3 & 239.3 & 241.4 & 45.1 & 4" PVC & $11-09-81$ & \\
\hline LCO 4 & 46087.4 & 51036.1 & 222.3-192.3 & 235.3 & 237.2 & 44.9 & 4" PVC & $11-05-81$ & \\
\hline
\end{tabular}

Notes: The COH Series was changed to LCO in 1985. This series is referred to as COH in Christensen and Gordon (1983).

\section{LDB WELL SERIES}

Description: L-Area Disassembly Basin

Bldg. Number: 105-L

Map: Figure A-5

\begin{tabular}{|c|c|c|c|c|c|c|c|c|}
\hline \multirow[b]{2}{*}{ Well ID } & \multicolumn{2}{|c|}{ 8R8 Coordinates } & \multicolumn{3}{|c|}{ Elevations (ft MSL) } & \multirow{2}{*}{$\begin{array}{l}\text { Eff. Well } \\
\text { Depth (ft) }\end{array}$} & \multirow[b]{2}{*}{ Casing } & \multirow[b]{2}{*}{ Installed } \\
\hline & North & East & Screen Zone & Grnd. & Casing & & & \\
\hline $\begin{array}{ll}D B & 1 \\
0\end{array}$ & 46067.3 & 60530.6 & 215.0 .185 .0 & 250.5 & 252.9 & 67.9 & 4" PVC & 09-24-85 \\
\hline
\end{tabular}

Notes: LDB 3 was not drilled.

\section{LFW WELL SERIES}

Description: Sanitary Landfill

Bldg. Number: 740-G

Map: Figure A-27

\begin{tabular}{|c|c|c|c|c|c|c|c|c|c|}
\hline \multirow[b]{2}{*}{ Well ID } & \multicolumn{2}{|c|}{ BR8 Coordinates } & \multicolumn{3}{|c|}{ Blevations (A MSL) } & \multirow{2}{*}{$\begin{array}{l}\text { Bff. Well } \\
\text { Depth (At) }\end{array}$} & \multirow[b]{2}{*}{ Caging } & \multirow[b]{2}{*}{ Installed } & \multirow[b]{2}{*}{ Abandoned } \\
\hline & North & East & Screen Zone & Grnd. & Casing & & & & \\
\hline LFW 1 & 84691.8 & 45852.3 & $162.0-142.0$ & 177.0 & 179.6 & 37.6 & 4" Btoel & $02-10-75$ & $11-24-86$ \\
\hline LNW 2 & 84579.8 & 45498.2 & $163.6-143.6$ & 173.6 & 176.7 & 33.1 & 4" Steel & $02-11-75$ & $12-05-86$ \\
\hline LFW 9 & 84554.0 & 46346.8 & $167.0-137.0$ & 172.0 & 173.9 & 36.9 & 4" Stoel & $03-14-75$ & $12-04-86$ \\
\hline LFW 4 & 84856.8 & 45103.9 & $162.0-142.0$ & 177.0 & 178.7 & 36.7 & $4^{n}$ Steel & $03-17.75$ & $12.02-86$ \\
\hline LFW 5 & 85268.0 & 45629.1 & 169.1-149.1 & 179.4 & 181.4 & 32.3 & $4^{*}$ Bteel & $06-22-78$ & $12-01-86$ \\
\hline LPW 6 & 84537.8 & 45241.2 & $160.4-141.1$ & 170.2 & 171.7 & 30.6 & $4^{n}$ Btoel & $02-02-81$ & \\
\hline LWW 7 & 84310.3 & 45318.9 & $169.8-140.6$ & 169.6 & 171.2 & 30.7 & $4^{\prime \prime}$ Btoel & $01-30-81$ & \\
\hline LFW 8 & 84032.6 & 45416.3 & 169.2-139.9 & 169.0 & 170.5 & 30.6 & $4^{n}$ Btoel & $01-29-81$ & \\
\hline LNW 9 & 84074.8 & 45802.8 & $163.1-143.1$ & 172.9 & 174.9 & 31.8 & $4^{\prime \prime}$ Btoel & $01-28-81$ & $11-26-86$ \\
\hline LFW 10 & 84360.0 & 45916.0 & $165.2-145.2$ & 176.0 & 177.0 & 31.8 & $4^{n}$ Btoel & $01.27-81$ & $01.01 .84^{*}$ \\
\hline LFW 10A & 84369.6 & 45935.6 & 159.2-129.2 & 174.2 & 176.5 & 46.3 & 4" PVC & $01-13-84$ & \\
\hline LNW 16 & 84748.9 & 45852.6 & $161.2-131.2$ & 177.2 & 178.8 & 47.6 & $4^{n}$ PVC & $08-26-81$ & \\
\hline LWW 17 & 84602.8 & 45607.3 & $158.5-128.5$ & 176.5 & 177.8 & 49.3 & $4^{n} \mathrm{PVC}$ & $08-28-81$ & \\
\hline LFW 18 & 84577.3 & 45459.4 & $160.1 \cdot 130.1$ & 174.1 & 175.0 & 44.9 & $4^{n}$ PVC & $08-27.81$ & \\
\hline LFW 19 & 84817.2 & 45135.4 & $160.0-130.0$ & 175.0 & 176.7 & 46.7 & 4" PVC & $08-31.81$ & \\
\hline LFW 20 & 85262.6 & 45582.9 & $165.0-135.0$ & 179.0 & 180.5 & 45.5 & $4^{n}$ PVC & $08-25-81$ & \\
\hline LFW 21 & 84178.3 & 46149.4 & $158.9-128.9$ & 173.9 & 175.6 & 46.7 & 4" PVC & $01-11.84$ & \\
\hline LFW 22 & 84223.6 & 46325.2 & $152.4-122.4$ & 172.4 & 174.2 & 61.8 & 4" PVC & $01-12-84$ & \\
\hline LFW 23 & 84251.3 & 46456.1 & $155.1-125.1$ & 170.1 & 171.8 & 46.7 & $4^{\prime \prime} \mathrm{PVC}$ & 01.12 .84 & \\
\hline
\end{tabular}

(series continued) 
LFW WELL SERIES (cont.)

\begin{tabular}{|c|c|c|c|c|c|c|c|c|c|}
\hline \multirow[b]{2}{*}{ Well ID } & \multicolumn{2}{|c|}{ BRS Coordinatog } & \multicolumn{3}{|c|}{ Elevations (ft MSI) } & Effi. Well & \multirow[b]{2}{*}{ Casing } & \multirow[b]{2}{*}{ Installed } & \multirow[b]{2}{*}{ Abandoned } \\
\hline & North & East & Bcreen Zone & Grnd. & Casina & Depth (ft) & & & \\
\hline LNW 24 & 84644.2 & 46620.8 & $154.6-124.6$ & 169.6 & 171.3 & 46.8 & $4^{\circ}$ PVC & $01-13-84$ & \\
\hline $\begin{array}{l}\text { LFW } 25 \\
\text { LFW } 26\end{array}$ & $\begin{array}{l}84967.2 \\
86654.6\end{array}$ & $\begin{array}{r}46425.7 \\
46639.8\end{array}$ & $\begin{array}{l}163.2-123.2 \\
164.2-143.2\end{array}$ & $\begin{array}{l}173.2 \\
184.2\end{array}$ & $\begin{array}{l}174.7 \\
186.6\end{array}$ & $\begin{array}{l}61.5 \\
43.3\end{array}$ & $\begin{array}{l}\text { 4" PVC }^{n} \text { PVC } \\
4^{n} \text { PVC }\end{array}$ & $\begin{array}{l}01-13-84 \\
10-29-86\end{array}$ & \\
\hline LNW 27 & 85899.1 & 46596.1 & $163.9-142.9$ & 186.9 & 189.2 & 46.3 & $4^{n}$ PVC & $10-30-86$ & \\
\hline LNW 28 & 86079.6 & 46556.3 & 162.1-141.1 & 190.1 & 192.4 & 61.3 & 4" PVC & $10-28-86$ & \\
\hline LNW 29 & 86372.7 & 46509.9 & $164.9-149.9$ & 192.9 & 196.3 & 51.4 & 4" PVC & $10-28-86$ & \\
\hline $\mathrm{LNW} 30$ & 86318.4 & 45170.9 & $162.7-141.7$ & 207.7 & 210.0 & 68.3 & $4^{\circ} \mathrm{PVC}$ & $12-31-86$ & \\
\hline LIW 31 & 86862.2 & 44869.0 & $166.0-145.0$ & 227.0 & 229.3 & 84.3 & 4" PVC & $10-27-86$ & \\
\hline LFW 92 & 85886.8 & 44936.9 & $166.3-144.3$ & 221.3 & 223.7 & 79.4 & $4^{n}$ PVC & $10-24-86$ & \\
\hline LFW 39 & 85633.8 & 4973.0 & 165.4144 .4 & 211.4 & 213.7 & 69.3 & $4^{n}$ PVC & $10-23-86$ & \\
\hline LFW 34 & 86409.5 & 45016.9 & $164.7-143.7$ & 198.7 & 201.0 & 67.3 & $4^{n}$ PVC & $10-22-86$ & \\
\hline LFW 36 & 85237.4 & 46378.8 & 164.4143 .4 & 181.4 & 183.7 & 40.3 & $4^{n}$ PVC & $10-21-86$ & \\
\hline LFW 36 & 83535.6 & 45582.3 & $151.2-130.3$ & 168.3 & 170.4 & 40.1 & 4" PVC & $11-05-86$ & \\
\hline LFW 37 & 89113.2 & 46667.7 & $160.8-129.8$ & 167.8 & 169.9 & 40.1 & 4" PVC & $11-04-86$ & \\
\hline LFW 38 & 83172.3 & 46018.6 & 151.6-130.6 & 168.6 & 170.3 & 39.8 & 4" PVC & $11-03-86$ & \\
\hline LFW 99 & 89213.1 & 46218.6 & $162.2-131.2$ & 169.2 & 171.4 & 40.2 & $4^{n}$ PVC & $11-08-88$ & \\
\hline LFW 40 & 85248.8 & 46996.1 & $162.2-131.2$ & 169.2 & 171.0 & 39.8 & $4^{\prime \prime}$ PVC & $10-31-86$ & \\
\hline INW 41 & 89904.9 & 46626.9 & $161.3-130.3$ & 168.3 & 170.6 & 40.2 & $4^{*}$ PVC & $10-31.86$ & \\
\hline LFW 42 & 83776.2 & 16582.9 & $151.2-190.2$ & 168.2 & 170.1 & 39.9 & $4^{n}$ PVC & $10-30-86$ & \\
\hline LFW 43B & 88469.2 & 45240.6 & 99.9- 89.9 & 200.5 & 203.0 & 113.1 & $4^{n}$ PVC & $06-21.91$ & \\
\hline LFW 43C & 86480.6 & 45234.9 & 138.1-128.1 & 200.1 & 202.6 & 74.6 & $4^{*}$ PVC & $08-25-91$ & \\
\hline LFW 43D & 86443.2 & 45244.6 & $170.4-150.4$ & 200.4 & 202.9 & 62.5 & $4^{n}$ PVC & $06-2491$ & \\
\hline LFW $4 D$ & 84524.4 & 46022.6 & $159.3-139.5$ & 168.3 & 170.3 & 30.8 & $4^{n}$ PVC & $06-29-91$ & \\
\hline LFW 46D & 84217.8 & 45142.0 & $154.7-194.7$ & 164.4 & 168.3 & 31.6 & 4" PVC & $06-16-91$ & \\
\hline LWW 46D & 84054.0 & 46162.8 & $167.1-137.3$ & 163.1 & 165.1 & 27.8 & $4^{n}$ PVC & $05-29-91$ & \\
\hline LWW 47C & 83843.9 & 46178.6 & $116.8-105.7$ & 169.3 & 161.4 & 66.7 & 4" PVC & $00-29-91$ & \\
\hline LFW 4TD & 83869.3 & 46167.9 & 154.7.134.9 & 169.7 & 161.7 & 26.8 & $4^{\prime \prime}$ PVC & $05-23-91$ & \\
\hline LFW 48C & 88877.0 & 45430.3 & $118.2-108.2$ & 167.2 & 169.8 & 61.1 & $4^{n}$ PVC & $05-28-91$ & \\
\hline LNW 48D & 85877.6 & 46449.7 & $165.0-134.9$ & 167.5 & 169.6 & 94.6 & $4^{n}$ PVC & $06-28-91$ & \\
\hline LFW 65C & 85613.2 & 46208.9 & 103.9-93.9 & 163.9 & 166.4 & 62.6 & $4^{n}$ PVC & $05-14-81$ & \\
\hline LFW 66D & 89601.3 & 46189.3 & $141.4-121.2$ & 163.4 & 166.4 & 34.2 & $4^{*}$ PVC & $05-16-91$ & \\
\hline LFW 66D & 89998.0 & 46308.6 & $161.4-131.3$ & 165.9 & 168.1 & 26.8 & $4^{n}$ PVC & $05-22-91$ & \\
\hline LFW $57 B$ & 89196.7 & 46440.6 & $78.4-68.4$ & 169.4 & 165.4 & 97.0 & 4" PVC & $06-11-91$ & \\
\hline LWW 67c & 89200.1 & 46411.1 & $117.9-107.8$ & 162.9 & 165.0 & 67.2 & $4^{\star}$ PVC & $06-21-91$ & \\
\hline IFW $57 D$ & 89190.2 & 46417.4 & $160.4-190.6$ & 162.9 & 164.8 & 34.2 & $4^{n}$ PVC & $0 .-21.91$ & \\
\hline LFW 68D & 82940.6 & 46700.2 & $147.6-127.6$ & 165.6 & 167.6 & 40.1 & 4" PVC & 05.21 .91 & \\
\hline LFW 59B & 85027.1 & 46047.4 & 75.6- 65.6 & 166.6 & 168.1 & 102.6 & $4^{\circ}$ PVC & $08-13-91$ & \\
\hline LFW 59C & 85011.0 & 46052.0 & 109.8. 99.8 & 164.8 & 167.3 & 67.5 & 4" PVC & $06-18-91$ & \\
\hline LTW 690 & 89000.1 & 46056.1 & 149.1-129.1 & 165.1 & 167.6 & 38.6 & $4^{*}$ PVC & $08-18-91$ & \\
\hline LFW GOD & 82531.6 & 45722.3 & 143.2.123.2 & 164.6 & 167.1 & 33.9 & $4^{n}$ PVC & $08-18-91$ & \\
\hline LFW 61C & 83084.4 & 46489.6 & $120.8-110.7$ & 168.8 & 168.3 & 67.6 & 4" PVC & $05-16-91$ & \\
\hline WW 610 & 85089.1 & 16471.1 & 160.4130 .3 & 168.4 & 168.3 & $\mathbf{3 8 . 0}$ & $4 "$ PVC & $00-20-91$ & \\
\hline LWW $62 B$ & 89001.2 & 46916.5 & $72.4-62.4$ & 162.4 & 164.9 & 102.6 & $4^{\prime \prime}$ PVC & $06-11-91$ & \\
\hline LFW 62C & 83012.7 & 45906.7 & 118.0 .108 .0 & 163.0 & 166.5 & 67.6 & 4" PVC & $06-11-91$ & \\
\hline LFW 62D & 82991.6 & 46922.9 & $147.6-127.6$ & 162.6 & 164.8 & 37.2 & 4" PVC & $08-06-91$ & \\
\hline
\end{tabular}

Notes: Zeigler, Lawrimore, and O'Rear (1985) atute that wells IWW 6 through 10 have PVC casings. However, the Drilling Requeste, Boring Logs, and Abandonment Data etate that these welle heve PVC ecreens and galvanizod eteel casinge.

IWW 1 has a steel casing for approximately the first $5 \mathrm{ft}$, then PVC casing and screen. 


\section{LRP WELL SERIES}

Description: L-Area Burning/Rubble Pit

Bldg. Number: 131-L

Map: Figure A-5

\begin{tabular}{|c|c|c|c|c|c|c|c|c|c|}
\hline \multirow[b]{2}{*}{ Well ID } & \multicolumn{2}{|c|}{ SRS Coordinates } & \multicolumn{3}{|c|}{ Elevations (At MSL) } & \multirow{2}{*}{$\begin{array}{l}\text { Eff. Well } \\
\text { Depth (t) }\end{array}$} & \multirow[b]{2}{*}{ Casing } & \multirow[b]{2}{*}{ Installed } & \multirow[b]{2}{*}{ Abandoned } \\
\hline & North & East & Bcreen Zone & Grnd. & Casing & & & & \\
\hline LRP 1 & 48548.6 & 49128.7 & 215.8.185.8 & 250.8 & 252.9 & 67.1 & 4" PVC & $09.27-83$ & \\
\hline LRP 2 & 48352.9 & 49214.4 & $214.7-184.7$ & 254.7 & 256.7 & 72.0 & 4" PVC & $10-26-83$ & \\
\hline LRP 3 & 48333.6 & 49057.7 & $221.4-191.4$ & 256.4 & 258.2 & 66.8 & $4^{n}$ PVC & $09-28-89$ & \\
\hline LRP 4 & 48440.2 & 48964.7 & 203.3-173.3 & 253.3 & 255.6 & 82.3 & $4^{n}$ PVC & $07-09-84$ & \\
\hline
\end{tabular}

Notes: LRP 2 was first drilled 9-23-83 but was located over $6 \mathrm{ft}$ of concrete pipe. The well was redrilled 10-26-83, $1 \mathrm{ft}$ from the original site.

\section{LSB WELL SERIES}

Description: L-Area Reactor Seepage Basin

Bldg. Number: 904-64G

Map: Figure A.5

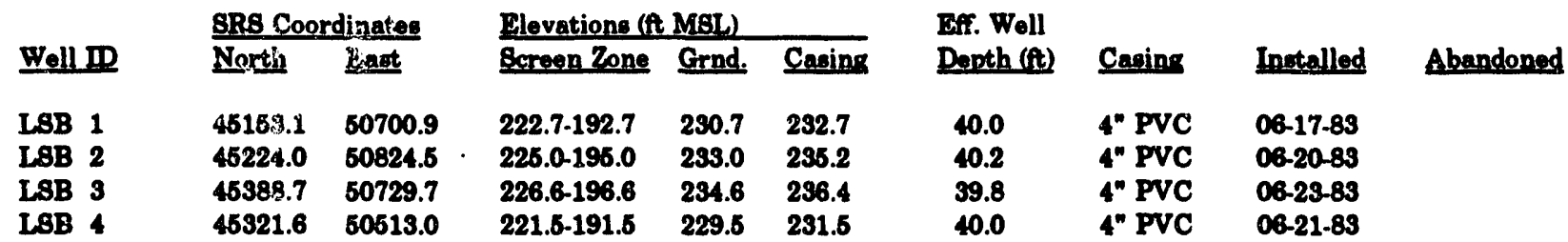

M 12 WELL SERIES (

\section{MCB WELL SERIES}

Description: Miscellaneous Chemical Basin

Bldg. Number: 731-5A

Map: Figure A-17

\begin{tabular}{|c|c|c|c|c|c|c|c|c|c|}
\hline \multirow[b]{2}{*}{ Well ID } & \multicolumn{2}{|c|}{ BRs Coordinates } & \multicolumn{3}{|c|}{ Dlevations (ft MSL) } & \multirow{2}{*}{$\begin{array}{l}\text { Efi. Well } \\
\text { Depth (ft) }\end{array}$} & \multirow[b]{2}{*}{ Carins } & \multirow[b]{2}{*}{ Installed } & \multirow[b]{2}{*}{ Abandoned } \\
\hline & North & East & Bcreen Zone & Grnd. & Caning & & & & \\
\hline MCB 2 & 97012.6 & 45129.0 & 225.9-205.9 & 326.1 & 328.4 & 122.5 & 4" PVC & $04-13-87$ & \\
\hline MCB 4 & 97532.5 & 44705.1 & 229.6-208.6 & 348.2 & 350.4 & 141.8 & 4" PVC & $04-02-87$ & \\
\hline MCB 6 & 97335.6 & 44863.9 & 226.3-206.3 & 337.7 & 399.6 & 133.3 & $4^{\prime \prime}$ PVC & $04-14-87$ & \\
\hline MCB $6 \mathrm{C}$ & 97315.1 & 44862.8 & $161.2-166.2$ & 337.2 & 339.1 & 182.9 & 4" PVC & $07.10-89$ & \\
\hline MCB 6 & 97425.7 & 45214.0 & 219.7-199.7 & 329.9 & 392.1 & 132.4 & 4" PVC & 08.10 .87 & \\
\hline MCB 6C & 97413.1 & 46207.7 & $170.0-165.0$ & 930.0 & 332.1 & 167.1 & 4" PVC & $06-21.89$ & \\
\hline $\mathrm{MCB} 7 \mathrm{C}$ & 97139.9 & 44870.6 & $160.7-155.7$ & 335.7 & 337.7 & 182.0 & $4^{*} \mathrm{PVC}$ & 07.17 .89 & \\
\hline
\end{tabular}

(eeries continued) 
MCB WELL SERIES (cont.)

\begin{tabular}{|c|c|c|c|c|c|c|c|c|c|}
\hline & SRS Co & dinates & Plevations ( & MEI) & & Eff. Well & & & \\
\hline Well ID & North & Fast & Ecreen Zone & Grnd. & Caring & Depth (ft) & Casing & Installed & Abandoned \\
\hline $\begin{array}{l}\text { MCB } 80 \\
\text { MCB } 9 D\end{array}$ & $\begin{array}{l}97180.6 \\
97606.8\end{array}$ & $\begin{array}{l}44769.9 \\
44858.8\end{array}$ & $\begin{array}{l}225.7-205.7 \\
226.2-206.2\end{array}$ & $\begin{array}{l}397.4 \\
399.1\end{array}$ & $\begin{array}{l}340.7 \\
342.9\end{array}$ & $\begin{array}{l}135.0 \\
136.7\end{array}$ & $\begin{array}{l}2^{n} \text { PVC } \\
2^{n} \text { PVC }\end{array}$ & $\begin{array}{l}07-07-89 \\
07-07-89\end{array}$ & \\
\hline
\end{tabular}

Notes: MCB 8D and 9D are piezometere.

\section{MGA WELL SERIES}

Description: Series A, Monitoring Grid Wells for Burial Grounds Bldg. Number: Old Burial Ground, 643-E

Map: Figures A-8, A-10, and A-12

\begin{tabular}{|c|c|c|c|c|c|c|c|c|c|}
\hline \multirow[b]{2}{*}{ Well ID } & \multicolumn{2}{|c|}{ BRs Coordinates } & \multicolumn{3}{|c|}{ Flevations (ft MSI) } & \multirow{2}{*}{$\begin{array}{l}\text { Eff. Well } \\
\text { Depth (t) }\end{array}$} & \multirow[b]{2}{*}{ Casing } & \multirow[b]{2}{*}{ Inotalled } & \multirow[b]{2}{*}{ Abandoned } \\
\hline & North & East & Ecreen Zone & Grnd. & Caging & & & & \\
\hline MGA 1 & 76003.6 & 66100.1 & $242.5-222.6$ & 280.5 & 282.6 & 60.0 & $4^{\prime \prime}$ Steol & $02-18-76$ & \\
\hline MGA 3 & 76893.1 & 65264.0 & $242.6-222.6$ & 281.6 & 283.5 & 61.0 & $4^{\prime \prime}$ Etreel & $02-19-76$ & \\
\hline MGA 5 & 76766.8 & 65417.0 & $244.3-224.3$ & 283.8 & 286.3 & 62.0 & $4^{\prime \prime}$ Stoel & $04-15-76$ & \\
\hline MGA 7 & 76644.0 & 66571.9 & $249.6-228.6$ & 288.1 & 286.6 & 62.0 & 4" Stoel & $04-12-78$ & \\
\hline MGA 9 & 76529.8 & 66732.6 & 243.9-223.9 & 288.4 & 288.9 & 68.0 & $4^{\infty}$ Otcel & $04-07.76$ & \\
\hline MGA 11 & 75409.9 & 65891.2 & $246.4-228.4$ & 293.9 & 296.4 & 70.0 & 4" PVC & $01.02-73$ & \\
\hline MGA 19 & 74927.0 & 86527.0 & $250.2-250.2$ & 281.7 & 284.2 & 84.0 & 4" PVC & $10-09-72$ & \\
\hline MGA 21 & 74811.6 & 68887.2 & 251.2-231.2 & 285.7 & 288.2 & 57.0 & 4" PVC & $10-17.72$ & \\
\hline MGA 28 & 74688.6 & 68844.5 & 249.7-229.7 & 288.2 & 285.7 & 66.0 & $4^{\circ}$ PVC & 10.12-72 & \\
\hline MGA 32 & 74144.8 & 67665.0 & $252.6-232.6$ & 284.1 & 286.6 & 64.0 & 4" PVC & $11-06-72$ & \\
\hline & 74023.6 & 57728.9 & 254.7-234.7 & 290.2 & 292.7 & 68.0 & & 11.07 .72 & \\
\hline MGA 38 & 79904.0 & 57891.6 & 256.3-256.3 & 298.2 & 298.3 & 62.0 & 4" PVC & $11-08-72$ & \\
\hline
\end{tabular}

Notes: The MGA Beries is one of the Grid Beries. The other Grid Series are MCC, MGE, MGG, and MCI

MCA 9 was originally inatalled 2-06-73, but, according to a nots on the Boring Log, the well was dectroyed in early 1974 when beavy equipment ran over it.

\section{MGC WELL SERIES}

Description: Series C, Monitoring Grid Wells for Burial Grounds Bldg. Number: Old Burial Ground, 643-E

Map: Figures A-8, A-10, and A-12

\begin{tabular}{|c|c|c|c|c|c|c|c|c|c|}
\hline \multirow[b]{2}{*}{ Well ID } & \multicolumn{2}{|c|}{ SIs Coordinates } & \multicolumn{3}{|c|}{ Mevations (ft MSI) } & \multirow{2}{*}{$\begin{array}{l}\text { Bef. Well } \\
\text { Depth (ft) }\end{array}$} & \multirow[b]{2}{*}{ Casing } & \multirow[b]{2}{*}{ Ingtalled } & \multirow[b]{2}{*}{ Abandoned } \\
\hline & North & East & Screen Zone & Grnd. & Casing & & & & \\
\hline MGC 1 & 75849.0 & 54978.2 & 238.8-218.8 & 276.8 & 278.8 & 60.0 & $4^{n}$ Bteel & $02-06-76$ & \\
\hline MGC 3 & 75733.0 & 56139.7 & $240.7-220.7$ & 279.4 & 281.7 & 61.0 & 4" Btoel & $02-05-76$ & \\
\hline MGC 6 & 75606.6 & 55293.0 & $242.0-222.0$ & 281.6 & 284.0 & 62.0 & $4^{\prime \prime}$ Bteol & 0209.78 & \\
\hline MGC 7 & 76502.4 & 56465.0 & $242.6-222.6$ & 282.4 & 284.6 & 62.0 & $4^{\prime \prime}$ Eteel & $02-10.76$ & \\
\hline MGC 9 & 76972.1 & 85610.7 & 239.1-219.1 & 282.3 & 284.1 & 66.0 & $4^{n}$ PVC & $01-04-73$ & \\
\hline MGC 11 & 75252.3 & 56770.7 & 241.2-221.2 & 289.2 & 291.2 & 70.0 & $4^{\prime \prime}$ PVC & $03-1473$ & \\
\hline MGC 13 & 75132.7 & 56931.4 & $247.5-227.5$ & 290.0 & 292.5 & 65.0 & 4" PVC & 02.06 .73 & \\
\hline
\end{tabular}

(weries continued) 
MGC WELL SERIES (cont.)

\begin{tabular}{|c|c|c|c|c|c|c|c|c|c|}
\hline \multirow[b]{2}{*}{ Well D } & \multicolumn{2}{|c|}{ SR8 Coordinates } & \multicolumn{3}{|c|}{ Elevations (ft MSL) } & \multirow{2}{*}{$\begin{array}{l}\text { Eff. Well } \\
\text { Depth (ft) }\end{array}$} & \multirow[b]{2}{*}{ Casing } & \multirow[b]{2}{*}{ Installed } & \multirow[b]{2}{*}{ Abandoned } \\
\hline & North & East & Screen Zone & Grnd. & Casing & & & & \\
\hline MGC 15 & 75008.6 & 56089.7 & $244.6-224.6$ & 288.7 & 289.6 & 65.0 & $4^{*}$ PVC & 02.06 .73 & \\
\hline MGC 17 & 74887.1 & 56251.6 & 249.7-229.7 & 282.2 & 284.7 & 55.0 & $4^{\prime \prime}$ PVC & $05.23-73$ & \\
\hline MGC 19 & 74770.1 & 56408.7 & 236.6-232.6 & 284.6 & 286.6 & 54.0 & $4^{\prime \prime}$ PVC & $04-17.72$ & \\
\hline MGC 21 & 74649.9 & 56566.5 & $250.4-230.4$ & 284.9 & 287.4 & 57.0 & $4^{n}$ PVC & $10-18-72$ & \\
\hline MGC 23 & 74528.3 & 56726.6 & 251.7.231.7 & 283.9 & 287.7 & 56.0 & 4" PVC & 11.16 .72 & \\
\hline MGC 30 & 74099.3 & 67292.3 & 251.7-231.7 & 280.2 & 282.7 & 51.0 & $4^{n}$ PVC & $11-08.72$ & \\
\hline MGC 32 & 73982.1 & 57448.8 & $263.0-243.0$ & 287.0 & 298.0 & 55.0 & 4" PVC & $11.08-72$ & \\
\hline MGC 34 & 73859.9 & 57612.0 & 256.5-236.5 & 289.0 & 291.6 & 55.0 & $4^{n}$ PVC & 11-13-72 & \\
\hline MGC 96 & 73738.9 & 57776.0 & 256.6-236.6 & 294.4 & 296.6 & 60.0 & $4^{\prime \prime}$ PVC & $11-13-72$ & \\
\hline
\end{tabular}

Notes: The MGC Series is one of the Grid Beries. The other Grid Series are MGA, MGE, MGG, and MGI

\section{MGE WELL SERIES}

Description: Series E, Monitoring Grid Wells for Burial Grounds Bldg. Number: Old Burial Ground, 643-E

Map: Figures A-8, A-10, and A-12

\begin{tabular}{|c|c|c|c|c|c|c|c|c|c|}
\hline \multirow[b]{2}{*}{ Well ID } & \multicolumn{2}{|c|}{ BRs Coordinates } & \multicolumn{3}{|c|}{ Elevations (ft MBL) } & \multirow{2}{*}{$\begin{array}{l}\text { Eff. Woll } \\
\text { Depth (At) }\end{array}$} & \multirow[b]{2}{*}{ Casing } & \multirow[b]{2}{*}{ Inotalled } & \multirow[b]{2}{*}{ Abandoned } \\
\hline & North & Eact & Screen Zone & Grnd. & Casing & & & & \\
\hline MGE 1 & 76698.1 & 54857.4 & 234.7-214.7 & 272.2 & 274.7 & 60.0 & $4^{n}$ Btoel & $04-13-76$ & \\
\hline MGE 3 & 76573.9 & 66016.9 & 236.9-216.9 & 275.4 & 277.9 & 62.0 & 4" Btreel & $04-14-76$ & \\
\hline MGE 5 & 75458.8 & 66174.7 & $240.7-220.7$ & 279.8 & 282.7 & 62.0 & 4" Btoel & $02-11-77$ & \\
\hline MGE 7 & 75393.0 & 65331.3 & $240.1-220.1$ & 279.5 & 282.1 & 62.0 & $4^{n}$ Streel & $02-20.76$ & \\
\hline MGE 9 & 76216.1 & 65489.4 & $240.6-220.6$ & 281.1 & 283.6 & 63.0 & $4^{\prime \prime}$ PVC & $02-16-73$ & \\
\hline MGE 13 & 74971.0 & 56809.9 & 243.4-223.4 & 285.9 & 288.4 & 65.0 & 4" PVC & $02-16-73$ & \\
\hline MGE 15 & 74848.8 & 56970.6 & $241.0-221.0$ & 282.5 & 285.0 & 64.0 & 4" PVC & $02-19-73$ & \\
\hline MGE 17 & 74731.6 & 66127.3 & 246.1-226.1 & 283.6 & 286.1 & 60.0 & $4^{\prime \prime}$ PVC & $02-09-72$ & \\
\hline MGE 19 & 74608.5 & 56287.6 & 246.9-226.9 & 281.4 & 288.9 & 67.0 & $4^{*}$ PVC & $02-16-72$ & \\
\hline MGE 21 & 74487.8 & 58446.2 & $250.0-230.0$ & 282.9 & 285.0 & 55.0 & $4^{\prime \prime}$ PVC & $04-27.72$ & \\
\hline MGE 23 & 74369.3 & 56609.2 & $249.4-229.4$ & 279.9 & 282.4 & 63.0 & $4^{\prime \prime}$ PVC & $04-18-72$ & \\
\hline MGE 30 & 73935.8 & 57175.4 & 251.2-231.2 & 280.3 & 282.2 & 51.0 & $4^{n}$ PVC & $01-05-72$ & \\
\hline MGD 32 & 73819 & 67332.6 & $254.8-234.8$ & 285.3 & 287.8 & 63.0 & $4^{n}$ PVC & $01-20-72$ & \\
\hline MGE 34 & 73695 & 57495.1 & $259.4-239.4$ & 292.2 & 294.4 & 65.0 & $4^{n}$ PVC & $06-06-72$ & \\
\hline MGE 36 & 73573 & 57657.3 & 256.7-236.7 & 293.2 & 295.7 & 59.0 & $4^{n}$ PVC & $06-06-72$ & \\
\hline
\end{tabular}

Notes: The MGE Beries is one of the Grid Beries. The other Grid Beries are MGA, MGC, MGG, and MGI 


\section{MGG WELL SERIES}

Description: Series G, Monitoring Grid Wells for Burial Grounds

Bldg. Number: Old Burial Ground, 643-E

Map: Figures A-8, A-10, and A-12

\begin{tabular}{|c|c|c|c|c|c|c|c|c|}
\hline \multirow[b]{2}{*}{ Well ID } & \multicolumn{2}{|c|}{ gRS Coordinates } & \multicolumn{3}{|c|}{ Plevations (ft MSI) } & \multirow{2}{*}{$\begin{array}{l}\text { Eff. Well } \\
\text { Depth (ft) }\end{array}$} & \multirow[b]{2}{*}{ Caging } & \multirow[b]{2}{*}{ Installed } \\
\hline & North & Eant & Screen Zone & Grnd. & Caring & & & \\
\hline MGG 1 & 76549 & 54799.6 & $237.0-217.0$ & 276.2 & 277.0 & 60.0 & $4^{n}$ Steel & $02-17-76$ \\
\hline MGG 9 & 76410 & 54888.3 & 246.8-226.8 & 286.8 & 288.8 & 62.0 & $4^{n}$ Steel & 02-12-76 \\
\hline MGG 5 & 75303 & 65058.6 & $241.8-221.8$ & 281.6 & 283.8 & 62.0 & 4" Bteel & 02-13-76 \\
\hline MGG 7 & 75172 & 66208.6 & 238.4-218.4 & 279.2 & 280.4 & 62.0 & $4^{n}$ Steel & $02-23-76$ \\
\hline MGG 9 & 75071 & 65377.6 & 244.2-224.2 & 281.2 & 283.2 & 69.0 & $4^{n}$ Steel & $03-02-76$ \\
\hline MGG 13 & 74821 & 65690.9 & $246.3-225.3$ & 283.8 & 288.3 & 61.0 & 4" PVC & $08-30-74$ \\
\hline MGG 13CL & 74811 & 66690.9 & & & & & & $04-07.72$ \\
\hline MGG 16 & 74699 & 65861.5 & $245.0-225.0$ & 282.3 & 284.0 & 69.0 & $4^{n}$ PVC & $04-07.72$ \\
\hline MGG 17 & 74578 & 66011.7 & 248.1-228.1 & 279.6 & 282.1 & 64.0 & 4" PVC & $04-10-72$ \\
\hline MGG 19 & 74466 & 66174.9 & $248.0-228.0$ & 278.0 & 280.0 & 52.0 & 4" PVC & $04-10.72$ \\
\hline MGG 21 & 74394 & 66393.1 & 248.6-228.6 & 277.1 & 279.6 & 61.0 & $4^{\prime \prime}$ PVC & 02.27 .73 \\
\hline MGG 21A & 74394 & 56939.1 & & & & & & \\
\hline MGG 21B & 74334 & 66393.1 & & & & & & \\
\hline MGG 21P & 74394 & 68393.1 & & & & & & \\
\hline MGG 23 & 74214 & 56491.8 & $248.8-228.8$ & 276.1 & 277.8 & 49.0 & 4" PVC & $02-27.73$ \\
\hline MGG 28 & 79905 & 66895.4 & 252.6-232.6 & 274.3 & 276.5 & 44.0 & $4^{n}$ PVC & $08-27-74$ \\
\hline MGG 28CL & 79905 & 66886.4 & & & & & & 10-26-72 \\
\hline MGG SO & 79781 & 57059.5 & $255.0-235.0$ & 277.6 & 280.0 & 45.0 & $4^{n}$ PVC & $10-25-72$ \\
\hline MGG 32 & 79659 & 57218.0 & 266.3-236.3 & 283.8 & 286.3 & 60.0 & $4^{\circ} \mathrm{PVC}$ & 08-02-72 \\
\hline MGG 34 & 73586 & 57379.7 & 256.6-235.6 & 288.1 & 290.6 & 55.0 & 4" PVC & 06-08-72 \\
\hline MGG 36 & 73413 & 67641.7 & 253.2-233.2 & 290.6 & 291.2 & 68.0 & 4" PVC & $08-08-72$ \\
\hline
\end{tabular}

08-20.74

08-20.74

Notes: The MGG Beries is one of the Grid Beries. The other Grid Beries are MGA, MGC, MGL, and MGI

MGG 21A, 21B, and 21P are BRL research wells.

\section{MGI WELL SERIES}

Description: Series I, Monitoring Grid Wells for Burial Grounds Bldg. Number: Old Burial Ground, 643-E

Map: Figures A-8, A-10, and A-12

\begin{tabular}{|c|c|c|c|c|c|c|c|c|c|}
\hline \multirow[b]{2}{*}{ Woll D } & \multicolumn{2}{|c|}{ 8R8 Coordinates } & \multicolumn{3}{|c|}{ Flevations (ft MBL) } & \multirow{2}{*}{$\begin{array}{l}\text { Eff. Well } \\
\text { Depth (ft) }\end{array}$} & \multirow[b]{2}{*}{ Casing } & \multirow[b]{2}{*}{ Installed } & \multirow[b]{2}{*}{ Abandong } \\
\hline & North & East & Ecreen Zone & Grnd. & Casing & & & & \\
\hline MGI 1 & 76381 & 54613.8 & $239.8-219.8$ & 278.1 & 279.8 & 60.0 & $4^{\prime \prime}$ Bteol & $03-01-76$ & \\
\hline MGI 3 & 75255 & 54768.6 & 239.6-219.6 & 280.3 & 281.6 & 62.0 & $4^{*}$ Btoel & $02-27-76$ & \\
\hline MGI 5 & 75131 & 64924.8 & $240.4-220.4$ & 276.5 & 278.4 & 58.0 & $4^{n}$ Steel & $02-26-76$ & \\
\hline MGI 7 & 75030 & 56099.1 & 242.1-222.1 & 276.1 & 278.1 & 56.0 & 4" Steel & $02-25-76$ & \\
\hline MGI 9 & 74908 & 55252.4 & 241.7-221.7 & 278.7 & 280.7 & 69.0 & $4^{n}$ Strol & $02-24-76$ & \\
\hline MGI 13 & 74660 & 56570.4 & 241.1-221.1 & 279.6 & 282.1 & 61.0 & $4^{n}$ PVC & $05-16-73$ & \\
\hline MGI 15 & 74538 & 55750.7 & $224.5-220.5$ & 277.0 & 279.5 & 69.0 & gn Steel & $05-08-73$ & \\
\hline MGI 17 & 74417 & 65890.3 & $243.5-223.5$ & 276.0 & 277.6 & 64.0 & $4^{n}$ PVC & $05.08-73$ & \\
\hline
\end{tabular}

Notes: The MGI Series is one of the Grid Series. The other Grid Beries are MGA, MGC, MGR, and MGG. 


\section{MHM SERIES}

Description: Geophysical Test Holes for the Horizontal Well Characterization Study in M Area

Bldg. Number: Not applicable

Map: Figure A-18

\begin{tabular}{|c|c|c|c|c|c|c|c|c|c|}
\hline \multirow[b]{2}{*}{ Well ID } & \multicolumn{2}{|c|}{ 8RS Coordinates } & \multicolumn{3}{|c|}{ Elevations (ft MSL) } & \multirow{2}{*}{$\begin{array}{l}\text { Eff. Well } \\
\text { Depth (ft) }\end{array}$} & \multirow[b]{2}{*}{ Casing } & \multirow[b]{2}{*}{ Installed } & \multirow[b]{2}{*}{ Abandoned } \\
\hline & North & East & Screen Zone & Grnd. & Caming & & & & \\
\hline MHM 1 & 102736.8 & 48878.8 & NA & 365.3 & 367.6 & NA & $* *$ & $04-26-90$ & \\
\hline MHM 2 & 102816.6 & 48922.0 & NA & 369.8 & 372.1 & NA & $* *$ & $05-03-90$ & \\
\hline MHM 3 & 102744.5 & 48890.0 & NA & 365.6 & 368.2 & NA & $\begin{array}{l}4^{n} \mathrm{PVC} / \\
4^{n} \mathrm{CS}\end{array}$ & 06.03 .90 & \\
\hline MHM 4 & 102778.0 & 48876.6 & NA & 368.3 & 370.3 & NA & 4" PVC & $05-11-90$ & \\
\hline MIIM 5 & 102811.3 & 48863.8 & NA & 366.2 & 368.5 & NA & $\begin{array}{l}4^{n} \text { PVC/ } \\
4^{n} \mathrm{CS}\end{array}$ & 06.11 .90 & \\
\hline
\end{tabular}

Notes: MAM 1 through 5 are not wells. They are geophyeical test holes with eolid casings and no screens. They cannot be nampled.

The casinge for MAM 3 and MAM 5 comprise alternating sections of 10-ft PVC and 2-ft C.8. according to the Daily Activity Reports for theee holes.

- MaTI 1 and 2 have 6-in. steel casinge inside 8-in. PVC casings. The well data for these wells indicate that the steel casinge extend as deeply as the PVC casinge.

\section{MHT WELL SERIES}

Description: Savannah River Laboratory (SRL) Monitoring Wells for the Horizontal Well Characterization Study in M Area

Bldg. Number: Not applicable

Map: Figure A-18

\begin{tabular}{|c|c|c|c|c|c|c|c|c|c|}
\hline & BR8 Coor & dinates & Dlevations if & MBL) & & Bff. Well & & & \\
\hline Well ID & North & East & Bcreen Zone & Grnd. & Casing & Depth (ft) & Casing & Insta'led & Abandoned \\
\hline MITI 10 & 102706.8 & 48765.6 & 209.7-204.7 & 362.7 & 364.9 & 160.2 & $4^{n}$ PVC & $08-07-90$ & \\
\hline MHT II & 102697.3 & 48760.2 & 238.0-217.9 & 362.5 & 364.4 & 146.5 & 4" PVC & $06-11-90$ & \\
\hline MHT 2C & 102747.1 & 48780.3 & 212.1-207.1 & 364.1 & 366.2 & 159.1 & 4" PVC & 04-06-90 & \\
\hline MHT 2I & 102756.6 & 48784.2 & $240.7-220.1$ & 364.5 & 966.5 & 146.4 & $4^{\prime \prime}$ PVC & $04-10.90$ & \\
\hline MHT 30 & 102704.3 & 48861.1 & 209.6-204.6 & 362.6 & 364.8 & 160.2 & 4" PVC & $04-23-90$ & \\
\hline MHY 9I & 102694.6 & 48856.8 & 237.1-217.1 & 362.1 & 364.3 & 147.2 & $4^{n}$ PVC & $04-18-90$ & \\
\hline MITT $4 C$ & 102778.9 & 48863.5 & 213.4-208.4 & 367.4 & 369.5 & 161.1 & 4" PVC & 04-12-90 & \\
\hline MITT 4I & 102772.1 & 48857.1 & $241.8-221.8$ & 366.8 & 368.9 & 147.1 & $4^{\prime \prime}$ PVC & $05.21-90$ & \\
\hline MITT $5 C$ & 102725.1 & 48905.9 & 210.1-205.1 & 364.1 & 366.2 & 161.1 & $4^{n}$ PVC & $06-29-90$ & \\
\hline MITT 6 I & 102721.7 & 48893.5 & $240.4-220.3$ & 363.9 & 366.0 & 145.7 & $4^{n}$ PVC & $05.29-90$ & \\
\hline MITT $6 C$ & 102810.8 & 48900.0 & 212.6-207.6 & 369.6 & 371.7 & 164.1 & $4^{*} \mathrm{PVC}$ & $04-26-90$ & \\
\hline MHT 6I & 102808.2 & 48891.0 & 244.4-224.4 & 369.4 & 371.3 & 146.9 & 4" PVC & $05-18-90$ & \\
\hline MHT 7C & 102788.9 & 48977.5 & 212.0-207.0 & 368.0 & 370.0 & 163.0 & 4" PVC & $06-21-90$ & \\
\hline MHT $7 \mathrm{I}$ & 102786.8 & 48967.3 & 239.9.219.9 & 367.9 & 370.0 & 160.1 & $4^{n}$ PVC & $06-26-90$ & \\
\hline MHT 80 & 102880.7 & 48970.2 & 212.3-207.3 & 369.3 & 371.5 & 164.2 & 4" PVC & $06-05-90$ & \\
\hline MHT 8D & 102875.8 & 48960.7 & $240.5-220.5$ & 369.5 & 371.7 & 151.2 & $4^{*}$ PVC & $06-05-90$ & \\
\hline MHT 90 & 102814.4 & 49016.6 & 214.7-209.7 & 367.7 & 369.6 & 169.9 & $4^{\prime \prime}$ PVC & $06-16-90$ & \\
\hline
\end{tabular}


MHT WELL SERIES (cont.)

\begin{tabular}{|c|c|c|c|c|c|c|c|c|c|}
\hline \multirow[b]{2}{*}{ Well ID } & \multicolumn{2}{|c|}{ ERS Coordinates } & \multicolumn{3}{|c|}{ Elevations (ft MSL) } & \multirow{2}{*}{$\begin{array}{l}\text { Eff. Well } \\
\text { Depth (ft) }\end{array}$} & \multirow[b]{2}{*}{ Casing } & \multirow[b]{2}{*}{ Installed } & \multirow[b]{2}{*}{ Abandone } \\
\hline & North & E.ast & Bcreen Zone & Grnd. & Casing & & & & \\
\hline IHT 9D & 102805.1 & 49018.1 & 242.6-222.6 & 367.6 & 369.8 & 147.2 & 4" PVC & $03-29.90$ & \\
\hline MTT $10 \mathrm{C}$ & 102892.3 & 49011.6 & 211.9.206.9 & 368.9 & 370.7 & 163.8 & 4" PVC & $06-19-90$ & \\
\hline MITT 10D & 102890.1 & 49001.2 & $240.0-219.9$ & 369.0 & 371.0 & 151.1 & $4^{n}$ PVC & $06-20-90$ & \\
\hline
\end{tabular}

Notes: According to Daily Activity Reports for MITr sc, the original borebole was not straight and was abandoned. This borehole was grouted up 3-26-90 and toppod off 6-27-90.

\section{MHV WELL SERIES}

Description: Vapor Probes Adjacent to the Horizontal Wells in M Area Bldg. Number: Not applicable

Map: Figure A-18

\begin{tabular}{|c|c|c|c|c|c|c|c|c|c|}
\hline \multirow[b]{2}{*}{ Well ID } & \multicolumn{2}{|c|}{ SRs Coordinates } & \multicolumn{3}{|c|}{ Elevations (A MSL) } & \multirow{2}{*}{$\begin{array}{l}\text { Eff. Well } \\
\text { Depth (ft) }\end{array}$} & \multirow[b]{2}{*}{ Caging } & \multirow[b]{2}{*}{ Installed } & \multirow[b]{2}{*}{ Abandoned } \\
\hline & North & East & Bcreen Zone & Grnd. & Caning & & & & \\
\hline MHV IA & 102749.3 & 48842.0 & 270.6-265.6 & 365.6 & 387.6 & 102.0 & 1* PVC & $05-09-90$ & \\
\hline MHV $1 B$ & 102749.3 & 48842.0 & 295.6-290.6 & 385.6 & 367.6 & 77.0 & 1. PVC & $05-10-90$ & \\
\hline MAV 1C & 102749.3 & 48842.0 & $321.6-316.6$ & 386.6 & 367.6 & 62.0 & 1* PVC & $05-11.90$ & \\
\hline MHV 2A & 102756.9 & 48903.2 & $271.4-266.4$ & 366.4 & 388.2 & 101.8 & 1" PVC & $05-29-90$ & \\
\hline MHV $2 B$ & 102765.9 & 48903.2 & 296.4291 .4 & 396.4 & 388.2 & 76.8 & 1" PVC & $05-30-90$ & \\
\hline MrV 2C & 102766.9 & 48903.2 & $322.4-317.4$ & 386.4 & 868.2 & 60.8 & 1* PVC & $08-31.90$ & \\
\hline MHV 3A & 102774.7 & 48874.1 & 273.2-268.2 & 368.2 & 370.3 & 102.1 & 1- PVC & $05-14-80$ & \\
\hline MHV 3B & 102774.7 & 48874.1 & 298.8-293.8 & 368.2 & 370.3 & 76.6 & 1* PVC & $06-16-90$ & \\
\hline MHV 3C & 102774.7 & 48874.1 & $323.2-318.2$ & 368.2 & 370.3 & 52.1 & 1* PVC & $05-16-90$ & \\
\hline MITV $4 A$ & 102841.7 & 48842.5 & 272.1-287.1 & 366.1 & 368.6 & 101.6 & 1* PVC & $06-13-90$ & \\
\hline MHV $4 B$ & 102841.7 & 48842.5 & 296.1-291.1 & 366.1 & 368.5 & 77.4 & 1" PVC & $06-16.90$ & \\
\hline MHV 4C & 102841.7 & 48842.6 & $323.6-318.6$ & 366.1 & 368.7 & 60.1 & 1" PVC & $08-18-90$ & \\
\hline MHV 6A & 102878.8 & 49917.6 & 284.2-279.2 & 399.2 & 371.6 & 92.4 & 1" PVC & $08-08-90$ & \\
\hline MHV 6B & 102878.8 & 48917.5 & $301.2-296.2$ & 369.2 & 371.6 & 75.3 & 1" PVC & $06-07.90$ & \\
\hline MHV $6 \mathrm{C}$ & 102878.8 & 48917.6 & $324.2-319.2$ & 399.2 & 371.6 & 62.4 & 1* PVC & $06-08-90$ & \\
\hline
\end{tabular}

Noten: A clucter in the MEV Beries is composed of three wells with ceparate caeinge and ecreens placed side by side in the same location. Coneequently, all welle in a cluster have nearly identical coondinates, top of canings, and ground elevations.

MPT 1 Well (see RWM WELL SERIES) 
MSB WELL SERIES

Description: M-Area Hazardous Waste Management Facility (HWMF) and Plume Definition Wells

Bldg. Number: HWMF: 904-51G, -112G (Lost Lake)

Plume Definition Wells: Not applicable

Map: Figures A-17, A-18, and A-19

\begin{tabular}{|c|c|c|}
\hline \multirow[b]{2}{*}{ Well ID } & \multicolumn{2}{|c|}{ SRS Coordinates } \\
\hline & North & $\underline{\text { Eant }}$ \\
\hline MSB 1 & 101824.2 & 48486.1 \\
\hline $\operatorname{MSB} 1 A^{1}$ & 101833.7 & 48467.3 \\
\hline MSB 1B $^{1}$ & 101833.0 & 48483.2 \\
\hline MSB $1 C^{1}$ & 101832.5 & 48512.7 \\
\hline $\mathrm{MBB} 1 \mathrm{CC}^{1}$ & 101892.5 & 48498.0 \\
\hline M8B 1D' & 101833.4 & 48462.2 \\
\hline M\&B 2 & 101999.2 & 48741.9 \\
\hline MSB $2 A^{2}$ & 102028.3 & 48746.4 \\
\hline MSB 2B' & 101997.9 & 48748.2 \\
\hline MSB 2C & 101982.5 & $\$ 8749.3$ \\
\hline MSB 2D' & 102014.0 & 48755.7 \\
\hline MSB 3 & 102181.6 & 48530.7 \\
\hline MSB $3 A^{\prime}$ & 102189.9 & 48553.7 \\
\hline MSB $3 B^{1}$ & 102191.7 & 48668.0 \\
\hline MSB $3 C^{1}$ & 102189.6 & 48538.5 \\
\hline MSB 3D' & 102188.6 & 48524.6 \\
\hline MSB 4 & 102010.4 & 48313.8 \\
\hline $\operatorname{MSB} 4 A^{1}$ & 101993.4 & 48319.0 \\
\hline MSB $4 B^{1}$ & 101978.3 & 48912.8 \\
\hline MSB $4 C^{2}$ & 101963.2 & 48313.6 \\
\hline MSB 4D' & 102007.6 & 48311.7 \\
\hline MBB 6 & $10176 R .7$ & 46983.8 \\
\hline MSB $6 A^{1}$ & 101971.6 & 46998.7 \\
\hline MSB 5B $^{1}$ & 101971.1 & 46983.6 \\
\hline $\mathrm{MBB}$ 6C $\mathrm{C}^{1}$ & 101970.4 & 46968.6 \\
\hline MSB 6 & 101080.1 & 46462.6 \\
\hline MBB $6 A^{2}$ & 101133.8 & 46319.9 \\
\hline MSB $6 B^{\prime}$ & 101148.5 & 46321.6 \\
\hline MSB $6 C^{1}$ & 101169.1 & 46324.1 \\
\hline MSB 7 & 100730.7 & 46786.9 \\
\hline MSB $\mathbf{7 A}^{2}$ & 100585.7 & 46726.1 \\
\hline MBB $7 B^{1}$ & 100597.6 & 46718.1 \\
\hline MSB $7 C^{1}$ & 100609.2 & 46709.1 \\
\hline MSB 8 & 100944.0 & 47145.4 \\
\hline M8B $8 A^{1}$ & 100815.1 & 47293.2 \\
\hline MSB 8B $^{1}$ & 100805.8 & 47281.9 \\
\hline MSB $8 C^{1}$ & 100793.2 & 47264.6 \\
\hline MSB $9 A^{8}$ & 102236.7 & 48242.5 \\
\hline MSB 9B' & 102239.4 & 48251.7 \\
\hline MSB $9 C^{d}$ & 102245.6 & 48273.0 \\
\hline MSB $10 A^{\circ}$ & 102451.8 & 47954.4 \\
\hline MSB 10B & 102488.2 & 47943.1 \\
\hline MSB $10 C^{8}$ & 102465.6 & 47951.1 \\
\hline MSB 10D & 102476.9 & 47947.4 \\
\hline MSB 11A & 102638.9 & 48577.6 \\
\hline $\mathrm{MSB} 11 \mathrm{~B}^{\mathrm{s}}$ & 102648.9 & 48578.6 \\
\hline
\end{tabular}

Elevations (ft MSL)

Screen Zone Grnd. Casing

239.8-219.8

253.4-223.4

142.7.138.0

166.1-161.4

192.6-187.9

229.8-210.4

263.0-243.0

252.7.222.7

150.3-145.6

194.7-190.0

230.1.210.7

255.0-235.0

259.8-229.8

145.8.141.1

193.7.189.0

280.7-211.2

256.7-236.7

254.9.224.9

149.1-138.4

168.1-163.3

228.4-209.0

247.8-227.8

247.6-217.6

136.6-131.8

188.6-183.8

248.4-228.4

241.9-211.9

$130.3-125.6$

194.5-189.8

250.2-230.2

242.1-212.1

147.6-14R.8

200.2-195.6

248.2-228.2

242.6-212.5

160.9-146.2

195.9.191.2

143.7.138.7

208.7-203.7

241.3-221.3

122.9-117.9

154.5.149.6

209.7-205.5

249.7-229.7

135.4-130.4

165.6-160.6
Eff. Well

\begin{tabular}{|c|c|c|c|}
\hline Depth (tt) & Casing & Installed & Abandoned \\
\hline 133.6 & $4^{n}$ Steel & $11-12-79$ & $01-01-89^{4}$ \\
\hline 130.0 & $4^{n}$ PVC & $01-01-83^{*}$ & \\
\hline 216.8 & $4^{n}$ PVC & $10-18-90$ & \\
\hline 4.7 & $4^{n}$ PVC & $08-07-90$ & \\
\hline 167.0 & 4" PVC & $10-26-90$ & \\
\hline 144.5 & $4^{\prime \prime} \mathrm{PVC}$ & $10-10-90$ & \\
\hline 109.3 & $4^{n}$ Steel & $11-19-79$ & $01.01-83^{*}$ \\
\hline 130.0 & 4" PVC & $01.01-83 *$ & \\
\hline 209.0 & $4^{\prime \prime}$ PVC & $08-30-90$ & \\
\hline 164.7 & 4" PVC & $08-30-90$ & \\
\hline 143.1 & 4" PVC & $11.16-90$ & \\
\hline 124.6 & $4^{n}$ Btoel & $12-04-79$ & $01-01-83^{*}$ \\
\hline 130.0 & 4" PVC & $01-01-83^{*}$ & \\
\hline 219.9 & $4^{\prime \prime}$ PVC & 09.17 .90 & \\
\hline 171.8 & $4^{\prime \prime}$ PVC & 09.20 .90 & \\
\hline 149.3 & $4^{n}$ PVC & 09.20 .90 & \\
\hline 118.4 & $4^{*}$ Steel & $03-21-80$ & $01-01-83^{*}$ \\
\hline 130.0 & $4^{\prime \prime}$ PVC & 10-08-82 & \\
\hline 216.9 & $4^{*}$ PVC & $1008-90$ & \\
\hline 191.9 & $4^{n}$ PVC & $08-23-90$ & \\
\hline 146.6 & 4" PVC & $09-28.90$ & \\
\hline 111.3 & $4^{*}$ Steel & $02-10-81$ & $10-01-82^{*}$ \\
\hline 127.0 & $4^{n}$ PVC & $10-25-82$ & \\
\hline 213.7 & $4^{n}$ PVC & $05-25-90$ & \\
\hline 161.9 & $4^{n}$ PVC & $05-25-90$ & \\
\hline 111.3 & $4^{n}$ Steel & $02-03-81$ & $10-01-82 *$ \\
\hline 132.0 & 4" PVC & $10-01-82 *$ & \\
\hline 219.7 & 4" PVC & 05.09 .90 & \\
\hline 164.4 & $4^{\prime \prime}$ PVC & $05-10-90$ & \\
\hline 110.6 & $4^{\prime \prime}$ Stoel & $02-27-81$ & $10.01 .82^{*}$ \\
\hline 132.4 & $4^{n}$ PVC & $10.01 .82 *$ & \\
\hline 201.4 & $4^{n}$ PVC & $04-18-90$ & \\
\hline 149.1 & 4" PVC & $04-1890$ & \\
\hline 111.2 & $4^{\prime \prime}$ Bteel & $02-24-81$ & $10-01-82^{*}$ \\
\hline
\end{tabular}

131.7 4" PVC 10-01-82*

197.7 4" PVC $04-05.90$

$152.8 \quad 4^{n}$ PVC 04-18-90

220.7 PVC 07.28-81

165.9 PVC

$137.8 \quad 4^{n}$ PVC

$237.1 \quad 4^{\prime \prime}$ PVC

$205.2 \quad 4^{n}$ PVC

$150.5 \quad 4^{\prime \prime}$ PVC

$125.8 \quad 4^{\prime \prime}$ PVC

$234.6 \quad 4^{\prime \prime}$ PVC

4" PVC
12-18-81

09.03.81

09.11 .81

09.01 .81

09.09.81

10.14-81

10.09-81

(series continued) 
MSB WELL SERIES (cont.)

\begin{tabular}{|c|c|c|}
\hline & BRS Coor & dinatos \\
\hline Well ID & North & East \\
\hline MBB $11 C^{\prime}$ & 102658.6 & 48579.4 \\
\hline MSB 11D" & 102669.6 & 48679.7 \\
\hline MBB 11E' & 102678.6 & 48679.6 \\
\hline MSB 11P* & 102629.3 & 48577.0 \\
\hline MBB 12A' & 102283.2 & 47138.2 \\
\hline MBB $12 B^{\circ}$ & 102251.8 & 47139.6 \\
\hline MBB $12 C^{\circ}$ & 102274.4 & 47138.4 \\
\hline MSB 12D' & 102262.2 & 47139.7 \\
\hline MSB 12TA' & 102266.7 & 47127.3 \\
\hline MBB 12TB' & 102260.1 & 47139.0 \\
\hline MSB 13A' & 101725.7 & 47525.4 \\
\hline MSB 13B' & 101735.7 & 47523.5 \\
\hline MBB 13C & 101745.7 & 47521.9 \\
\hline MBB $13 C^{1}$ & 101728.8 & 47525.7 \\
\hline MSB 13D' & 101778.1 & 47517.6 \\
\hline MBB $14 A^{\circ}$ & 101629.6 & 48521.9 \\
\hline MSB 148' & 101639.0 & 48519.1 \\
\hline MSB 14C' & 101648.6 & 48517.3 \\
\hline MBB 16A* & 102989.6 & 48827.0 \\
\hline MBB 15AA & 102963.2 & 48818.5 \\
\hline MBB 15C & 103002.1 & 48834.0 \\
\hline MEB 15D & 102971.2 & 48827.6 \\
\hline MBB 16A & 103693.9 & 48966.1 \\
\hline MSB 16C & 109714.1 & 48970.5 \\
\hline MBB 17A' & 101976.6 & 46246.7 \\
\hline $\mathrm{MSB} 17 \mathrm{~B}^{4}$ & 101994.6 & 46237.7 \\
\hline MBB $17 B B$ & 102009.6 & 46220.8 \\
\hline MBB 17C & 102004.6 & 46234.3 \\
\hline MBB 17D & 102056.9 & 46226.2 \\
\hline MBB 18A" & 100416.1 & 46110.4 \\
\hline MBB 18B' & 100424.1 & 46116.7 \\
\hline MSB $18 C^{4}$ & 100450.9 & 46121.4 \\
\hline MSB 19A & 100989.0 & 60934.4 \\
\hline MSB 19B & 100999.3 & 60934.8 \\
\hline MBB 19C' & 100992.1 & 50942.4 \\
\hline MBB 20A & 103645.1 & 46060.5 \\
\hline MBB 20C & 103556.3 & 46088.8 \\
\hline M8B 21A' & 103987.0 & 47217.2 \\
\hline MAB 21B & 104000.1 & 47271.8 \\
\hline MSB 21C & 103973.0 & 47234.6 \\
\hline MSB 21TA' & 103980.9 & 47218.2 \\
\hline MBB 221 & 102186.5 & 48608.8 \\
\hline M8B 23 & 104312.0 & 49294.0 \\
\hline M8B 23A & 104314.9 & 49290.7 \\
\hline MBB 23B & 104356.6 & 49286.4 \\
\hline M8B 2STA & 104298.8 & 64225.8 \\
\hline MBB 24 & 104614.4 & 49842.9 \\
\hline MBB 24A & 104825.3 & 49845.3 \\
\hline MBB 26 & 103498.8 & 49688.9 \\
\hline MBB 25A & 103504.8 & 49657.9 \\
\hline MBB 26 & 104612.8 & 48941.7 \\
\hline MSB 26A & 104602.3 & 48440.7 \\
\hline MBB 26B & 104646.7 & 48944.6 \\
\hline MBB 27 & 104972.8 & 49487.7 \\
\hline
\end{tabular}

Blevations (ft MSL)

Bcreen Zone Grnd. Caning

182.4-177.4

208.3-203.3

250.9-230.9

242.5-222.5

120.9-116.9

161.2-166.2

182.9.177.9

244.3-224.3

(109.7-119.7)

13.7. (6.3)

134.9.129.9

176.2-171.2

242.6-222.6

196.8-192.0

231.6-211.6

164.6-144.6

193.7.188.7

249.7-223.7

167.2-162.2

147.4-142.7

$260.4-240.4$

241.7-222.2

166.2-161.2

$244.0-224.0$

169.6-184.6

190.0.185.0

137.6-132.9

253.9-393.3

233.1-213.6

162.3-157.3

197.0-192.0

227.3-207.3

119.6-114.6

147.4-142.4

218.1-198.1

161.2-166.2

232.4-212.4

167.8-162.8

147.5-142.8

231.8-211.8

24.0. 18.7

241.7.221.7

250.4-230.4

$62.4-52.4$

176.1-171.1

65.460 .4

243.9-223.9

178.8.168.8

244.7-224.7

159.7-169.7

240.5-220.5

179.2-169.2

137.2-132.4

244.0-234.0

$\mathbf{3 6 3 . 0} 364.9$

$\mathbf{3 6 3 . 3 \quad 3 3 6 . 2}$

$363.4 \quad 365.2$

$\mathbf{3 4 6 . 9} \mathbf{3 4 7 . 8}$

$346.5 \quad 348.4$

$346.0 \quad 347.9$

$346.3 \quad 348.1$

$\mathbf{3 4 6 . 3} \mathbf{3 4 8 . 6}$

$346.7 \quad 348.9$

$343.3 \quad 345.2$

$343.7 \quad 345.6$

$343.6 \quad 345.7$

$\mathbf{3 4 4 . 8} \mathbf{3 4 6 . 9}$

346.5 347.7

$\mathbf{3 4 6 . 5} \mathbf{3 4 8 . 3}$

\begin{tabular}{l}
$346.7 \quad \mathbf{3 4 8 . 7}$ \\
\hline
\end{tabular}

$\mathbf{3 4 7 . 0} \mathbf{3 4 8 . 7}$

$\mathbf{3 6 5 . 2} \quad \mathbf{3 6 7 . 2}$

$\mathbf{3 6 7 . 4} \mathbf{3 6 9 . 6}$

$364.6 \quad 366.6$

$\begin{array}{lll}366.7 & 368.8\end{array}$

$364.9 \quad 366.7$

$\mathbf{3 6 5 . 0} \quad \mathbf{3 6 6 . 6}$

$366.3 \quad 358.0$

$\begin{array}{ll}356.4 & 357.9\end{array}$

$\begin{array}{lll}\mathbf{3 5 7 . 6} & \mathbf{3 5 9 . 9}\end{array}$

$366.3 \quad 958.1$

$358.1 \quad 360.2$

$358.3 \quad \mathbf{3 4 0 . 2}$

$\mathbf{3 3 8 . 5} \quad \mathbf{3 4 0 . 3}$

$338.6 \quad 340.6$

$298.1 \quad 299.5$

$298.2 \quad 299.9$

$298.1 \quad 300.2$

$361.9 \quad 364.0$

$361.2 \quad 363.3$

$351.2 \quad 353.4$

$363.6 \quad 366.3$

$361.6 \quad 353.4$

$352.7 \quad 354.7$

$366.7 \quad 359.0$

$\mathbf{3 7 0 . 4} 371.8$

$370.5 \quad 371.6$

$370.1 \quad 371.6$

$\begin{array}{ll}370.4 & 372.9\end{array}$

$378.9 \quad 380.2$

$379.9 \quad 381.6$

$\begin{array}{ll}\mathbf{3 6 4 . 7} & \mathbf{3 6 6 . 9}\end{array}$

$\mathbf{3 6 4 . 7} \mathbf{3 6 6 . 4}$

$369.5 \quad 361.6$

$369.2 \quad 360.9$

$361.0 \quad 363.2$

$374.0 \quad 375.6$
Eff. Well

Depth (t)

$363.2 \quad 364.8$

187.

161.9

134.3

142.3

231.9

192.2

170.0

123.8

462.2

365.2

215.3

174.4

123.1

164.9

136.2

203.8

160.0

125.0

206.0

236.8

126.2

146.6

206.5

142.6

203.4

172.9

226.4

124.8

146.6

182.9

148.3

139.3

184.9

167.6

102.1

197.8

140.9

200.6

212.6

141.6

336.0

137.3

141.4

319.1

200.6

312.6

166.3

212.8

142.2

206.7

141.1

191.7

230.8

141.6

\begin{tabular}{|c|c|}
\hline ng & 1stalled \\
\hline & $10-13-81$ \\
\hline & $10.15-81$ \\
\hline & $10-16-81$ \\
\hline & $12-01-81$ \\
\hline & $10-29-81$ \\
\hline & $10-14-81$ \\
\hline & $10-19-81$ \\
\hline & $10-05-81$ \\
\hline (" Stoel & 12.01 .81 \\
\hline 1" Steol & $12.01-81$ \\
\hline & $10-22-81$ \\
\hline & $10-28-81$ \\
\hline & $10-07-81$ \\
\hline & $11-13-90$ \\
\hline A m & $08-23-90$ \\
\hline & $10-19-81$ \\
\hline & $10-20-81$ \\
\hline & $10-09-81$ \\
\hline 8.75" PVC & $11-09-81$ \\
\hline & $02-20-89$ \\
\hline 3.75' PVC & $11-10-81$ \\
\hline & $09-06-88$ \\
\hline 3.75" PVC & $11-16-81$ \\
\hline 3.75" PVC & $12-09-81$ \\
\hline 3.75" PVC & $11-02-81$ \\
\hline 3.76" PVC & 12-16-81 \\
\hline 4" $1 v C$ & $01-09-88$ \\
\hline $3.75^{\circ}$ & $11-03-81$ \\
\hline & $09-16-88$ \\
\hline 3.75" P & $12-09-81$ \\
\hline 3.75" & $12-01-81$ \\
\hline PVC & $12-01-81$ \\
\hline 3.75" & $12-08-81$ \\
\hline s.75" & $12-11-81$ \\
\hline $3.75^{n}$ & $19-09-81$ \\
\hline 3.76" & $11-20-81$ \\
\hline 3.75" & $11.28-31$ \\
\hline $3.75^{\prime \prime}$ & $11-19-81$ \\
\hline 4" PN & $02-20-89$ \\
\hline 3.76" PVC & $11.23-81$ \\
\hline $4^{\circ}$ PVC & $12-01-81$ \\
\hline 4" PVC & $06-01-82$ \\
\hline 4" PVC & $08-01-82$ \\
\hline 4" PVC & $03-22-83$ \\
\hline 4" PVC & 02-16-83 \\
\hline $\begin{array}{l}4^{\prime \prime} \text { 8te } \\
\text { PvC }\end{array}$ & $09-22-82$ \\
\hline PVC & 02.07 .83 \\
\hline PVC & $08-01-82$ \\
\hline 4" PVC & 02.02 .83 \\
\hline PVC & $08-01-82$ \\
\hline & $01-12-83$ \\
\hline & $02-13-89$ \\
\hline & $08-01.82$ \\
\hline
\end{tabular}

PVC

08-01-82
Abandoned 
MSB WELL SERIES (cont.)

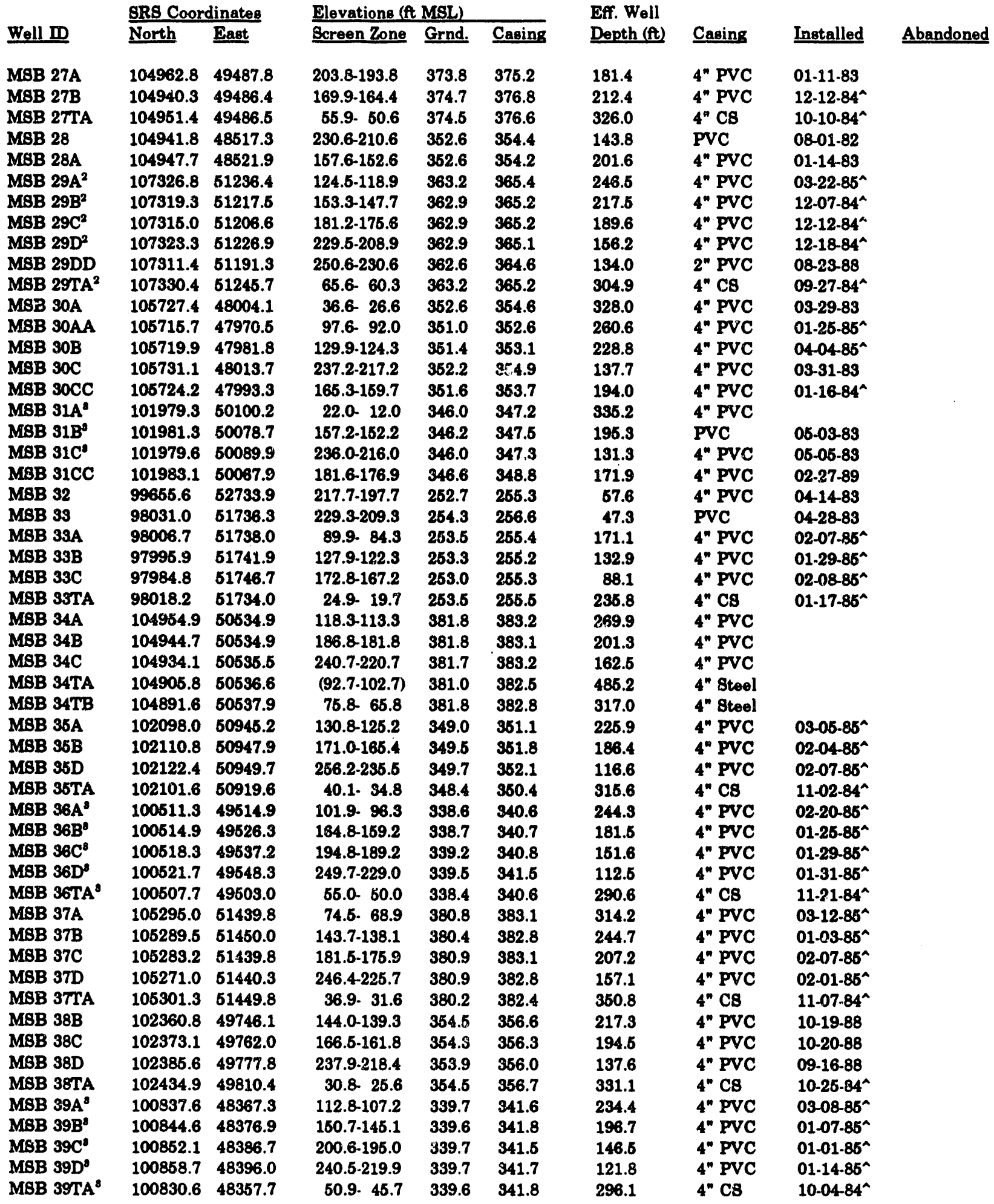

(series continued) 
MSB WELL SERIES (cont.)

\begin{tabular}{|c|c|c|}
\hline \multirow[b]{2}{*}{ Well ID } & \multicolumn{2}{|c|}{ BRs Coordinates } \\
\hline & North & Past \\
\hline MSB $40 A^{\circ}$ & 97672.8 & 48279.4 \\
\hline MSB 40B' & 97685.0 & 48281.6 \\
\hline MBB 40C' & 97697.8 & 48283.6 \\
\hline MSB 40D' & 97709.3 & 48285.1 \\
\hline M8B 4OTA' & 97660.4 & 48277.2 \\
\hline MSB 41A & 102184.4 & 53424.1 \\
\hline MBB $41 B$ & 102194.5 & 53417.8 \\
\hline MSB $41 \mathrm{C}$ & 102209.9 & 63410.6 \\
\hline MBB 41D & 102219.4 & 58403.7 \\
\hline MBB 41TA & 102176.6 & 63429.7 \\
\hline MBB 42A & 104657.9 & 61582.3 \\
\hline MBB 42B & 104569.8 & 61582.8 \\
\hline MSB $42 \mathrm{C}$ & 104681.9 & 61682.8 \\
\hline MSB 42D & 104585.2 & 61582.5 \\
\hline MBB 42TA & 104646.6 & 61581.7 \\
\hline MSB 43A 2 & 107276.3 & 49293.7 \\
\hline MSB 43B & 107274.6 & 49811.8 \\
\hline$\therefore$ :48B 49D2 & 107274.2 & 49322.0 \\
\hline MSB 49DD & 107273.0 & 49341.2 \\
\hline MBB 45TA ${ }^{2}$ & 107275.8 & 49281.8 \\
\hline MSB 4AA & 109298.5 & 51108.9 \\
\hline MBB 4AB & 103298.2 & 51098.4 \\
\hline MSB 4 C & 103296.3 & 61106.6 \\
\hline MBB $45 A$ & 109998.1 & 60554.7 \\
\hline MAB $45 B$ & 109987.9 & 60556.3 \\
\hline $\mathrm{MBB} 45 \mathrm{C}$ & 105997.7 & 60554.7 \\
\hline MBB 48A & 109098.6 & 60548.3 \\
\hline MBB $48 B$ & 103102.4 & 60567.5 \\
\hline MBB 46C & 103098.6 & 60548.7 \\
\hline $\mathrm{MBB} 4 \mathrm{~TB}$ & 106978.6 & 62207.2 \\
\hline MBB $47 B B$ & 100999.7 & 52234.4 \\
\hline MBB 47C & 106969.2 & 62195.6 \\
\hline MBB $47 \mathrm{D}$ & 106960.1 & 62184.0 \\
\hline MBB $47 T A$ & 106987.7 & 62219.0 \\
\hline MBB 48A & 107986.6 & 54099.8 \\
\hline MBB A8B & 107945.0 & 64112.2 \\
\hline M8B $48 C$ & 107917.5 & 54077.0 \\
\hline MSB 48D & 107914.4 & 64056.3 \\
\hline MABB 48TA & 107925.8 & 64089.2 \\
\hline MBB 49A & 99769.0 & 46884.6 \\
\hline MBB 49B & 99797.8 & 46888.2 \\
\hline MgB 49D & 99724.9 & 45868.4 \\
\hline MBB БOB & 96433.0 & 51003.6 \\
\hline MBB EOD & 96416.7 & 51044.1 \\
\hline M8B Б1B & 96992.7 & 62818.0 \\
\hline MBB 61D & 97016.7 & 52816.2 \\
\hline MBB 61DD & 97006.2 & 62830.3 \\
\hline MSB 62B & 103077.7 & 53418.4 \\
\hline M8B E2D & 108062.7 & 53416.8 \\
\hline MEB 63B & 108443.6 & 64574.3 \\
\hline MBB $53 \mathrm{C}$ & 106456.2 & 54540.6 \\
\hline MBB E3D & 108448.2 & 64553.1 \\
\hline MBB SAB & 108446.8 & 52970.5 \\
\hline MSB B4C & 108447.4 & 52955.7 \\
\hline
\end{tabular}

Blevations (A MBL)

ScreenZone Grad. Caning

117.7-112.1

155.8-150.2

192.8-187.2

237.0-216.4

30.7- 25.6

89.1- 83.5

115.0-109.4

$162.8-147.2$

247.7-227.0

28.1- 22.9

130.8-125.2

167.8-162.2

205.8-200.2

248.7-228.2

47.4 42.1

141.8-136.2

176.7-171.1

221.6-201.0

243.4-223.3

41.9. 36.7

134.1-124.1

184.1-174.1

299.9-229.9

138.9.128.9

189.8179 .8

243.4-233.1

129.7-119.7

189.7.179.7

246.8-296.8

171.1-165.5

120.6-115.9

202.4-196.8

246.1-226.5

65.2. 60.2

129.9-125.2

$168.8-164.1$

180.8-176.0

243.4221.9

108.4-103.1

77.4 72.7

116.2-110.6

236.0-216.3

164.8-149.2

210.9-190.8

159.9-154.3

218.8-199.1

235.8-216.2

171.3-165.7

251.4-231.7

152.6-147.9

192.9-188.1

244.6-223.3

136.6.130.9

175.9-170.3

$319.2 \quad 321.2$

$319.7 \quad 321.7$

$320.5 \quad 322.1$

$320.9 \quad 322.9$

$319.0 \quad 320.8$

$321.9 \quad 323.8$

$322.2 \quad 324.0$

$322.5 \quad 324.6$

$322.8 \quad 325.0$

$321.7 \quad 328.7$

$374.6 \quad 376.6$

$374.4 \quad 376.6$

374.4376 .5

$374.3 \quad 376.6$

\begin{tabular}{ll|l}
374.6 & 376.7
\end{tabular}

$356.6 \quad 367.9$

$355.7 \quad 358.0$

$366.0 \quad 357.6$

$356.4 \quad 358.1$

$356.4 \quad 357.6$

$\mathbf{3 7 3 . 9} \quad \mathbf{3 7 6 . 9}$

$374.1 \quad 377.1$

$374.9 \quad 377.9$

$378.1 \quad 381.1$

$378.1 \quad 381.1$

$378.1 \quad 381.1$

$\begin{array}{ll}369.7 & \mathbf{5 7 2 . 7}\end{array}$

$\begin{array}{lll}\mathbf{5 7 0 . 7} & \mathbf{5 7 3 . 7}\end{array}$

\begin{tabular}{l|l}
$\mathbf{3} 89.8$ & $\mathbf{3 7 2 . 8}$
\end{tabular}

$\mathbf{3 6 6 . 3} \mathbf{3 8 9 . 0}$

$366.6 \quad 369.1$

$\$ 66.7 \quad 399.3$

$366.6 \quad 369.2$

$366.3 \quad 369.0$

$359.9 \quad 362.2$

$359.8 \quad 361.9$

$\mathbf{3 6 0 . 8} \quad 362.9$

$360.4 \quad 563.2$

$360.4 \quad 362.4$

$333.4 \quad 335.4$

$331.6 \quad 334.8$

$331.5 \quad 334.2$

$221.0 \quad 224.0$

$220.5 \quad 223.6$

$280.8 \quad 263.6$

$259.8 \quad 262.5$

$261.0 \quad 263.3$

$319.2 \quad 321.9$

$319.2 \quad 321.8$

$342.6 \quad 344.6$

$342.9 \quad 346.6$

$342.6 \quad 346.1$

$371.0 \quad 373.7$
Eff. Well

Depth (A) Casing Installed Abandoned

$\mathbf{3 7 0 . 9} \quad \mathbf{3 7 3 . 7}$
$209.1 \quad 4 "$ PVC 03-01.85^

$171.5 \quad 4 "$ PVC 01-31-85

134.9 4" PVC 02-04-85^

106.6 4" PVC 02-07-86^

$296.5 \quad 4^{\circ} \mathrm{CS} \quad 11-30-84^{\wedge}$

240.3 4" PVC 12-13-84"

214.6 4" PVC 02-25-85^

177.4 4" PVC 02-11-85^

98.0 4" PVC 02.19-86"

$300.8 \quad 4^{\prime \prime} \mathrm{CS} \quad 01-11-85^{\wedge}$

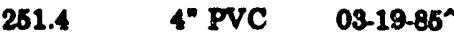

$176.3 \quad 4^{\prime \prime}$ PVC 01.24-86"

148.3 4" PVC 01-30-85"

$394.6 \quad 4^{\circ} \mathrm{CS} \quad 10.31-84^{\circ}$

221.7 4" PVC 0401-86

186.9 4" PVC 12.12-84"

156.5 4" PVC 01.03-86

$134.8 \quad 2^{*}$ PVC 08-23-88

$320.9 \quad 4^{\prime \prime} \mathrm{CB} \quad 02-25-84^{\wedge}$

252.8 4" PVC

203.0 4" PVC

$148.0 \quad 2^{n}$ PVC

252.2 4" PVC

201.3 4" PVC

$148.0 \quad$ 2" PVC

255.0 4" PVC

194.0 4" PVC

136.0 2" PVC

203.5 4" PVC

253.2 4" PVC

172.5 4" PVC

142.7 4" PVC

318.8

237.0

207.8

186.9

141.3

259.3

262.7

224.2

117.9

74.8

32.7

109.2

63.4

47.1

166.2

90.1

196.7

157.4

121.8

242.8

203.4
4. CS

$4 "$ PVC

4 PVC

4" PVC

4" PVC

**

$44^{\circ}$ PVC

4" PVC

4" PVC

4" PVC

4" PVC

4" PVC

4" PVC

4" PVC

4" PVC

4" PVC

4" PVC

4" PVC

4" PVC

PVC

4" PVC
07-10.87

$10.16-89$

07-14-87

08-07-87

01-12-90

01-16-90

05-25-89

01-16-90

01-20-89

07-31-87

08-10-87

05-20-87

08-04-87

06-26-87

08-31-87

08-04-87

07-21-87

08-06-87

02.07 .89

03.12 .87

07-24-87

$07.28-87$
06-22-87 $214.344^{\prime \prime}$ PVC 01-16-86 
MSB WELL SERIES (cont.)

\begin{tabular}{|c|c|c|c|c|c|c|c|c|c|}
\hline & SRS Coor & dinates & Elevations if & MSL) & & Eff. Well & & & \\
\hline Well ID & North & East & Screen Zone & Grnd. & Casing & Depth (ft) & Casing & Installed & Abandoned \\
\hline MSB 64D & 108461.5 & 52984.5 & 244.5-223.5 & 371.1 & 374.0 & 150.5 & 4" PVC & & \\
\hline MSB 54TA & 108446.3 & 52985.8 & 81.0. 75.4 & 371.0 & 373.8 & 298.4 & Cs & 07-22-87 & \\
\hline MBB 65B & 108342.4 & 52006.2 & $152.9 \cdot 148.2$ & 366.9 & 368.9 & 220.7 & $4^{\prime \prime}$ PVC & $01-03-90$ & \\
\hline MSB 65C & 108324.6 & 52029.7 & $189.4-184.7$ & 367.4 & 369.5 & 184.8 & $4^{n}$ PVC & $04-28-89$ & \\
\hline MSB 65D & 108391.4 & 52032.5 & 246.5-224.3 & 365.5 & 368.4 & 144.1 & 4" PVC & & \\
\hline MSB 65HC & 108338.7 & 52020.1 & 218.9-214.2 & 366.9 & 368.8 & 154.6 & $4^{n}$ PVC & $10-06-90$ & \\
\hline MSB 65TA & 108322.8 & 52014.7 & 91.7. 86.3 & 366.7 & 368.8 & 282.5 & $* *$ & 12-06-89 & \\
\hline MSB 66D & 108463.6 & 44207.9 & 232.2-210.9 & 277.2 & 279.8 & 68.9 & $4^{n}$ PVC & & \\
\hline MSB 67D & 101829.5 & 48701.5 & 229.6-210.1 & 364.1 & 356.2 & 146.1 & $4^{n}$ PVC & $10-26-90$ & \\
\hline MSB 58D & 102200.6 & 48693.5 & 230.6-211.2 & 355.9 & 357.9 & 146.7 & $4^{n} \mathrm{PVC}$ & $10-26-90$ & \\
\hline MBB 69D & 102182.2 & 48314.8 & 229.3-209.9 & 367.3 & 369.3 & 149.4 & $4^{n}$ PVC & $09-26-90$ & \\
\hline MSB 60D & 101895.6 & 48326.8 & 228.3-208.9 & 352.3 & 354.6 & 146.6 & $4^{\prime \prime}$ PVC & $10-05-90$ & \\
\hline MBB 61C & 106091.1 & 55406.6 & $180.1-174.5$ & 314.9 & 317.6 & 143.1 & $4^{n}$ PVC & $04-14-87$ & \\
\hline MSB 61D & 106094.6 & 55390.6 & 234.8-214.9 & 316.7 & 318.1 & 103.2 & $4^{\prime \prime}$ PVC & $08-06-87$ & \\
\hline MSB 62B & 101865.3 & 47906.8 & 141.0 .136 .3 & 347.0 & 349.1 & 212.8 & $4^{\prime \prime}$ PVC & $11-16-90$ & \\
\hline MBB 62C ${ }^{2}$ & 101857.2 & 47895.0 & $190.0-185.2$ & 347.0 & 349.1 & 163.9 & 4" PVC & $11-26-90$ & \\
\hline MBB 62D 1 & 101849.0 & 47882.9 & 231.9-212.4 & 347.4 & 349.5 & 137.1 & 4" PVC & $11-26-90$ & \\
\hline MSB 63B ${ }^{1}$ & 101184.4 & 47861.0 & 140.9-136.2 & 344.9 & 347.0 & 210.8 & $4^{n}$ PVC & $03-21-90$ & \\
\hline MSB 63C ${ }^{1}$ & 101174.6 & 47849.2 & 196.8-191.1 & 344.8 & 347.1 & 156.0 & 4" PVC & $03-23-90$ & \\
\hline MSB 63D & 101165.2 & 47837.4 & 232.8-212.8 & M44.8 & 346.8 & 134.0 & $4^{n}$ PVC & $07-23-90$ & \\
\hline MSB 64B & 101831.0 & 46579.7 & $124.6-119.9$ & 946.6 & 348.7 & 228.8 & 4" PVC & $02-28-90$ & \\
\hline MSB 64C & 101842.9 & 46589.2 & 181.6.176.8 & 346.5 & 348.7 & 171.9 & $4^{n}$ PVC & $03-12-90$ & \\
\hline MSB 64D' & 101854.8 & 46598.5 & 230.6-210.6 & 347.0 & 349.0 & 138.6 & $4^{n}$ PVC & $07-23-90$ & \\
\hline MSB 6ID & 101916.6 & 49413.7 & 244.2-224.7 & 347.4 & 349.6 & 124.8 & $4^{n}$ PVC & $10-12-88$ & \\
\hline MSB 66B & 105842.0 & 51064.6 & $144.0-139.3$ & 381.5 & 383.5 & 244.2 & 4" PVC & $11-17.88$ & \\
\hline MBB 66C & 105842.1 & 51053.5 & $171.0-166.3$ & 381.6 & 383.6 & 217.2 & $4^{n}$ PVC & $11-28.88$ & \\
\hline MSB 66D & 105841.8 & 61044.0 & 249.5-219.4 & 381.6 & 383.3 & 163.9 & $4^{n}$ PVC & $12-21-88$ & \\
\hline MBB 66TA & 105842.6 & 51096.7 & 35.7. 31.0 & 380.7 & 382.8 & 351.8 & $* *$ & $12.21-88$ & \\
\hline MBB 67B & 106842.0 & 61989.6 & 134.9-130.1 & 362.7 & 366.1 & 235.0 & $4^{n}$ PVC & $11-09-88$ & \\
\hline MSB 67C & 106819.8 & 51988.6 & $174.9-170.1$ & 362.7 & 364.8 & 194.7 & $4^{n}$ PVC & 11.11 .88 & \\
\hline MBB 67D & 106830.7 & 51971.6 & $241.0-221.5$ & 363.1 & 365.0 & 143.5 & 4" PVC & $11-11-88$ & \\
\hline MSB 68B & 106744.9 & 52308.5 & $133.0-128.3$ & 365.0 & 356.9 & 228.6 & $4^{n}$ PVC & $12-01-88$ & \\
\hline MSB 68C & 106730.5 & 52304.9 & $171.7-167.0$ & 354.7 & 966.7 & 189.7 & $4^{*}$ PVC & $12-14-88$ & \\
\hline MSB 68D & 106741.4 & 62293.6 & 239.9-220.4 & 354.9 & 957.0 & 136.6 & $4^{n}$ PVC & $12-06-88$ & \\
\hline MBB 69B & 107776.1 & 52432.9 & $144.7 \cdot 140.0$ & 379.7 & 381.7 & 241.7 & $4^{n}$ PVC & $01-26-89$ & \\
\hline MBB 69C & 107780.1 & 62447.6 & 176.7.171.0 & 379.7 & 381.8 & 210.8 & $4^{\prime \prime}$ PVC & $01-31-89$ & \\
\hline MSB 69D & 107784.3 & 52462.0 & 239.9-220.4 & 379.9 & 382.2 & 161.8 & $4^{n}$ PVC & $02.01-89$ & \\
\hline MSB 69TA & 107772.5 & 52418.4 & $80.4-74.7$ & 379.4 & 381.6 & 306.3 & $* *$ & $01-26-89$ & \\
\hline MBB 70C & 101785.2 & 45012.0 & 179.2-174.5 & 360.2 & 362.2 & 187.7 & $4^{\prime \prime}$ PVC & $08-29-88$ & \\
\hline MBB 70D & 101781.8 & 44997.3 & 228.6-208.6 & 360.6 & 362.5 & 154.0 & 4" PVC & $07-23-90$ & \\
\hline MBB 71B & 103801.6 & 44054.7 & 136.2-131.4 & 343.2 & 346.1 & 213.7 & $4^{\prime \prime}$ PVC & $10-28-88$ & \\
\hline MSB 72B & 96387.6 & 48350.3 & $156.6-151.9$ & 326.6 & 328.2 & 176.3 & $4^{\prime \prime}$ PVC & 01.31 .89 & \\
\hline MSB 73B & 99270.3 & 45694.0 & 136.2-131.6 & 338.2 & 340.4 & 208.9 & $4^{n}$ PVC & $02-08-89$ & \\
\hline MSB 74B & 99197.4 & 50443.2 & $147.5-142.8$ & 312.6 & 314.5 & 171.7 & 4" PVC & 11.01 .89 & \\
\hline MSB 74C & 99191.1 & 50457.1 & $177.8-173.1$ & 312.8 & 316.0 & 141.9 & $4^{n}$.PVC & 11.09 .89 & \\
\hline MSB 74D & 99186.3 & 50469.7 & 237.1-217.1 & 313.1 & 315.1 & 98.0 & 4" PVC & $11-09-89$ & \\
\hline MSB 75B & 98937.4 & 48875.5 & $161.6-156.8$ & 324.6 & 326.7 & 169.9 & 4" PVC & 11.17 .89 & \\
\hline MSB 75C & 98942.3 & 48859.7 & 193.4-188.7 & 325.4 & 327.5 & 138.8 & $4^{n}$ PVC & $11.21-89$ & \\
\hline MSB 76C & 103061.6 & 45344.0 & 186.8-182.1 & 350.8 & 352.8 & 170.7 & 4" PVC & 02-06-90 & \\
\hline MSB 77B & 107065.8 & 54217.4 & $145.6-140.9$ & 355.6 & 367.7 & 216.8 & 4" PVC & 07.10 .90 & \\
\hline MSB 77C & 107078.3 & 54225.9 & 173.7.169.0 & 355.7 & 357.7 & 188.7 & 4" PVC & $07.10-90$ & \\
\hline MSB 77D & 107090.7 & 54233.4 & 236.7.216.7 & 355.7 & 357.8 & 141.1 & $4^{n}$ PVC & $07-12-90$ & \\
\hline MSB 7TTA & 107053.8 & 54208.9 & 83.4 78.1 & 355.4 & 357.4 & 279.3 & $* *$ & $07.05-90$ & \\
\hline MSB 78D & 103643.8 & 45482.2 & 225.9-206.5 & 361.5 & 363.6 & 157.1 & $4^{\prime \prime}$ PVC & $10-23-90$ & \\
\hline
\end{tabular}


MSB WELL SERIES (cont.)

\begin{tabular}{|c|c|c|c|c|c|c|c|c|}
\hline \multirow[b]{2}{*}{ Well ID } & \multicolumn{2}{|c|}{ gins Coordinates } & \multicolumn{3}{|c|}{ Elevations (ft MSL) } & \multirow{2}{*}{$\begin{array}{l}\text { Bff. Well } \\
\text { Depth (ft) }\end{array}$} & \multirow[b]{2}{*}{ Casing } & \multirow[b]{2}{*}{ Inotalled } \\
\hline & North & Fast & Ecreen 7one & Grnd. & Caring & & & \\
\hline MAB 79B & $\$ 2296.9$ & 47300.2 & $140.9-136.2$ & 346.9 & 348.0 & 211.8 & $4^{*}$ PVC & $12-04-89$ \\
\hline MSB 79C & 99290.2 & 47286.8 & $199.6-194.9$ & 345.6 & 347.8 & 152.9 & 4" PVC & $01-09-90$ \\
\hline MSB 81B & 103762.7 & 66230.4 & $146.3-141.6$ & 266.3 & 267.2 & 125.6 & 4" PVC & $06-04-90$ \\
\hline MSB 82A & 107529.6 & 51978.4 & $126.3-121.6$ & 372.3 & 374.6 & 252.9 & 4" PVC & $08-14-90$ \\
\hline MBB 82B & 107533.4 & 61993.3 & 148.4143 .7 & 372.4 & 374.4 & 230.7 & $4^{n}$ PVC & $08-14-90$ \\
\hline MSB 82C & 107621.9 & 51949.4 & 177.9-173.2 & 371.9 & 374.0 & 200.8 & 4" PVC & $05-15-89$ \\
\hline MBB $82 D$ & 107618.1 & 61934.6 & 237.1-217.0 & 371.6 & 373.7 & 166.7 & & $0808-90$ \\
\hline MBB 82TA & 107525.7 & 51984.2 & 94.0 .88 .6 & 372.0 & 373.8 & 285.2 & $* *$ & 08-06-90 \\
\hline MBB 83B & 108426.7 & 52421.4 & 146.5.141.8 & $\mathbf{3 7 0 . 0}$ & 372.0 & 290.2 & 4" PVC & $12-17.90$ \\
\hline MBB 8SC & 108406.3 & 52384.7 & $185.0-178.2$ & 970.0 & 372.1 & 199.9 & 4" PVC & $05-10-89$ \\
\hline MBB 89D & 108418.4 & 52991.2 & 256.9-216.9 & $\$ 69.6$ & 371.7 & 164.8 & & $11-16-90$ \\
\hline MBB BSTA & 108416.3 & 52410.9 & $75.8 \quad 70.3$ & $\$ 69.8$ & 971.8 & 301.5 & $* *$ & $12-14-90$ \\
\hline MBB $84 \mathrm{C}$ & 108967.9 & 61973.7 & $198.0-190.3$ & 360.0 & 362.0 & 171.7 & & $06-04-89$ \\
\hline MBB 86B & 107827.0 & 63122.7 & $137.8-133.1$ & 378.8 & 380.8 & 247.7 & vC & $01-24-80$ \\
\hline MBB 85C & 107835.2 & 53161.4 & 174.1-169.3 & 379.1 & 381.4 & 212.1 & & $06-19-89$ \\
\hline MBB 85D & 107822.8 & 63108.8 & 236.8-216.7 & 379.3 & 381.4 & 164.7 & VC & $08-23-90$ \\
\hline MBB 86TA & 107831.2 & 63137.2 & 88.7- 83.3 & 378.9 & 381.0 & 297.7 & $4^{\prime \prime}$ Btoel & $01-02-90$ \\
\hline MBB 86C & 108600.4 & 63560.6 & 165.2-160.6 & 356.2 & 357.4 & 196.9 & 4" PVC & $10-12-89$ \\
\hline
\end{tabular}

Notes: During initial development of MSBB 69D, formation eediments and gravel pack reeped into the well, indicating that the upper acreen or lower caeing had broken. A 2-in. PVC casing was inotallod ineide the 4in. casing to salvage the well.

According to Geraghty \& Miller (1986), three welle were abandoned and replaced during the inotallation of monitoring wells in the $A$ and $M$ areas. The original 1988 s.TA was abandoned after grout entered the screen zone. The original MSB STIA was abandoned "as a recult of poevible equeezing clays andar driller's error." The original MISB STD was abandoned due to the probability of grout entoring the scroen zone.

The Screon Zone Elevations in parentheses are in feet bolow M8L.

${ }^{2}$ Point-of-compliance well for the M-Area HWMT, comprising the M-Area Bottling Bavin (904-61G), Lost Lake (904-112G), an overflow ditch, and a ecopage aroa.

${ }^{2}$ Background woll for the M-Area HWMT, compriving the M-Area Bettling Basin (904-61G), Lost Lake (904-112G), an overflow ditch, and a seepage area.

- Plume definition welle aleo required by the 8RS Hazardous Waste Permit.

a The inatalled dates for these welle are the dates on the casing tally whoets in Geraghty \& Millor (1986).

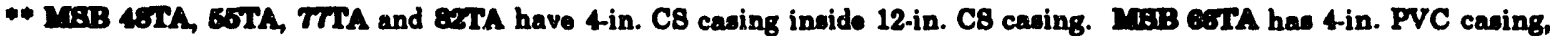
inside 8-in. C8 intermediate casing, inside 12-in. C8 surface casing. MBB ceTA and 85TA have 4-in. PVC casing insido 12-in. steol caving. All ecreens are 88.

MBSB 29DD and 4SDD are piezometera. 


\section{MVC WELL SERIES}

Description: M-Area Vadose Zone Vapor Probes

Bldg. Number:

Map: Figure A-18

\begin{tabular}{|c|c|c|c|c|c|c|c|c|c|}
\hline \multirow[b]{2}{*}{ Well ID } & \multicolumn{2}{|c|}{ 8R8 Coordinates } & \multicolumn{3}{|c|}{ Elevations (f MSL) } & \multirow{2}{*}{$\begin{array}{l}\text { Eff. Well } \\
\text { Depth (ft) }\end{array}$} & \multirow[b]{2}{*}{ Casing } & \multirow[b]{2}{*}{ Installed. } & \multirow[b]{2}{*}{ Abandoned } \\
\hline & North & East & Screen Zone & Grnd. & Casing & & & & \\
\hline MVC IA & 104275.2 & 49331.6 & 245.9.240.9 & 371.0 & 373.0 & 132.1 & 1" PVC & $10-04-90$ & \\
\hline MVC 1B & 104275.2 & 49331.6 & $301.0-296.0$ & 371.0 & 373.0 & 77.0 & 1" PVC & 10.05 .90 & \\
\hline MVC 1C & 104275.2 & 49331.6 & $321.0-316.0$ & 371.0 & 373.0 & 57.0 & $1^{*} \mathrm{PVC}$ & $10-05-90$ & \\
\hline MVC 2A & 101970.0 & 50060.2 & 244.5-239.6 & 346.6 & 348.6 & 109.1 & 1" PVC & $09.13-90$ & \\
\hline MVC 2B & 101970.0 & 50060.2 & 278.7.273.7 & 346.5 & 348.6 & 74.9 & 1" PVC & $09-13-90$ & \\
\hline MVC 2C & 101970.0 & 50060.2 & $302.0-297.0$ & 346.6 & 348.6 & 51.6 & $1^{n} \mathrm{PVC}$ & $09-17-90$ & \\
\hline MVC 3A & 101833.9 & 50120.4 & 240.7-235.7 & 342.7 & 344.7 & 109.0 & 1" PVC & $09-21.90$ & \\
\hline MVC 3B & 101833.9 & 60120.4 & 277.7-272.7 & $\mathbf{3 4 2 . 7}$ & 344.7 & 72.0 & 1" PVC & $09-24-90$ & \\
\hline MVC 3C & 101833.9 & 50120.4 & 294.7.289.7 & 342.7 & 344.7 & 65.0 & 1" PVC & $09.24-90$ & \\
\hline
\end{tabular}

Notes: Each cluster in the MVC geries is comprised of one borehole with threv vapor probes installed inside. The "A" wells are deep probes, the "B" wells are intermediate probes, and the " $\mathrm{C}$ " welle are shallow probes. All screens are 88.

\section{MVE WELL SERIES}

Description: M-Area Vadose Zone Extraction Wells

Bldg. Number:

Map: Figure A-18

\begin{tabular}{|c|c|c|c|c|c|c|c|c|c|}
\hline \multirow[b]{2}{*}{ Well IP } & \multicolumn{2}{|c|}{ SRS Coordinates } & \multicolumn{3}{|c|}{ Blovations (At MSL) } & \multirow{2}{*}{$\begin{array}{l}\text { Rff. Well } \\
\text { Depth (ft) }\end{array}$} & \multirow[b]{2}{*}{ Casing } & \multirow[b]{2}{*}{ Installed } & \multirow[b]{2}{*}{ Abandoned } \\
\hline & North & Fast & Ecreen Zone & Grnd. & Caning & & & & \\
\hline & 104319.1 & 49357.2 & 356.2-235.7 & 970.7 & 372.8 & 137.1 & $4^{n}$ Stoel & $10-31-90$ & \\
\hline & 104318.5 & 49307.2 & 341.8.237.8 & 370.6 & 372.9 & 135.1 & 4" Bteel & $10-18-90$ & \\
\hline & 104407.0 & 49392.2 & $303.0-202.5$ & 336.4 & 371.9 & 169.4 & 4" Steel & 11.02 .90 & \\
\hline v & 102009.1 & 49984.6 & 293.9-232.5 & $\mathbf{3 4 7 . 5}$ & 350.0 & 117.5 & $4^{\prime \prime} \mathrm{CS}$ & 09.19 .90 & \\
\hline
\end{tabular}

\section{MWD WELL SERIES}

Description: Potential Hazardous Waste/Mixed Waste Disposal Facility Bldg. Number: Not applicable

Map: Figure A-23

\begin{tabular}{|c|c|c|c|c|c|c|c|c|c|}
\hline \multirow[b]{2}{*}{ Well II } & \multicolumn{2}{|c|}{ BRS Coordinates } & \multicolumn{3}{|c|}{ Elevations (ft MSL) } & Eff. Well & \multirow[b]{2}{*}{ Casing } & \multirow[b]{2}{*}{ Installed } & \multirow[b]{2}{*}{ Abandoned } \\
\hline & North & East & Screen Zone & Grnd. & Casing & Depth (ft) & & & \\
\hline MWD 1A & 69592.8 & 75121.9 & 122.5- 57.4 & 327.5 & 329.5 & 272.1 & 4" PVC & $05-26-88$ & \\
\hline MWD 1B & 69611.8 & 75114.1 & $133.8-131.6$ & 327.4 & 329.6 & 198.0 & $4^{n}$ PVC & $05-18-88$ & \\
\hline MWD 1C & 69607.6 & 75127.8 & 207.0-172.0 & 327.0 & 328.6 & 171.6 & 4" PVC & $04-11.88$ & \\
\hline & & & $167.0-157.0$ & & & & & & \\
\hline MWD $1 D$ & 69598.7 & 75108.1 & 272.9.227.8 & 327.9 & 330.0 & 102.2 & 4" PVC & $04-06.88$ & \\
\hline MWD 2A & 69768.8 & 75241.1 & 122.7. 57.5 & 322.7 & 324.4 & 266.9 & $4^{n}$ PVC & $04-26.88$ & \\
\hline MWD 2C & 69771.5 & 75255.5 & 202.5-152.4 & 322.5 & 324.3 & 171.9 & $4^{n}$ PVC & $04-14-88$ & \\
\hline
\end{tabular}

(ceries continued) 
MWD WELL SERIES (cont.)

\begin{tabular}{|c|c|c|c|c|c|c|c|c|c|}
\hline \multirow[b]{2}{*}{ Well ID } & \multicolumn{2}{|c|}{ 8RS Coordinates } & \multicolumn{3}{|c|}{ Elevations (ft MSL) } & \multirow{2}{*}{$\begin{array}{l}\text { Eff. Well } \\
\text { Depth (t) }\end{array}$} & \multirow[b]{2}{*}{ Casing } & \multirow[b]{2}{*}{ Installed } & \multirow[b]{2}{*}{ Abandoned } \\
\hline & North & East & Screen Zone & Grnd. & Casing & & & & \\
\hline MWD 2D & 69761.4 & 75266.6 & 267.6-247.5 & 322.5 & 324.6 & 77.1 & $4^{n}$ PVC & $05-09-88$ & \\
\hline MWD SA & 69839.4 & 74756.6 & 123.7.103.7 & 328.7 & 330.8 & 227.1 & $4^{n}$ PVC & $05-10-88$ & \\
\hline MWD $\mathbf{5 A}$ & 69234.7 & 75490.8 & 122.0- 66.9 & 322.6 & 324.6 & 267.7 & $4^{\prime \prime}$ PVC & $05-06-88$ & \\
\hline MWD $6 \mathrm{C}$ & 69226.8 & 75800.2 & 202.6-152.6 & 322.8 & 925.1 & 172.6 & $4^{n}$ PVC & $04-25-88$ & \\
\hline MWD 6D & 69220.3 & 75486.6 & 269.6-249.6 & 323.0 & 324.9 & 75.4 & $4^{n}$ PVC & $04-01.88$ & \\
\hline MWD 8 & 70074.0 & 75101.6 & 273.7-253.7 & 323.7 & 325.8 & 72.1 & $4^{\prime \prime}$ PVC & $09-30-88$ & \\
\hline MWD 9 & 68948.9 & 75201.0 & $260.6-240.6$ & 320.6 & 322.5 & 81.9 & 4" PVC & $03-31.88$ & \\
\hline MWD 10 & 69508.7 & 75085.6 & 205.2-169.8 & 328.9 & 330.9 & 171.1 & 6" PVC & $06-03-88$ & \\
\hline MWD 11 & 69475.5 & 75087.9 & 124.463 .9 & 328.9 & 330.8 & 266.9 & 6" PVC & $06-17.88$ & \\
\hline
\end{tabular}

Notes: MWD $1 \mathrm{C}$ hese $5 \mathrm{ft}$ of casing set between 172.0 and $167.0 \mathrm{ft} \mathrm{MSL}$ and a screen above $172.0 \mathrm{ft}$ and one below $167.0 \mathrm{ft}$.

MWD2D and 11 were originally constructed in other locations. MWD 2D was abandoned because the screen was broken. MWD 11 was abandoned because the casing joint failed during the second atage of grouting. These firgt wells were never accepted by Du Pont. The data above are for the wells that were accepted as replacements by Du Pont.

N 13 WELL SERIES (see NPN WELL SERIES)

N 91 Well (see K WELL SERIES)

NBG WELL SERIES

Description: Wells between the F-Area Canyon Building and the Naval Fuel Material Facility Bldg. Number: 247-F

Map: Figure A-12

\begin{tabular}{|c|c|c|c|c|c|c|c|c|c|}
\hline \multirow[b]{2}{*}{ Well ID } & \multicolumn{2}{|c|}{ BR8 Coordinates } & \multicolumn{3}{|c|}{ Hevations (f MSL) } & \multirow{2}{*}{$\begin{array}{l}\text { Eff. Well } \\
\text { Depth (t) }\end{array}$} & \multirow[b]{2}{*}{ Casing } & \multirow[b]{2}{*}{ Inotalled } & \multirow[b]{2}{*}{ Abandoned } \\
\hline & North & Past & Brreen 7ane & Grand. & Casing & & & & \\
\hline NBG 1 & 79900.4 & 53879.3 & 232.3-200.9 & 309.6 & 311.6 & 110.6 & 4" PVC & $03-26-86$ & \\
\hline NBG 2 & 79099.8 & 53958.4 & 239.6-203.6 & 310.6 & 312.7 & 109.1 & $4^{n}$ PVC & $04-04-88$ & \\
\hline NBG 3 & 78989.6 & 54068.1 & 233.5-202.1 & 310.6 & 312.4 & 110.3 & 4" PVC & $03-24-86$ & \\
\hline NBG 4 & 78942.1 & 54329.2 & 227.6.196.1 & 304.5 & 308.5 & 110.4 & 4" PVC & $04-01-88$ & \\
\hline NBG 6 & 78943.4 & 54516.6 & $226.4-194.9$ & 301.4 & 303.5 & 108.6 & 4" PVC & $03-28-86$ & \\
\hline
\end{tabular}




\section{NPM WELL SERIES}

Description: Preliminary Hydrogeologic Evaluation of Potential New Production Reactor Site Characterization Wells

Bldg. Number: Not applicable

Map: Figure A-21

\begin{tabular}{|c|c|c|c|c|c|c|c|c|c|}
\hline \multirow[b]{2}{*}{ Well ID } & \multicolumn{2}{|c|}{ SRS Coordinateg } & \multicolumn{3}{|c|}{ Elevations (ft MSL) } & \multirow{2}{*}{$\begin{array}{l}\text { Eff. Well } \\
\text { Depth (ft) }\end{array}$} & \multirow[b]{2}{*}{ Casing } & \multirow[b]{2}{*}{ Inatalled } & \multirow[b]{2}{*}{ Abandoned } \\
\hline & North & Eagt & Screen Zone & Grnd. & Casing & & & & \\
\hline NPM 1 & 62153.4 & 56851.6 & 277.1-257.1 & 303.8 & 306.1 & 49.0 & 4" PVC & 05.01 .89 & \\
\hline NPM $1 A$ & 62170.7 & 56819.9 & $19.3 \quad 9.3$ & 303.7 & 305.9 & 296.6 & $4^{n}$ PVC & $05-26-89$ & \\
\hline NPM 2 & 63056.8 & 58252.0 & $264.2-244.2$ & 334.9 & 397.1 & 92.9 & $4^{n}$ PVC & 05.17 .89 & \\
\hline NPM 3 & 62109.2 & 55417.6 & $267.2-247.2$ & 333.9 & 336.3 & 89.1 & $4^{n}$ PVC & $05-24-89$ & \\
\hline NPM 4 & 60883.2 & 57215.0 & 276.7-256.7 & 311.6 & 313.8 & 67.1 & $4^{n}$ PVC & 05.11 .89 & \\
\hline NPM 4DD & 60893.1 & 57218.8 & $306.4-296.4$ & 311.3 & 313.6 & 17.2 & $4^{n} \mathrm{PVC}$ & 05.11 .89 & \\
\hline
\end{tabular}

Notes: This series was originally referred to as the $\mathbf{M} 12$ Series.

\section{NPN WELL SERIES}

Description: Preliminary Hydrogeologic Evaluation of Potential New Production Reactor Site Characterization Wells

Bldg. Number: Not app! icable

Map: Figure A-23

\begin{tabular}{|c|c|c|c|c|c|c|c|c|c|}
\hline \multirow[b]{2}{*}{ Well ID } & \multicolumn{2}{|c|}{ gRS Coordinateg } & \multicolumn{3}{|c|}{ Elevations (ft MSL) } & \multirow{2}{*}{$\begin{array}{l}\text { Eff. Well } \\
\text { Depth (ft) }\end{array}$} & \multirow[b]{2}{*}{ Casing } & \multirow[b]{2}{*}{ Installed } & \multirow[b]{2}{*}{ Abandoned } \\
\hline & North & East & Screen Zone & Grnd. & Casing & & & & \\
\hline NPN 1 & 66661.4 & 70879.6 & $277.4-257.3$ & 335.0 & 337.2 & 79.9 & 4" PVC & $06-19-89$ & \\
\hline NPN 1A & 66632.1 & 70856.2 & 40.9. 30.9 & 335.9 & 338.1 & $\mathbf{5 0 7 . 2}$ & $4^{\prime \prime}$ PVC & $05-22-89$ & \\
\hline NPN 2 & 67394.1 & 72541.6 & 278.0-257.9 & 327.6 & 329.8 & 71.9 & $4^{n}$ PVC & $06-02-89$ & \\
\hline NPN 3 & 67989.8 & 70029.2 & $280.1-260.0$ & 316.2 & 317.4 & 67.4 & $4^{n}$ PVC & $06-02-89$ & \\
\hline NPN 4 & 65367.2 & 71021.8 & 285.6.265.4 & 305.1 & 307.3 & 41.9 & $4^{\prime \prime}$ PVC & $05-29-89$ & \\
\hline
\end{tabular}

Notes: This series was originally referred to as the N 13 Series.

\section{NTN WELL SERIES}

Description: Preliminary Hydrogeologic Evaluation of Potential New Production Reactor Site Characterization Wells

Bldg. Number: Not applicable

Map: Figures A-3 and A-4

\begin{tabular}{|c|c|c|c|c|c|c|c|c|c|}
\hline \multirow[b]{2}{*}{ Well ID } & \multicolumn{2}{|c|}{ BRS Coordinates } & \multicolumn{3}{|c|}{ Elevations (ft MSL) } & \multirow{2}{*}{$\begin{array}{l}\text { Eff. Well } \\
\text { Depth (ft) }\end{array}$} & \multirow[b]{2}{*}{ Casing } & \multirow[b]{2}{*}{ Ingtalled } & \multirow[b]{2}{*}{ Abandoned } \\
\hline & North & East & Screen Zone & Grnd. & Casing & & & & \\
\hline NTN & 56993.7 & 45562.3 & $232.4-212.4$ & 257.8 & 259.9 & 47.5 & 4" PVC & $05-02.89$ & \\
\hline
\end{tabular}


NTN WELL SERIES (cont.)

\begin{tabular}{|c|c|c|c|c|c|c|c|c|}
\hline & BR8 Coordinates & Plovations (f & MSL) & & Eff. Well & & & \\
\hline Well ID & North East & Ecreen Zone & Grnd. & Casing & Depth (ft) & Casing & Installed & Abandoned \\
\hline $\begin{array}{ll}\text { TIN } & 1 A \\
T N & 2\end{array}$ & $\begin{array}{ll}57015.7 & 46553.2 \\
57935.5 & 46735.1\end{array}$ & $\begin{array}{l}\text { (41.6-51.6) } \\
227.2-207.2\end{array}$ & $\begin{array}{l}258.4 \\
254.7\end{array}$ & $\begin{array}{l}260.5 \\
256.9\end{array}$ & $\begin{array}{r}312.1 \\
49.7\end{array}$ & $\begin{array}{l}4^{n} \text { PVC } \\
4^{n} \text { PVC }\end{array}$ & $\begin{array}{l}06-08-89 \\
05-03-89\end{array}$ & \\
\hline
\end{tabular}

Notes: This series was originally referred to as the $\mathbf{T} 18 \mathrm{~N}$ Series.

The Screen Zone Elevation in parenthesis is in feet below MBL.

\section{NTS WELL SERIES}

Description: Preliminary Hydrogeologic Evaluation of Potential New Production Reactor Site Characterization Wells

Bldg. Number: Not applicable

Map: Figure A-4

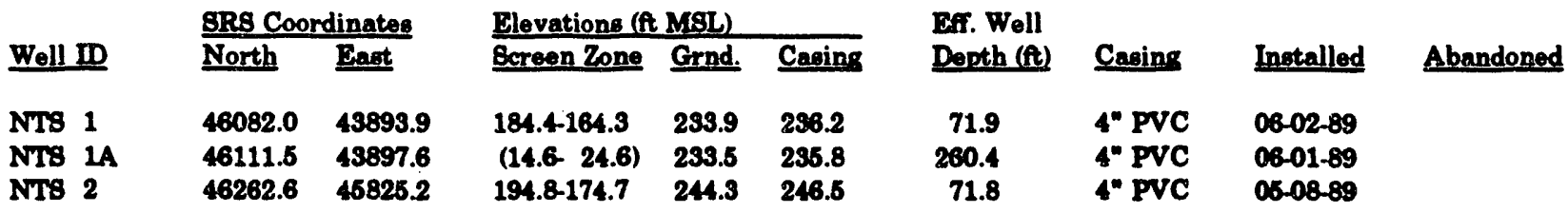

Notes: This ceries was originally referred to as the $T 188$ Series.

The Screen Zone Elevation in parentheris is in foot below MSL.

\section{NTW WELL SERIES}

Description: Preliminary Hydrogeologic Evaluation of Potential New Production Reactor Site Characterization Wells

Bldg. Number: Not applicable

Map: Figure A-s

\begin{tabular}{|c|c|c|c|c|c|c|c|c|c|}
\hline \multirow[b]{2}{*}{ Well ID } & \multicolumn{2}{|c|}{ SRS Coordinates } & \multicolumn{3}{|c|}{ Elevations ( $f$ MSL) } & \multirow{2}{*}{$\begin{array}{l}\text { Efr. Woll } \\
\text { Depth (A) }\end{array}$} & \multirow[b]{2}{*}{ Casing } & \multirow[b]{2}{*}{ Ingtalled } & \multirow[b]{2}{*}{ Abandoner } \\
\hline & North & Eagt & Screen Zone & Grnd. & Casing & & & & \\
\hline NIW 1 & 48776.5 & 40267.7 & 188.8-168.9 & 244.9 & 247.0 & 78.1 & $4^{n}$ PVC & $12-16-89$ & \\
\hline NIW IA & 48773.1 & 40276.2 & (10.6-20.6) & 244.4 & 246.6 & 287.2 & & $12-16-89$ & \\
\hline NIW 2 & 49309.3 & 39353.2 & 191.6-171.5 & 243.1 & 245.4 & 73.9 & $4^{\prime \prime}$ PVC & $12-07-89$ & \\
\hline NIW 3 & 50040.0 & 41208.7 & $196.6-176.7$ & 259.5 & 261.7 & 85.0 & $4^{n}$ PVC & $11-29-89$ & \\
\hline NTW 4 & 48636.3 & 41678.2 & $185.8-166.0$ & 226.2 & 228.5 & 62.5 & $4^{n}$ PVC & $11-30-89$ & \\
\hline
\end{tabular}

Notes: This series was originally referred to as the $T 18 \mathrm{~W}$ Series.

The Screen Zone Elevation in parenthesis is in feet below MBL. 


\section{OBS Wells}

Description: Unofficial wells in Z Area

Bldg. Number: Not applicable

Map: Figure A-16

\begin{tabular}{|c|c|c|c|c|c|c|c|c|}
\hline \multirow[b]{2}{*}{ Well D } & \multicolumn{2}{|c|}{ BRS Coordinates } & \multicolumn{2}{|l|}{ Elevations (ft MSL) } & \multirow{2}{*}{$\begin{array}{l}\text { Eff. Well } \\
\text { Depth (ft) }\end{array}$} & \multirow[b]{2}{*}{ Casing } & \multirow[b]{2}{*}{ Installed } & \multirow[b]{2}{*}{ Abandoned } \\
\hline & North & East & Screen Zone Grnd. & Casing & & & & \\
\hline OBS 1 & 78340 & 65700 & & & & & & \\
\hline OBS 2 & 78770 & 67510 & & & & & & \\
\hline OBS 3 & 77920 & 67540 & & & & & & \\
\hline $\begin{array}{ll}\text { OBS } 4 \\
\text { OBS } 5\end{array}$ & $\begin{array}{l}77840 \\
77320\end{array}$ & $\begin{array}{l}67620 \\
67480\end{array}$ & & & & $2^{n} \mathrm{Al}$ & & $04-02-87$ \\
\hline
\end{tabular}

Notes: The identification of these wells comes from the table, "DWPF Borehole and Piezometer Abandonments," in Heckrotte (1987). The OBs designation is unofficial. The table states that OBg 1, 2, 3, and 6 were not found. By inference this would mean that these wells were not officially abandoned. The eame table lists the above coordinates for OBS 4 , but indicates that they may be incorrect.

There has been some confusion in the past between 0832 and 8108 124. The abandonment Daily Activity Report for EDS 124 (5-19-87) вays that "SD8 124 was written on side of PVC casing but covered with paper label marked as OBS 2." It appears that OBS 2 and BDS 124 are not the same well. Soe $8 D 8124$ for further information.

\section{$P$ WELL SERIES}

Description: SRS Baseline Hydrogeologic Investigation Observation Well Clusters-see below for specific locations

Bldg. Number: See below

Map: Figure A-29

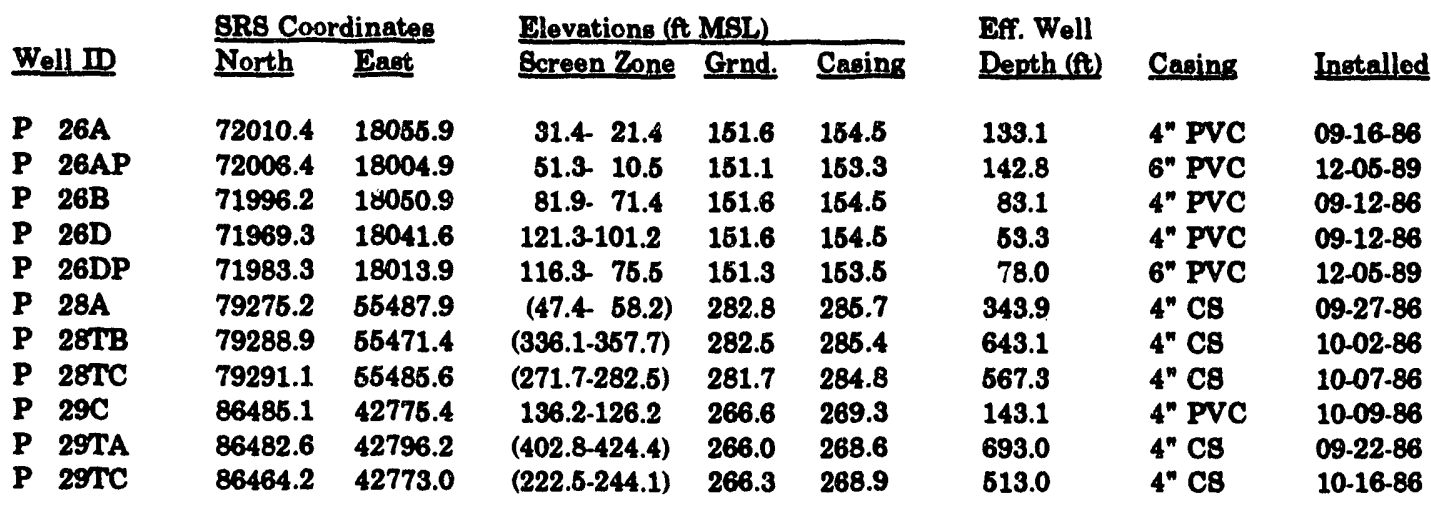

(series continued) 


\section{P SERIES (cont.)}

Location

B-Area Microbiology Wells

F-Area Microbiology Welle

TNX-Area Background Welle near the TNX Facility at the Northeast Intereection of SR8 Roads 3 and

$3-1$, approximately $160 \mathrm{ft}$ off Road 3

TNX-Area Pump Teat Well for the Congaree

Formation

TNX-Area Pump Teat Well for the Water Table
Facility No.

Not applicable

Not applicable

704-T

Not applicable

Not applicable
Cluster/Well

P 29

P 28

P 26

P 26AP

P 26DP

\section{PAC WELL SERIES}

Description: P-Area Acid/Caustic Basin Bldg. Number: 904-78G

Map: Figure A-6

\begin{tabular}{|c|c|c|c|c|c|c|c|c|c|}
\hline \multirow[b]{2}{*}{ Well ID } & \multicolumn{2}{|c|}{ gRS Coordinates } & \multicolumn{3}{|c|}{ Plevations (f MSI) } & \multirow{2}{*}{$\begin{array}{l}\text { Eff. Well } \\
\text { Depth (t) }\end{array}$} & \multirow[b]{2}{*}{ Casing } & \multirow[b]{2}{*}{ Installed } & \multirow[b]{2}{*}{ Abandoned } \\
\hline & North & East & Sereen Zope & Grnd. & Casing & & & & \\
\hline & 43643.3 & 66763.4 & 283.9-253.9 & 293.9 & 295.9 & 42.0 & $4^{*} \mathrm{PVC}$ & $11-03-83$ & \\
\hline PAC 2 & 49627.7 & 66980.9 & 277.9-247.9 & 282.9 & 284.8 & 36.9 & $4^{*}$ PVC & $11-04-83$ & \\
\hline PAC 3 & 49585.6 & 66881.4 & 282.9-252.9 & 287.9 & 289.9 & 37.0 & 4" PVC & $11-03-83$ & \\
\hline PAC 4 & 43496.4 & 66863.2 & $280.6-250.6$ & 289.6 & 291.6 & 41.0 & 4" PVC & $07-06-84$ & \\
\hline PAC 5 & 49661.7 & 68907.1 & 275.1-255.1 & 287.1 & 289.3 & 34.2 & $4^{\prime \prime}$ PVC & $09-22-88$ & \\
\hline PAC 6 & 43580.1 & 66894.7 & $276.2-256.2$ & 287.2 & 289.4 & 34.2 & $4^{n}$ PVC & $09-29-88$ & \\
\hline
\end{tabular}

\section{PCB WELL SERIES}

- Description: P-Area Coal Pile Runoff Containment Basin Bldg. Number: 189-P

Map: Figure A-6

\begin{tabular}{|c|c|c|c|c|c|}
\hline \multirow[b]{2}{*}{ Well ID } & \multicolumn{2}{|c|}{ BRS Coordinates } & \multicolumn{3}{|c|}{ Elevations ( $\mathrm{A}$ MSL) } \\
\hline & North & East & Screen Rone & Grnd. & Casing \\
\hline CB 1 & 41998.6 & 65066.5 & $276.1-256.1$ & 903.6 & 305.6 \\
\hline PCB 1A & 41988.2 & 65070.6 & $293.5-263.5$ & 303.5 & 305.5 \\
\hline PCB 2 & 41823.2 & 64872.2 & $290.5-270.6$ & 302.8 & 304.8 \\
\hline PCB 2A & 41821.4 & 64891.4 & $287.8-257.8$ & 802.8 & 304.9 \\
\hline PCB 9 & 42021.3 & 84705.9 & 293.4-273.4 & 301.7 & 908.7 \\
\hline PCB 9A & 42036.0 & 64706.3 & 292.7-262.7 & 302.7 & 304.6 \\
\hline PCB 4 & 42175.6 & 64884.5 & $294.8-274.8$ & 307.1 & 309.1 \\
\hline PCB $4 A$ & 42171.0 & 64901.4 & 292.9-262.9 & 307.9 & 309.6 \\
\hline
\end{tabular}

Bff. Well

Depth (ft) Casing Installed

$4^{n}$ Stoel $03-31-80$

4 PVC 02-16-84

4 " Btoel 04-01-80

4" PVC 12-17-413

4" Btoel 04-0s-80

4" PVC 09-05-84

4" Steol 04-02-80

$4^{n}$ PVC 02-16-84

Abandoned
$01-01-84^{*}$
$01-01-84 *$
$03-06-84$
$01-01-84 *$

Abandoned

$01-01-84$ 


\section{PDB WELL SERIES}

Description: P-Area Disassembly Basin

Bldg. Number: 105-P

Map: Figure A.6

\begin{tabular}{|c|c|c|c|c|c|c|c|c|c|}
\hline & GRS Coo & dinates & Elovations (f & MSL) & & Eff. Well & & & \\
\hline Well ID & North & East & Bcreen Zone & Grnd. & Casing & Depth (ft) & Casing & Installed & Abandoned \\
\hline $\begin{array}{ll}\text { PDB } & 2 \\
\text { PDB } & 3\end{array}$ & $\begin{array}{l}43513.1 \\
43542.2\end{array}$ & $\begin{array}{l}64743.1 \\
64998.2\end{array}$ & $\begin{array}{l}268.7-247.7 \\
269.1-248.1\end{array}$ & $\begin{array}{l}316.9 \\
317.1\end{array}$ & $\begin{array}{l}319.6 \\
319.6\end{array}$ & $\begin{array}{l}71.8 \\
71.4\end{array}$ & $\begin{array}{l}\text { 4" PVC } \\
\text { 4" PVC }\end{array}$ & $\begin{array}{l}08-25-86 \\
08-28-86\end{array}$ & \\
\hline
\end{tabular}

PRODUCTION WELLS (see DRE 5WW Well, FSB 1TA Well, HPT 1A Well, HSB 1TB Well, and PW WELL SERIES)

\section{PRP WELL SERIES}

Description: P-Area Burning/Rubble Pit

Bldg. Number: 131-P

Map: Figure A-6

\begin{tabular}{|c|c|c|c|c|c|c|c|c|c|}
\hline \multirow[b]{2}{*}{ Well ID } & \multicolumn{2}{|c|}{ SRS Coordinateg } & \multicolumn{3}{|c|}{ Plovations (At MSL) } & \multirow{2}{*}{$\begin{array}{l}\text { Eff. Well } \\
\text { Depth (ft) }\end{array}$} & \multirow[b]{2}{*}{ Capins } & \multirow[b]{2}{*}{ Installed } & \multirow[b]{2}{*}{ Abandoned } \\
\hline & North & East & Screen Zone & Grnd. & Caring & & & & \\
\hline PRP 1 & 45349.8 & 63032.7 & & & & & $4^{n}$ PVC & 10.11 .83 & $10-14-83$ \\
\hline PRP 1A & 45349.8 & 63032.7 & 262.9-232.9 & 282.9 & 284.6 & 51.7 & 4" PVC & $10-17-83$ & \\
\hline PRP 2 & 45389.5 & 69229.0 & 264.1-234.1 & 284.1 & 288.4 & 52.3 & $4^{\prime \prime} \mathrm{PVC}$ & $10-18-89$ & \\
\hline PRP 3 & 45200.7 & 63165.5 & $258.6-228.6$ & 278.6 & 280.7 & 52.1 & 4" PVC & $10.13-83$ & \\
\hline PRP 4 & 45268.9 & 63345.9 & $262.9-232.9$ & 282.9 & 284.7 & 51.8 & $4^{\prime \prime}$ PVC & $07-05.84$ & \\
\hline
\end{tabular}

Notes: According to the Well Construction Detaile, PRP 1 had to be redrilled because the casing would not go down. The Drilling Requests indicate that PRP $1 A$ was drilled in the same hole as PRP 1.

\section{PSB WELL SERIES}

Description: P-Area Reactor Seepage Basins

Bldg. Number: 904-61G, -62G, -63G

Map: Figure A-6

\begin{tabular}{|c|c|c|c|c|c|c|c|c|c|}
\hline \multirow[b]{2}{*}{ Well ID } & \multicolumn{2}{|c|}{ BRS Coordinates } & \multicolumn{3}{|c|}{ Elovations (A MSL) } & \multirow{2}{*}{$\begin{array}{l}\text { Eff. Well } \\
\text { Depth (ft) }\end{array}$} & \multirow[b]{2}{*}{ Casing } & \multirow[b]{2}{*}{ Installed } & \multirow[b]{2}{*}{ Abandoned } \\
\hline & North & East & Bcreen Zone & Grnd. & Casing & & & & \\
\hline PBB 1 & 43620.6 & 64155.0 & & & & & $4^{\prime \prime}$ Steel & $05-31-78$ & $04-01.84^{*}$ \\
\hline PBB 1A & 43619.3 & 64141.4 & 287.4-257.4 & 327.4 & 329.1 & 71.7 & $4^{n}$ PVC & $03-15-84$ & \\
\hline PGB 2 & 43606.6 & 63906.6 & & & & & $4^{n}$ Btoel & $06-01-78$ & $04-01-84 *$ \\
\hline PSB 2A & 43612.4 & 63916.5 & 287.2-257.2 & 322.2 & 323.7 & 66.5 & $4^{n}$ PVC & $03-16-84$ & \\
\hline PSB 3 & 43599.6 & 63605.2 & & & & & 4" Steel & $06-05-78$ & $04-01.84 *$ \\
\hline PBB 3A & 43599.8 & 63590.4 & 286.5-256.5 & 316.5 & 318.6 & 62.1 & 4" PVC & $03-19-84$ & \\
\hline PSB 4 & 43524.1 & 63346.8 & & & & & 4" Steel & $06-05-78$ & $04-01-84^{*}$ \\
\hline PBB 4A & 43534.2 & 63347.0 & $285.5-256.5$ & 310.5 & 312.5 & 57.0 & $4^{n}$ PVC & $03-20-84$ & \\
\hline
\end{tabular}

(eeries continued) 
PSB WELL SERIES (cont.)

\begin{tabular}{|c|c|c|c|c|c|c|c|c|c|}
\hline \multirow[b]{2}{*}{ Well ID } & \multicolumn{2}{|c|}{ SRB Coordinates } & \multicolumn{3}{|c|}{ Elevations (ft MSL) } & \multirow{2}{*}{$\begin{array}{l}\text { Eff. Well } \\
\text { Depth (ft) }\end{array}$} & \multirow[b]{2}{*}{ Casing } & \multirow[b]{2}{*}{ Installed } & \multirow[b]{2}{*}{ Abandoned } \\
\hline & North & East & Ecreen Zone & Grnd. & Casing & & & & \\
\hline PSB 5 & 43438.4 & 63619.4 & & & & & $4^{\prime \prime}$ Steel & $06-05-78$ & $04-01.84^{\star}$ \\
\hline PBB 5A & 43440.5 & 63606.5 & 292.3-262.3 & 317.3 & 319.3 & 57.0 & 4" PVC & $03-20-84$ & \\
\hline PSB 6 & 43452.7 & 63972.8 & & & & & $4^{n}$ Steel & $06-06-78$ & $04-01-84^{*}$ \\
\hline PSB 6A & 43436.0 & 63975.7 & 292.1-262.1 & 322.1 & 324.2 & 62.1 & $4^{n} \mathrm{PVC}$ & $03-21-84$ & \\
\hline PBB 7 & 43538.1 & 64300.9 & & & & & 4" Steel & $06-06-78$ & $04-01.84^{*}$ \\
\hline PSB 7A & 43553.3 & 64301.0 & $289.0-259.0$ & 329.0 & 330.7 & 71.7 & 4" PVC & $03-14-84$ & \\
\hline
\end{tabular}

\section{PSS WELL SERIES}

Description: Par Fond Sludge Land Application Site

Bldg. Number: :'61-5G

Map: Figure A-25

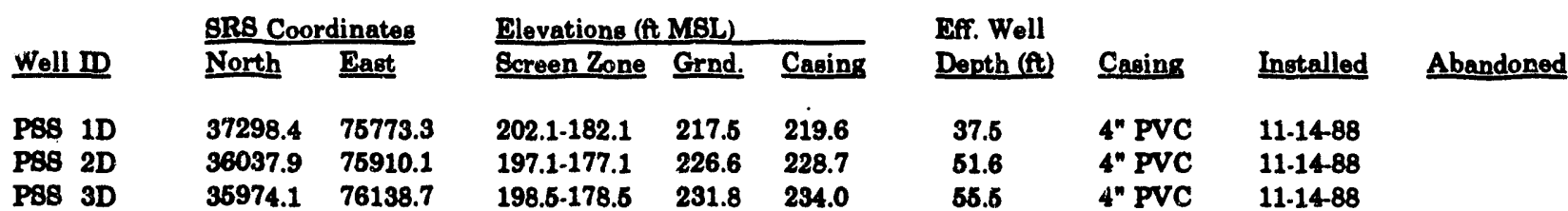

\section{PW WELL SERIES}

Description: Production Wells

Bldg. Number: Not applicable

Map: Figure A-1

\begin{tabular}{|c|c|c|c|c|c|c|c|c|c|}
\hline \multirow[b]{2}{*}{ Well ID } & \multicolumn{2}{|c|}{ SRS Coordinates } & \multicolumn{3}{|c|}{ Elevations (ft MBL) } & \multirow{2}{*}{$\begin{array}{l}\text { Eff. Well } \\
\text { Depth (t) }\end{array}$} & \multirow[b]{2}{*}{ Caging } & \multirow[b]{2}{*}{ Installed } & \multirow[b]{2}{*}{ Abandoned } \\
\hline & North & East & Bcreen Zone & Grad. & Casing & & & & \\
\hline $\begin{array}{ll}\text { PW } & \text { 2G } \\
\text { PW } & 8 \mathrm{G}\end{array}$ & $\begin{array}{l}76645 \\
50476.8\end{array}$ & $\begin{array}{l}17704 \\
89058.6\end{array}$ & & $\begin{array}{l}103.6 \\
268.9\end{array}$ & $\begin{array}{l}106.0 \\
271.4\end{array}$ & & "* & $\begin{array}{l}11-09-51 \\
06-27-52\end{array}$ & $\begin{array}{c}1977 \\
08-24-88\end{array}$ \\
\hline $\begin{array}{l}\text { PW 9G } \\
\text { PW 69G }\end{array}$ & $\begin{array}{l}106906.0 \\
106894.9\end{array}$ & $\begin{array}{l}72165.7 \\
72135.7\end{array}$ & $226.3-221.3$ & $\begin{array}{l}417.3 \\
269.7\end{array}$ & $\begin{array}{l}418.3 \\
272.2\end{array}$ & 197.0 & $\begin{array}{l}6^{n} \text { Bteel } \\
4^{n} \text { Iron }\end{array}$ & $\begin{array}{l}07-17-62 \\
09-01-65\end{array}$ & $\begin{array}{l}04-21-89 \\
05-16-89\end{array}$ \\
\hline
\end{tabular}

Notes: Although there is no abandonment report for $\mathrm{PW} \oplus 6 \mathrm{G}$, according to the Daily Activities Reports for this well, it was abandoned 6-15-89.

** PW 2G had an 8-in. steel casing inside an 18-in oteel casing. The screen length was $90 \mathrm{ft}$. 


\section{PW Wells}

Description: Unofficial wells in $\mathrm{Z}$ Area

Bldg. Number: Not applicable

Map: Figure A-16

\begin{tabular}{|c|c|c|c|c|c|c|c|c|c|}
\hline \multirow{2}{*}{\multicolumn{2}{|c|}{ Well ID }} & \multicolumn{2}{|c|}{ BRS Coordinates } & \multicolumn{2}{|l|}{ Elevations (ft MSL) } & \multirow{2}{*}{$\begin{array}{l}\text { Eff. Well } \\
\text { Depth (ft) }\end{array}$} & \multirow[b]{2}{*}{ Casing } & \multirow[b]{2}{*}{ Installed } & \multirow[b]{2}{*}{ Abandoned } \\
\hline & & North & East & Screen Zone Grnd. & Casing & & & & \\
\hline PW & 1 & 77900 & 67200 & & & & 2" PVC & & $04-22-87$ \\
\hline PW & 2 & 77880 & 67050 & & & & $2^{n}$ PVC & & $04-03-87$ \\
\hline PW & 3 & 77850 & 67000 & & & & $2^{n}$ PVC & & $04-03-87$ \\
\hline PW & 4 & 77830 & 66900 & & & & 2" PVC & & $04-03-87$ \\
\hline PW & 5 & 77810 & 66810 & & & & $2^{n}$ PVC & & $04-02-87$ \\
\hline PW & 6 & 77780 & 66710 & & & & $2^{n}$ PVC & & $04-02-87$ \\
\hline PW & 7 & 77990 & 66240 & & & & 2" PVC & & $04-22-87$ \\
\hline PW & 8 & 78100 & 67220 & & & & 2" PVC & & $04-22-87$ \\
\hline PW & 9 & 77810 & 67280 & & & & $2^{n} \mathrm{PVC}$ & & $04-03-87$ \\
\hline PW & 10 & 77740 & 67300 & & & & 2" PVC & & $04-03-87$ \\
\hline PW & 11 & 77870 & 67570 & & & & $2^{n}$ PVC & & $04-01.87$ \\
\hline FW & 12 & 76990 & 66320 & & & & 2" PVC & & $03-31-87$ \\
\hline PW & 13 & 77950 & 67390 & & & & $2^{n}$ PVC & & \\
\hline PW & 14 & 77970 & 67490 & & & & $2^{n}$ PVC & & $34-02-87$ \\
\hline PW & 15 & 77990 & 67590 & & & & 2" PVC & & $04-02-87$ \\
\hline PW & 16 & 77080 & 66360 & & & & $2^{*}$ PVC & & $03-31.87$ \\
\hline PW & 17 & 77170 & 66390 & & & & $\mathbf{2}^{n}$ PVC & & $03-31-87$ \\
\hline PW & 18 & 76890 & 66390 & & & & $2^{n}$ PVC & & $08-31-87$ \\
\hline PW & 19 & 76920 & 66190 & & & & $2^{*}$ PVC & & $04-02-87$ \\
\hline PW & 20 & 76920 & 66590 & & & & $2^{n}$ PVC & & $04-03-87$ \\
\hline PW & 21 & 76960 & 66210 & & & & $2^{N}$ PVC & & $03-31-87$ \\
\hline PW & 22 & 76800 & 66250 & & & & PVC & & $03-81-87$ \\
\hline
\end{tabular}

Notes: These PW Wells are not production wells. The identification of these wells comes from the abandonment sheets. The PW designation is unofficial.

\section{PZ WELL SERIES}

Description: Z-Area Piezometer Wells

Bldg. Number: Not applicable

Map: Figure A-16

\begin{tabular}{|c|c|c|c|c|c|c|c|c|}
\hline \multirow[b]{2}{*}{ Well ID } & \multicolumn{2}{|c|}{ BRS Coordinates } & \multicolumn{2}{|l|}{ Elevations (ft MSI) } & \multirow{2}{*}{$\begin{array}{l}\text { Eff. Well } \\
\text { Depth (ft) }\end{array}$} & \multirow[b]{2}{*}{ Casing } & \multirow[b]{2}{*}{ Ingtalled } & \multirow[b]{2}{*}{ Abandoned } \\
\hline & North & East & Bcreen Zone Grnd. & Casing & & & & \\
\hline PZ 101 & & & & & & $2^{n}$ PVC & & $01-10-87$ \\
\hline PZ 106 & 76327 & 66318 & & & & 2n PVC & & $09-30-87$ \\
\hline PZ 122 & 78645 & 67118 & & & & 2" PVC & & $10-02-87$ \\
\hline PZ 125 & 75207 & 68250 & & & & 2" PVC & & 05.29 .87 \\
\hline PZ 128 & & & & & & & & $07-09.86$ \\
\hline PZ 129 & 76680 & 67660 & & & & $2^{n}$ PVC & & $06-03-87$ \\
\hline $\mathrm{PZ} 202$ & 76107 & 67272 & & & & $2^{n}$ PVC & & $05-21-87$ \\
\hline PZ 210 & & & & & & 2" PVC & & $03-06-87$ \\
\hline PZ 216 & & & & & & 2n PVC & & $03-02-87$ \\
\hline PZ 218 & 77297 & 67251 & & & & $4^{n}$ PVC & & $06-08-87$ \\
\hline 2222 & 77585 & 66139 & & & & 2" PVC & & $01-10-87$ \\
\hline
\end{tabular}


RAB WELL SERIES (see RAC WELL SERIES)

RAC WELL SERIES

Description: R-Area Acid/Caustic Basin

Bldg. Number: $904-77 \mathrm{G}$

Map: Figure A-7

\begin{tabular}{|c|c|c|c|c|c|c|c|c|c|}
\hline \multirow[b]{2}{*}{ Well ID } & \multicolumn{2}{|c|}{ SRs Coordinates } & \multicolumn{3}{|c|}{ Elevations (A MSL) } & \multirow{2}{*}{$\begin{array}{l}\text { Bff. Well } \\
\text { Depth (it) }\end{array}$} & \multirow[b]{2}{*}{ Casing } & \multirow[b]{2}{*}{ Installed } & \multirow[b]{2}{*}{ Abandoned } \\
\hline & North & East & Screen Zone & Grnd. & Casing & & & & \\
\hline 1 & 65107.3 & 74570.7 & $277.3-247.3$ & 282.3 & 283.6 & 36.3 & 4" PVC & $10-21-83$ & \\
\hline RAC 2 & 55026.3 & 74655.5 & $273.4-243.4$ & 278.4 & 280.4 & $\mathbf{3 7 . 0}$ & 4" PVC & $10-24-83$ & \\
\hline RAC 3 & 66016.3 & 74667.5 & $272.3-242.3$ & 277.3 & 279.3 & $\mathbf{3 7 . 0}$ & 4" PVC & $10-25-83$ & \\
\hline RAC 4 & 54984.0 & 74588.8 & 268.2-238.2 & 277.2 & 279.0 & 40.8 & $4^{n} \mathrm{PVC}$ & $07-02-84$ & \\
\hline
\end{tabular}

Notes: The RAC Series has been designated the RAB Series in the part.

\section{RCP WELL SERIES}

Description: R-Area Coal Pile Study

Bldg. Number: Not applicable

Map: Figure A.7

\begin{tabular}{|c|c|c|c|c|c|}
\hline \multirow[b]{2}{*}{ Well ID } & \multicolumn{2}{|c|}{ SR8 Coordinates } & \multicolumn{2}{|c|}{ Plevations (A MSL) } & \multirow[b]{2}{*}{ Coning } \\
\hline & North & Bant & Ecroen Zone & Grnd. & \\
\hline $\begin{array}{ll}\text { RCP } & \text { AA } \\
\text { RCP } & \text { ID }\end{array}$ & $\begin{array}{l}66968.1 \\
66967.9\end{array}$ & $\begin{array}{l}74238.3 \\
74223.6\end{array}$ & $\begin{array}{r}66.846 .8 \\
281.3-261.3\end{array}$ & $\begin{array}{l}294.8 \\
294.8\end{array}$ & $\begin{array}{l}296.9 \\
296.8\end{array}$ \\
\hline
\end{tabular}

Ef. Well

Depth(t) Casing Intalled Abandoned

$200.1 \quad 4^{\prime \prime}$ PVC

35.6 4" PVC
08-17-90

07-18-90

\section{RDB WELL SERIES}

Description: R-Area Disassembly Basin

Bldg. Number: 105-R

Map: Figure A-7

\begin{tabular}{|c|c|c|c|c|c|c|c|c|c|}
\hline \multirow[b]{2}{*}{ Well ID } & \multicolumn{2}{|c|}{ ERs Coordinatos } & \multicolumn{3}{|c|}{ Hevations (A MSL) } & \multirow{2}{*}{$\begin{array}{l}\text { Efi. Woll } \\
\text { Depth (ft) }\end{array}$} & \multirow[b]{2}{*}{ Caning } & \multirow[b]{2}{*}{ Installod } & \multirow[b]{2}{*}{ Abandoned } \\
\hline & North & Esat & Eareen Zone & Grad. & Caning & & & & \\
\hline $\begin{array}{ll}\text { RDB } & \text { ID } \\
\text { RDB } & \text { 2D } \\
\text { RDB } & \text { 3D }\end{array}$ & $\begin{array}{l}67097.3 \\
66879.8 \\
66881.9\end{array}$ & $\begin{array}{l}74844.5 \\
74782.2 \\
74899.0\end{array}$ & $\begin{array}{l}285.6-265.6 \\
285.7-265.7 \\
285.8-265.8\end{array}$ & $\begin{array}{l}290.5 \\
290.7 \\
290.8\end{array}$ & $\begin{array}{l}292.4 \\
292.6 \\
292.7\end{array}$ & $\begin{array}{l}26.9 \\
26.9 \\
26.9\end{array}$ & $\begin{array}{l}\text { 4" PVC }^{\prime \prime} \text { PVC } \\
\text { 4" PVC }^{\prime \prime} \text { PVC }\end{array}$ & $\begin{array}{l}02-27-90 \\
02-26-90 \\
02-27.90\end{array}$ & \\
\hline
\end{tabular}




\section{RRP WELL SERIES}

Description: R-Area Burning/Rubble Pits

Bldg. Number: 131-R, -1R

Map: Figure A-7

\begin{tabular}{|c|c|c|c|c|c|c|c|c|c|}
\hline \multirow[b]{2}{*}{ Well ID } & \multicolumn{2}{|c|}{ SRS Coordinates } & \multicolumn{3}{|c|}{ Elevations (ft MSL) } & \multirow{2}{*}{$\begin{array}{l}\text { Bff. Well } \\
\text { Depth (ft) }\end{array}$} & \multirow[b]{2}{*}{ Casing } & \multirow[b]{2}{*}{ Installed } & \multirow[b]{2}{*}{ Abandoned } \\
\hline & North & East & Bcreen Zone & Grnd. & Casing & & & & \\
\hline RRP 1 & 64663.6 & 75634.6 & $272.4-242.4$ & 282.4 & 284.4 & 42.0 & $4^{n}$ PVC & $10-20-83$ & \\
\hline RRP 2 & 54468.3 & 75829.8 & $272.6-242.5$ & 282.6 & 284.6 & 42.0 & 4" PVC & $10-19.83$ & \\
\hline RRP 3 & 54303.0 & 75853.0 & 268.1-238.1 & 278.1 & 280.1 & 42.0 & $4^{n}$ PVC & $10-20-83$ & \\
\hline RRP 4 & 54294.5 & 75723.3 & 268.3-238.3 & 278.3 & 280.2 & 41.9 & $4^{\prime \prime}$ PVC & $07-02-84$ & \\
\hline
\end{tabular}

\section{RSA WELL SERIES}

Description: Series A, R-Area Reactor Seepage Basins

Bldg. Number: 904-60G

Map: Figure A-7

\begin{tabular}{|c|c|c|c|c|c|c|c|c|c|}
\hline & SR8 Coo & dinates & Flevations (f & MSL & & Bff. Well & & & \\
\hline Well ID & North & East & Ecreen Zone & Grnd. & Casing & Depth (ft) & Casing & Installed & Abandoned \\
\hline RSA 1 & 57872.3 & 74820.7 & & 307.2 & 310.4 & & Steel & $01-14-58$ & $04-19.61$ \\
\hline RSA 2 & 57995.6 & 74828.4 & 274.7. & 308.7 & 313.0 & & 3" Btoel & $01-16-68$ & $04-19-61$ \\
\hline RSA 3 & 68075.6 & 74895.6 & 274.6- & 307.6 & 310.4 & & $3^{n}$ Steel & $01-17.68$ & $04-19-61$ \\
\hline RSA 4 & 68312.6 & 74843.6 & 283.9- & 304.9 & 307.4 & & 3" Steel & $01-27-58$ & $04-19-61$ \\
\hline $\begin{array}{l}\text { RSA 4A } \\
\text { RSA }\end{array}$ & 58360.6 & 74799.0 & 280.2 & 301.7 & 303.9 & & $\begin{array}{l}3^{n} \text { Stool } \\
\text { Stoel }\end{array}$ & $01-31-58$ & $\begin{array}{l}04-19-61 \\
04-19-61\end{array}$ \\
\hline $\begin{array}{ll}\text { RSA } & 5 \\
\text { RSA } & 5 A \\
\text { RSA } & \text { 5B }\end{array}$ & 58421.6 & 78854.0 & 281.1- & 302.8 & 307.1 & & $\begin{array}{l}\text { 3" Bteel } \\
\text { Streel } \\
\text { Steel }\end{array}$ & $01-31-68$ & $\begin{array}{l}04-19-61 \\
04-19-61\end{array}$ \\
\hline RSA 6 & 68159.0 & 76000.0 & 270.0 & 309.0 & 312.3 & & 3n Btoel & $01-30-68$ & $04=15-61$ \\
\hline RSA 7 & 58065.7 & 75195.9 & 276.3 & 311.8 & 314.7 & & 3n Steol & $04-16-68$ & \\
\hline RSA 7 & 68065.7 & 75196.9 & 289.5-269.6 & $\mathbf{3 1 0 . 6}$ & 312.4 & 42.8 & 4" PVC & $11-26-84$ & \\
\hline RSA 8 & 58068.3 & 75402.3 & & 311.2 & 314.3 & & 3" Btoel & $04-16-68$ & \\
\hline RSA 8 & 58068.3 & 75402.3 & 286.5-265.6 & 310.2 & 312.3 & 46.7 & 4" PVC & $11-21-84$ & \\
\hline RSA 9 & 58129.8 & 75616.2 & 274.3 & 309.3 & 312.1 & & 3" Stoel & $04-16-58$ & \\
\hline RSA 9 & 68123.8 & 75616.2 & $284.6-264.6$ & 309.7 & 311.7 & 47.1 & $4^{n}$ PVC & 11.21 .84 & \\
\hline RSA 10 & 58172.8 & 75389.3 & 277.8 & 312.8 & 316.4 & & $3^{n}$ Steel & $04-17-68$ & \\
\hline RSA 10 & 58172.8 & 75389.3 & 288.7-268.8 & 309.7 & 311.3 & 42.5 & 4" PVC & $11-26-84$ & \\
\hline RSA 11 & 58494.6 & 76538.0 & & 302.4 & 305.5 & & Steel & $04-01-58 *$ & $04-19-61$ \\
\hline RSA 12 & 58573.6 & 75173.6 & 268.9 & 302.9 & 305.9 & & 3" Steel & $04-17-58$ & $04-19-61$ \\
\hline RSA 13 & 68500.0 & 74958.0 & 269.6 & 304.6 & 307.3 & & $3^{n}$ Bteel & $04-18-68$ & $04-19-61$ \\
\hline
\end{tabular}

Notes: As the result of a monitoring program review, sampling of 12 Series A wells was stopped in 1961 (Ashley and Zeigler, 1978). RSA 6 was still being read as late as 5-17.63. The abandonment dates listed are the last date the wells were sampled.

The 3 in. oteel casings in RAM 7, 8, 9, and 10 were replaced with 4 in. PVC casings in November 1984 when these welle were redrilled. 


\section{RSB WELL SERIES}

Description: Series B, R-Area Reactor Seepage Basins Bldg. Number: 904-103G and an abandoned sewer line Map: Figure A-7

\begin{tabular}{|c|c|c|c|c|c|c|c|c|c|}
\hline \multirow[b]{2}{*}{ Well ID } & \multicolumn{2}{|c|}{ gRS Coordinates } & \multicolumn{3}{|c|}{ Glevations (it MSL) } & \multirow{2}{*}{$\begin{array}{l}\text { Eff. Well } \\
\text { Depth (ft) }\end{array}$} & \multirow[b]{2}{*}{ Casing } & \multirow[b]{2}{*}{ Ingtalled } & \multirow[b]{2}{*}{ Abandoned } \\
\hline & North & East & Scroen Zone & Grnd. & Casing & & & & \\
\hline RSB 1 & 57746.4 & 74507.6 & & & 502.6 & & Stoel & $01-16-58$ & $04-19-61$ \\
\hline RSB 2 & 67965.6 & 74734.3 & 274.1. & & 805.6 & & $1.6^{\prime \prime}$ Steel & $01-15.68$ & $04-19-61$ \\
\hline RSB 3 & 58411.4 & 74724.4 & & & 302.9 & & Stoel & $01-16-58$ & $04-19.61$ \\
\hline RSB 4 & 68640.9 & 74992.2 & 264.6. & & 303.0 & & $1.5^{n}$ Steel & $01-23-68$ & $04-19-61$ \\
\hline RSB 4 & 58644.0 & 74926.0 & & & 901.6 & & Steel & $03-01.58^{*}$ & $04-19-61$ \\
\hline RSB 41 & & & & & & & Stoel & & $04-19-61$ \\
\hline RSB 6 & 68596.0 & 76313.3 & 267.0. & & 305.6 & & $1.6^{\circ}$ Bteel & 01.2258 & $04-19-61$ \\
\hline RSB 6 & 58249.2 & 75102.0 & 272.1. & & 309.5 & & $1.5^{n}$ Stoel & $01-23-68$ & $04-19-61$ \\
\hline RSB 7 & 57692.8 & 75044.3 & & & 308.1 & & Steel & $01.22-68$ & \\
\hline RSB 7 & 67692.8 & 76044.8 & 292.6-272.7 & 307.2 & 309.0 & 36.3 & 4" PVC & $11-20-84$ & \\
\hline RSB 8 & 67612.9 & 75178.2 & 294.3-274.3 & 304.3 & 305.8 & 31.5 & $4^{n}$ Bteel & $09-26-76$ & \\
\hline RBB 9 & 57527.9 & 75378.3 & 264.9 & 303.9 & 305.6 & & $4^{n}$ Oteel & $02-25-77$ & \\
\hline
\end{tabular}

Notes: As the result of a monitoring program review, sampling of eight Series B wells was discontinued in 1961 (Ashley and Zeigler, 1978). The abandonment date lieted is the last date the wells were eampled.

The steel casing in PSB 7 was replaced with a 4 in PVC casing in November 1984 when the well was redrilled.

\section{RSC WELL SERIES}

Description: Series C, R-Area Reactor Seepage Basins Bldg. Number: 904-103G, -104G, -57G, -58G, -59G, -60G Map: Figure A-7

\begin{tabular}{|c|c|c|c|c|c|c|c|c|c|}
\hline & BRs Cor & dinater & Flevations (f & MSL) & & EF. Well & & & \\
\hline Well $\mathrm{I}$ & North & East & Screen Zone & Grod. & Casing & Depth (A) & Casing & Intalled & Abandoned \\
\hline $\begin{array}{ll}R S C C & 1 \\
R S C & 2\end{array}$ & 58111.6 & 74389.4 & 266.9. & 302.9 & 306.1 & & $1.5^{\circ}$ Stoel & 01-16-68 & \\
\hline RSC 2 & 58543.0 & 74378.6 & $281.9-261.9$ & 299.9 & 302.0 & 40.1 & $4^{n}$ 8teel & $10-22-76$ & \\
\hline RSC $\mathrm{s}$ & 58724.7 & 74699.7 & & & 302.8 & & 1.5" Bteel & $01-20-68$ & \\
\hline RsC 3 & 68724.7 & 74699.7 & 278.6-258.6 & 299.6 & 301.3 & 42.7 & $4^{\prime \prime}$ Stoel & $10-27-76$ & \\
\hline RSC 4 & 68900.3 & 76097.8 & & & 301.3 & & Stoel & $01-20-68$ & \\
\hline $\begin{array}{ll}\mathrm{RSC} & 4 \\
\mathrm{RSC} & 5\end{array}$ & 68900.3 & 75097.8 & 288.6-288.6 & 298.6 & 300.3 & 31.7 & 4" Bteel & 10-22-78 & \\
\hline RsC 5 & 58901.2 & 75484.9 & 278.3-258.3 & 302.3 & 30.9 & 46.6 & 1.0" Stoel & $\begin{array}{l}01-23-68 \\
10-23-76\end{array}$ & \\
\hline RsC 6 & 68607.1 & 75686.1 & & & 303.9 & & Stoel & $01-22-68$ & \\
\hline RSC 6 & 58607.1 & 75686.1 & 287.7-267.7 & 300.7 & 304.1 & 36.4 & $4^{\infty}$ Steel & $10-21-76$ & \\
\hline RBC 7 & 68200.1 & 75886.2 & & & 309.0 & & 1.5" Stool & $01-23-68$ & \\
\hline RsC 7 & 68200.1 & 76686.2 & $283.4-263.4$ & 306.4 & 307.8 & 44.4 & 4" Bteel & $10-21-76$ & \\
\hline R8C 8 & 67818.1 & 75684.0 & & & $\$ 10.3$ & & Steel & $01-22-68$ & \\
\hline RSC 8 & 67818.1 & 76684.0 & & 307.3 & 309.8 & & $4^{*}$ Stroel & $10-21-76$ & \\
\hline RsC 9 & 69241.2 & 74565.3 & & & 303.2 & & 3" Btoel & $01-21-68$ & \\
\hline RsC 9 & 69241.2 & 74566.3 & 271.6-251.6 & 299.6 & 301.8 & 60.2 & $4^{n}$ Bteel & 11.0476 & \\
\hline RSC 10 & 69642.7 & 75470.0 & & 296.4 & & & $3^{n}$ Stoel & $04-14-58$ & \\
\hline RSC 10 & 59542.7 & 75470.0 & 275.5-255.5 & 293.6 & 297.4 & 41.9 & $4^{n}$ Steel & $10-22-76$ & \\
\hline
\end{tabular}

(eeries continued) 


\section{RSC WELL SERIES (cont.)}

Notes: Four-inch galvanized steel casings replaced the original steel casings in the same boreholes in RsC 2, 3, 4, 5, 6, 7, 8, and 10 in October 1976 when these wells were reworked.

The Drilling Request for R8C 9 indicates that the old well could not be pulled; therefore, the casing was uncoupled 15 ft below ground level, the hole was filled with wet cement, and a new well was drilled.

\section{RSD WELL SERIES}

Description: Series D, between R-Area Reactor Seepage Basins and R-Area Disassembly Basin Bldg. Number: 904-103G, 105-R

Map: Figure A-7

\begin{tabular}{|c|c|c|c|c|c|c|c|c|c|}
\hline \multirow[b]{2}{*}{ Well ID } & \multicolumn{2}{|c|}{ SRS Coordinates } & \multicolumn{3}{|c|}{ Elevations (ft MSL) } & \multirow{2}{*}{$\begin{array}{l}\text { Eff. Well } \\
\text { Depth (ft) }\end{array}$} & \multirow[b]{2}{*}{ Casing } & \multirow[b]{2}{*}{ Installed } & \multirow[b]{2}{*}{ Abandoned } \\
\hline & North & East & Screen Zone & Grnd. & Casing & & & & \\
\hline RSD 1 & 57440.8 & 75035.1 & 266.6 & & 301.0 & & $3^{n}$ Steel & $01-27-58$ & \\
\hline RSD 1 & 67440.8 & 75035.1 & 287.7-267.9 & 298.7 & 300.6 & 32.6 & $4^{n}$ PVC & $11-26-84$ & \\
\hline RED 2 & & & 268.5. & 299.0 & 301.2 & & $3^{n}$ Steel & $01-24-68$ & $07.90-64$ \\
\hline RSD $2 A$ & 57448.5 & 74811.6 & & 298.4 & 301.2 & & Steel & $07-30-64$ & \\
\hline RSD 2B & 57468.9 & 74810.7 & & 299.4 & 303.6 & & Steel & $07-30-64$ & \\
\hline RSD 2C & 57479.3 & 74810.6 & & 299.7 & 301.7 & & Steel & $04-17-64$ & \\
\hline RSD 3 & 57451.6 & 74702.3 & 262.7 & & & & $3^{n}$ Steel & $01-24-68$ & \\
\hline RED 3 & 57451.6 & 74702.3 & 289.1-269.3 & 298.9 & 300.8 & 31.5 & 4" PVC & $11.19-84$ & \\
\hline RSD 4 & 57441.4 & 76154.6 & $290.6-270.6$ & 299.6 & 301.6 & 91.0 & 4" Btoel & $09-24-76$ & \\
\hline RSD 5 & 67499.9 & 75207.0 & $289.6-269.6$ & 299.6 & 501.7 & 32.1 & $4^{\prime \prime}$ Steel & $10-06-76$ & \\
\hline RSD 6 & 67441.3 & 75256.6 & $290.1-270.1$ & 300.1 & 302.1 & $\$ 2.0$ & 4" Bteel & $10-06-76$ & \\
\hline RSD 7 & 67994.3 & 75178.4 & $287.9-267.3$ & 291.3 & 293.4 & 26.1 & 4" Bteel & $02-28.77$ & \\
\hline RSD 8 & 67994.0 & 75229.6 & 287.3-267.3 & 291.3 & 293.0 & 26.7 & $4^{n}$ Bteel & 02-28-77 & \\
\hline RSD 9 & 57245.6 & 75186.9 & 271.7-251.7 & 290.7 & 292.6 & 40.9 & $4^{n}$ Bteel & 02-25-77 & \\
\hline RSD 10 & 57246.6 & 75235.8 & & 290.5 & 292.5 & & Stoel & $10-01-77^{*}$ & \\
\hline RSD 11 & 67249.1 & 76285.6 & & 290.4 & 292.3 & & Steol & $10-01.77^{*}$ & \\
\hline
\end{tabular}

Notes: PVC casing replaced stoel casing in RBD 1 and $\mathrm{BSD} 3$.

\section{RSE WELL SERIES}

Description: Series E, R-Area Reactor Seepage Basins Bldg. Number: 904-103G, -57G, -58G

Map: Figure A-7

\begin{tabular}{|c|c|c|c|c|c|c|c|c|c|}
\hline \multirow[b]{2}{*}{ Well ID } & \multicolumn{2}{|c|}{ SR8 Coordinates } & \multicolumn{3}{|c|}{ Elevations (ft MSL) } & \multirow{2}{*}{$\begin{array}{l}\text { Eff. Well } \\
\text { Depth (ft) }\end{array}$} & \multirow[b]{2}{*}{ Casing } & \multirow[b]{2}{*}{ Installed } & \multirow[b]{2}{*}{ Abandoned } \\
\hline & North & $\overline{\text { East }}$ & Bcreen Zone & Grnd. & Casing & & & & \\
\hline RSE 1 & & & & & & & Steel & $07.01-63^{*}$ & \\
\hline IRSE $1 A$ & 57734.5 & 74712.7 & 272.4-262.4 & 302.4 & 304.4 & 42.0 & Steel & $04-02-63$ & \\
\hline RSE IA & 57734.5 & 74712.7 & $294.8-274.8$ & 302.3 & 304.2 & 29.4 & $4^{\prime \prime} \mathrm{PVC}$ & $1.1-13-84$ & \\
\hline R8E 1B & 57731.4 & 74698.1 & & 301.2 & 303.7 & & Steel & $04-17-64$ & \\
\hline R8E 1B & 57731.4 & 74698.1 & 295.7-275.7 & 301.7 & 303.3 & 27.6 & 4" PVC & $11-12-84$ & \\
\hline RSE 1C & 57730.8 & 74684.1 & & 301.1 & 303.6 & & Stool & $04-15-64$ & \\
\hline REE $1 \mathrm{C}$ & 57730.8 & 74684.1 & 288.5-268.6 & 301.5 & 303.3 & 34.8 & 4" PVC & $11-14-84$ & \\
\hline RSE 2 & 67594.9 & 74743.6 & 268.8-258.8 & 300.8 & 302.8 & 44.0 & Steol & $0402-63$ & \\
\hline
\end{tabular}

(series continued) 
RSE WELL SERIES (cont.)

\begin{tabular}{|c|c|c|c|c|c|c|c|c|c|}
\hline \multirow{2}{*}{ Well ID } & \multicolumn{2}{|c|}{ SR8 Coordinates } & \multicolumn{3}{|c|}{ Flevations (ft MSL) } & \multirow{2}{*}{$\begin{array}{l}\text { Bff. Well } \\
\text { Depth (tt) }\end{array}$} & \multirow[b]{2}{*}{ Casing } & \multirow[b]{2}{*}{ Installed } & \multirow[b]{2}{*}{ Abandoned } \\
\hline & North & Fast & Screen Zone & Grnd. & Casing & & & & \\
\hline RBE 2 & 57594.9 & 74743.8 & 289.5-269.7 & 300.7 & 502.6 & 32.8 & 4" PVC & $11.09-84$ & \\
\hline RSE 3 & 57444 & 74992 & 269.4-253.4 & 299.4 & 301.4 & 48.0 & Steel & $04-02-63$ & \\
\hline R8E 3 & 67445.8 & 74931.2 & 288.0-268.2 & 299.2 & 301.0 & 32.8 & $4^{\prime \prime}$ PVC & $11-13-84$ & \\
\hline R8E 4 & & & & & & & Steel & $07-01.63$ & \\
\hline R8E 4 & 57528.4 & 75101.1 & $270.6-260.6$ & 302.6 & 304.6 & 44.0 & Steel & $04-05-63$ & \\
\hline RSE 41 & 57581.0 & 75096.2 & & 302.8 & 305.9 & & Stoel & $07-01-64 *$ & \\
\hline RSE 4 & 57539.6 & 76092.6 & & 303.1 & 304.7 & & Bteel & $07-01-64^{*}$ & \\
\hline RSE 6 & 57588.9 & 74969.6 & & 304.0 & 306.0 & & Steel & $04-03-63$ & \\
\hline RSE 6 & 57592.1 & 74815.1 & & 302.6 & 304.6 & & Bteel & $04-03-63$ & \\
\hline RSE 7 & 58481.5 & 74783.7 & $264.0-254.0$ & 301.0 & 303.0 & 49.0 & Stoel & $04-05-63$ & \\
\hline RSE 7 & 58481.6 & 74783.7 & 286.3-266.6 & 300.9 & 302.4 & 35.9 & $4^{n}$ PVC & $11-15-84$ & \\
\hline RSE 8 & 58538.8 & 74869.4 & 263.1-253.1 & 300.1 & 302.1 & 49.0 & Btoel & $04-05-63$ & \\
\hline RSE 8 & 58538.8 & 74869.4 & 291.0.271.2 & 300.2 & 302.2 & 31.0 & $4^{n}$ proc & $11.14-84$ & \\
\hline R8E 9 & 58463.3 & 74971.1 & 266.8-256.8 & 305.8 & 307.8 & 61.0 & Stoel & $04-05-63$ & \\
\hline R8E 9 & 58463.3 & 74971.1 & 286.7-266.7 & 304.2 & 308.0 & 39.3 & 4" PVC & $11-15-84$ & \\
\hline RBE 10 & 58420.7 & 74848.3 & & 305.3 & $\$ 07.8$ & & Steel & $05-18-63$ & \\
\hline RSE 10 & 68420.7 & 74848.3 & $290.6-270.7$ & 303.2 & 804.7 & 34.0 & $4^{n}$ PVC & $11.15-84$ & \\
\hline RSE 11 & 58357.6 & 74787.7 & & 302.1 & 308.8 & & Steel & $05.18-63$ & \\
\hline RSE 12 & 58318.2 & 74842.3 & & 304.1 & 905.8 & & Steel & $05-18-63$ & \\
\hline RSE 13 & 67507.6 & 75146.7 & & 300.9 & 301.2 & & Btoel & 04-15-63 & \\
\hline RSE 14 & & & & 302.0 & 304.0 & & Steul & $04-17.65$ & \\
\hline RSE 15 & & & & 302.2 & 903.3 & & Stoel & $04-17-64$ & \\
\hline RSE 16 & & & & 302.7 & 304.6 & & Steel & $04-17.64$ & \\
\hline RSE 17 & & & & 303.1 & 305.2 & & Steel & & \\
\hline REE 18 & 58247.2 & 74859.6 & 288.1-268.1 & $\$ 05.1$ & 307.1 & 39.0 & $4^{\prime \prime}$ Btoel & 10.07 .76 & \\
\hline RSE 19 & 58318.4 & 74791.2 & $282.6-262.5$ & 902.5 & 304.8 & 42.3 & $4^{\prime \prime}$ Btoel & $10-07-76$ & \\
\hline REE 24 & 67370.4 & $7 e \times 8.9$ & $267.6-237.6$ & 291.6 & 294.1 & 66.5 & $4^{\prime \prime}$ PVC & $01-08-87$ & \\
\hline RSE 25 & 56824.5 & 74544.6 & 257.6-237.5 & 291.6 & 298.9 & 66.4 & $4^{\prime \prime}$ PVC & $01-07-87$ & \\
\hline
\end{tabular}

Notes: Ashley and Zoigler (1978) ataten that the firat 12 Page welle were inctallod in 1969. Based on thin information, Bes: 1 is given an inetalled date of 7-01-63. The field $\log$ for PST 15 indicates this well was inetalled in 1963.

PVC casing reploced etcel casing in Rest 1A, 1B, 1C, and 2.

\section{RSF WELL SERIES}

Description: Series F, R-Area Reactor Seepage Basins

Bldg. Number: 904-103G, -57G, -58G

Map: Figure A-7

\begin{tabular}{|c|c|c|c|c|c|c|c|c|c|}
\hline \multirow[b]{2}{*}{ Well ID } & \multicolumn{2}{|c|}{ 8RS Coordinates } & \multicolumn{3}{|c|}{ Elevations (ft MSL) } & \multirow{2}{*}{$\begin{array}{l}\text { Defr. Well } \\
\text { Depth (A) }\end{array}$} & \multirow[b]{2}{*}{ Casing } & \multirow[b]{2}{*}{ Installed } & \multirow[b]{2}{*}{ Abandonod } \\
\hline & North & Bast & Screen Zone & Grnd. & Casing & & & & \\
\hline & 68505.3 & 74869.4 & 238.8-228.8 & 300.8 & 303.1 & 74.3 & $4^{\circ}$ PVC & $02-02-87$ & \\
\hline RBP 2 & 57670.4 & 74628.6 & $235.3-224.8$ & 300.3 & 302.8 & 78.0 & 4" PVC & $02-16-87$ & \\
\hline RBF 3 & 57621.4 & 75206.7 & $239.8-229.8$ & 304.8 & 307.1 & 77.3 & $4^{n}$ PVC & $02-24-87$ & \\
\hline
\end{tabular}




\section{RSS WELL SERIES}

Description: S-Area Defense Waste Processing Facility (DWPF)

Bldg. Number: Not applicable

Map: Figure A-16

\begin{tabular}{|c|c|c|c|c|c|c|c|c|c|}
\hline \multirow[b]{2}{*}{ Well ID } & \multicolumn{2}{|c|}{ SRs Coordinates } & \multicolumn{3}{|c|}{ Elevations (ft MSL) } & \multirow{2}{*}{$\begin{array}{l}\text { Eff. Well } \\
\text { Depth (ft) }\end{array}$} & \multirow[b]{2}{*}{ Casing } & \multirow[b]{2}{*}{ Ingtalled } & \multirow[b]{2}{*}{ Abandonor } \\
\hline & North & East & Boreen Zone & Grnd. & Casing & & & & \\
\hline Rs8 1 & 74100 & 65100 & 218.2-215.2 & 292.2 & 298.8 & 78.6 & 1" Steel & $01-22-75$ & \\
\hline RSS 2 & 74100 & 65900 & 200.2-197.2 & 274.2 & 277.2 & 80.0 & 1" Steel & $01-23-75$ & $02-09-88$ \\
\hline R8S 3 & 74800 & 64000 & $188.7-185.7$ & 262.7 & 264.5 & 78.8 & 1" Bteel & $01-24-75$ & $02-12-88$ \\
\hline RSS 4 & 74800 & 64800 & $213.6-210.6$ & 287.6 & 289.4 & 78.8 & 1" Bteel & $01-21-75$ & $02-11-88$ \\
\hline R8S 5 & 74800 & 65900 & 216.9-213.9 & 290.9 & 293.0 & 79.1 & 1" Steel & 01.20 .75 & $01-14-89$ \\
\hline
\end{tabular}

Notes: The BSS Beries has been designated the RSBP Series in the past.

RSSF WELL SERIES (see RSS WELL SERIES)

\section{RSW WELL SERIES}

Description: Series DW, R-Area Reactor Seepage Basins Dry Monitoring Wells Bldg. Number: 904-103G, -104G, -57G, -58G, -59G

Map: Figure A-7

\begin{tabular}{|c|c|c|c|c|c|}
\hline \multirow[b]{2}{*}{ Well ID } & \multicolumn{2}{|c|}{ SRS Coordinates } & \multicolumn{2}{|c|}{ Elevations (ft MgL) } & \multirow[b]{2}{*}{ Casin: } \\
\hline & North & Fagt & Bcreen Zone & Grnd. & \\
\hline RSW 1 & 57629.5 & 74872.7 & & 302.1 & 304.6 \\
\hline RBW 2 & $\mathbf{5 7 6 2 9 . 2}$ & 74846.2 & & 302.3 & 304.8 \\
\hline R8W 3 & 57779.8 & 74697.2 & & 300.7 & 309.2 \\
\hline RSW 4 & 57778.7 & 74690.6 & & 300.5 & $\mathbf{3 0 9 . 0}$ \\
\hline RSW 5 & 57909.3 & 74866.7 & & 305.6 & 908.1 \\
\hline RSW 6 & 57441.7 & 75256.5 & & 306.0 & 308.5 \\
\hline RSW 7 & 68189.8 & 74886.0 & & 305.4 & 307.9 \\
\hline RSW 8 & 58190.4 & 74886.0 & & 304.4 & 306.9 \\
\hline RSW 9 & 58458.8 & 75026.1 & & 303.7 & 308.2 \\
\hline RSW 20 & 58467.0 & 75011.6 & & 303.3 & 305.8 \\
\hline RSW 1: & 58468.1 & 75431.7 & & 305.9 & 308.4 \\
\hline RSW 12 & 58505.3 & 76429.9 & & 305.6 & 308.1 \\
\hline
\end{tabular}

Eff. Well

Depth (ft) Casing Installed Abandoned

2" Iron $\quad 10105.76$

$2^{n}$ Iron 10.05 .76

2 "Iron $\quad 10-05.76$

2 Iron $10-05-76$

2" Iron $10-06-76$

$2^{*}$ Iron $\quad 10-05-78$

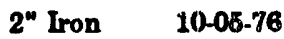

2" Iron 10.08.76

$2^{\text {N Iron } 10.05 \% \text {; }}$

2" Iron 10.05. 7

2 Iron 10-05.78

2" Iron 10-06. 36

Notes: The BSW wells are not groundwater monitoring wells. 


\section{RWM WELL SERIES}

Description: M-Area Recovery Wells

Bldg. Number: Not applicable

Map: Figures A-17 and A-18

\begin{tabular}{|c|c|c|c|c|c|c|c|c|c|}
\hline & 8RS Coord & linates & Plevations ( $\mathrm{t}$ & MSL) & & Eff. Well & & & \\
\hline Well ID & North & East & Screen Zone & Grnd. & Casing & Depth (ft) & Casing & Installed & Abandoned \\
\hline $\begin{array}{l}\text { RWM } 1^{2} \\
\text { RWM } 2\end{array}$ & $\begin{array}{l}102599.1 \\
104434.1\end{array}$ & $\begin{array}{l}48575.1 \\
49205.6\end{array}$ & $\begin{array}{l}232.3-172.3 \\
208.3-198.3 \\
188.3-178.3 \\
168.3-158.3 \\
148.9-138.3\end{array}$ & $\begin{array}{l}362.8 \\
368.3\end{array}$ & $\begin{array}{l}364.7 \\
371.3\end{array}$ & $\begin{array}{l}192.4 \\
293.0\end{array}$ & $8^{n} \mathrm{Cs}$ & $\begin{array}{l}05-07-85 \\
05-03-85\end{array}$ & \\
\hline RWM 3 & 104730.2 & 49680.0 & $\begin{array}{l}214.0-204.0 \\
194.0-184.0 \\
174.0-164.0 \\
154.0-144.0\end{array}$ & $\mathbf{3 7 4 . 0}$ & 377.0 & 233.0 & $8^{n} \mathrm{Cs}$ & $06-06-85$ & \\
\hline RWM 4 & 103719.3 & 48948.2 & $\begin{array}{l}211.9-201.6 \\
191.3-181.0 \\
170.7-160.4 \\
139.9-129.5\end{array}$ & 963.5 & 366.5 & 237.0 & $8^{n} \mathrm{CB}$ & $02-22-85$ & \\
\hline RWM 6 & 103502.2 & 49628.0 & $\begin{array}{l}216.8-206.4 \\
196.1-185.7 \\
166.1-154.7 \\
144.9-139.9\end{array}$ & 363.9 & $\$ 66.9$ & 293.0 & $8^{n} \mathrm{Cs}$ & $02-27-86$ & \\
\hline RWM 6 & 102001.5 & 50107.4 & $\begin{array}{l}218.7-208.4 \\
187.8-177.4 \\
167.1-166.8 \\
151.6-141.1\end{array}$ & 346.1 & 349.1 & 208.0 & $8^{n} \mathrm{Cs}$ & $05-03-85$ & \\
\hline RWM 7 & 101904.6 & 49449.6 & $\begin{array}{l}216.3-206.0 \\
196.7-185.3 \\
175.0-164.7 \\
154.2-144.0\end{array}$ & 346.0 & $\mathbf{3 4 9 . 0}$ & & $8^{n} \mathrm{Cs}$ & $02-25-85$ & \\
\hline RWM 8 & 101948.2 & 47353.3 & $\begin{array}{l}197.2-186.9 \\
176.6-166.2 \\
140.9-130.0 \\
119.7-109.3\end{array}$ & 345.3 & $\$ 48.3$ & 239.0 & $8^{n} \mathrm{Cs}$ & $02-21-85$ & \\
\hline RWM 9 & 104099.8 & 50400.0 & $\begin{array}{l}220.6-210.2 \\
200.0-189.6 \\
169.0-168.6 \\
143.0-192.6\end{array}$ & 977.6 & 380.6 & 248.0 & $8^{\circ} \mathrm{Cs}$ & $02.26-86$ & \\
\hline RWM 10 & 102000.9 & 48244.1 & $\begin{array}{l}216.5-205.1 \\
194.8-184.6 \\
158.6-148.2 \\
137.9-127.5\end{array}$ & 352.5 & 355.6 & 228.0 & $8^{n} \mathrm{Cs}$ & $02-21-85$ & \\
\hline RWM 11 & 104876.0 & 50400.2 & $\begin{array}{l}214.6-204.2 \\
198.9-188.6 \\
183.2-172.9 \\
152.2-141.9\end{array}$ & 380.3 & 383.3 & 241.4 & $8^{n} \mathrm{CS}$ & $02-26-85$ & \\
\hline RWM 12 & 106879.2 & 52500.1 & $\begin{array}{l}210.4-189.9 \\
179.7-169.2\end{array}$ & $\mathbf{3 5 9 . 0}$ & 359.4 & 200.2 & $6^{\circ} \mathrm{Cs} / \mathrm{ss}$ & $01-16-90$ & \\
\hline RWM 16 & 97647.2 & 48244.8 & $\begin{array}{l}188.4-168.0 \\
162.6-147.2\end{array}$ & 318.4 & 319.4 & & $6^{*} \mathrm{Cs} / \mathrm{BS}$ & $05-01.90$ & \\
\hline $\begin{array}{l}\text { RWM 16PA } \\
\text { RWM 16PB }\end{array}$ & $\begin{array}{l}97783.9 \\
97672.1\end{array}$ & $\begin{array}{l}48364.9 \\
48212.5\end{array}$ & $\begin{array}{l}188.9-148.8 \\
189.3-149.2\end{array}$ & $\begin{array}{l}327.9 \\
317.8\end{array}$ & $\begin{array}{l}328.7 \\
319.5\end{array}$ & $\begin{array}{l}179.9 \\
170.3\end{array}$ & $\begin{array}{l}2^{n} \text { PVC } \\
2^{n} \text { PVC }\end{array}$ & $\begin{array}{l}07-26-90 \\
07-26-90\end{array}$ & \\
\hline
\end{tabular}




\section{RWM WELL SERIES (cont.)}

Notes: The effective well depths for RWM 2 through 12 and RWM 16 are the distance from the top of casing to the bottom of the lowest screen zone.

RWM 1 has been referred to as MPT 1.

RWM 16PA and RWM 16PB are piezometers installed as observation wells for the pump test for RWM 16.

1 Plume definition well also required by the SRS Hazardous Waste Permit.

\section{S Well}

Description: Unofficial wells in S Area

Bldg. Number: Not applicable

Map: Not applicable

\begin{tabular}{|c|c|c|c|c|c|c|c|}
\hline & SRS Coordinates & Elevatione (ft MSL) & & Eff. Well & & & \\
\hline Well ID & North East & Screen Zone Grnd. & Casing & Depth (A) & Casing & Installed & Abandoned \\
\hline 8 & & & & & $2^{n}$ PVC & & $01-29-88$ \\
\hline
\end{tabular}

Notes: The identification of this well comes from the abandonment sheets. The 8 designation is unofficial. This unidentified well was drilled out (all casing and screen material removed), grouted, and abandoned due to S-Area construction.

\section{S WELL SERIES}

Description: Wells predating the construction of SRS

Bldg. Number: Not applicable

Map: Figure A-13

\begin{tabular}{|c|c|c|c|c|c|c|c|c|c|}
\hline \multirow[b]{2}{*}{ Well ID } & \multicolumn{2}{|c|}{ SRS Coordinates } & \multicolumn{3}{|c|}{ Elevations (ft MSL) } & \multirow{2}{*}{$\begin{array}{l}\text { Eff. Well } \\
\text { Depth (At) }\end{array}$} & \multirow[b]{2}{*}{ Casing } & \multirow[b]{2}{*}{ Installed } & \multirow[b]{2}{*}{ Abandoned } \\
\hline & North & East & Screen Zone & Grnd. & Casing & & & & \\
\hline 89 & 74919 & 53785 & & 257.5 & 260.0 & & & & \\
\hline 15 & 75381 & 63930 & & 264.6 & 267.0 & & & & \\
\hline 16 & 75223 & 63491 & & 259.3 & 261.8 & & & & \\
\hline
\end{tabular}

\section{SB WELL SERIES}

Description: Special Burial Wells in the Burial Grounds

Bldg. Number: 643-28E

Map: Not applicable

\begin{tabular}{|c|c|c|c|c|c|c|c|c|c|}
\hline \multirow[b]{2}{*}{ Well II } & \multicolumn{2}{|c|}{ SRS Coordinates } & \multicolumn{3}{|c|}{ Elevations (ft MSL) } & \multirow{2}{*}{$\begin{array}{l}\text { Eff. Well } \\
\text { Depth (ft) }\end{array}$} & \multirow[b]{2}{*}{ Casing } & \multirow[b]{2}{*}{ Installed } & \multirow[b]{2}{*}{ Abandoned } \\
\hline & North & East & Screen Zone & Grnd. & Casing & & & & \\
\hline BB 1 & & & & 295.8 & 298.8 & & 4" PVC & & $05-26-88$ \\
\hline BB 2 & & & & 295.4 & 298.4 & & 4" PVC & & $05-26-88$ \\
\hline SB & & & & 295.0 & 298.0 & & 4" PVC & & $05-26-88$ \\
\hline
\end{tabular}

(series continued) 
SB WELL SERIES (cont.)

\begin{tabular}{|c|c|c|c|c|c|c|c|c|c|}
\hline \multirow{2}{*}{\multicolumn{2}{|c|}{ Well ID }} & 8RS Coordinates & \multicolumn{3}{|c|}{ Elevations (At MSL) } & \multirow{2}{*}{$\begin{array}{l}\text { Eff. Well } \\
\text { Depth (t) }\end{array}$} & \multirow[b]{2}{*}{ Casing } & \multirow[b]{2}{*}{ Installed } & \multirow[b]{2}{*}{ Abandoned } \\
\hline & & North & Screen Zone & Grnd. & Casing & & & & \\
\hline 8B & 4 & & & 294.1 & 297.1 & & 4" PVC & & $05-26-88$ \\
\hline SB & 5 & & & 294.6 & 297.6 & & $4^{n}$ PVC & & $05-27-88$ \\
\hline SB & 6 & & & 294.7 & 297.7 & & $4^{n}$ PVC & & $05.27-88$ \\
\hline BB & 7 & & & 294.3 & 297.3 & & $4^{n}$ PVC & & $05-27.88$ \\
\hline SB & 8 & & & 293.5 & 296.6 & & $4^{n}$ PVC & & $05.25-88$ \\
\hline 8B & 9 & & & 293.4 & 296.4 & & $4^{n}$ PVC & & $0.5-25-88$ \\
\hline SB & 10 & & & 293.4 & 296.4 & & $4^{n}$ PVC & & $05-25-88$ \\
\hline 8B & 11 & & & 293.7 & 296.7 & & $4^{n}$ prC & & $05-25-88$ \\
\hline SB & 12 & & & 293.6 & 296.6 & & $4^{m} \mathrm{PVC}$ & & $05-25-88$ \\
\hline SB & 19 & & & 299.8 & 296.8 & & $4^{n}$ PVC & & 05.25 .88 \\
\hline 8B & 14 & & & 294.1 & 297.1 & & $4^{\prime \prime}$ PVC & & $05 \cdot 26-88$ \\
\hline $\mathbf{S B}$ & 15 & & & 292.5 & 295.5 & & $4^{n}$ Bteel & & $05-25-88$ \\
\hline SB & 16 & & & 291.6 & 294.6 & & $4^{n}$ Eteel & & $05.24-88$ \\
\hline SB & 17 & & & 291.2 & 294.2 & & $4^{n}$ Steel & & $05-24-88$ \\
\hline BB & 18 & & & 291.6 & 294.6 & & $4^{n}$ Bteel & & $05.29-88$ \\
\hline 8B & 19 & & & 290.2 & 293.2 & & $4^{n}$ Bteel & & $05.29-88$ \\
\hline SB & 20 & & & 290.1 & 298.1 & & $4^{n}$ Bteel & & $05.23-88$ \\
\hline
\end{tabular}

\section{SBA WELL SERIES}

The SBA Series was renamed the AMB Series in August 1984 according to Table A-1, "Prefix Letter and Project Series in AM Area," Marine and Bledsoe (1985). See the AMB Series.

\section{SBG WELL SERIES}

Description: S-Area Defense Waste Processing Facility (DWPF) Background Wells Bldg. Number: $\mathrm{N} \uparrow$ applicable

Map: Figures A-1 and A-16

\begin{tabular}{|c|c|c|c|c|c|c|c|c|c|}
\hline \multirow[b]{2}{*}{ Well ID } & \multicolumn{2}{|c|}{ BRs Coordinates } & \multicolumn{3}{|c|}{ Elevations (ft MSL) } & \multirow{2}{*}{$\begin{array}{l}\text { Dff. Well } \\
\text { Depth (A) }\end{array}$} & \multirow[b]{2}{*}{ Casing } & \multirow[b]{2}{*}{ Installed } & \multirow[b]{2}{*}{ Abandoned } \\
\hline & North & East & Screen Zone & Grnd. & Casing & & & & \\
\hline 8BG 1 & 74619.4 & 69749.1 & 220.7-190.7 & 260.7 & 262.4 & 71.7 & $4^{*}$ PVC & $09-19-86$ & \\
\hline SBG 2 & 74570.2 & 64939.6 & 235.9-205.9 & 287.9 & 290.0 & 84.1 & & $09-23-85$ & \\
\hline SBG 3 & 73699.9 & 65265.6 & $256.6-206.6$ & 284.6 & 286.6 & 80.0 & 4" PVC & $09-24-86$ & \\
\hline BBG 4 & 72999.8 & 66010.2 & $215.6-185.6$ & 271.6 & 273.1 & 87.5 & $4^{\prime \prime}$ & $09-25-85$ & \\
\hline SBG 6 & 72208.3 & 64499.0 & 219.4-199.4 & 282.4 & 284.6 & 86.1 & $4^{*}$ PVC & $01.16-87$ & \\
\hline 8BG 6 & 73599.3 & 63860.0 & 238.1.208.1 & 280.1 & 281.7 & 73.6 & 4" PVC & $09-20-85$ & \\
\hline
\end{tabular}




\section{SCA WELL SERIES}

Description: S-Area Vitrification Building

Bldg. Number: 221-S

Map: Figure A-16

\begin{tabular}{|c|c|c|c|c|c|c|c|c|c|}
\hline & gRs Cor & dinates & Elevations (f & MSL) & & Eff. Well & & & \\
\hline Well ID & North & Eagt & Screen Zone & Grnd. & Casing & Depth (ft) & Casing & Installed & Abandoned \\
\hline SCA 1 & & & & & & & $4^{n} 88$ & $05-02-86$ & \\
\hline $\begin{array}{l}\text { SCA } 1 A \\
\text { SCA } 2\end{array}$ & 73850.6 & 64697.1 & 245.9-216.9 & 286.9 & 288.9 & 73.0 & $\begin{array}{l}4^{n} 88 \\
4^{n} \mathrm{88}\end{array}$ & $\begin{array}{l}03-14-86 \\
01-04-86\end{array}$ & \\
\hline SCA $2 A$ & 73865.8 & 64701.0 & 271.2-266.2 & 286.9 & 288.9 & 22.7 & $4^{n} \mathrm{SS}$ & $01-02.86$ & \\
\hline SCA 3 & 73959.3 & 64571.2 & $240.3-220.3$ & 285.3 & 287.3 & 67.0 & $4^{n}$ PVC & $12-22-89$ & \\
\hline SCA $\mathbf{3 A}$ & 73965.0 & 64571.2 & 277.1-267.1 & 286.3 & 287.3 & 20.2 & 4" PVC & $12-15-89$ & \\
\hline SCA 4 & 73856.5 & 64563.5 & $240.4-220.4$ & 283.9 & 286.2 & 65.8 & $4^{n}$ PVC & $12-21.89$ & \\
\hline SCA $4 A$ & 73855.2 & 64567.2 & 275.9-265.3 & 283.9 & 286.1 & 20.8 & 4" PVC & $12-18-89$ & \\
\hline SCA 6 & 74092.9 & 64630.8 & 243.7-223.7 & 286.1 & 288.1 & 64.4 & $4^{n}$ PVC & 01.03 .90 & \\
\hline 8CA 6 & 73706.2 & 64687.6 & 241.1-221.3 & 283.8 & 285.8 & 64.6 & 4" PVC & $01-10.90$ & \\
\hline
\end{tabular}

\section{SDS WELL SERIES}

Description: Z-Area Saltstone Disposal Site Facility Bldg. Number: 704-Z

Map: Figure A-16

\begin{tabular}{|c|c|c|c|c|c|c|c|c|c|}
\hline \multirow[b]{2}{*}{ Well ID } & \multicolumn{2}{|c|}{ SRS Coordinater } & \multicolumn{3}{|c|}{ Plevations ( $\mathrm{A}$ MSL) } & \multirow{2}{*}{$\begin{array}{l}\text { Eff. Woll } \\
\text { Depth (ft) }\end{array}$} & \multirow[b]{2}{*}{ Casing } & \multirow[b]{2}{*}{ Installed } & \multirow[b]{2}{*}{ Abandoned } \\
\hline & North & East & Screen Zone & Grnd. & Casing & & & & \\
\hline 8DS 1 & 75900 & 66400.0 & & & & & 4" PVC & $11-15.79$ & $04-27-87$ \\
\hline SDB 2 & 77286.4 & 64923.2 & 237.6-217.6 & 287.6 & 289.6 & 72.0 & $4^{n}$ PVC & $04-03-81$ & $05.28-87$ \\
\hline SDS BA & 76643.4 & 64711.4 & $230.6-210.6$ & 290.5 & 292.5 & 82.0 & $4^{n}$ PVC & $04-02-81$ & $06-05-87$ \\
\hline SDS 4 & 75816.6 & 63722.5 & $206.4-186.4$ & 256.4 & 257.4 & 72.0 & 4" PVC & $08-25-81$ & $08-04-87$ \\
\hline SDS 4A & 76803.9 & 63726.0 & 233.4-228.4 & 253.4 & 267.6 & 29.2 & $4^{n}$ PrC & $05-06-81$ & $08-04-87$ \\
\hline SDS 5 & 75935.3 & 66712.5 & 238.2-218.2 & 288.2 & 289.8 & 71.6 & 4" PVC & $04-24-81$ & 09-10-87 \\
\hline SDB 5A & & & & & & & $4^{n}$ Stwel & & $03-08-87$ \\
\hline SDS 6 & 76862.7 & 66991.8 & 236.9-216.9 & 286.9 & 290.0 & 73.1 & 4" PVC & $04-11-81$ & $04-20-87$ \\
\hline SDS 7A & 76515.7 & 67681.0 & $80.0-75.0$ & 274.0 & 276.6 & 201.6 & 4" Stoel & $04-23-81$ & $01-27-88$ \\
\hline 8DS $7 B$ & 76518.2 & 67686.2 & $140.3-135.3$ & 275.3 & 278.0 & 142.7 & 4" Bteel & $04-13-81$ & $01-21-88$ \\
\hline SDS 7C & 76552.7 & 67694.0 & $189.2-184.2$ & 274.2 & 277.2 & 93.0 & $4^{n}$ Steel & $04-11.81$ & $01-16-88$ \\
\hline SDS TD & 76527.4 & 67701.7 & 225.6-205.6 & 275.6 & 277.2 & 71.6 & $4^{n}$ Steel & $04-11.81$ & $01.13-88$ \\
\hline SDS 8 & 75399.8 & 67530.0 & 221.9-201.9 & 288.9 & 272.1 & 70.2 & 4" PVC & $04-10-81$ & $04-22-87$ \\
\hline SDS 9 & 74819.4 & 66178.5 & 243.1-223.1 & 293.1 & 295.7 & 72.6 & $4^{n}$ PVC & $04-09-81$ & $09-25-87$ \\
\hline SDB 10 & 76572.0 & 65770.1 & 246.7-225.7 & 291.7 & 295.2 & 69.6 & $4^{n}$ PVC & $04-10.81$ & $09-29-87$ \\
\hline SDS 11 & 76410.9 & 65563.8 & 239.2-219.3 & 289.3 & 291.9 & 72.6 & 4" PVC & $04-09-81$ & $04-29-87$ \\
\hline SDS 12A & 77741.6 & 66610.6 & $141.4-136.4$ & 276.4 & 278.7 & 142.3 & $4^{n}$ Bteel & $03-27.81$ & $01.11-88$ \\
\hline SDS 12B & 77749.9 & 66610.7 & 191.7-186.7 & 276.7 & 278.7 & 92.0 & $4^{n}$ Steel & $03-31-81$ & $12-23-87$ \\
\hline SDS 12C & 77759.1 & 66610.4 & 226.1-206.1 & 276.1 & 279.1 & 73.0 & $4^{n}$ Steel & $04-01.81$ & $12-17-87$ \\
\hline SDB 13A & 78900.0 & 66642.9 & $224.4-204.4$ & 274.4 & 277.4 & 73.0 & $4^{n}$ PVC & $04-03-81$ & $04-30-87$ \\
\hline SDS 14 & 77872.6 & 67572.9 & $230.4-210.4$ & 270.4 & 272.4 & 62.0 & $4^{n}$ PVC & $04-06-81$ & $05-21-87$ \\
\hline SDS 14A & 77872.4 & 67577.1 & $257.8-252.8$ & 267.8 & 271.0 & 18.2 & $4^{n}$ PVC & $05-05-81$ & $05.20-87$ \\
\hline SDS 16 & 77276.2 & 63979.7 & $232.0-212.0$ & 282.0 & 284.9 & 72.9 & $4^{n}$ PVC & $03-24-81$ & $01-27-88$ \\
\hline SDS 16A & 76420.9 & 68789.6 & 206.1-186.1 & 231.1 & 294.3 & 48.2 & $4^{n}$ PVC & $04-07-81$ & \\
\hline SDS 16B & 76510 & 68787 & & & & & $4^{\prime \prime}$ PVC & & $05-14-87$ \\
\hline SDS 16C & 76512 & 68781 & & & & & 4" PVC & & $05-12-87$ \\
\hline SDS 17 & 74420.6 & 67353.7 & 216.6-196.6 & 267.6 & 271.3 & 74.7 & $4^{n}$ PVC & $04-08-81$ & $05-05-87$ \\
\hline
\end{tabular}


SDS WELL SLRIES (cont.)

\begin{tabular}{|c|c|c|c|c|c|c|c|c|c|}
\hline \multirow[b]{2}{*}{ Well ID } & \multicolumn{2}{|c|}{ BRS Coordinates } & \multicolumn{3}{|c|}{ Elevations (ft MSL) } & Eff. Well & \multirow[b]{2}{*}{ Casing } & \multirow[b]{2}{*}{ Installed } & \multirow[b]{2}{*}{ Abandone } \\
\hline & North & East & Screen Zone & Grnd. & Casing & Depth (t) & & & \\
\hline SD8 18 & 77564.2 & 68278.7 & 235.3-215.3 & 265.3 & 267.6 & 62.3 & $4^{\prime \prime}$ PVC & $04-08-81$ & $05-22-87$ \\
\hline SDS 18A & 77579 & 68280 & & & & & $4^{\prime \prime}$ PVC & & $05-26-87$ \\
\hline SDS 19 & 74937.5 & 68173.6 & 212.8-192.8 & 293.8 & 236.1 & 43.3 & 4" PVC & $04-07-81$ & $07-10-86$ \\
\hline $\begin{array}{l}\text { SDS 20A } \\
\text { SDS 20B }\end{array}$ & $\begin{array}{l}77928 \\
77925\end{array}$ & $\begin{array}{l}67309 \\
67302\end{array}$ & & & & & $\begin{array}{l}4^{n} \text { PVC } \\
4^{n} \text { PVC }\end{array}$ & & $\begin{array}{l}05-11-87 \\
05-03-87\end{array}$ \\
\hline SDS 20C & 77927 & 67295 & & & & & $4^{n}$ PVC & & $05-06-87$ \\
\hline $\operatorname{SDS} 21 \mathrm{~A}$ & 78951 & 67087 & & & & & 4" PVC & & $05-15-87$ \\
\hline SDS 21B & 78949 & 67080 & & & & & $4^{n}$ PVC & & $05-07-87$ \\
\hline $\operatorname{sDg} 21 \mathrm{C}$ & 78946 & 67074 & & & & & $4^{n}$ PVC & & $05-13-87$ \\
\hline SDS 22A & 76887 & 66304 & & & & & $4^{\prime \prime}$ PVC & & $04.09-87$ \\
\hline SDS 22B & 76895 & 66302 & & & & & $4^{n}$ PVC & & $04-13-87$ \\
\hline SDS $22 \mathrm{C}$ & 76902 & 66300 & & & & & 4" PVC & & $04-14-87$ \\
\hline
\end{tabular}

Notes: D'Appolonia Consulting Engineers took boring samples from most of the well holes drilled in the 8DS Series. Boringe 8Ds 13 and 16 were abandoned, and wells were inetalled nearby. These wells were designated 8DS 13A and 8DS 16A in D'Appolonia (n.d.). There are conflicting coordinates and casing length reports for SDS 161. 8 DS 3 is designated gDs $\mathrm{s}$.

\section{SDS 124 Well}

Description: Unofficial well in Z Area

Bldg. Number: Not applicable

Map: Figure A-16

\begin{tabular}{|c|c|c|c|c|c|c|c|c|}
\hline & SRS & dinates & Hevations (ft MSL) & & Eff. Well & & & \\
\hline Well ID & North & East & Screen Zone Grnd. & Casing & Depth (ft & Casing & Installed & Abandoned \\
\hline DS12 & 78920 & 68666 & & & & 4" PVC & & $05-19-87$ \\
\hline
\end{tabular}

Notes: The identification of this well comes from the absndonment sheets. The $8 D 8124$ designation is unofficial. OBs 2 and BDB 124 have been thought to be the same well in the past. However, the Daily Activity Reports (DAR) accompanying the abandonment report for 8DS 124 say the welle are not the same. One note states that, OBS 2 was not found. A cecond note says that drillers found 8DS 124 and that "OBS 2 is merely o stake in the ground." In addition, the DAR Bay that the 8188 wells only run through number 22 and that the coordinates for BDS 124 do not align with other 8DS wells. A final note on the DAR for $8 D S$ 124 (5-19-87) says that "8DS 124 was written on side of PVC casing but covered with paper label marked as OBS 2."

\section{SLP WELL SERIES}

Description: S-Area. Low Point Pump Pit

Bldg. Number: 511-S

Map: Figure A-16

\begin{tabular}{|c|c|c|c|c|c|c|c|c|c|}
\hline \multirow[b]{2}{*}{ Well ID } & \multicolumn{2}{|c|}{ BRS Coordinates } & \multicolumn{3}{|c|}{ Elevations (ft MSL) } & \multirow{2}{*}{$\begin{array}{l}\text { Eff. Well } \\
\text { Depth (tt) }\end{array}$} & \multirow[b]{2}{*}{ Casing } & \multirow[b]{2}{*}{ Installed } & \multirow[b]{2}{*}{ Abandoned } \\
\hline & North & Fast & Screen Zone & Grnd. & Casing & & & & \\
\hline SLP 1 & 72958.4 & 64449.1 & 248.0-228.0 & 283.0 & 284.8 & 56.8 & 4" PVC & $11-09-87$ & \\
\hline SLP 2 & 72863.4 & 64529.7 & 237.7-217.7 & 281.8 & 283.8 & 66.1 & $4^{\prime \prime}$ PVC & $11-06-87$ & \\
\hline
\end{tabular}




\section{SLW WELL SERIES}

Description: New Sanitary Landfill Piezometer Wells

Bldg. Number: Not applicable

Map: Figure A-24

\begin{tabular}{|c|c|c|c|c|c|c|c|c|c|}
\hline \multirow[b]{2}{*}{ Well ID } & \multicolumn{2}{|c|}{ BRS Coordinates } & \multicolumn{3}{|c|}{ Elevations (ft MSL) } & \multirow{2}{*}{$\begin{array}{l}\text { Eff. Well } \\
\text { Depth (t) }\end{array}$} & \multirow[b]{2}{*}{ Casing } & \multirow[b]{2}{*}{ Installed } & \multirow[b]{2}{*}{ Abandoned } \\
\hline & North & East & Screen Zone & Grnd. & Casing & & & & \\
\hline SLW 1 & 87603.6 & 40299.8 & $180.0-160.0$ & 302.0 & 304.1 & 144.1 & $2^{n}$ PVC & $04-17-88$ & \\
\hline SLW 2 & 88690.5 & 40105.0 & $197.0-171.9$ & 302.3 & 304.6 & 132.7 & 2" PVC & $05-05.88$ & \\
\hline SLW 3 & 88225.9 & 99205.5 & 188.7-168.7 & 276.7 & 278.7 & 110.0 & $2^{n}$ PVC & $05-13-88$ & \\
\hline SLW 4 & 86999.9 & 39199.9 & 189.2-169.2 & 299.2 & 300.7 & 131.6 & $2^{n}$ PVC & $05-02-88$ & \\
\hline SLW 5 & 87602.3 & 38105.7 & $194.6-174.6$ & 299.6 & 242.0 & 67.4 & 2" PVC & $04-27-88$ & \\
\hline SLW 6 & 86797.0 & 38199.4 & 199.1-179.1 & 249.1 & 251.5 & 72.4 & 2" PVC & $04-27.88$ & \\
\hline SLW 7 & 88303.9 & 38100.8 & 174.1-164.1 & 229.1 & 231.1 & 77.0 & $2^{n}$ PVC & $04-21.88$ & \\
\hline SLW 8 & 89267.2 & 38738.6 & $195.3-165.3$ & 255.3 & 257.5 & 92.2 & 2" PVC & $04-25-88$ & \\
\hline
\end{tabular}

\section{SR WELL SERIES}

The SR Series was renamed the SRW Series in September 1984 according to Table A-1, "Prefix Letter and Project Series in A/M Area," Marine and Bledsoe (1985).

\section{SRW WELL SERIES}

Description: Silverton Road Waste Site

Bldg. Number: 731-3A

Map: Figure A-17

\begin{tabular}{|c|c|c|c|c|c|c|c|c|c|}
\hline \multirow[b]{2}{*}{ Well D } & \multicolumn{2}{|c|}{ BRS Coordinates } & \multicolumn{3}{|c|}{ Elevations (ft MSL) } & \multirow{2}{*}{$\begin{array}{l}\text { Fff. Well } \\
\text { Depth (At) }\end{array}$} & \multirow[b]{2}{*}{ Casing } & \multirow[b]{2}{*}{ Intalled } & \multirow[b]{2}{*}{ Abandoned } \\
\hline & North & East & Screen Zone & Grod. & Casing & & & & \\
\hline SRW 1 & 103776.7 & 41407.0 & $230.2-200.2$ & 313.2 & 315.? & 115.0 & 4" PVC & $10-21-81$ & \\
\hline SRW 2 & 103721.8 & 41627.2 & 228.6-198.6 & 318.6 & 920.6 & 122.0 & 4" PVC & $10-22-81$ & \\
\hline SRW 2A & 103720.8 & 41694.6 & 98.488 .6 & 319.1 & 320.6 & 292.0 & 4" PVC & $08-08-83$ & \\
\hline SRW 2B & 103729.7 & 41631.7 & $162.6-162.8$ & 319.2 & 320.6 & 167.8 & 4" PVC & $12-20-83$ & \\
\hline SRW 3 & 103530.5 & 41843.4 & 298.4-208.4 & 329.4 & 331.4 & 123.0 & 4" PVC & $10-27-81$ & $08-20-84$ \\
\hline SRW 3A & 103516.4 & 41851.2 & 193.1-163.1 & 330.1 & 332.1 & 169.0 & $4^{n}$ PVC & $08-20-84$ & \\
\hline SRW 4 & 103959.9 & 41612.4 & 230.1-200.1 & 318.1 & 320.1 & 120.0 & $4^{n}$ PVC & $09-28-81$ & \\
\hline SRW 5 & 103418.2 & 41240.0 & 224.6-194.6 & 307.6 & 309.4 & 114.8 & $4^{n}$ PVC & $10.29-81$ & \\
\hline SRW 6 & 103602.7 & 41243.9 & 222.6-192.6 & 305.6 & 307.7 & 115.1 & 4" PVC & $11-09-81$ & \\
\hline SRW 7 & 103541.5 & 40926.2 & 217.5-197.5 & 296.7 & 299.1 & 101.6 & $4^{n}$ PVC & $05-03-82$ & \\
\hline SRW 8 & 109470.0 & 40465.9 & 216.7-195.7 & 286.7 & 288.1 & 92.4 & 4" PVC & $05-10-82$ & \\
\hline SRW 9 & 103259.8 & 39688.4 & $196.3-166.3$ & 251.3 & 253.4 & 87.1 & $4^{\prime \prime}$ PVC & $07-20-82$ & \\
\hline SRW 9A & 103251.1 & 39692.9 & $124.3-114.4$ & 251.4 & 253.3 & 138.9 & $4^{\prime \prime}$ PVC & $08-21.83$ & \\
\hline SRW 9B & 103241.6 & 39697.6 & 162.4-152.6 & 251.7 & 253.4 & 100.8 & $4^{\prime \prime}$ PVC & $12.19-83$ & \\
\hline SRW 10 & 103387.9 & 40944.3 & 223.0-193.0 & 301.0 & 303.4 & 110.4 & 4" PVC & $09-16-82$ & \\
\hline SRW 11 & 103693.2 & 40874.2 & $220.6-190.6$ & 293.6 & 295.8 & 105.2 & 4" PVC & $09-20-82$ & \\
\hline SRW 12A & 103710.3 & 39013.3 & $113.7-103.9$ & 234.1 & 286.3 & 132.4 & $4^{\prime \prime}$ PVC & $09-20-83$ & \\
\hline SRW 12B & 103702.7 & 39020.3 & 156.1-146.3 & 234.1 & 236.3 & 90.0 & $4^{n}$ PVC & $12-17-83$ & \\
\hline SRW 12C & 103712.5 & 39023.1 & 198.9-179.1 & 294.3 & 236.3 & 67.2 & 4" PVC & $09-30-83$ & \\
\hline SRW 13A & 108001.0 & 40668.2 & 103.6- 93.8 & 295.7 & 297.7 & 203.9 & $4^{\prime \prime}$ PVC & $12-05-83$ & \\
\hline SRW 13B & 102993.6 & 40675.8 & $163.2-163.3$ & 295.7 & 297.7 & 144.4 & $4^{n}$ PVC & $12-16-83$ & \\
\hline
\end{tabular}

(series continued) 
SRW WELL SERIES (cont.)

\begin{tabular}{|c|c|c|c|c|c|c|c|c|c|}
\hline \multirow[b]{2}{*}{ Well ID } & \multicolumn{2}{|c|}{ 8R8 Coordinates } & \multicolumn{3}{|c|}{ Elevations (ft MSL) } & \multirow{2}{*}{$\begin{array}{l}\text { Eff. Well } \\
\text { Depth (ft) }\end{array}$} & \multirow[b]{2}{*}{ Casing } & \multirow[b]{2}{*}{ Inotalled } & \multirow[b]{2}{*}{ Abandoned } \\
\hline & North & East & Screen Zone & Grnd. & Casing & & & & \\
\hline BRW 13C & 102988.5 & 40682.7 & $225.4-195.8$ & 295.9 & 297.7 & 101.9 & $4^{n}$ PVC & $12-19-83$ & \\
\hline SRW 14A & 102831.3 & 41538.6 & $\begin{array}{l}123.7-113.9 \\
110.9-107.9\end{array}$ & 324.9 & 327.0 & 213.1 & 4" PVC & $09.03-83$ & \\
\hline SRW 14B & 102836.1 & 41548.1 & $162.9-153.1$ & 324.9 & 326.9 & 173.8 & $4^{n}$ PVC & $12-16.83$ & \\
\hline SRW 14C & 102824.2 & 41546.4 & 228.3-198.6 & 325.3 & 326.9 & 128.3 & 4" PVC & $11-15-83$ & \\
\hline SRW 15A & 104778.0 & 41234.7 & 107.6- 97.8 & 317.4 & 319.1 & 221.3 & $4^{n}$ PVC & 10.02 .83 & \\
\hline SRW 16B & 104772.9 & 41252.5 & $161.6-151.8$ & 317.1 & 319.1 & 167.3 & $4^{n}$ PVC & 11.12 .83 & \\
\hline SRW 15C & 104774.9 & 41245.1 & $217.9-187.7$ & 317.3 & 319.1 & 131.4 & $4^{n}$ PVC & $10.18-83$ & \\
\hline SRW 16A & 103769.4 & 42830.9 & $144.1-119.4$ & 344.5 & 346.8 & 227.4 & $4^{\prime \prime}$ PVC & $10-28-83$ & \\
\hline SRW 16B & 103772.0 & 42826.8 & $169.9-160.1$ & 344.4 & 346.8 & 186.7 & 4" PVC & $11-13-83$ & \\
\hline SRW 16C & 103772.4 & 42841.8 & 235.7-205.7 & 346.3 & 346.6 & 140.9 & 4" PVC & $07-04-84$ & \\
\hline
\end{tabular}

Notes: The 82 Series was renamed the $8 R$ W Series in September 1984 according to Table A-1, "Prefix Lotter and Project Berie日 in AM Area, " Marine and Bledsoe (1986).

According to the Drilling Request, the firet ERW 10 well was "backfilled with cement because of incorrect inetallation," and the second $8 \mathrm{RW} 10$ well was drilled $10 \mathrm{ft}$ from the first well.

8BW $14 A$ has two screons.

\section{SSS WELL SERIES}

Description: Sewage Sludge Application Sites-see below for specific locations

Bldg. Number: $761-G,-1 G,-2 G,-3 G,-4 G,-5 G,-6 G,-7 G,-8 G$

Map: Figures A-4, A-13, A-24, A-25, A-26, A-27, and A-28

\begin{tabular}{|c|c|c|c|c|c|c|c|c|c|}
\hline & SRS Coc & linates & Elevations (f & MSL) & & Eff. Well & & & \\
\hline Well ID & North & Eagt & Screen Zone & Grnd. & Casing & Depth (ft) & Caging & Ingtalled & Abandoned \\
\hline 8881 & 54219.6 & 28968.8 & & 192.1 & 194.9 & & & $11-04-80$ & \\
\hline 8882 & 53846.7 & 27935.0 & & 162.1 & 165.1 & & & 11.0480 & \\
\hline 8853 & 53128.9 & 28216.6 & & 159.8 & 163.6 & & & $11-04-80$ & \\
\hline 8984 & 84842.7 & 39298.6 & & 265.6 & 266.8 & & & & \\
\hline 8885 & 82790.7 & 38698.7 & & 239.3 & 240.0 & & & $11-04-80$ & \\
\hline 8886 & 82482.2 & 39743.2 & & 212.4 & 213.4 & & & $11-04-80$ & \\
\hline 8887 & 84512.8 & 43286.0 & & 226.3 & 226.6 & & & $11-04-80$ & \\
\hline 8888 & 84276.7 & 43897.7 & & 200.1 & 201.2 & & & $11-0480$ & \\
\hline $88^{\prime} \cdot 9$ & 84636.4 & 43949.1 & & 201.9 & 203.3 & & & $11-04-80$ & \\
\hline 89810 & 94196.0 & 58105.6 & & 309.7 & 311.6 & & & $11-04-80$ & \\
\hline 88811 & 93813.0 & 59015.8 & & 302.4 & 304.1 & & & $11-04-80$ & \\
\hline 88812 & 94044.1 & 59246.4 & & 299.9 & 302.3 & & & $11-04-80$ & \\
\hline 88813 & 47758.8 & 40220.3 & & 227.5 & 228.6 & & 2" PVC & $11.04-80$ & $11-03-88$ \\
\hline 88814 & 46803.8 & 40438.4 & & 189.8 & 191.1 & & $2^{n}$ PVC & $11-04-80$ & $11-03-88$ \\
\hline $\operatorname{SsS} 15$ & 46644.4 & 40749.2 & & 182.5 & 184.3 & & 2" PVC & $11.04-80$ & $11-08-88$ \\
\hline 88816 & 36038.0 & 75910.3 & & 226.4 & 228.0 & & $2^{n}$ PVC & $11-04-80$ & $11-10-88$ \\
\hline S8S 17 & 36539.5 & 76518.2 & & 221.0 & 222.7 & & & $11-04-80$ & \\
\hline 88818 & 37298.2 & 75773.2 & & 217.3 & 218.6 & & $2^{n}$ PVC & 11.04 .80 & 11.09 .88 \\
\hline 88819 & 83978.9 & 41113.2 & & 227.5 & 230.2 & & & & \\
\hline 88820 & 84490.9 & 41469.7 & & 250.1 & 253.0 & & & & \\
\hline 88821 & 86053.0 & 40518.4 & & 283.0 & 286.5 & & & & \\
\hline 88822 & 91202.7 & 66003.8 & & 290.0 & 290.9 & & & & \\
\hline
\end{tabular}

(series continued) 
SSS WELL SERIES (cont.)

\begin{tabular}{|c|c|c|c|c|c|}
\hline \multirow[b]{2}{*}{ Well ID } & \multicolumn{2}{|c|}{ SRS Coordinates } & \multicolumn{2}{|c|}{ Elevations (ft MSL) } & \multirow[b]{2}{*}{ Casing } \\
\hline & North & East & Screen Zone & Grnd. & \\
\hline S8s 23 & 93429.C & 65994.1 & & 299.4 & 301.0 \\
\hline 88824 & 94097.7 & 65785.0 & & 313.3 & 316.2 \\
\hline S88 26 & 34385.6 & 83709.7 & & 202.1 & 203.2 \\
\hline S8S 26 & 34059.2 & 83755.1 & & 214.3 & 214.6 \\
\hline S8s 27 & 33495.8 & 84747.7 & & 231.5 & 213.8 \\
\hline SSS 29 & 74960.2 & 53548.1 & & & \\
\hline S8S 30 & 75537.8 & 52876.1 & & & \\
\hline \multicolumn{6}{|l|}{ S8S 31} \\
\hline \multicolumn{6}{|l|}{ SS8 32} \\
\hline \multicolumn{6}{|l|}{ SSS 33} \\
\hline \multicolumn{3}{|l|}{ Location } & \multicolumn{2}{|c|}{ Building No. } & Well No. \\
\hline \multirow{4}{*}{\multicolumn{3}{|c|}{$\begin{array}{l}\text { F-Area Sludge Land Application Site } \\
\text { K-Area Borrow Pit } \\
\text { Kato Road Site } \\
\text { Lower Kato Road Site }\end{array}$}} & \multicolumn{2}{|l|}{-} & 29.30 \\
\hline & & & \multicolumn{2}{|c|}{$761-4 G$} & $13-15$ \\
\hline & & & \multicolumn{2}{|c|}{$761.6 \mathrm{G}$} & $19-21$ \\
\hline & & & \multicolumn{2}{|c|}{$761-1 G$} & 46 \\
\hline \multicolumn{3}{|c|}{ Orangeburg Site } & \multicolumn{2}{|c|}{$761-2 G$} & 7.9 \\
\hline \multicolumn{3}{|c|}{ Par Pond Borrow Pit } & \multicolumn{2}{|c|}{$761-5 G$} & $16-18$ \\
\hline \multicolumn{3}{|c|}{ Road F Site } & \multicolumn{2}{|c|}{$761.7 G$} & 22.24 \\
\hline \multicolumn{3}{|c|}{ Sandy (Lucy) Site } & & 10.12 \\
\hline \multirow{2}{*}{\multicolumn{3}{|c|}{$\begin{array}{l}\text { Second Par Pond Borrow Pit } \\
\text { 40.Acre Hardwood Site }\end{array}$}} & \multirow{2}{*}{\multicolumn{2}{|c|}{$\begin{array}{l}761-8 G \\
761-G\end{array}$}} & 25.27 \\
\hline & & & & & 1.3 \\
\hline
\end{tabular}

T 18 N WELL SERIES (see NTN WELL SERIES)

T 18 S WELL SERIES (see NTS WELL SERIES)

T 18 W WELL SERIES (see NTW WELL SERIES)

\section{TBG WELL SERIES}

Description: TNX Burying Ground

Bldg. Number: $643-5 T$

Map: Figure A-29

\begin{tabular}{|c|c|c|c|c|c|c|c|c|}
\hline \multirow[b]{2}{*}{ Well ID } & \multicolumn{2}{|c|}{ SR8 Coordinates } & \multicolumn{3}{|c|}{ Elevations (ft MSL) } & \multirow{2}{*}{$\begin{array}{l}\text { Eff. Well } \\
\text { Depth (A) }\end{array}$} & \multirow[b]{2}{*}{ Casing } & \multirow[b]{2}{*}{ Installed } \\
\hline & North & Eagt & Screen Zone & Grnd. & Casing & & & \\
\hline TBG 1 & 71429.5 & 17134.7 & 109.1-89.1 & 149.1 & 151.2 & 62.1 & 4" PVC & $09-21.88$ \\
\hline TBG 3 & 71324.1 & 17177.7 & 108.9.88.9 & 148.9 & 151.2 & 62.3 & $4^{n} \mathrm{PVC}$ & $09-14-88$ \\
\hline TBG 4 & 71267.1 & $\mathbf{1 7 1 7 7 . 7}$ & 109.3-89.3 & 149.3 & 151.3 & 62.0 & 4" PVC & $09-19-88$ \\
\hline TBG 5 & 71226.5 & 17354.5 & $112.4-92.4$ & 147.4 & 149.4 & 57.0 & $4^{n}$ PVC & $09-01-88$ \\
\hline TBG $5 A$ & 71216.8 & 17354.8 & $56.2-46.2$ & 147.2 & 149.4 & 103.2 & $* *$ & $11-09-89$ \\
\hline TBG $6 B$ & 71206.8 & 17348.8 & $80.0-70.0$ & 147.8 & 150.0 & 80.0 & 4" PVC & 11.09 .89 \\
\hline
\end{tabular}

Eff. Well

Depth (ft) Casing Installed Abandoned
10-10-88

10-11.88

10-27.88

10-14-88

$10-20-88$ 
TBG WELL SERIES (cont.)

\begin{tabular}{|c|c|c|c|c|c|c|c|c|c|}
\hline \multirow[b]{2}{*}{ Well ID } & \multicolumn{2}{|c|}{ BRs Coordinates } & \multicolumn{3}{|c|}{ Elevations (ft MSL) } & \multirow{2}{*}{$\begin{array}{l}\text { Eff. Well } \\
\text { Depth (ft) }\end{array}$} & \multirow[b]{2}{*}{ Caring } & \multirow[b]{2}{*}{ Installed } & \multirow[b]{2}{*}{ Abandoned } \\
\hline & North & Fast & Screen Zone & Grnd. & Casing & & & & \\
\hline $\begin{array}{ll}\text { IBBG } 6 \\
\text { IBBG } 7\end{array}$ & 71482.3 & 17290.6 & 109.1-89.1 & 145.9 & 148.1 & 59.0 & $4^{n}$ PVC & $09-12-88$ & \\
\hline
\end{tabular}

Notes: * TBG $6 A$ has 4-in. PVC casing inside 12-in. PVC casing. The screen is also PVC.

\section{TNX WELL SERIES}

Description: TNX-Area Assessment Wells

Bldg. Number: Not applicable

Map: Figure A-29

\begin{tabular}{|c|c|c|}
\hline \multirow[b]{2}{*}{ WellID } & \multicolumn{2}{|c|}{ SRS Coordinates } \\
\hline & North & East \\
\hline NX 1D & 71613.6 & 16699.6 \\
\hline $2 D$ & 71462.0 & 16788.2 \\
\hline NX 3D & 71236.7 & 17043.1 \\
\hline$N X$ \&D & 71002.7 & 17229.0 \\
\hline$N X$ 6D & 70995.3 & 17363.7 \\
\hline NX 6D & 70717.6 & 17428.7 \\
\hline NX TD & 71738.1 & 17080.6 \\
\hline NX 8D & 70691.9 & 16168.3 \\
\hline INX 9D & 70791.4 & 16145.8 \\
\hline TNX 10D & 70999.3 & 16168.7 \\
\hline TNX 111 & 71199.3 & 16166.6 \\
\hline NX 12I & 71698.3 & 16176.3 \\
\hline
\end{tabular}

\begin{tabular}{|c|c|c|}
\hline \multicolumn{2}{|c|}{ Plevatione $(t \mathrm{MBI})$} & \\
\hline & Grnd. & Canin \\
\hline $99.6-79.6$ & 164.1 & 166.5 \\
\hline $102.8-82.8$ & 152.8 & 155.1 \\
\hline $104.9-84.9$ & 151.9 & 164.3 \\
\hline $106.6-86.5$ & 147.6 & 149.8 \\
\hline 108.5.88.5 & 147.0 & 149.3 \\
\hline $109.8-89.8$ & 148.2 & 150.5 \\
\hline $109.6-89.6$ & 148.6 & 160.9 \\
\hline $94.0-74.0$ & 98.0 & 100.3 \\
\hline $95.4-76.4$ & 99.4 & 101.7 \\
\hline $97.0-77.0$ & 100.0 & 102.3 \\
\hline $93.2-79.2$ & 97.7 & 99.8 \\
\hline & & \\
\hline
\end{tabular}

\section{TW WELL SERIES}

Description: Upper Tank Farm

Bidg. Number: 241-H

Map: Figures A-8, A-9, and A-11

\begin{tabular}{|c|c|c|}
\hline \multirow[b]{2}{*}{ Well ID } & \multicolumn{2}{|c|}{ SR8 Coordinates } \\
\hline & North & East \\
\hline W 1 & 75363.4 & 54 \\
\hline s & 74958.2 & \\
\hline $\mathbf{w}$ & 74786.3 & 56616. \\
\hline 5 & 74608.3 & 56906.9 \\
\hline $\mathbf{w}$ & 74413.5 & 66227 \\
\hline
\end{tabular}

Frf. Well

Depth (t) Casing Inotalled Abandoned

\begin{tabular}{|c|c|c|}
\hline 76.9 & $4^{*}$ PVC & $10.06-89$ \\
\hline 72.3 & 4" PVC & $10-31.89$ \\
\hline 69.4 & 4" PVC & $10-16-89$ \\
\hline 64.3 & $4^{*}$ PVC & $10-13-89$ \\
\hline 60.8 & $4^{*}$ PVC & 10.11 .89 \\
\hline 60.7 & $4^{*}$ PVC & $10-11.89$ \\
\hline 67.3 & $4^{*}$ PVC & $10-09-89$ \\
\hline 28.3 & 4" PVC & $10.17-89$ \\
\hline 26.3 & $4^{n}$ PVC & $10-19-89$ \\
\hline 25.3 & 4" PVC & $10-19-89$ \\
\hline 26.6 & & $10-20-89$ \\
\hline 26.1 & $4^{*}$ PVC & $10-20-89$ \\
\hline
\end{tabular}

RRf. Well

Depth (t) Casing Installed Abandoned

$\begin{array}{lll}\text { Stoel } & 07.01-60 \sim 01-01-81 * \\ \text { Stoel } & 07.01 .60 \sim & \\ \text { Stoel } & 07.01 .60 \sim 01.01 .81 * \\ \text { Stoel } & 07.01 .60 \sim & \\ \text { Steel } & 03.01 .61 \sim\end{array}$




\section{Unnamed Well}

Description: Unuamed well inside BG wells at the MWMF

Bldg. Number: Not applicable

Map: Not applicable

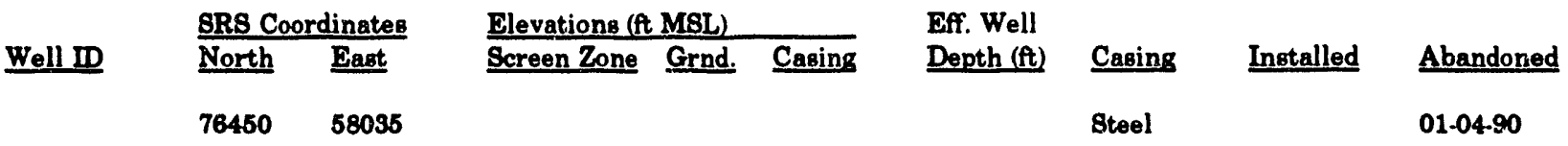

Notes: The description of this well comes from the abandoninent sheets. The Unnamed Woll designation is unofficial. The Daily Activity Report for this well locates it "inside BG Wells."

\section{WW WELL SERIES}

Description: Burial Grounds Expansion Wells

Bldg. Number: Not applicable

Map: Figures A-9, A-11, and A-15

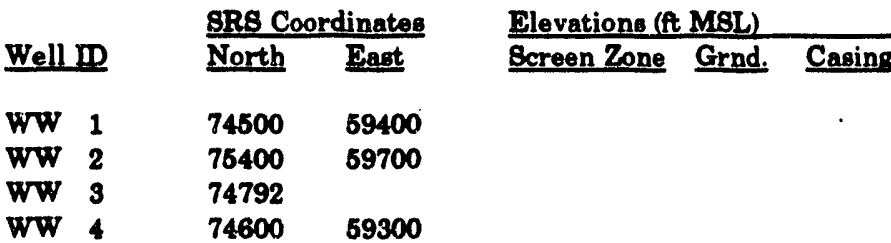

\section{XSB WELL SERIES}

Description: Old TNX Seepage Basin

Bldg. Number: 904-76T

Map: Figure A-29

\begin{tabular}{llll} 
& & \multicolumn{2}{l}{ gRS Coordinates } \\
\cline { 3 - 4 } Well ID & & North & East \\
\hline XSB 1 & & 71133.1 & 16901.0 \\
X8B & 1A & 71105.4 & 16883.0 \\
XSB 1B & 71105.0 & 16872.9 \\
XSB 1D & 71104.8 & 16893.5 \\
X8B & 2 & 71019.7 & 16948.0 \\
XSB & 2D & 71086.0 & 16823.1 \\
X8B & 3 & 70946.5 & 1689.9 \\
XSB & 3A & 70915.3 & 16901.3 \\
XSB & 4 & 71024.5 & 16851.1 \\
X8B & 4D & 70997.9 & 16826.2 \\
X8B & 5 & 70956.4 & 16703.8 \\
X8B & 5A & 70956.3 & 16703.7
\end{tabular}

\begin{tabular}{rrr}
\multicolumn{3}{c}{ Elevations (tt MSL) } \\
\cline { 1 - 2 } Bcreen Zone & Grnd. & Casing \\
\cline { 1 - 2 } & & \\
112.0 .92 .0 & 153.4 & 155.0 \\
$63.6-43.6$ & 153.6 & 156.0 \\
$74.6-64.6$ & 153.6 & 155.9 \\
107.9 .87 .9 & 153.9 & 156.0 \\
113.5 .93 .5 & 152.1 & 154.1 \\
104.0 .84 .0 & 153.0 & 154.8 \\
127.0 .107 .0 & 153.9 & 156.2 \\
& 154.3 & 157.0 \\
114.3 .94 .3 & 152.7 & 153.6 \\
103.9 .83 .9 & 152.9 & 154.9 \\
& 108.9 & 112.0 \\
& 108.9 & 112.0
\end{tabular}

(series continued)
Eff. Well

Depth(f) Caging Installed Abandoned

$$
\begin{aligned}
& 3^{n} \mathrm{Al} \\
& 3^{n} \mathrm{Al} \\
& 3^{n} \mathrm{Al}
\end{aligned}
$$

Al

$02-18-88$
$02-19-88$
$02-19-88$
$02-02-88$

Eff. Well

Depth (ft) Casing Installed Abardoned

$\begin{array}{rll}63.0 & 4^{n} \text { Steel } & 04-28-80 \\ 112.4 & 4 * & 11-21-89 \\ 91.3 & 4^{n} \text { PVC } & 11-22-89 \\ 68.1 & 4^{n} \text { PVC } & 09-09-88 \\ 60.6 & 4^{n} \text { Steel } & 04-30-80 \\ 70.8 & 4^{n} \text { PVC } & 09-12-88 \\ 49.2 & 4^{\prime \prime} \text { Stoel } & 04-14-80 \\ & \text { PVC } & 07-01-84^{*} \\ 69.2 & 4^{n} \text { Steel } & 04-16-80 \\ 71.0 & 4^{n} \text { PVC } & 09-12-88 \\ & 4^{\prime \prime} \text { PVC } & 09-12-88 \\ & \text { PVC } & \end{array}$

08-20-88

$09-01-88$

07.01.84*

09-01-88 
XSB WELL SERIES (cont.)

Notas: X8B aA has been identified as X8B ST in the past.

According to the Request for Surveying (10.17.81), on 8-24-81, $25.5 \mathrm{ft}$ of casing were added to X8B 4.

X8B 6 has been incorrectly identified as X8B $5 B$ in the past.

** XgB 1A has 4-in. PVC casing ingide 12-in. PVC casing. The ocreen is PVC.

\section{YSB WELL SERIES}

Description: New TNX Seepage Basin

Bldg. Number: 904-102T

Map: Figures A-16 and A-29

\begin{tabular}{|c|c|c|c|c|c|c|c|c|c|}
\hline \multirow[b]{2}{*}{ Well ID } & \multicolumn{2}{|c|}{ SRS Coordinateg } & \multicolumn{3}{|c|}{ Plevations (A MSL) } & \multirow{2}{*}{$\begin{array}{l}\text { Bff. Well } \\
\text { Depth (t) }\end{array}$} & \multirow[b]{2}{*}{ Caging } & \multirow[b]{2}{*}{ Installed } & \multirow[b]{2}{*}{ Abandonec } \\
\hline & North & Enet & Screen 7ore & Grnd. & Casing & & & & \\
\hline Y8B 1 & 71160.6 & 17798.6 & 135.7-116.7 & 143.0 & 146.0 & 29.3 & 4" Btoel & $04-08-80$ & $01-01.84^{*}$ \\
\hline YBB IA & 71162.2 & 17808.8 & $128.4-98.4$ & 149.4 & 146.5 & 47.1 & & $12-12-83$ & \\
\hline $\begin{array}{ll}\text { YBB } & 2 \\
\text { YBB } & 2 A\end{array}$ & $\begin{array}{l}71008.3 \\
71010.0\end{array}$ & $\begin{array}{l}17862.6 \\
17850.2\end{array}$ & $\begin{array}{l}138.0-118.0 \\
127.7-97.7\end{array}$ & $\begin{array}{l}146.1 \\
142.7\end{array}$ & $\begin{array}{l}147.1 \\
144.7\end{array}$ & $\begin{array}{l}29.1 \\
47.0\end{array}$ & $\begin{array}{l}\text { 4" Btoel } \\
\text { 4" PVC }\end{array}$ & $\begin{array}{l}04-09-80 \\
12-19-83\end{array}$ & $04-01.84 *$ \\
\hline YSB 3 & 70856.3 & 17764.8 & $135.6-116.6$ & 141.8 & 149.8 & 28.3 & 4" Btoel & $04-09-80$ & $01-01-84^{*}$ \\
\hline YSB SA & 70859.0 & 17765.2 & 126.7. 96.7 & 141.7 & 149.9 & 47.2 & 4" PVC & $12-28-83$ & \\
\hline $\begin{array}{ll}\text { YSB } & 4 \\
\text { YSB } & 4 A\end{array}$ & $\begin{array}{l}71050.8 \\
71020.7\end{array}$ & $\begin{array}{l}17742.3 \\
17799.8\end{array}$ & $\begin{array}{l}138.7-118.7 \\
127.6-97.6\end{array}$ & $\begin{array}{l}142.4 \\
142.6\end{array}$ & $\begin{array}{l}144.4 \\
144.6\end{array}$ & $\begin{array}{l}25.7 \\
47.0\end{array}$ & $\begin{array}{l}\text { Bteol } \\
\text { 4" PVC }\end{array}$ & $\begin{array}{l}04-10-80 \\
12-27-83\end{array}$ & $01-01-84^{*}$ \\
\hline
\end{tabular}

\section{YSC WELL SERIES}

Description: Y-Area Waste Solidification and Disposal Facility Bldg. Number: Not applicable

Map: Figure A-16

\begin{tabular}{|c|c|c|c|c|c|c|c|c|}
\hline \multirow[b]{2}{*}{ Well ID } & \multicolumn{2}{|c|}{ ERS Coordinates } & \multicolumn{3}{|c|}{ Plevations (A MSL) } & \multirow{2}{*}{$\begin{array}{l}\text { Dff. Well } \\
\text { Depth (f) }\end{array}$} & \multirow[b]{2}{*}{ Caging } & \multirow[b]{2}{*}{ Installed } \\
\hline & North & Pagt & Sereen Tone & Grnd. & Casing & & & \\
\hline $\mathbf{S C} \quad 1 \mathrm{~A}$ & 78039.9 & 65438.9 & 136.9- 76.8 & 268.9 & 270.9 & 194.1 & $4^{n \prime} \mathrm{P}$ & $11-10-89$ \\
\hline $1 \mathrm{C}$ & 78186.2 & 65856.6 & 207.5-197.5 & 272.6 & 274.4 & 76.9 & & $08-08-89$ \\
\hline 10 & 78170.7 & 65869.1 & 236.8-216.8 & 272.8 & 274.4 & 67.6 & & $06-02-89$ \\
\hline $8 C 2 A$ & 78311.6 & 66100.1 & 144.7.134.7 & 281.7 & 283.7 & 149.0 & & $06-30-89$ \\
\hline TSC 2D & 78320.4 & 66130.7 & 218.0-197.9 & 282.0 & 284.0 & 86.1 & & $06-30-89$ \\
\hline YEC $4 A$ & 77060.1 & 65888.6 & $131.0-121.0$ & 287.6 & 290.2 & 169.2 & & $08-12-89$ \\
\hline YBC $4 C$ & 77059.7 & 65901.9 & 205.9-195.9 & 287.9 & 289.6 & 93.7 & & $06-29-89$ \\
\hline TSC $6 A$ & 74295.9 & 67134.9 & $121.0-116.0$ & 273.0 & 274.9 & 168.9 & 4" PVC & 06-05-89 \\
\hline
\end{tabular}

Notes: YsC $1 \mathrm{~A}$ was first installed 6-14-89, but according to a Well Abandonment Report, this first well was officially abandoned 6-30-89 because of a "2-4 $\mathrm{ft}$ obetruction at about $105 \mathrm{ft}$ ineide well casing which hung up tag bar and was later drilled out .... Could not verify integrity of well casing." A second well by the same name was installed 6-30-89. According to page 1 of the Daily Activities Report, 11-06-89, for the third and final installation of Y8C 1A, the second well was unofficially abandoned when an obotruction was found at about $26 \mathrm{ft}$.

YEC 10 and $4 A$ are monitoring wells without pump 


\section{Z Well}

Description: Unofficial well in Z Area

Bldg. Number: Not applicable

Map: Not applicable

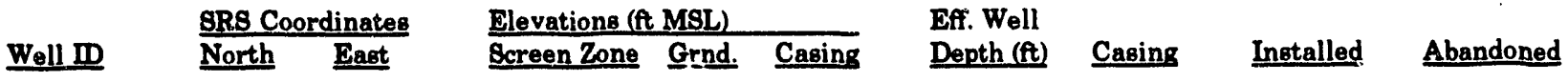

$\mathbf{Z}$

05-29-87

Notes: The identification of this well comes from the abandonment sheets. The $\mathrm{Z}$ designation is unofficial. The Well Abandonment Report describes this well as an unidentified, 4-t-equare, 40-ft-deep hand-dug well that was filled with grout as a safety precaution. This open hole was located near well ZBBG 2.

\section{Z WELL SERIES}

Description: F Area and $\mathrm{H}$ Area

Bldg. Number: Not applicable

Map: Figures A-2, A-12, A-13, A-14, and A-15

\begin{tabular}{|c|c|c|c|c|c|c|c|c|c|c|}
\hline \multirow{2}{*}{\multicolumn{2}{|c|}{ Well D }} & \multicolumn{2}{|c|}{ BRS Coordinates } & \multicolumn{3}{|c|}{ Plevations (A MBL) } & \multirow{2}{*}{$\begin{array}{l}\text { Bff. Well } \\
\text { Depth (ft) }\end{array}$} & \multirow[b]{2}{*}{ Casing } & \multirow[b]{2}{*}{ Installed } & \multirow[b]{2}{*}{ Abandoned } \\
\hline & & North & East & Screen Zone & Grnd. & Casing & & & & \\
\hline $\mathbf{Z}$ & 1 & 76220.5 & 52961.9 & $218.0-217.5$ & 279.7 & 281.2 & 63.7 & Bteel & $05-24-51$ & \\
\hline $\mathbf{z}$ & 2 & 74786.3 & 53181.6 & $214.6-214.0$ & 255.6 & 257.3 & 43.9 & Steel & $05-25-51$ & \\
\hline $\mathbf{Z}$ & 3 & 75088.2 & 51328.3 & 207.1-206.6 & 269.1 & 261.0 & 64.4 & Steel & $05-28-61$ & \\
\hline$\overline{\mathbf{z}}$ & 4 & 79440.0 & 51357.0 & 213.4-212.9 & 278.0 & 279.7 & 66.8 & 4" Btreel & $05-29-61$ & $05-02-61$ \\
\hline $\mathbf{Z}$ & b & 78145.0 & 61858.0 & 216.8-216.3 & 301.0 & 903.8 & 87.6 & Steel & $04-01-61 *$ & $06-06-76$ \\
\hline $\mathbf{z}$ & 6 & 78146.0 & 51858.0 & $225.2-205.2$ & 301.0 & 803.8 & 98.6 & $4^{n}$ Streel & $05-06-76$ & \\
\hline $\mathbf{z}$ & 6 & 76578.0 & 52989.0 & 221.8-221.3 & 283.8 & 285.6 & 64.3 & Steel & 0\&-01-61* & $05-02-61$ \\
\hline$\overline{\mathbf{Z}}$ & 7 & 77269.0 & 52166.0 & 215.9-215.4 & 289.1 & 291.0 & 75.6 & Steel & $06-07-51$ & 05.07 .76 \\
\hline $\mathbf{Z}$ & 7 & 77269.0 & 52156.0 & $224.4-204.4$ & 289.1 & 291.0 & 86.6 & 4" Btoel & $05-07-76$ & \\
\hline $\mathbf{z}$ & 8 & 76640.5 & 51684.9 & $214.1-213.6$ & 277.6 & 280.0 & 66.4 & Bteel & $06-07-61$ & \\
\hline $\mathbf{z}$ & 9 & 77732.0 & 50570.5 & 208.6-208.1 & 277.4 & 279.6 & 71.4 & Btoel & $04-01-61^{*}$ & $08-25.76$ \\
\hline $\mathbf{z}$ & 9 & 77732.0 & 50570.5 & $228.4-208.4$ & 277.4 & 279.5 & 71.1 & $4^{n}$ Steol & $06-25.76$ & \\
\hline $\bar{z}$ & 10 & 71999.0 & 60999.0 & $260.8-260.3$ & 282.3 & 289.7 & 29.4 & Bteel & $10-26-51$ & $01-03-68$ \\
\hline $\mathbf{Z}$ & 11 & 72639.5 & 61760.3 & 256.6-256.1 & 298.3 & 298.3 & 42.2 & Stoel & $10-01-61 *$ & \\
\hline $\mathbf{z}$ & 12 & 71198.9 & 61400.9 & $251.4-260.9$ & 293.6 & 295.6 & 44.7 & $0.75^{n}$ Steel & $10-29-51$ & $11-29-80$ \\
\hline $\bar{z}$ & 13 & 70785.8 & 62203.6 & 257.1-256.6 & 300.6 & 303.1 & 46.6 & Steel & $06-2 K-63$ & \\
\hline $\mathbf{z}$ & 14 & 71201.0 & 63000.0 & 262.6-262.1 & 297.8 & 300.4 & 38.3 & Steel & $10-23-61$ & $10-01-80 *$ \\
\hline $\mathbf{z}$ & 16 & 72802.1 & 63419.2 & $254.3-253.8$ & 305.1 & 306.9 & 63.1 & Steol & $10-01-61$ & \\
\hline $\mathbf{z}$ & 16 & 71379.0 & 63091.0 & $260.1-259.6$ & 299.3 & 302.0 & 42.4 & Steel & $10-01-61^{*}$ & $08-10-60$ \\
\hline $\mathbf{Z}$ & 17 & 72260.9 & 43797.8 & 148.7-148.2 & 176.2 & 178.8 & 30.6 & Stoel & $04-01-52$ & \\
\hline $\mathbf{z}$ & 18 & 73077.2 & 43774.1 & $160.4-169.9$ & 198.9 & 202.1 & 42.2 & Stoel & $04-03-62$ & \\
\hline $\mathbf{z}$ & 19 & 74726.0 & 43690.0 & $179.9-179.4$ & 261.7 & 263.8 & 84.4 & Steel & $04-04-62$ & $03-12-76$ \\
\hline $\mathbf{Z}$ & 19 & 74728.0 & 43690.0 & & 261.7 & 263.8 & & Steel & $06-23-76$ & \\
\hline $\mathbf{Z}$ & $19 A$ & 74716.1 & 49698.6 & $200.1-180.1$ & 260.1 & 262.0 & 81.9 & $4^{\prime \prime}$ Bteel & $06-23-76$ & \\
\hline $\mathbf{Z}$ & 20 & 74080.7 & 43722.4 & $174.3-173.8$ & 239.4 & 241.4 & 67.6 & Steel & $05-09-52$ & $06-24-76$ \\
\hline $\mathbf{z}$ & 20 & 74080.7 & 43722.4 & & 239.4 & 241.4 & & Bteel & $06-24-76$ & \\
\hline $\mathbf{Z}$ & $20 \mathrm{~A}$ & 74083.0 & 43719.0 & $104.8-104.3$ & 241.0 & 243.8 & 139.5 & Stoel & $05-09-62$ & $05-02-61$ \\
\hline $\mathbf{Z}$ & 20B & 74085.0 & 43721.0 & $195.6-175.6$ & 241.1 & 243.6 & 68.0 & 4" Btrel & $08-24-76$ & \\
\hline
\end{tabular}

(eeries continued) 


\title{
Z WELL SERIES (cont.)
}

Notes: Z 6, 7, 9, 19, and 20 were reworked in 1976. The 1976 abandonment dates and installation dates for these wells represent the dates the welle were reworked.

\begin{abstract}
Although no official abandonment date is available for $Z \mathbf{Z}$, the well was destroyed before 6-18-87 according to a note in the sampling echodule chain-of-custody reports.

The date (6-25-53) given for the installation of $\mathrm{Z} 13$ is for a new installation in an old boring. The original borehole was deepened. Data for the well indicate it was originally installed in 1951.
\end{abstract}

\section{ZBG WELL SERIES}

Description: Z-Area Saltstone Facility Background Wells

Bldg. INumber: Not applicable

Map: Figure A-16

\begin{tabular}{|c|c|c|c|c|c|c|c|c|c|}
\hline & BRs Cor & dinates & Blovations ( $f$ & MSL) & & Eff. Well & & & \\
\hline Well D & North & Pagt & Screen Zone & Grnd. & Caning & Depth (t) & Casing & Ingtalled & Abandoned \\
\hline $\begin{array}{ll}\text { ZABG } 1 \\
\text { ZABG } 1 A \\
\text { ZBG } 2\end{array}$ & $\begin{array}{l}76584.2 \\
76588.5 \\
76170.6\end{array}$ & $\begin{array}{l}65584.1 \\
65598.8 \\
67472.9\end{array}$ & $\begin{array}{l}240.1-220.0 \\
281.0-276.0 \\
290.9-210.9\end{array}$ & $\begin{array}{l}288.9 \\
287.8 \\
275.8\end{array}$ & $\begin{array}{l}291.1 \\
291.0 \\
278.0\end{array}$ & $\begin{array}{l}71.1 \\
16.0 \\
67.1\end{array}$ & $\begin{array}{l}4^{\prime \prime} \text { PVC } \\
4^{\prime \prime} \text { PVC } \\
4^{\prime \prime} \text { PVC }\end{array}$ & $\begin{array}{l}04-17-87 \\
06-01-87 \\
04-23-87\end{array}$ & \\
\hline
\end{tabular}

Notea: $A B G$ IA has been referred to as $2 A B G$ IP in the past.

\section{ZDT WELL SERIES}

Description: Z-Area Low Point Drain Tank

Bldg. Number: 551-Z

Map: Figure A-16

\begin{tabular}{|c|c|c|c|c|c|c|c|c|}
\hline \multirow[b]{2}{*}{ Well ID } & \multicolumn{2}{|c|}{ SRS Coondinates } & \multicolumn{3}{|c|}{ Elevations (A MSL) } & \multirow{2}{*}{$\begin{array}{l}\text { Eff. Well } \\
\text { Depth (A) }\end{array}$} & \multirow[b]{2}{*}{ Caping } & \multirow[b]{2}{*}{ Installed } \\
\hline & North & East: & Screen Zope & Grnd. & Casing & & & \\
\hline $\begin{array}{l}\text { ZADT } 1 \\
\text { ZADT } 12\end{array}$ & $\begin{array}{l}71644 . \\
71696 .\end{array}$ & $\begin{array}{l}65114.8 \\
65059.9\end{array}$ & $\begin{array}{l}247.0-227.0 \\
245.1-225.1\end{array}$ & $\begin{array}{l}263.0 \\
263.1\end{array}$ & $\begin{array}{l}265.1 \\
265.0\end{array}$ & $\begin{array}{l}38.1 \\
39.9\end{array}$ & $\begin{array}{l}\text { 4" PVC }^{\prime \prime} \\
4^{\prime \prime} \text { PVC }\end{array}$ & $\begin{array}{l}11-03-87 \\
11-04-87\end{array}$ \\
\hline
\end{tabular}

\section{ZW WELL SERIES}

Description: F Area and $\mathrm{H}$ Area

Bldg. Number: Not applicable

Map: Figures A-2, A-10, A-12, and A-14

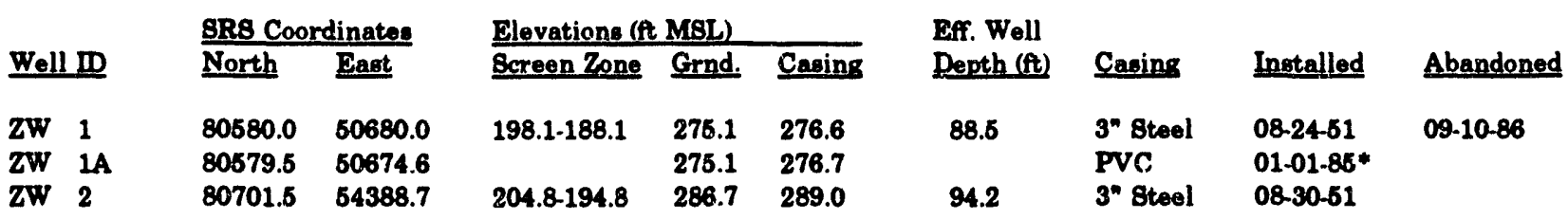

(series continued) 
ZW WELL SERIES (cont.)

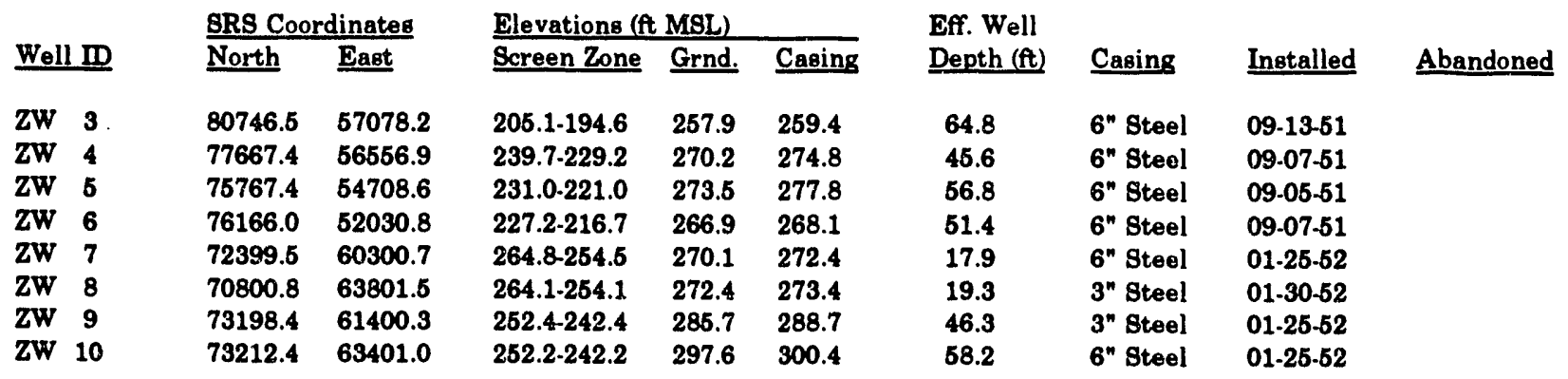

\section{1-F WELL SERIES}

Description: F-Area Tank Farm

Bldg. Number: 241-F

Map: Not applicable

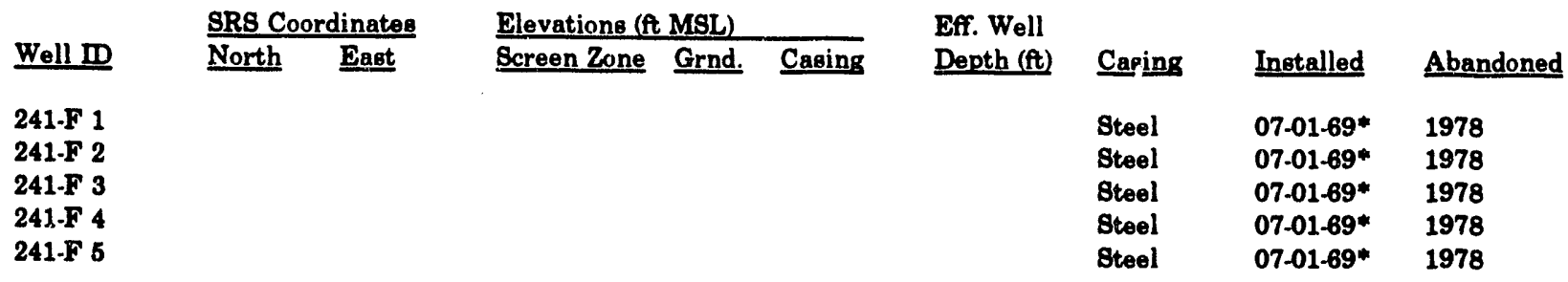

\section{1-H WELL SERIES}

Description: H-Area Tank Farm, between Tanks 9 and 11

Bldg. Number: 241-H

Map: Figure A-14

\begin{tabular}{|c|c|c|c|c|c|c|c|c|}
\hline & SRS Co & dinates & Flevations (ft MSL) & & Eff. Well & & & \\
\hline Well ID & North & East & Screen Zone Grnd. & Casing & Depth (ft) & Casing & Installed & Abandoned \\
\hline 241-H & 71639.9 & 62034.6 & & 283.4 & & Steel & $07-01-59^{*}$ & \\
\hline
\end{tabular}

700-A WELL (See ABG WELL SERIES, ABW WELL SERIES) 
This page was left blank intentionally. 


\section{BIBLIOGRAPHY}

Ashley, C., and C. C. Zeigler. (1975). Environmental Monitoring at the Savannah River Plant: Annual Report-1974, DPSPU 75-302. Aiken, SC: E. I. du Pont de Nemours and Company, Health Physics Department, Savannah River Plant.

Ashley, C., and C. C. Zeigler. (1978). Environmental Monitoring at the Savannah River Plant: Annual Report-1976, DPSPU 77-302. Aiken, SC: E. I. du Pont de Nemours and Company, Health Physics Department, Savannah River Plant.

Ashley, C., P. C. Padezanin, and C. C. Zeigler. (1984a). Environmental Monitoring at the Savannah River Plant: Annual Report-1982, DPSPU 83-302. Aiken, SC: E. I. du Pont de Nemours and Company, Health Protection Department, Savannah River Plant.

Ashley, C., P. C. Padezanin, and C. C. Zeigler. (1984b). Environmental Monitoring at the Savannah River Plant: Annual Report-1983, DPSPU 84-302. Aiken, SC: E. I. du Pont de Nemours and Company, Health Protection Department, Savannah River Plant.

Christensen, E. J., and D. E. Gordon, eds. (1983). Technical Summary of Groundwater Quality Protection Program at Savannah River Plant, Site Geohydrology and Solid and Hazardous Wastes, DPST-83-829 (Vol. I). Aiken, SC: E. I. du Pont de Nemours and Company, Savannah River Laboratory.

Clark, W. E. (n.d.). Ground-Water Flow Paths and Rates In and Near H Area, Savannah River Plant, South Carolina. Prepared in cooperation with the U.S. Atomic Energy Commission.

D'Appolonia Consulting Engineers. (n.d.). Report: Boring and Instrument Installation DWPF Salt Disposal Site.

Environmental Monitoring (Health Protection Department) and Exploration Software. (1988). The Savannah River Plant's Groundwater Monitoring Program Conducted by the Heait, Protection Department for the Second Quarter 1988, HPR-88300. Aiken, SC: E. I. du Pont de Nemours and Company, Savannah River Plant.

Fenimore, J. W., and J. H. Horton. (1972). Operating History and Environmental Effects of Seepage Basins in Chemical Separations Areas of the Savannah River Plant, DPST-72-548. Aiken, SC: E. I. du Pont de Nemours and Company, Savannah River Laboratory. 
Geraghty \& Miller, Inc. (1985). H-Area Seepage Basin Well Installation Data Report, Section IV, Monthly Summaries. Aiken, SC: E. I. du Pont de Nemours and Company, Savannah River Laboratory.

Geraghty \& Miller, Inc. (1986). Final Report: Installation of Monitor Wells in the $\mathbf{A} / \mathbf{M}$ Areas of the Savannah River Plant. Prepared for E. I. du Pont de Nemours \& Company, Inc., Savannah River Plant. G \& M project number, A315AM1. September 1985. Revised February 1986. Aiken, SC: Geraghty \& Miller, Inc.

Heckrotte, R. W. (1987). DWPF S-Area and Z-Area Soil Boring and Well Closure. Aiken, SC: E. I. du Pont de Nemours and Company, Interoffice Memorandum, Savannah River Laboratory.

Janssen, J., and Exploration Resources. (1990). Environmental Protection Well Inventory, Includes Current and Past Monitoring (Through the Fourth Quarter of 1989), ESH-EMS-900037. Aiken, SC: Westinghouse Savannah River Company, Savannah River Site.

Marine, I. W., and H. W. Bledsoe. (1985). Supplemental Technical Data Summary: MArea Groundwater Investigation, DPSTD-84-112. Aiken, SC: E. I. du Pont de Nemours and Company, Savannah River Laboratory.

Stone, J. A., and E. J. Christensen, eds. (1983). Technical Summary of Ground-Water Quality Protection Prosisam at Savannah River Plant, Radioactive Waste, DPST-83-829 (Vol. II). Aiken, SC: E. I. du Pont de Nemours and Company, Savanneh River Laboratory.

United States Department of Energy. (1986a). Application for a Post-Closure Permit, MArea Hazardous Waste Management Facility (Vol. III), Appendix B, Book 1, "Well Construction Records and Geologic Data." Revision No. 1, July 1986. Savannah River Plant.

United States Department of Energy. (1986b). Application for a Post-Closure Permit, MArea Hazardous Waste Management Facility (Vol. III), Appendix B, Brnk 2, "Well Construction Records and Geologic Data." Revision No. 1, July 1986. Savannah River Plant.

Works Technical Department. (1962). Health Physics Regional Monitoring Semiannual Report, January through June 1961, DPSP 62-25-2. Aiken, SC: E. I. du Pont de Nemours and Company, Explosives Department, Atomic Energy Division, Savannah River Plant.

Zeigler, C. C., I. B. Lawrimore, and W. E. O'Rear. (1985). Environmental Monitoring at the Savannah River Plant, Annual Report-1984, DPSPU 85-302. Aiken, SC: E. I. du Pont de Nemours and Company, 'Jealth Protection Department, Savannah River Plant. 


\section{BUILDING NUMBER INDEX}

Building or Facility Number

Well Series

080-17G, -17.1G, -18G, -18.1G, -18.2G, -18.3G, -19G CMP

105-C CDB

105-K KDB

105-L LDB

105-P PDB

105-R RDB RSD

$108-1 \mathrm{~K}$ KDT

131-C CRP

$131-\mathrm{K}$ KRP

131-L LRP

131-P PRP

131-R, -1R RRP

188-K $\mathrm{KAB}$

189-C $\mathrm{CCB}$

189-K $\mathrm{KCB}$

189-P PCB

221-F FCA

221-1F FAL

221-H HCA $\mathrm{HCC}$ 


\section{Building or Facility Number}

221-S

231-F, -1F

231-4F

241-F

$241-F,-6 F,-11 F,-14 F,-54 F$

241-H
Well Series

SCA

FBP

BRR

241-F

FTF

HP

HPM

TW

241-H

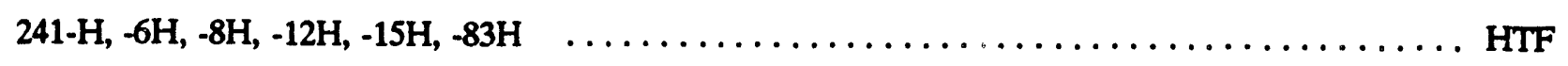

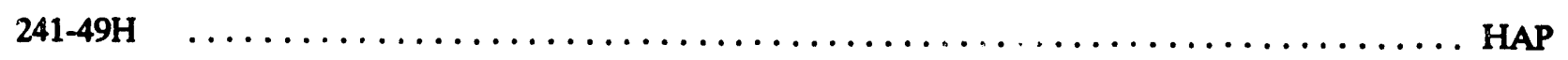

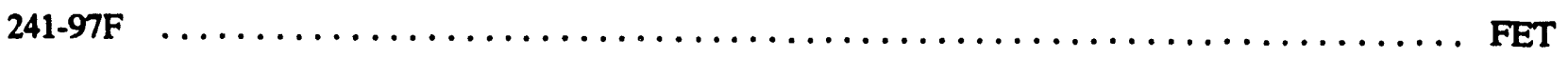

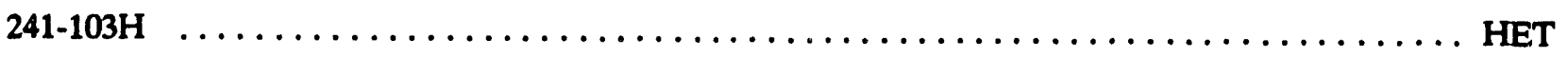

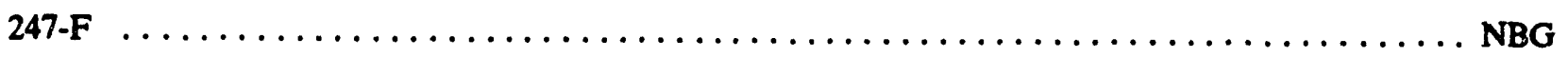

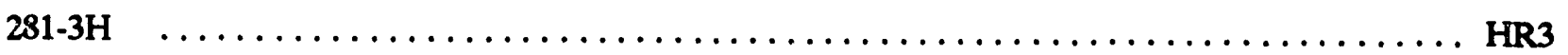

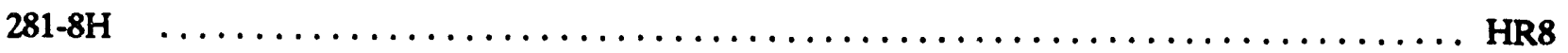

$289-\mathrm{F} \ldots \ldots \ldots \ldots \ldots \ldots \ldots \ldots \ldots \ldots \ldots \ldots \ldots \ldots \ldots \ldots \ldots \ldots \ldots \ldots \ldots$

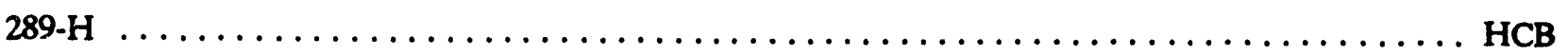

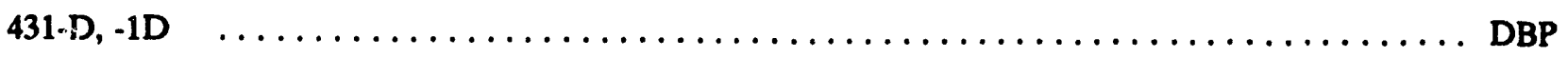

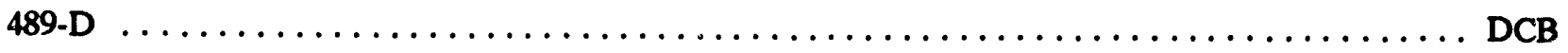

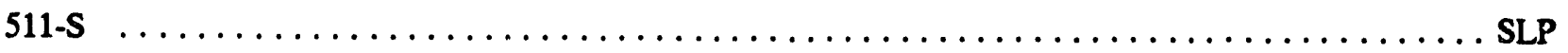

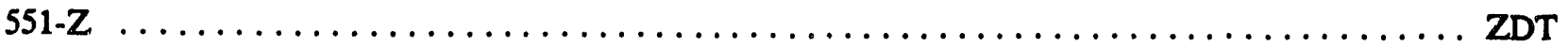

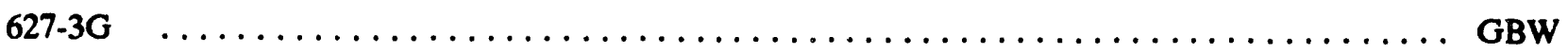

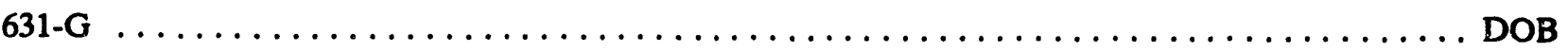




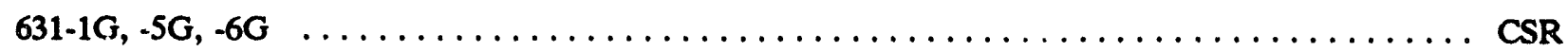

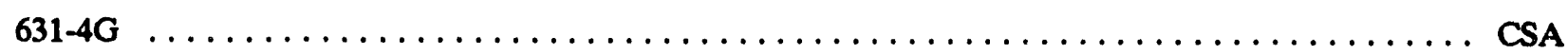

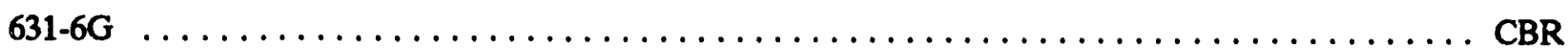

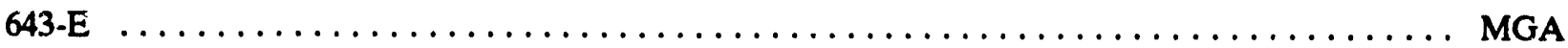

MGC

MGE

MGG

MGI

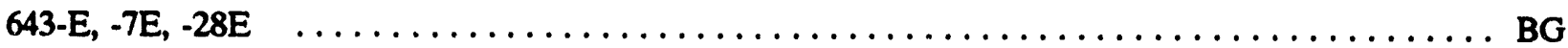

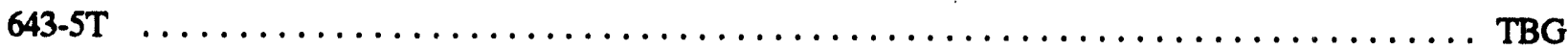

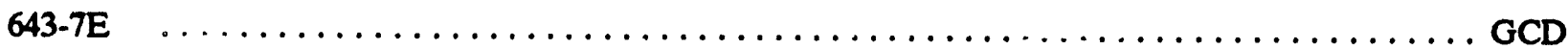

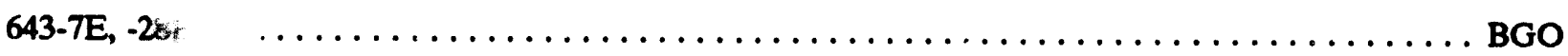

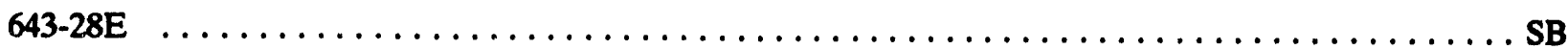

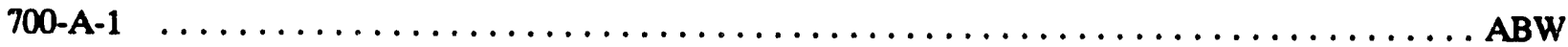

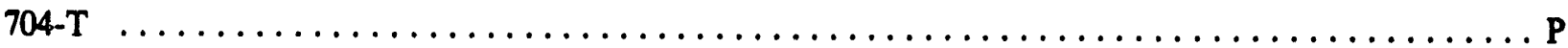

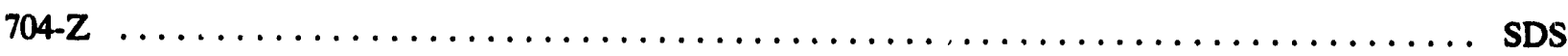

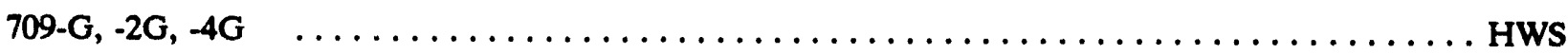

724-7G $\ldots \ldots \ldots \ldots \ldots \ldots \ldots \ldots \ldots \ldots \ldots \ldots \ldots \ldots \ldots \ldots \ldots \ldots \ldots \ldots \ldots \ldots \ldots$

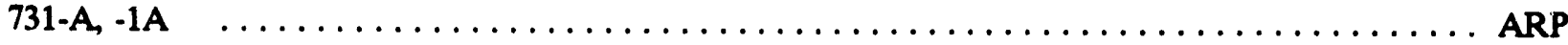

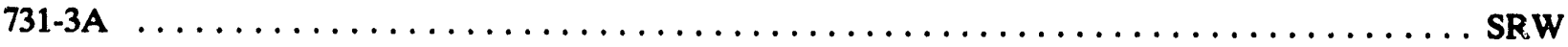

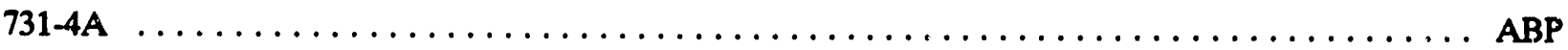

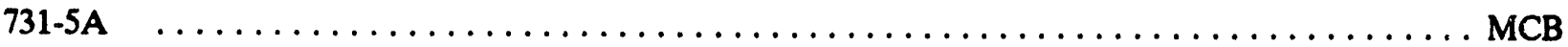

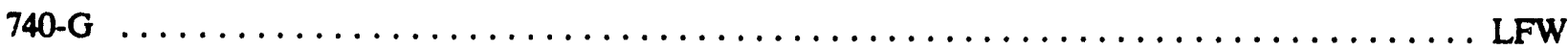

$761-G,-1 G,-2 G,-3 G,-4 G,-5 G,-6 G,-7 G,-8 G \quad \ldots \ldots \ldots \ldots \ldots \ldots \ldots \ldots \ldots \ldots \ldots \ldots$ sSS 
$761-5 G$

788-3.A ACB

8318 CSD

$904-41 G,-42 G,-43 G$ . F FSB

$904-44 G,-45 G,-46 G,-56 G$ H HS.

$904-49 G$ FNB

904-51G, -112G MSB

$904-53 G 1,-53 G 2,-54 G,-55 G$ ASB

904-56G BG

904-60G RSA

904-61G, $-62 G,-63 G$ PSB

904-64G LSB

904-65G KSB

$904-66 G,-67 G,-68 G$ CSB

904-74G FAC

904-74G HAC

904-76T XSB

904-77G RAC

904-78G PAC

904-79G LAC

904-80G $\mathrm{KAC}$

904-83G LCO 
KWL

904-91G HXB

904-101G AOB

904-102T YSB

904-103G RSB RSD

904-103G, -57G, -58G RSE

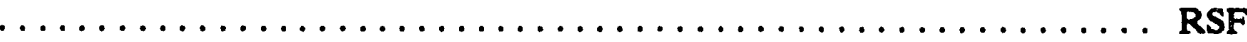

904-103G, -104G, -57G, -58G, -.59G RSW

904-103G, -104G, -57G, -58G, -59G, -6nG RSC

904-110G AMB

904-111G BRD

$904-112 \mathrm{G}$ CSO 
This page was left blank intentionally. 


\section{DESCRIPTION INDEX}

Description

Well Series

A-Area Background Well near Firing Range $\ldots \ldots \ldots \ldots \ldots \ldots \ldots \ldots \ldots \ldots \ldots \ldots \ldots$

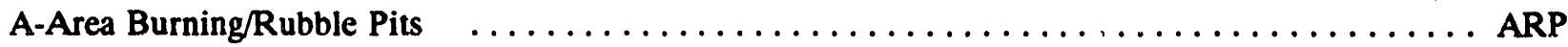

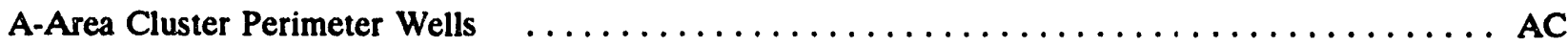

A-Area Coal Pile Runoff Containment Basin $\ldots \ldots \ldots \ldots \ldots \ldots \ldots \ldots \ldots \ldots \ldots \ldots \ldots$

A-Area Metals Burning Pit $\quad \ldots \ldots \ldots \ldots \ldots \ldots \ldots \ldots \ldots \ldots \ldots \ldots \ldots \ldots \ldots \ldots \ldots \ldots$

Aquifer Characterization Test Wells in $\mathrm{H}$ Area between
the Burial Grounds and Road $4 \ldots \ldots \ldots \ldots \ldots \ldots \ldots \ldots \ldots \ldots \ldots \ldots \ldots$

Background Well near Hawthorne Fire Tower $\ldots \ldots \ldots \ldots \ldots \ldots \ldots \ldots \ldots \ldots \ldots$ GBW

B-Area Microbio ogy Wells $\ldots \ldots \ldots \ldots \ldots \ldots \ldots \ldots \ldots \ldots \ldots \ldots \ldots \ldots \ldots$

Bentonite Test Pit Wells, across from the entrance

to the Burial Grounds on Road $\mathrm{E} \quad \ldots \ldots \ldots \ldots \ldots \ldots \ldots \ldots \ldots \ldots \ldots \ldots \ldots \ldots \ldots \ldots$

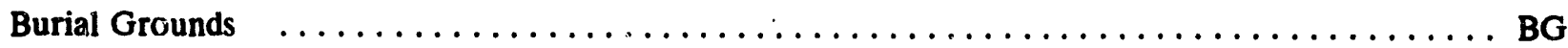

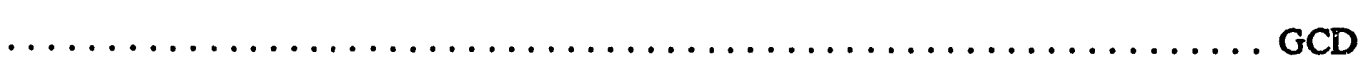

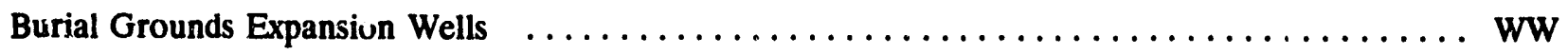

Burial Grounds Grid Wells $\quad \ldots \ldots \ldots \ldots \ldots \ldots \ldots \ldots \ldots \ldots \ldots \ldots \ldots \ldots \ldots \ldots \ldots$

Burial Grounds Perimeter Wells $\quad \ldots \ldots \ldots \ldots \ldots \ldots \ldots \ldots \ldots \ldots \ldots \ldots \ldots \ldots \ldots$

Burma Road Rubble Pit $\ldots \ldots \ldots \ldots \ldots \ldots \ldots \ldots \ldots \ldots \ldots \ldots \ldots \ldots \ldots \ldots$

C-Area Burning/Rubble Pit $\ldots \ldots \ldots \ldots \ldots \ldots \ldots \ldots \ldots \ldots \ldots \ldots \ldots \ldots \ldots$

C-Area Coal Pile Runoff Containment Basin $\ldots \ldots \ldots \ldots \ldots \ldots \ldots \ldots \ldots \ldots \ldots \ldots$

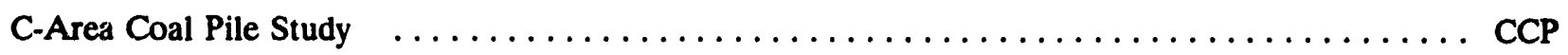

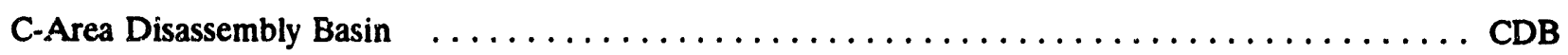


Description

Well Series

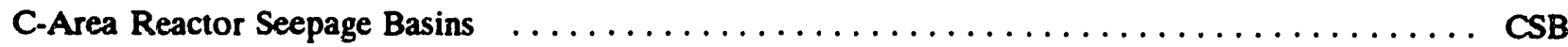

Central Shops Burning/Rubble Pit south of the Ford Building Seepage Basin $\ldots \ldots \ldots \ldots \ldots \ldots \ldots \ldots \ldots \ldots \ldots \ldots \ldots \ldots$

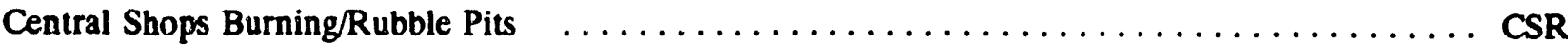

Central Shops Diesel Spill Characterization and Remediation Wells CSD

Characterization pie" ometers for the proposed Sanitary Landfill, Site B, off E2 Road $\ldots \ldots \ldots \ldots \ldots \ldots \ldots \ldots \ldots \ldots \ldots$ BTP

Chemicak, Metals, and Pesticides (CMP) Pits $\ldots \ldots \ldots \ldots \ldots \ldots \ldots \ldots \ldots \ldots \ldots$ CMP

D-Area Burning/Rubble Pits $\quad \ldots \ldots \ldots \ldots \ldots \ldots \ldots \ldots \ldots \ldots \ldots \ldots \ldots \ldots \ldots \ldots \ldots \ldots \ldots$

D-Area Coal Pile Runoff Containment Basin

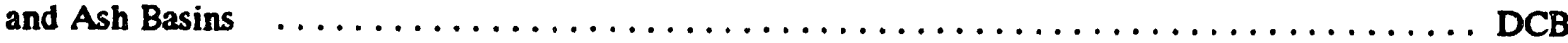

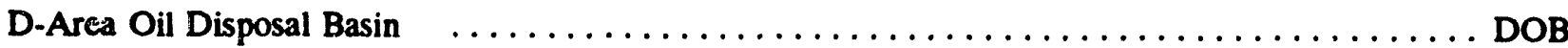

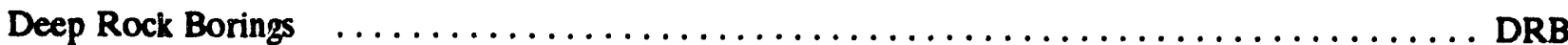

E-Area Vaults near the Burial Grounds $\ldots \ldots \ldots \ldots \ldots \ldots \ldots \ldots \ldots \ldots \ldots \ldots \ldots \ldots$

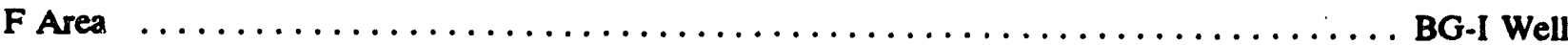

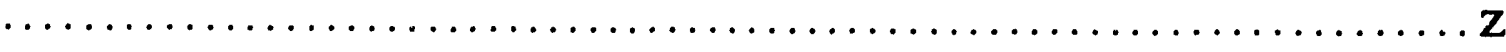
ZW

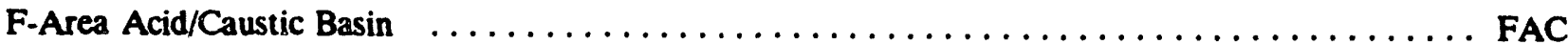

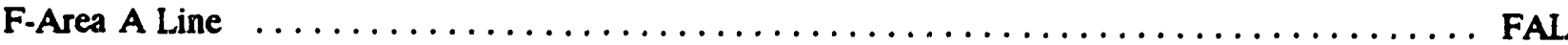

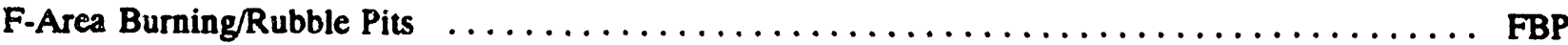

F-Area Canyon Building $\quad \ldots \ldots \ldots \ldots \ldots \ldots \ldots \ldots \ldots \ldots \ldots \ldots \ldots \ldots \ldots \ldots \ldots \ldots \ldots$

F-Area Coal Pile Runoff Containment Basin $\ldots \ldots \ldots \ldots \ldots \ldots \ldots \ldots \ldots \ldots \ldots$ FCB

F-Area Effluent Treatment Cooling Water Basin $\ldots \ldots \ldots \ldots \ldots \ldots \ldots \ldots \ldots \ldots \ldots$ FET

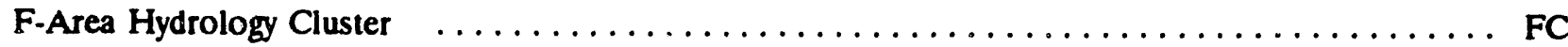

F-Area Microbiology Wells $\ldots \ldots \ldots \ldots \ldots \ldots \ldots \ldots \ldots \ldots \ldots \ldots \ldots \ldots \ldots \ldots \ldots$ 
Grid Wells

H Area

H-Area Well

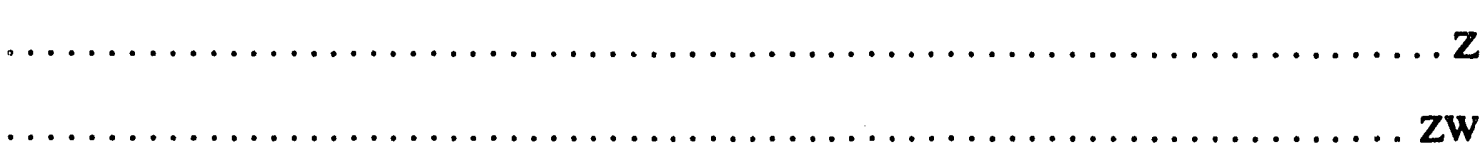

H-Area Acid/Caustic Basin HAC

H-Area Auxiliary Pump Pit HAP

H-Area Canyon Building HCA HCC

H-Area Coal Pile Runoff Containment Basin $\mathrm{HCB}$

H-Area Effluent Treatment Cooling Water Basin HET 
Description

Well Series

H-Area Hydrology Cluster $\ldots \ldots \ldots \ldots \ldots \ldots \ldots \ldots \ldots \ldots \ldots \ldots \ldots \ldots \ldots \ldots \ldots$

H-Area Retention Basin $\ldots \ldots \ldots \ldots \ldots \ldots \ldots \ldots \ldots \ldots \ldots \ldots \ldots \ldots \ldots \ldots$

H-Area Seepage Basins $\ldots \ldots \ldots \ldots \ldots \ldots \ldots \ldots \ldots \ldots \ldots \ldots \ldots \ldots \ldots \ldots$

HSB

H-Area Seepage Basins Production Weil $\ldots \ldots \ldots \ldots \ldots \ldots \ldots \ldots \ldots \ldots$ HSB 1TB Well

H-Area Sludge Land Application Site $\quad \ldots \ldots \ldots \ldots \ldots \ldots \ldots \ldots \ldots \ldots \ldots \ldots \ldots \ldots \ldots \ldots$

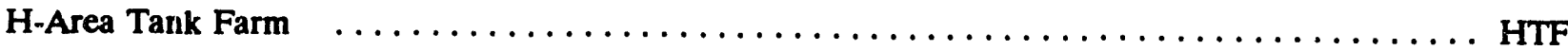

H-Area Tank Farm, between Tanks 9 and $11 \ldots \ldots \ldots \ldots \ldots \ldots \ldots \ldots \ldots \ldots \ldots$

Hazardous Waste/Mixed Waste Disposal Facility $\ldots \ldots \ldots \ldots \ldots \ldots \ldots \ldots \ldots \ldots \ldots \ldots$ HMD

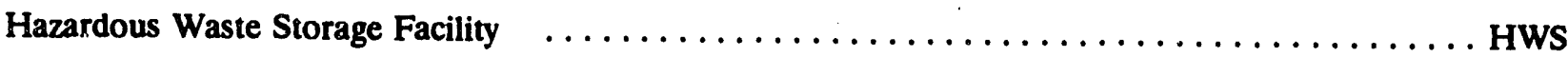

Hydrofluoric Acid Spill Area $\ldots \ldots \ldots \ldots \ldots \ldots \ldots \ldots \ldots \ldots \ldots \ldots \ldots \ldots \ldots \ldots \ldots \ldots$

Interim Waste Technology Site Characterization Wells,

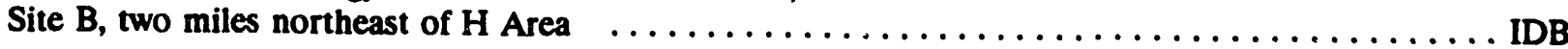

Interim Waste Technology Site Characterization Wells,

Site $P$, adjacent to Site $Q$, and located south of the

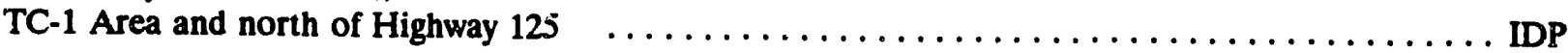

Interim Waste Technology Site Characterization Wells,

Site $Q$, adjacent to Site $P$, and located south of the

TC-1 Area and north of Highway $125 \quad \ldots \ldots \ldots \ldots \ldots \ldots \ldots \ldots \ldots \ldots \ldots \ldots \ldots$

Just north of the Burial Grounds $\ldots \ldots \ldots \ldots \ldots \ldots \ldots \ldots \ldots \ldots \ldots \ldots \ldots \ldots$

Just outside the fence surrounding the Burial Grounds;

BG 43,52 , and 67 are inside the fence $\ldots \ldots \ldots \ldots \ldots \ldots \ldots \ldots \ldots \ldots \ldots \ldots \ldots$

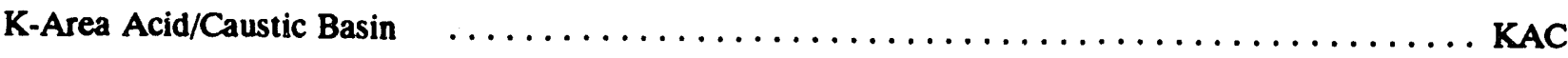

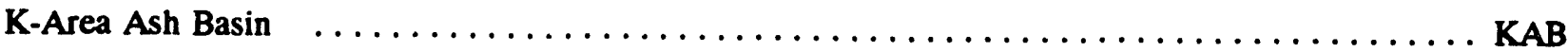

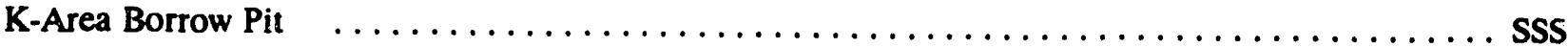

K-Area Burning/Rubble Pit $\ldots \ldots \ldots \ldots \ldots \ldots \ldots \ldots \ldots \ldots \ldots \ldots \ldots \ldots \ldots \ldots \ldots \ldots$

K-Area Coal Pile Runoff Containment Basin $\ldots \ldots \ldots \ldots \ldots \ldots \ldots \ldots \ldots \ldots \ldots \ldots$

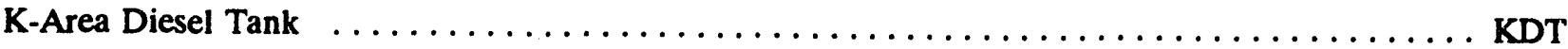


Description

Well Series

K-Area Disassembly Basin $\ldots \ldots \ldots \ldots \ldots \ldots \ldots \ldots \ldots \ldots \ldots \ldots \ldots \ldots \ldots, \ldots \ldots, \ldots \ldots$

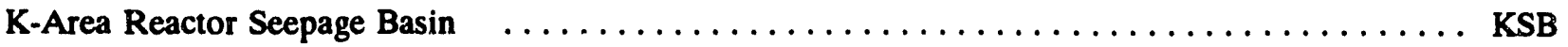

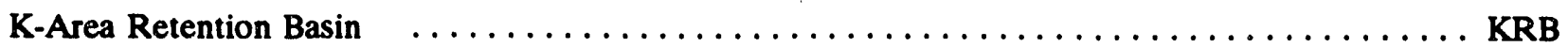

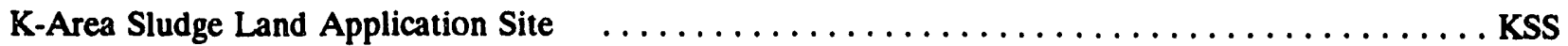

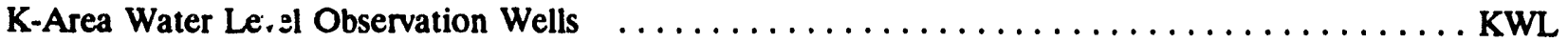

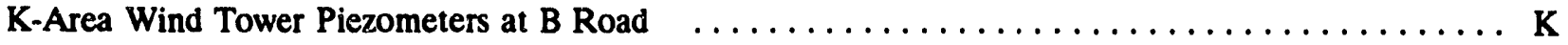

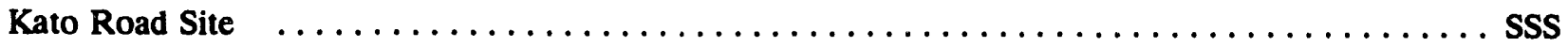

L-Area Acid/Caustic Basin $\ldots \ldots \ldots \ldots \ldots \ldots \ldots \ldots \ldots \ldots \ldots \ldots \ldots$ LAC

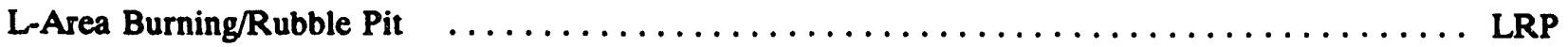

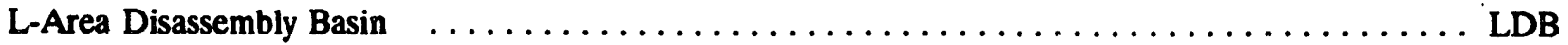

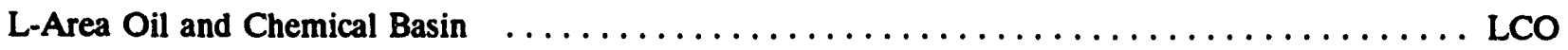

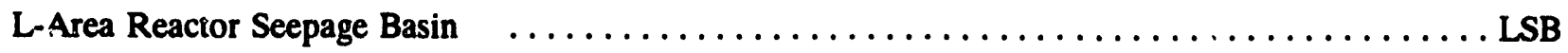

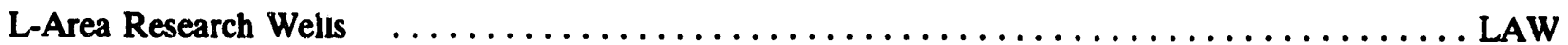

Lower Kato Road Site $\ldots \ldots \ldots \ldots \ldots \ldots \ldots \ldots \ldots \ldots \ldots \ldots \ldots \ldots \ldots$. sss

M-Area Hazardous Waste Management Facility (HWMF) $\ldots \ldots \ldots \ldots \ldots \ldots \ldots \ldots \ldots$ MSB

M-Area Plume Definition Wells $\ldots \ldots \ldots \ldots \ldots \ldots \ldots \ldots \ldots \ldots \ldots \ldots \ldots \ldots \ldots, \ldots \ldots \ldots$

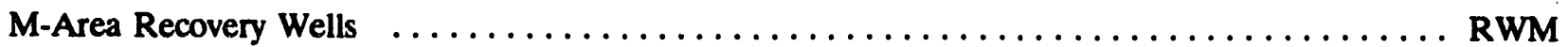

M-Area Settling Basin (also used for plume definition) $\ldots \ldots \ldots \ldots \ldots \ldots \ldots \ldots \ldots, \ldots \ldots$

M-Area Vadose Zone Extraction Wells $\ldots \ldots \ldots \ldots \ldots \ldots \ldots \ldots \ldots \ldots \ldots \ldots \ldots \ldots \ldots$ MVE

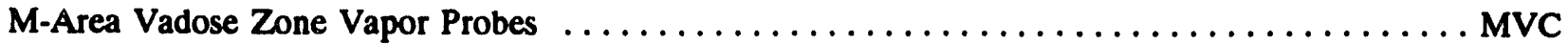

M-Area Wells Required by the SRS Hazardous Waste Permit $\ldots \ldots \ldots \ldots \ldots \ldots \ldots \ldots$ MSB

Metallurgical Laboratory Seepage Basin $\ldots \ldots \ldots \ldots \ldots \ldots \ldots \ldots \ldots \ldots \ldots \ldots \ldots$ AMB

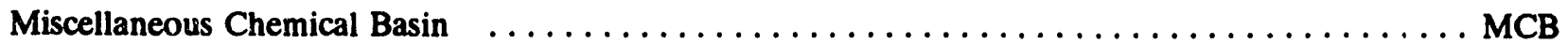

Mixed Waste Management Facility (MWMF) Borrow Pit $\ldots \ldots \ldots \ldots \ldots \ldots \ldots \ldots$ Kell

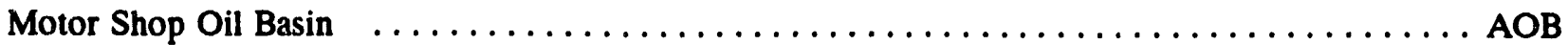




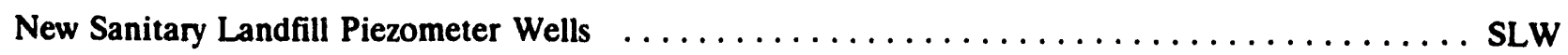

New TNX Seepage Basin $\ldots \ldots \ldots \ldots \ldots \ldots \ldots \ldots \ldots \ldots \ldots \ldots \ldots \ldots \ldots \ldots$

Old F-Area Seepage Basin $\ldots \ldots \ldots \ldots \ldots \ldots \ldots \ldots \ldots \ldots \ldots \ldots \ldots \ldots \ldots$

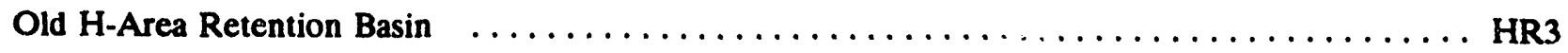

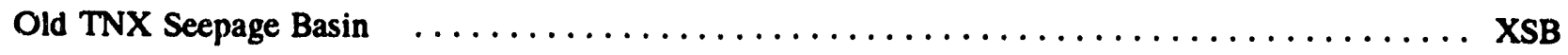

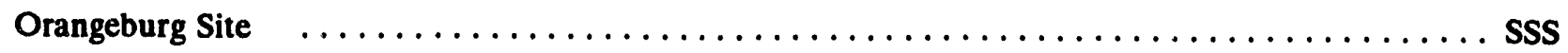

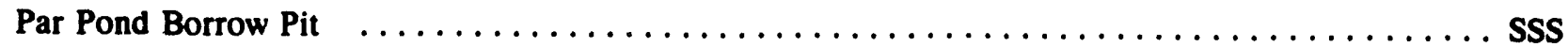

Par Pond Sludge Land Application Site $\ldots \ldots \ldots \ldots \ldots \ldots \ldots \ldots \ldots \ldots \ldots \ldots$

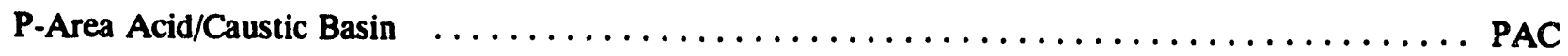

P-Area Burning/Rubble Pit $\quad \ldots \ldots \ldots \ldots \ldots \ldots \ldots \ldots \ldots \ldots \ldots \ldots \ldots \ldots \ldots \ldots$

P-Area Coal Pile Runoff Containment Basin $\ldots \ldots \ldots \ldots \ldots \ldots \ldots \ldots \ldots \ldots \ldots$

P-Area Disassembly Basin $\ldots \ldots \ldots \ldots \ldots \ldots \ldots \ldots \ldots \ldots \ldots \ldots \ldots \ldots \ldots$

P-Area Reactor Seepage Basins $\ldots \ldots \ldots \ldots \ldots \ldots \ldots \ldots \ldots \ldots \ldots \ldots \ldots \ldots \ldots \ldots$

Potential Hazardous Waste/Mixed Waste Disposal Facility $\ldots \ldots \ldots \ldots \ldots \ldots \ldots \ldots \ldots$ MWD

Preliminary Hydrogeologic Evaluation of Potential

New Production Reactor Site Characterization

Wells

NPM

NPN

NTN

NTS

NTW

Production Wells

DRB 5WW Well

FSB 1TA Well

HPT 1A Well

HSB 1TB Well

PW 
Description

Well Series

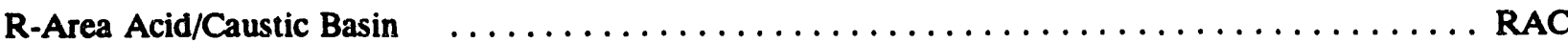

R-Area Burning/Rubble Pits $\ldots \ldots \ldots \ldots \ldots \ldots \ldots \ldots \ldots \ldots \ldots \ldots \ldots \ldots \ldots \ldots \ldots \ldots$

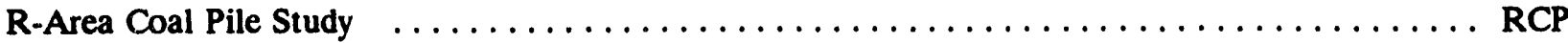

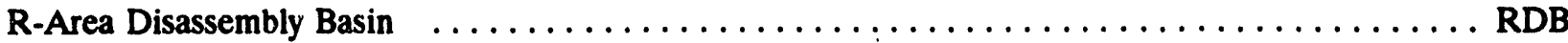

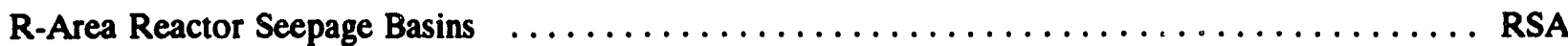

RSB

RSC

RSD

RSE

RSF

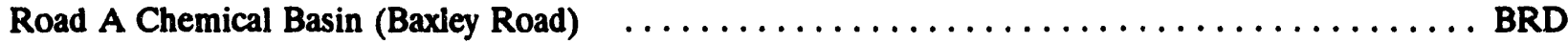

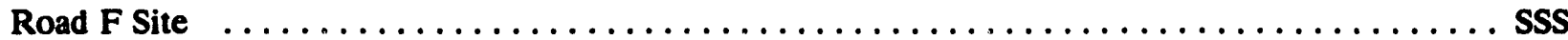

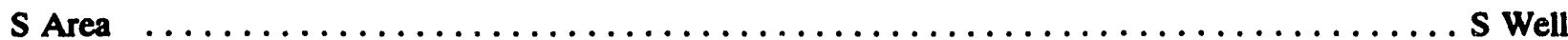

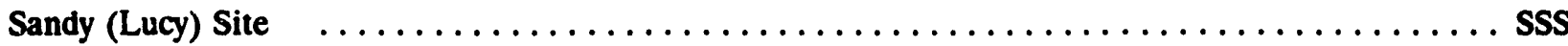

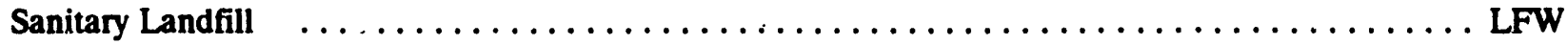

S-Area Defense Waste Processing Facility (DWPF) $\ldots \ldots \ldots \ldots \ldots \ldots \ldots \ldots \ldots \ldots$ RSS

S-Area Defense Waste Processing Facility (DWPF)

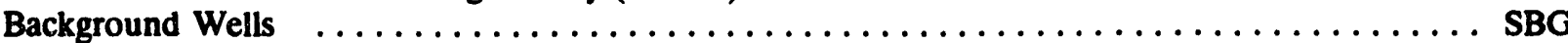

S-Area Defense Waste Processing Facility , DWPF)
Boreholes and Piezometers $\ldots \ldots \ldots \ldots \ldots \ldots \ldots \ldots \ldots \ldots \ldots \ldots \ldots \ldots, \ldots \ldots, \ldots \ldots$

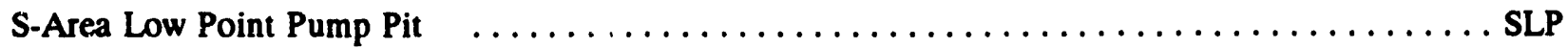

S-Area Vitrification Building $\ldots \ldots \ldots \ldots \ldots \ldots \ldots \ldots \ldots \ldots \ldots \ldots \ldots \ldots \ldots \ldots \ldots$

Savannah River Laboratory (SRL) Monitoring Wells for the
Horizontal Well Characterization Study in M Area $\ldots \ldots \ldots \ldots \ldots \ldots \ldots \ldots \ldots \ldots$ MHT

Savannah River Laboratory (SRL) Seepage Basins $\ldots \ldots \ldots \ldots \ldots \ldots \ldots \ldots \ldots \ldots \ldots \ldots$

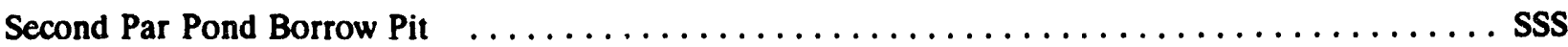


Description

Well Series

Series A, Monitoring Grid Wells for Burial Grounds $\ldots \ldots \ldots \ldots \ldots \ldots \ldots \ldots \ldots$ MGA

Series A, R-Area Reactor Seepage Basins $\quad \ldots \ldots \ldots \ldots \ldots \ldots \ldots \ldots \ldots \ldots \ldots \ldots$ RSA

Series B, R-Area Reactor Seepage Basins $\quad \ldots \ldots \ldots \ldots \ldots \ldots \ldots \ldots \ldots \ldots \ldots \ldots \ldots$ RSB

Series C, Monitoring Grid Wells for Burial Grounds $\ldots \ldots \ldots \ldots \ldots \ldots \ldots \ldots \ldots$

Series C, R-Area Reactor Seepage Basins $\quad \ldots \ldots \ldots \ldots \ldots \ldots \ldots \ldots \ldots \ldots \ldots \ldots \ldots$ RSC

Series D, between R-A.ea Reactor Seepage Basins

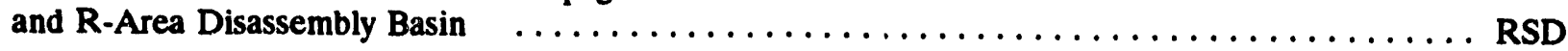

Series DW, R-Area Reactor Seepage Basins Dry

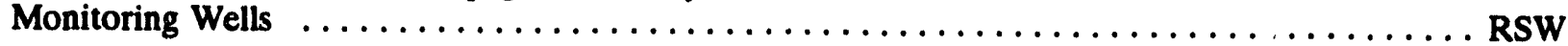

Series E, Monitoring Grid Wells for Burial Grounds $\ldots \ldots \ldots \ldots \ldots \ldots \ldots \ldots \ldots \ldots$

Series E, R-Area Reactor Seepage Basin; $\quad \ldots \ldots \ldots \ldots \ldots \ldots \ldots \ldots \ldots \ldots \ldots \ldots \ldots$

Series F, R-Area Reactor Seepage Basins $\quad \ldots \ldots \ldots \ldots \ldots \ldots \ldots \ldots \ldots \ldots \ldots \ldots \ldots$ RSF

Series $G$, Monitoring Grid Wells for Burial Grounds $\ldots \ldots \ldots \ldots \ldots \ldots \ldots \ldots$. $\ldots \ldots$

Series I, Monitoring Grid Welis for Burial Grounds $\ldots \ldots \ldots \ldots \ldots \ldots \ldots \ldots \ldots \ldots$ MGI

Sewage Sludge Application Sites $\quad \ldots \ldots \ldots \ldots \ldots \ldots \ldots \ldots \ldots \ldots \ldots \ldots \ldots \ldots \ldots$ sss

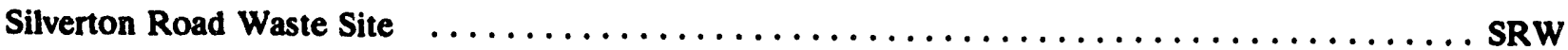

Special Burial Wells in the Burial Grounds $\ldots \ldots \ldots \ldots \ldots \ldots \ldots \ldots \ldots \ldots \ldots \ldots \ldots$

SRS Baseline Hydrogeologic Investigation Observation

Well Clusters $\ldots \ldots \ldots \ldots \ldots \ldots \ldots \ldots \ldots \ldots \ldots \ldots \ldots \ldots \ldots \ldots \ldots \ldots \ldots$

Swamp area southeast of $\mathrm{H}$-Area Seepage Basins $\ldots \ldots \ldots \ldots \ldots \ldots \ldots \ldots \ldots \ldots \ldots$

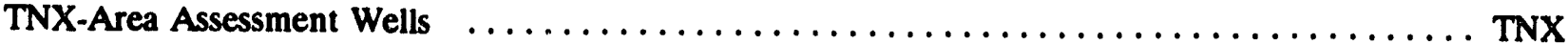

TNX-Area Background Wells Near the TNX Facility at the

Northeast Intersection of SRS Roads 3 and 3-1, approximately

$150 \mathrm{ft}$ off Road 3 (P 26 Cluster)

TNX-Area Pump Test Well for the Congaree

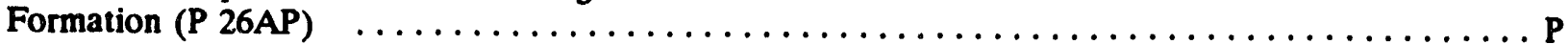

TNX-Area Pump Test Well for the Water Table (P 26DP) $\ldots \ldots \ldots \ldots \ldots \ldots \ldots \ldots \ldots \ldots$

TNX Burying Ground $\ldots \ldots \ldots \ldots \ldots \ldots \ldots \ldots \ldots \ldots \ldots \ldots \ldots \ldots \ldots \ldots \ldots \ldots \ldots \ldots$ 
Unofficial Wells ASP Wells

BG-I Well

H-Area Well

K Well

OBS Wells PW Wells S Well SDS 124 Well Unnamed Well $\mathbf{Z}$ Well

Unnamed Well inside BG wells at the MWMF Unnamed Well

Upper and Lower Tank Farm $\ldots \ldots \ldots \ldots \ldots \ldots \ldots \ldots \ldots \ldots \ldots \ldots \ldots \ldots \ldots$

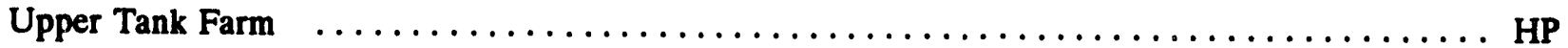

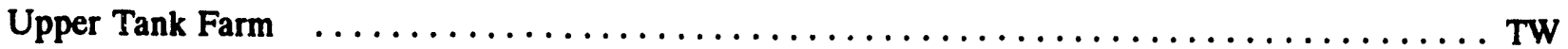

Vapor Probes Adjacent to the Horizontal Wells in M Area $\ldots \ldots \ldots \ldots \ldots \ldots \ldots \ldots$ MHV

Wells between the F-Area Canyon Building and the Naval Fuel Material Facility NBG

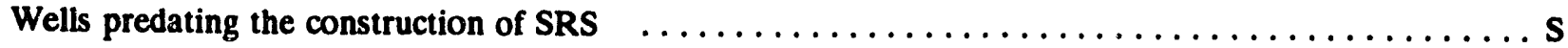

Y-Area Waste Solidification and Disposal Facility $\ldots \ldots \ldots \ldots \ldots \ldots \ldots \ldots \ldots \ldots$ YSC

Z Area ASP Wells OBS Wells PW Wells 
Description

Well Series

Z-Area Saltstone Disposal Site Facility $\quad \ldots \ldots \ldots \ldots \ldots \ldots \ldots \ldots \ldots \ldots \ldots \ldots \ldots \ldots \ldots$

Z-Area Saltstone Facility Background Wells $\ldots \ldots \ldots \ldots \ldots \ldots \ldots \ldots \ldots \ldots \ldots \ldots \ldots$ ZBG

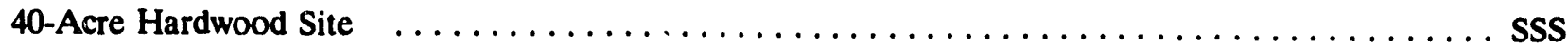




\section{DESCRIPTION INDEX BY AREA}

Description

Well Series

100 AREAS

C Area

C-Area Burning/Rubble Pit $\ldots \ldots \ldots \ldots \ldots \ldots \ldots \ldots \ldots \ldots \ldots \ldots \ldots \ldots \ldots \ldots \ldots \ldots$

C-Area Coal Pile Runoff Containment Basin $\ldots \ldots \ldots \ldots \ldots \ldots \ldots \ldots \ldots \ldots \ldots \ldots$

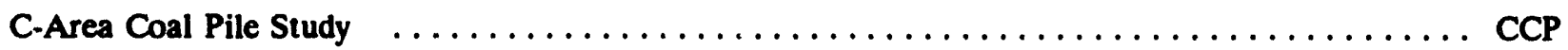

C-Area Disassembly Basin $\ldots \ldots \ldots \ldots \ldots \ldots \ldots \ldots \ldots \ldots \ldots \ldots \ldots \ldots \ldots \ldots \ldots \ldots$

C-Area Reactor Seepage Basins $\ldots \ldots \ldots \ldots \ldots \ldots \ldots \ldots \ldots \ldots \ldots \ldots \ldots \ldots \ldots \ldots \ldots \ldots$

K Area

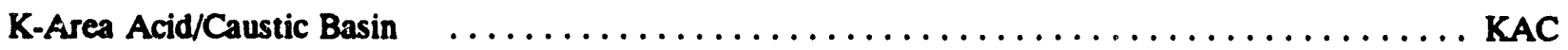

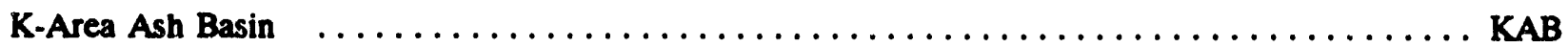

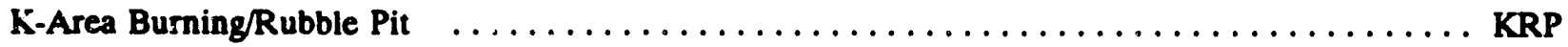

K-Area Coal Pile Runoff Containment Basin $\ldots \ldots \ldots \ldots \ldots \ldots \ldots \ldots \ldots \ldots \ldots$

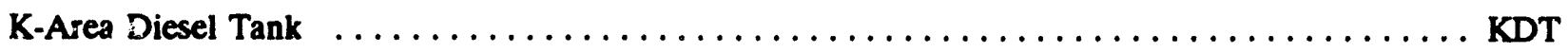

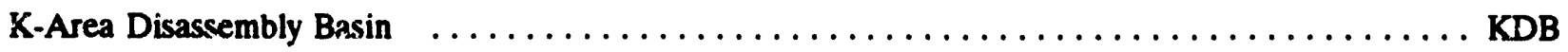

K-Area Reactor Scepage Basin $\quad \ldots \ldots \ldots \ldots \ldots \ldots \ldots \ldots \ldots \ldots \ldots \ldots \ldots \ldots \ldots \ldots \ldots \ldots$

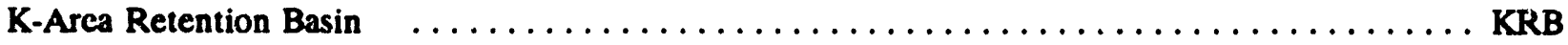

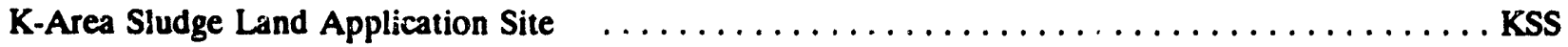

K-Area Water Level Observation Wells $\ldots \ldots \ldots \ldots \ldots \ldots \ldots \ldots \ldots \ldots \ldots \ldots \ldots \ldots \ldots$

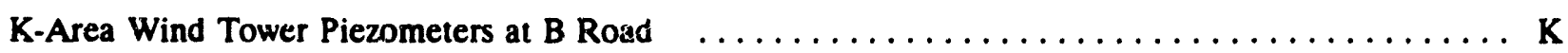

Preliminary Hydrogeologic Evaluation of Potential New

Production Reactor Site Characterization Wells $\ldots \ldots \ldots \ldots \ldots \ldots \ldots \ldots$ NTN, NTS, NTW 
L Area

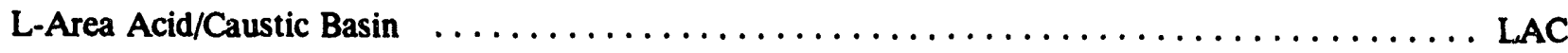

L-Area Burning/Rubble Pit $\ldots \ldots \ldots \ldots \ldots \ldots \ldots \ldots \ldots \ldots \ldots \ldots \ldots \ldots \ldots \ldots$

L-Area Disassembly Basin $\ldots \ldots \ldots \ldots \ldots \ldots \ldots \ldots \ldots \ldots \ldots \ldots \ldots \ldots \ldots$

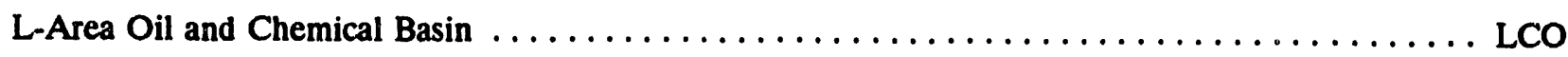

L-Area Reactor Seepage Basin $\quad \ldots \ldots \ldots \ldots \ldots \ldots \ldots \ldots \ldots \ldots \ldots \ldots \ldots \ldots \ldots \ldots$

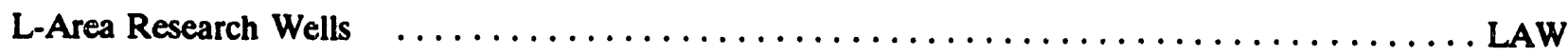

P Area

P-Area Acid/Caustic Basin $\ldots \ldots \ldots \ldots \ldots \ldots \ldots \ldots \ldots \ldots \ldots \ldots \ldots \ldots \ldots \ldots$

P-Area Burning/Rubble Pit $\quad \ldots \ldots \ldots \ldots \ldots \ldots \ldots \ldots \ldots \ldots \ldots \ldots \ldots \ldots \ldots \ldots \ldots \ldots$

P-Area Coal Pile Runoff Containment Basin $\ldots \ldots \ldots \ldots \ldots \ldots \ldots \ldots \ldots \ldots \ldots$

P-Area Disassembly Basin $\quad \ldots \ldots \ldots \ldots \ldots \ldots \ldots \ldots \ldots \ldots \ldots \ldots \ldots \ldots \ldots \ldots \ldots \ldots$

P-Area Reactor Seepage Basins $\ldots \ldots \ldots \ldots \ldots \ldots \ldots \ldots \ldots \ldots \ldots \ldots \ldots \ldots \ldots \ldots \ldots$

R Area

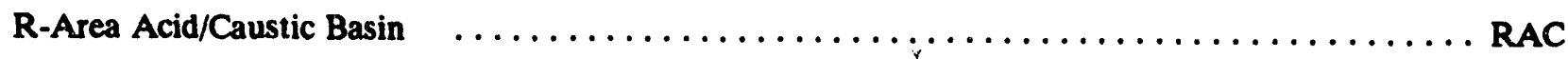

R-Area Burning/Rubble Pits $\quad \ldots \ldots \ldots \ldots \ldots \ldots \ldots \ldots \ldots \ldots \ldots \ldots \ldots \ldots \ldots \ldots \ldots$

R-Area Coal Pile Study $\ldots \ldots \ldots \ldots \ldots \ldots \ldots \ldots \ldots \ldots \ldots \ldots \ldots \ldots \ldots \ldots \ldots$

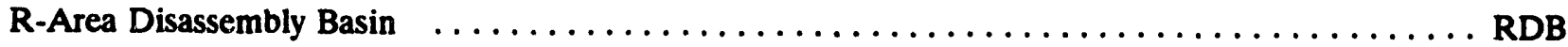

Series A, R-Area Reactor Seepage Basins $\ldots \ldots \ldots \ldots \ldots \ldots \ldots \ldots \ldots \ldots \ldots \ldots \ldots$

Series B, R-Area Reactor Seepage Basins $\quad \ldots \ldots \ldots \ldots \ldots \ldots \ldots \ldots \ldots \ldots \ldots \ldots \ldots$

Series C, R-Area Reactor Seepage Basins $\quad \ldots \ldots \ldots \ldots \ldots \ldots \ldots \ldots \ldots \ldots \ldots \ldots \ldots$ RSC

Series D, between R-Area Reactor Seepage Basins

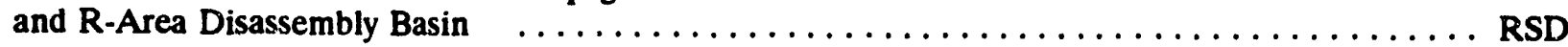

Series DW, R-Area Reactor Seepage Basins Dry Monitoring Wells 
Series E, R-Area Reactor Seepage Basins RSE

Series F, R-Area Reactor Seepage Basins RSF

200 AREAS

Burial Grounds (E Area) ${ }^{1}$

Bentonite Test Pit Wells, across from the entrance to the Burial Grounds on Road $\mathrm{E} \ldots \ldots \ldots \ldots \ldots \ldots \ldots \ldots \ldots \ldots \ldots \ldots \ldots \ldots \ldots \ldots$

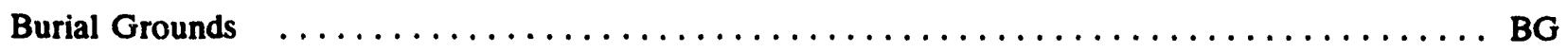

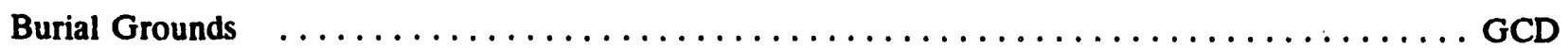

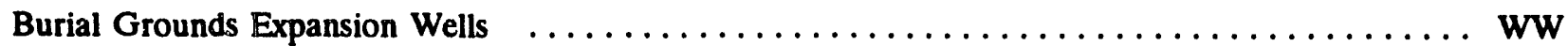

Burial Grounds Grid Wells $\ldots \ldots \ldots \ldots \ldots \ldots \ldots \ldots \ldots \ldots \ldots \ldots \ldots \ldots \ldots \ldots$

Burial Grounds Perimeter Wells $\quad \ldots \ldots \ldots \ldots \ldots \ldots \ldots \ldots \ldots \ldots \ldots \ldots \ldots$

E Area Vaults near the Burial Grounds $\ldots \ldots \ldots \ldots \ldots \ldots \ldots \ldots \ldots \ldots \ldots \ldots \ldots$

Just north of the Burial Grounds $\ldots \ldots \ldots \ldots \ldots \ldots \ldots \ldots \ldots \ldots \ldots \ldots \ldots$

Just outside the fence surrounding the Burial Grounds;

BG 43, 52, and 67 are inside the fence $\ldots \ldots \ldots \ldots \ldots \ldots \ldots \ldots \ldots \ldots \ldots \ldots \ldots \ldots$

Mixed Waste Management Facility (MWMF) Borrow Pit $\ldots \ldots \ldots \ldots \ldots \ldots \ldots \ldots$ Kell

Series A, Monitoring Grid Wells for Burial Grounds $\ldots \ldots \ldots \ldots \ldots \ldots \ldots \ldots \ldots$

Series C, Monitoring Grid Wells for Burial Grounds $\ldots \ldots \ldots \ldots \ldots \ldots \ldots \ldots \ldots \ldots$

Series E, Monitoring Grid Wells for Burial Grounds $\ldots \ldots \ldots \ldots \ldots \ldots \ldots \ldots \ldots$ MGE

Series $G$, Monitoring Grid Wells for Burial Grounds $\ldots \ldots \ldots \ldots \ldots \ldots \ldots \ldots \ldots$

Series I, Monitoring Grid Wells for Burial Grounds $\ldots \ldots \ldots \ldots \ldots \ldots \ldots \ldots \ldots \ldots$

Special Burial Wells in the Burial Grounds $\ldots \ldots \ldots \ldots \ldots \ldots \ldots \ldots \ldots \ldots \ldots \ldots \ldots$

'The Burial Grounds wells are in the 600 General Area; however, these wells are closely connected to activities in the 200 Areas. 
Description

Well Series

Unnamed Well inside BG wells at the MWMF Unnamed Well

Burma Road Rubble Pit BRR

F Area

F Area BG-I Well

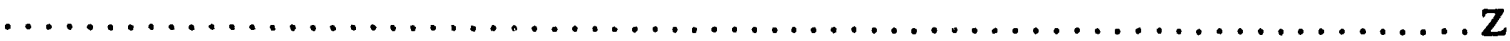

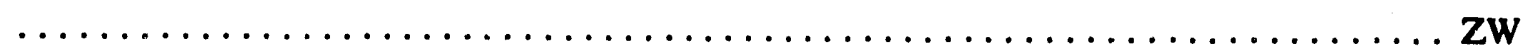

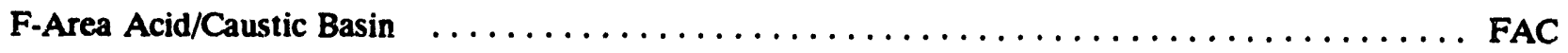

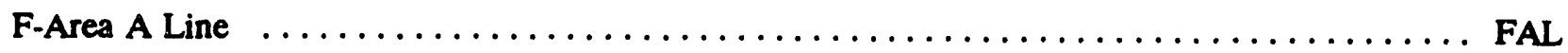

F-Area Burning/Rubble Pits $\ldots \ldots \ldots \ldots \ldots \ldots \ldots \ldots \ldots \ldots \ldots \ldots \ldots \ldots \ldots$

F-Area Canyon Building $\quad \ldots \ldots \ldots \ldots \ldots \ldots \ldots \ldots \ldots \ldots \ldots \ldots \ldots \ldots \ldots \ldots \ldots$

F-Area Coal Pile Runoff Containment Basin $\ldots \ldots \ldots \ldots \ldots \ldots \ldots \ldots \ldots \ldots \ldots$

F-Area Effluent Treatment Cooling Water Basin $\ldots \ldots \ldots \ldots \ldots \ldots \ldots \ldots \ldots \ldots \ldots$ FET

F-Area Hydrology Cluster $\quad \ldots \ldots \ldots \ldots \ldots \ldots \ldots \ldots \ldots \ldots \ldots \ldots \ldots \ldots \ldots \ldots$ FC

F-Area Microbiology Wells $\ldots \ldots \ldots \ldots \ldots \ldots \ldots \ldots \ldots \ldots \ldots \ldots \ldots \ldots \ldots \ldots$

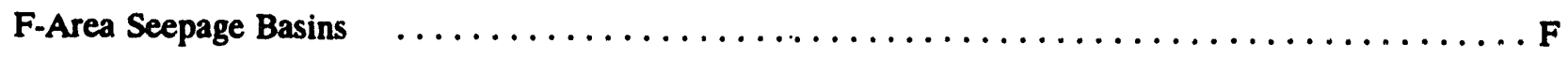
FSB

F-Area Seepage Basins Production Well FSB 1TA Well

F-Area Seepage Basins Vertical Radionuclide

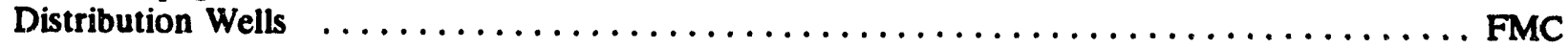

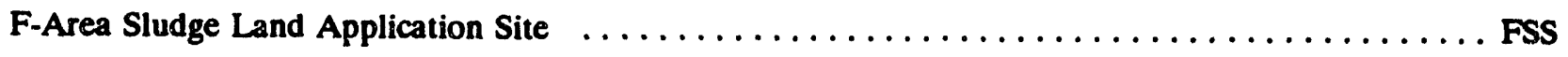

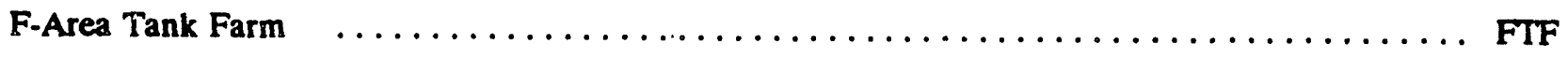

$241-\mathrm{F}$

Old F-Area Seepage Basin $\ldots \ldots \ldots \ldots \ldots \ldots \ldots \ldots \ldots \ldots \ldots \ldots \ldots \ldots \ldots$

Wells between the F-Area Canyon Building and the Naval Fuel Material Facility NBG 
Description

Well Series

H Area

Aquifer Characterization Test Wells in $\mathrm{H}$ Area between the Burial Grounds and Road $4 \ldots \ldots \ldots \ldots \ldots \ldots \ldots \ldots \ldots \ldots \ldots \ldots \ldots \ldots$

H Area H-Area Well

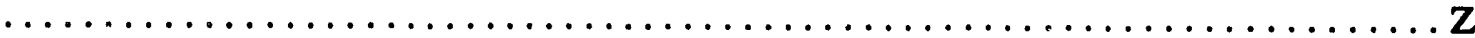

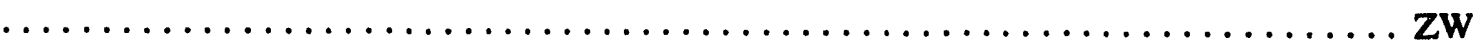

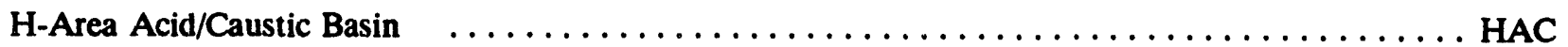

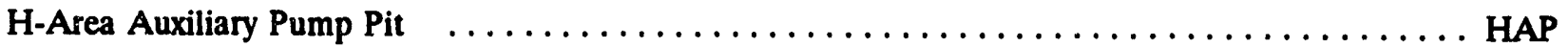

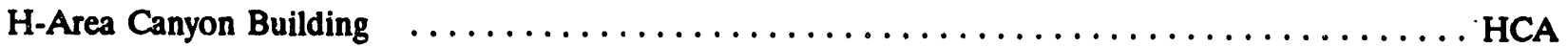

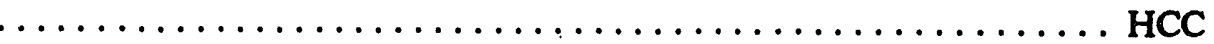

H-Area Coal Pile Runoff Containment Basin $\ldots \ldots \ldots \ldots \ldots \ldots \ldots \ldots \ldots \ldots$

H-Area Effluent Treatment Cooling Water Basin $\ldots \ldots \ldots \ldots \ldots \ldots \ldots \ldots \ldots \ldots \ldots$

H-Area Hydrology Cluster $\ldots \ldots \ldots \ldots \ldots \ldots \ldots \ldots \ldots \ldots \ldots \ldots \ldots \ldots \ldots \ldots \ldots \ldots$

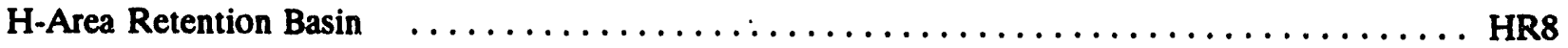

H-Area Seepage Basins $\quad \ldots \ldots \ldots \ldots \ldots \ldots \ldots \ldots \ldots \ldots \ldots \ldots \ldots \ldots \ldots \ldots \ldots$ HSB

H-Area Seepage Basins Production Well $\ldots \ldots \ldots \ldots \ldots \ldots \ldots \ldots \ldots \ldots$ HSB 1TB Well

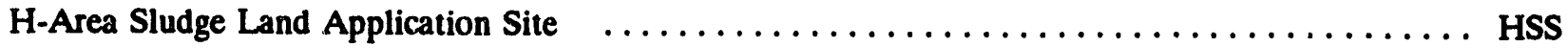

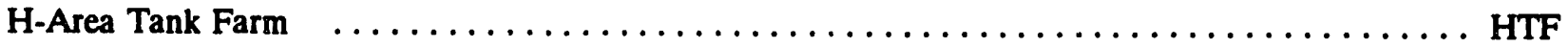

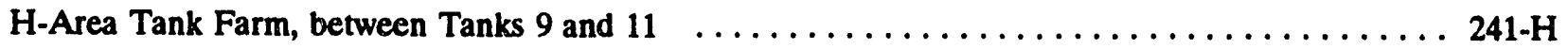

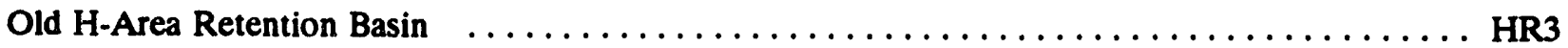

Swamp area southeast of $\mathrm{H}$-Area Seepage Basins $\ldots \ldots \ldots \ldots \ldots \ldots \ldots \ldots \ldots \ldots \ldots \ldots$

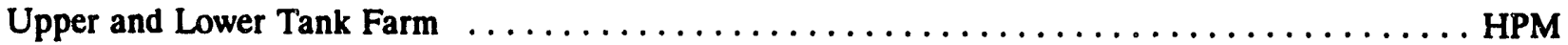

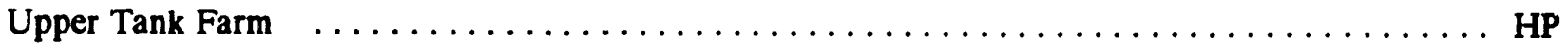


Description

Well Series

S Area

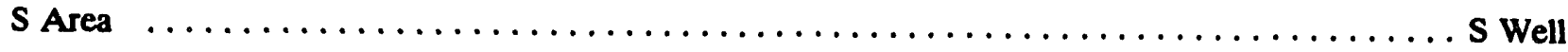

S-Area Defense Waste Processing Facility (DWPF) $\ldots \ldots \ldots \ldots \ldots \ldots \ldots \ldots \ldots \ldots \ldots$

S-Area Defense Waste Processing Facility (DWPF)

Background Wells

SBG

S-Area Defense Waste Processing Facility (DWPF)

Boreholes and Piezometers

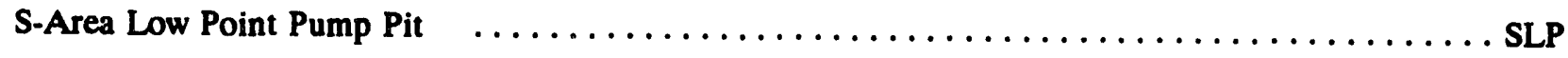

S-Area Vitrification Building $\ldots \ldots \ldots \ldots \ldots \ldots \ldots \ldots \ldots \ldots \ldots \ldots \ldots \ldots \ldots \ldots$

Y Area

Y-Area Waste Solidification and Disposal Facility $\ldots \ldots \ldots \ldots \ldots \ldots \ldots \ldots \ldots \ldots$

Z Area

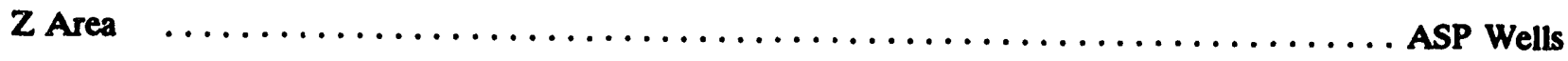

OBS Wells

PW Wells

Z Well

Z-Area Low Point Drain Tank $\quad \ldots \ldots \ldots \ldots \ldots \ldots \ldots \ldots \ldots \ldots \ldots \ldots \ldots \ldots \ldots \ldots \ldots \ldots$

Z-Area Piezometer Wells $\ldots \ldots \ldots \ldots \ldots \ldots \ldots \ldots \ldots \ldots \ldots \ldots \ldots \ldots \ldots \ldots$

Z-Area Saltstone Disposal Site Facility $\quad \ldots \ldots \ldots \ldots \ldots \ldots \ldots \ldots \ldots \ldots \ldots \ldots$ sDS

Z-Area Saltstone Facility Background Wells $\ldots \ldots \ldots \ldots \ldots \ldots \ldots \ldots \ldots \ldots \ldots \ldots \ldots$

300/700 AREAS

A-Area Background Well near Firing Range $\ldots \ldots \ldots \ldots \ldots \ldots \ldots \ldots \ldots \ldots \ldots \ldots \ldots \ldots$

A-Area Burning/Rubble Pits $\quad \ldots \ldots \ldots \ldots \ldots \ldots \ldots \ldots \ldots \ldots \ldots \ldots \ldots \ldots \ldots \ldots \ldots$

A-Area Cluster Perimeter Wells $\quad \ldots \ldots \ldots \ldots \ldots \ldots \ldots \ldots \ldots \ldots \ldots \ldots \ldots \ldots \ldots \ldots$

A-Area Coal Pile Runoff Containment Basin $\ldots \ldots \ldots \ldots \ldots \ldots \ldots \ldots \ldots \ldots \ldots \ldots$ 
Description

Well Series

A-Airea Metals Burning Pit $\ldots \ldots \ldots \ldots \ldots \ldots \ldots \ldots \ldots \ldots \ldots \ldots \ldots \ldots \ldots \ldots \ldots \ldots \ldots$

Geophysical Test Holes for the Horizontal

Well Characterization Study in M-Area MHM

M-Area Hazardous Waste Management Facility (HWMF) $\ldots \ldots \ldots \ldots \ldots \ldots \ldots \ldots$ MSB

M-Area Plume Definition Wells $\ldots \ldots \ldots \ldots \ldots \ldots \ldots \ldots \ldots \ldots \ldots \ldots \ldots \ldots \ldots \ldots$

M-Area Recovery ${ }^{W e l l s} \quad \ldots \ldots \ldots \ldots \ldots \ldots \ldots \ldots \ldots \ldots \ldots \ldots \ldots \ldots \ldots \ldots \ldots \ldots$

M-Area Settling Basin (also used for plume definition) $\quad \ldots \ldots \ldots \ldots \ldots \ldots \ldots \ldots \ldots$ MSB

M-Area Vadose Zone Extraction Wells $\ldots \ldots \ldots \ldots \ldots \ldots \ldots \ldots \ldots \ldots \ldots \ldots \ldots \ldots \ldots$

M-Area Vadose Zone Vapor Probes $\ldots \ldots \ldots \ldots \ldots \ldots \ldots \ldots \ldots \ldots \ldots \ldots \ldots \ldots \ldots$

M-Area Wells Required by the SRS Hazardous Waste Permit $\ldots \ldots \ldots \ldots \ldots \ldots \ldots \ldots$ MSB

Metallurgical Laboratory Seepage Basin $\ldots \ldots \ldots \ldots \ldots \ldots \ldots \ldots \ldots \ldots \ldots \ldots \ldots$ AMB

Miscellaneous Chemical Basin $\ldots \ldots \ldots \ldots \ldots \ldots \ldots \ldots \ldots \ldots \ldots \ldots \ldots \ldots \ldots \ldots$

Motor Shop Oil Basin $\ldots \ldots \ldots \ldots \ldots \ldots \ldots \ldots \ldots \ldots \ldots \ldots \ldots \ldots \ldots \ldots \ldots$ AOB

Savannah River Laboratory (SRL) Monitoring Wells for the

Horizontal Well Characterization Study in $\mathbf{M}$ Area $\ldots \ldots \ldots \ldots \ldots \ldots \ldots \ldots \ldots \ldots$ MHT

Savannah River Laboratory (SRL) Seepage Basins $\ldots \ldots \ldots \ldots \ldots \ldots \ldots \ldots \ldots \ldots \ldots$ ASB

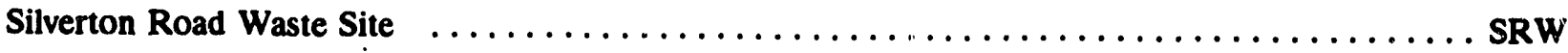

Vapor Probes Adjacent to the Horizontal Wells in M Area $\ldots \ldots \ldots \ldots \ldots \ldots \ldots \ldots$ MHV

400 AREA

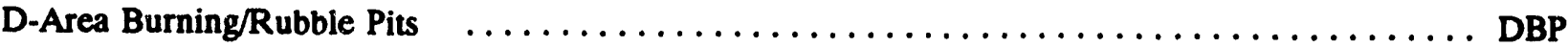

D-Area Coal Pile Runoff Containment Basin

and Ash Basins $\ldots \ldots \ldots \ldots \ldots \ldots \ldots \ldots \ldots \ldots \ldots \ldots \ldots \ldots \ldots \ldots \ldots \ldots \ldots \ldots$

D-Area Oil Disposal Basin $\quad \ldots \ldots \ldots \ldots \ldots \ldots \ldots \ldots \ldots \ldots \ldots \ldots \ldots \ldots \ldots$ DOB

\section{AREAS}

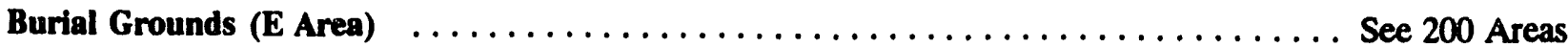


Description

Well Series

Centrul Shops

Central Shops Burning/Rubble Pit south

of the Ford Building Seepage Basin

CBR

Centra! Shops Burning/Rubble Pits CSR

Central Shops Diesel Spill Characterization and Remediation Wells CSD

Fire Department Training Facility Cso

Ford Building Seepage Basin HXB

Hazardous Waste Storage Facility HWS

Preliminary Hydrogeologic Evaluation of Potential New

Production Reactor Site Characterization Wells NPM

\section{General}

Background Well near Hawthorne Fire Tower $\ldots \ldots \ldots \ldots \ldots \ldots \ldots \ldots \ldots \ldots \ldots$ GBW

B-Area Microbiology Wells $\ldots \ldots \ldots \ldots \ldots \ldots \ldots \ldots \ldots \ldots \ldots \ldots \ldots \ldots \ldots \ldots \ldots$

Characterization piezometers for the proposed

Sanitary Landfill, Site B, off E2 Road $\ldots \ldots \ldots \ldots \ldots \ldots \ldots \ldots \ldots \ldots \ldots \ldots \ldots$ BTP

Chemicals, Metals, and Pesticides (CMP) Pits $\ldots \ldots \ldots \ldots \ldots \ldots \ldots \ldots \ldots \ldots \ldots \ldots$

Hazardous Waste/Mixed Waste Disposal Facility $\ldots \ldots \ldots \ldots \ldots \ldots \ldots \ldots \ldots \ldots$ HMD

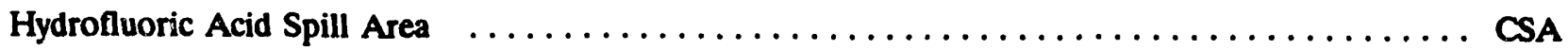

Interim Waste Technology Sitc Characterization Wells,

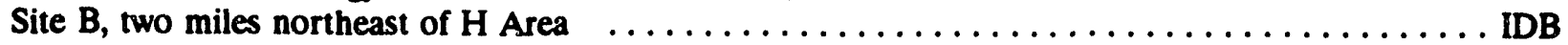

Interim Waste Technology Site Characterization Wells,

Site $P$, adjacent to Site $Q$, and located south of the

TC-1 Area and north of Highway 125

Interim Waste Technology Site Characterization Wells,

Site $Q$, adjacent to Site $P$, and located south of the

TC-1 Area and north of Highway 125

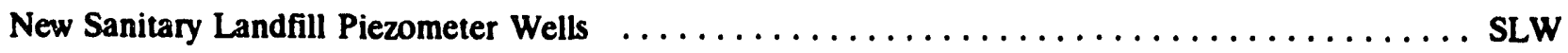

Par Pond Sludge Land Application Site $\ldots \ldots \ldots \ldots \ldots \ldots \ldots \ldots \ldots \ldots \ldots \ldots \ldots$ PSS 
Potential Hazardous Waste/Mixed Waste Disposal Facility $\ldots \ldots \ldots \ldots \ldots \ldots \ldots \ldots \ldots \ldots$ MWD

Preliminary Hydrogeologic Evaluation of Potential New

Production Reactor Site Characterization Wells $\ldots \ldots \ldots \ldots \ldots \ldots \ldots \ldots \ldots \ldots$ NPN

Road A Chemical Basin (Baxley Road) $\ldots \ldots \ldots \ldots \ldots \ldots \ldots \ldots \ldots \ldots \ldots \ldots \ldots \ldots$

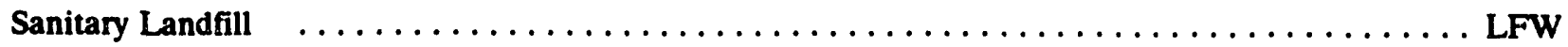

Sewage Sludge Application Sites $\ldots \ldots \ldots \ldots \ldots \ldots \ldots \ldots \ldots \ldots \ldots \ldots \ldots \ldots \ldots \ldots$

TNX

New TNX Seepage Basin $\ldots \ldots \ldots \ldots \ldots \ldots \ldots \ldots \ldots \ldots \ldots \ldots \ldots \ldots \ldots \ldots \ldots \ldots$

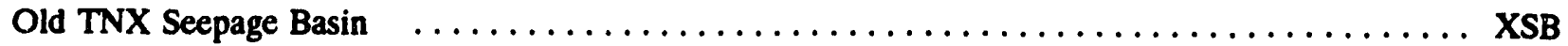

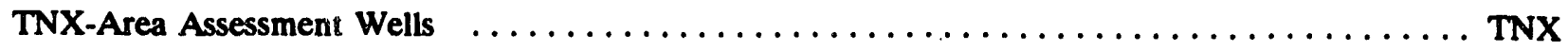

TNX-Area Background Wells Near the TNX Facility at the Northeast Intersection of SRS Roads 3 and 3-1, approx-

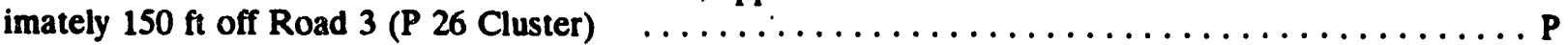

TNX-Area Pump Test Well for the Congaree

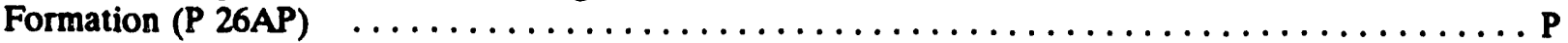

TNX-Area Pump Test Well for the Water Table (P 26DP) $\ldots \ldots \ldots \ldots \ldots \ldots \ldots \ldots \ldots \ldots$

TNX Burying Ground $\quad \ldots \ldots \ldots \ldots \ldots \ldots \ldots \ldots \ldots \ldots \ldots \ldots \ldots \ldots \ldots \ldots \ldots$ 
This page was left blank intentionally. 


\section{APPENDIX A}

\section{AREA MAPS}

The following maps show the location of the EPD/EMS wells. These maps were prepared in AutoCAD by Exploration Resources of Athens, Georgia. Disk and hard copies are available from EPD/EMS. Wells in a series may appear on more than one map. Figure 1 coordinates are based on latitude and longitude. All other figure coordinates are based on SRS coordinates. 


\section{Area Maps}

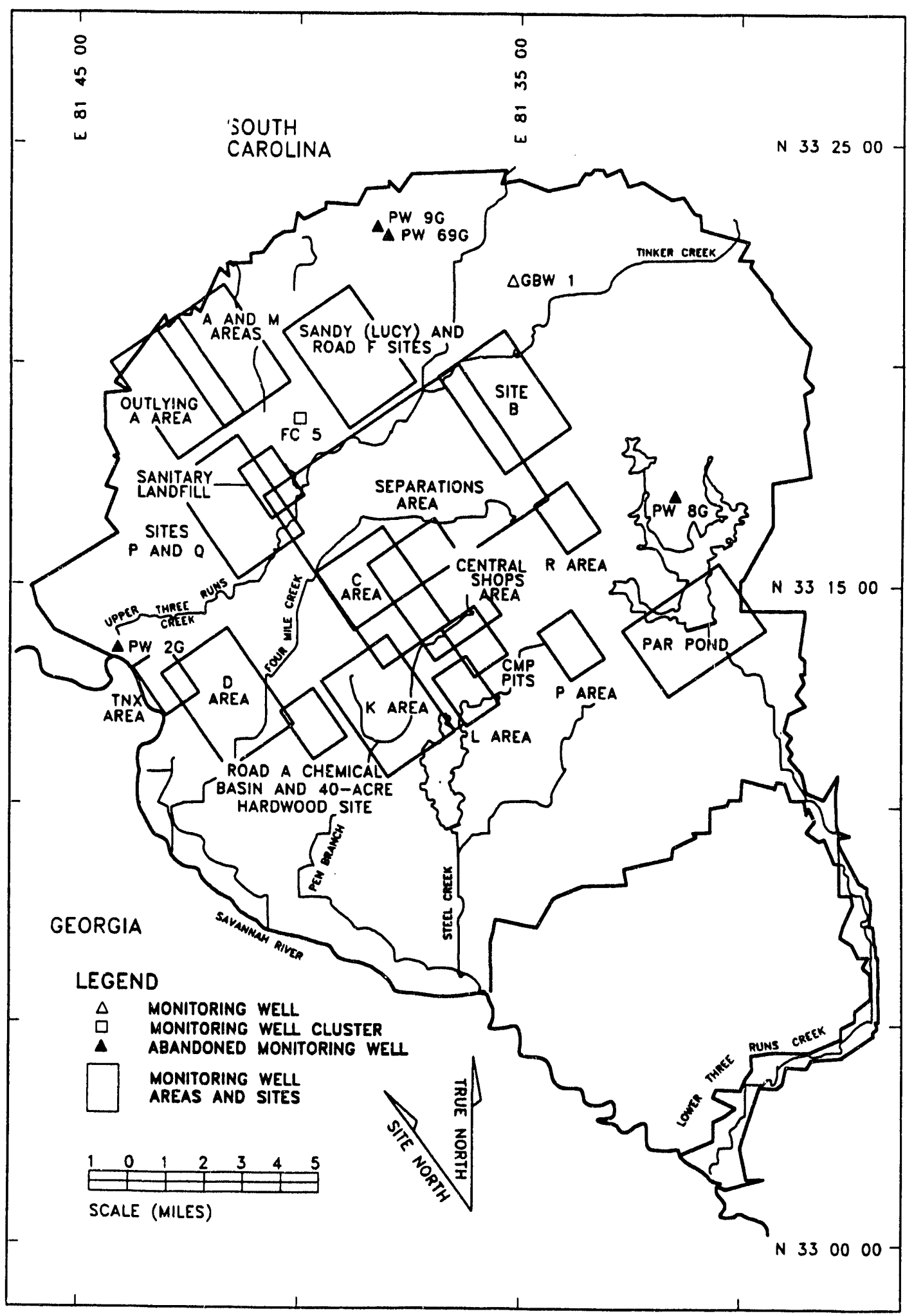

Figure A-1. Areas, Sites, the GBW Well, the FC 5 cluster, and PW Production Wells at the Savannah River Site 
Area Maps

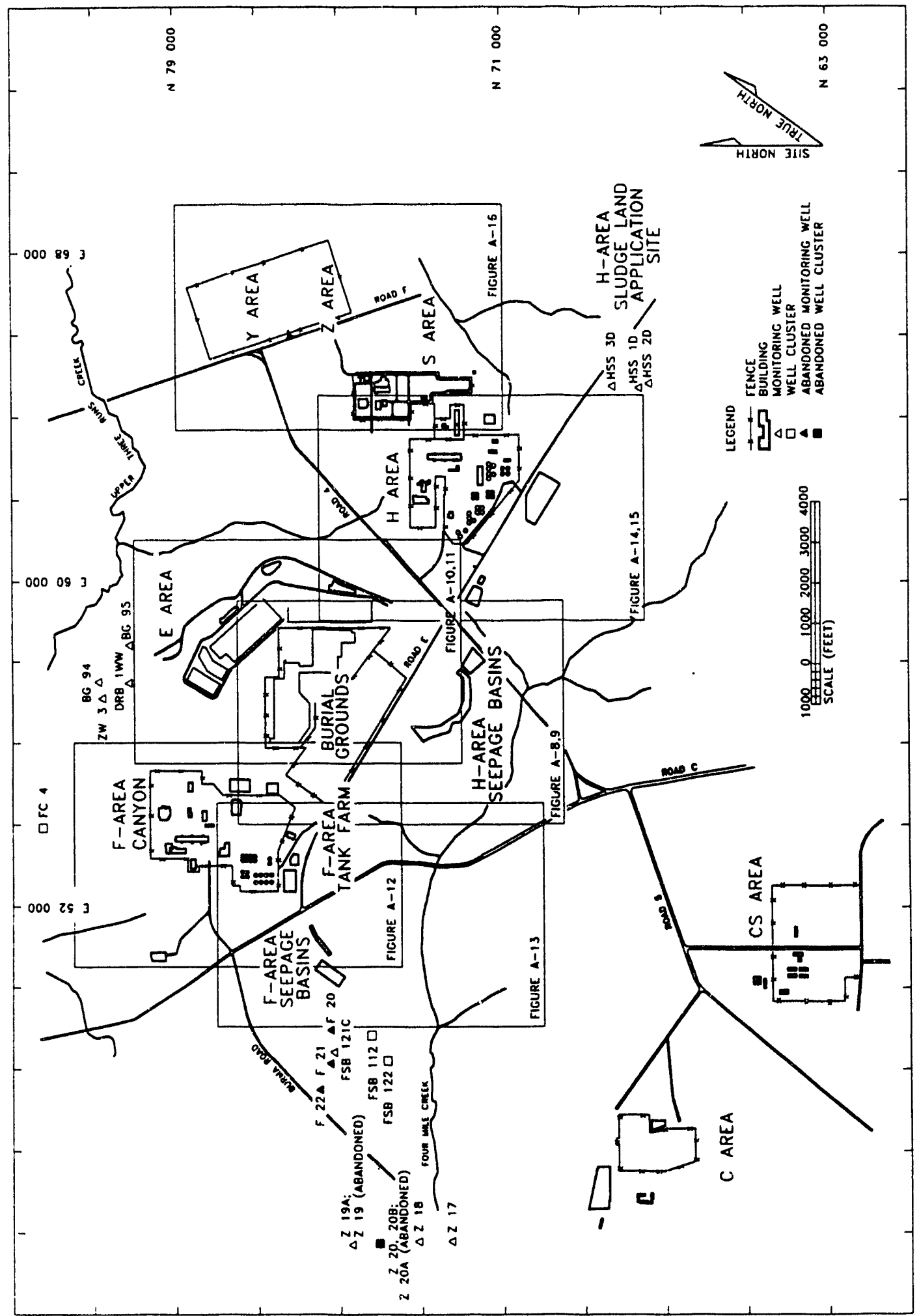

Figure A-2. C Area, Separations Area, and Nearby Wells 


\section{Area Maps}

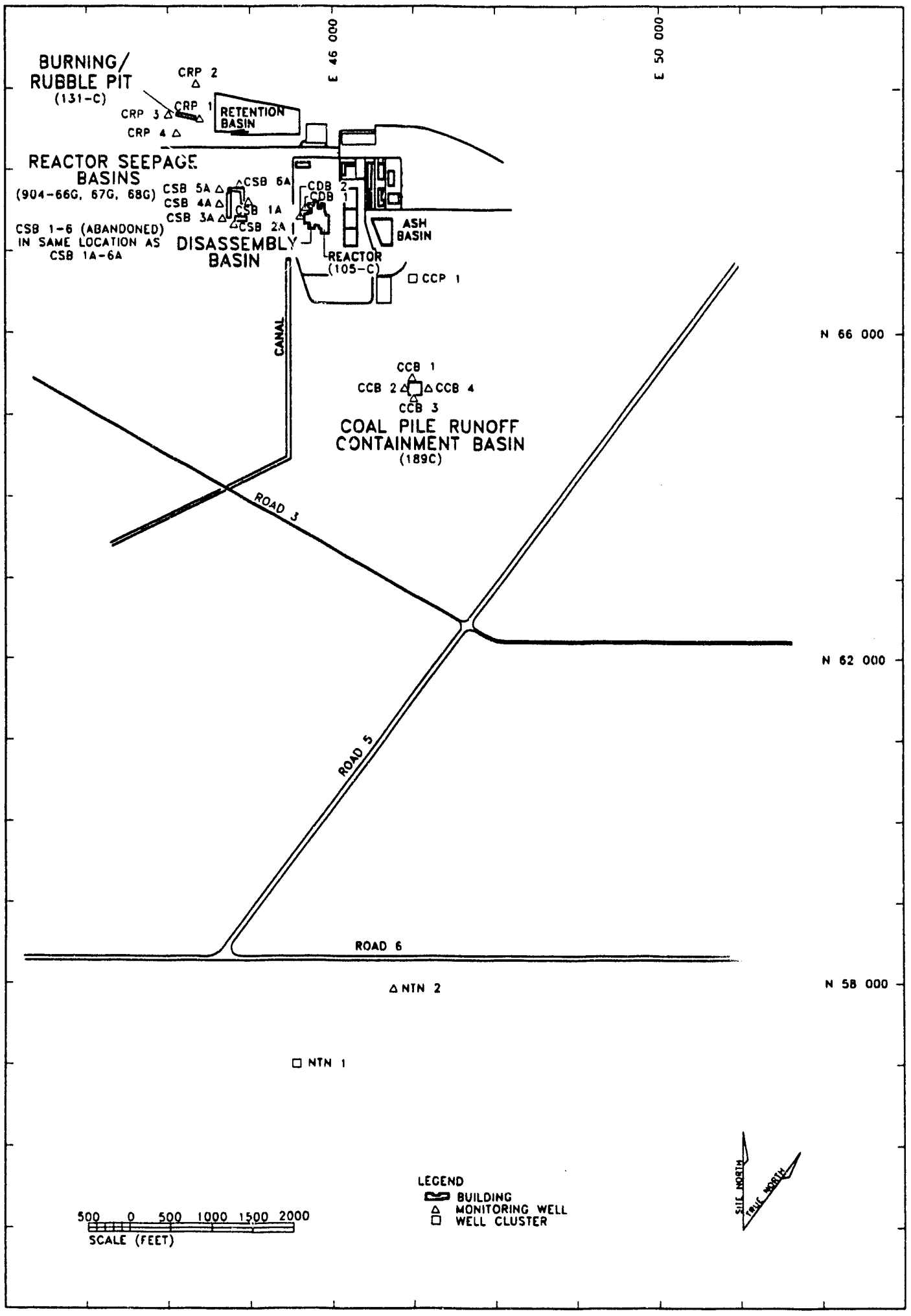

Figure A-3. Groundwater Monitoring Wells In and Near C Area 


\section{Area Maps}

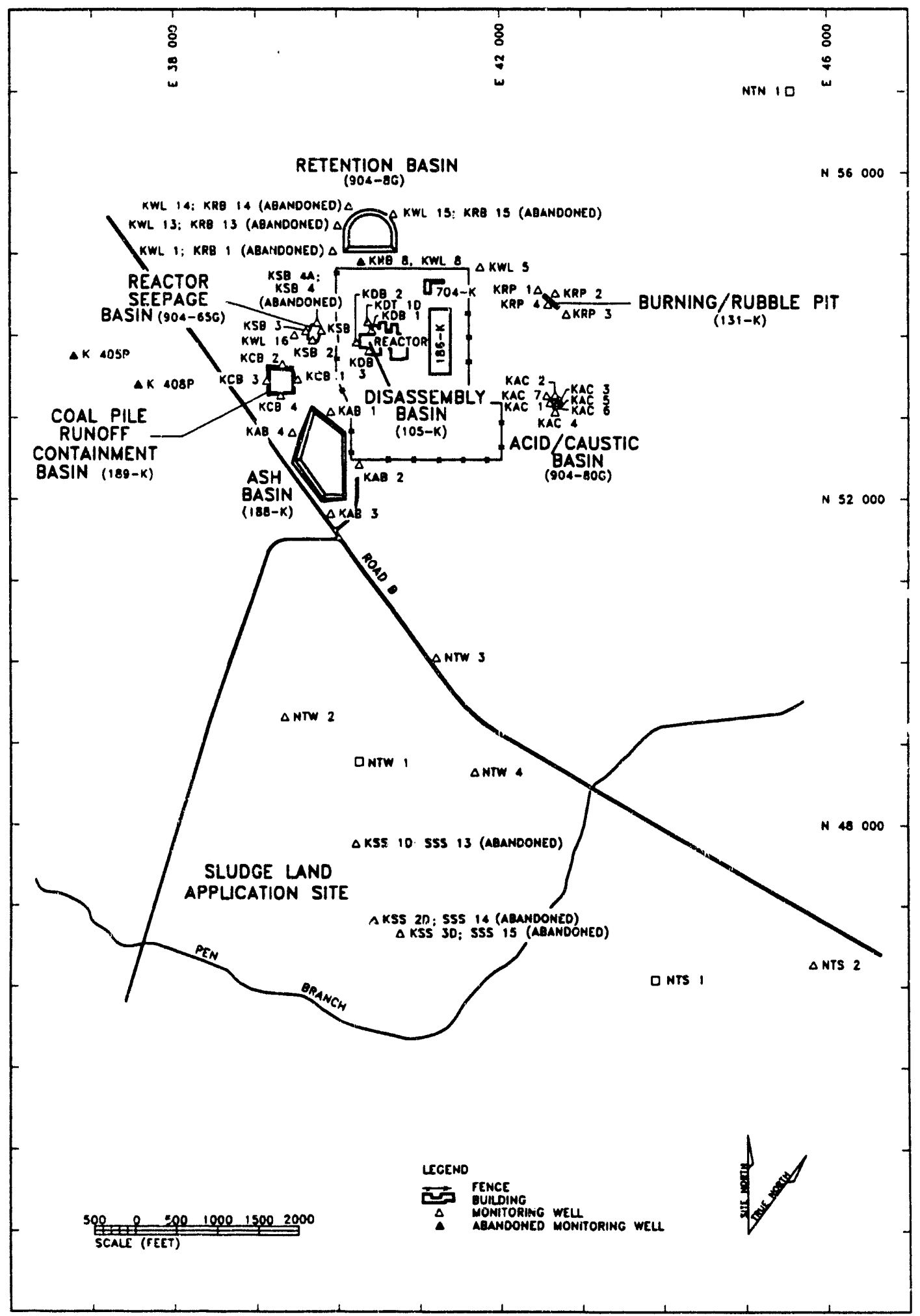

Figure A-4. Groundwater Monitoring Wells In and Near K Area 
Area Maps

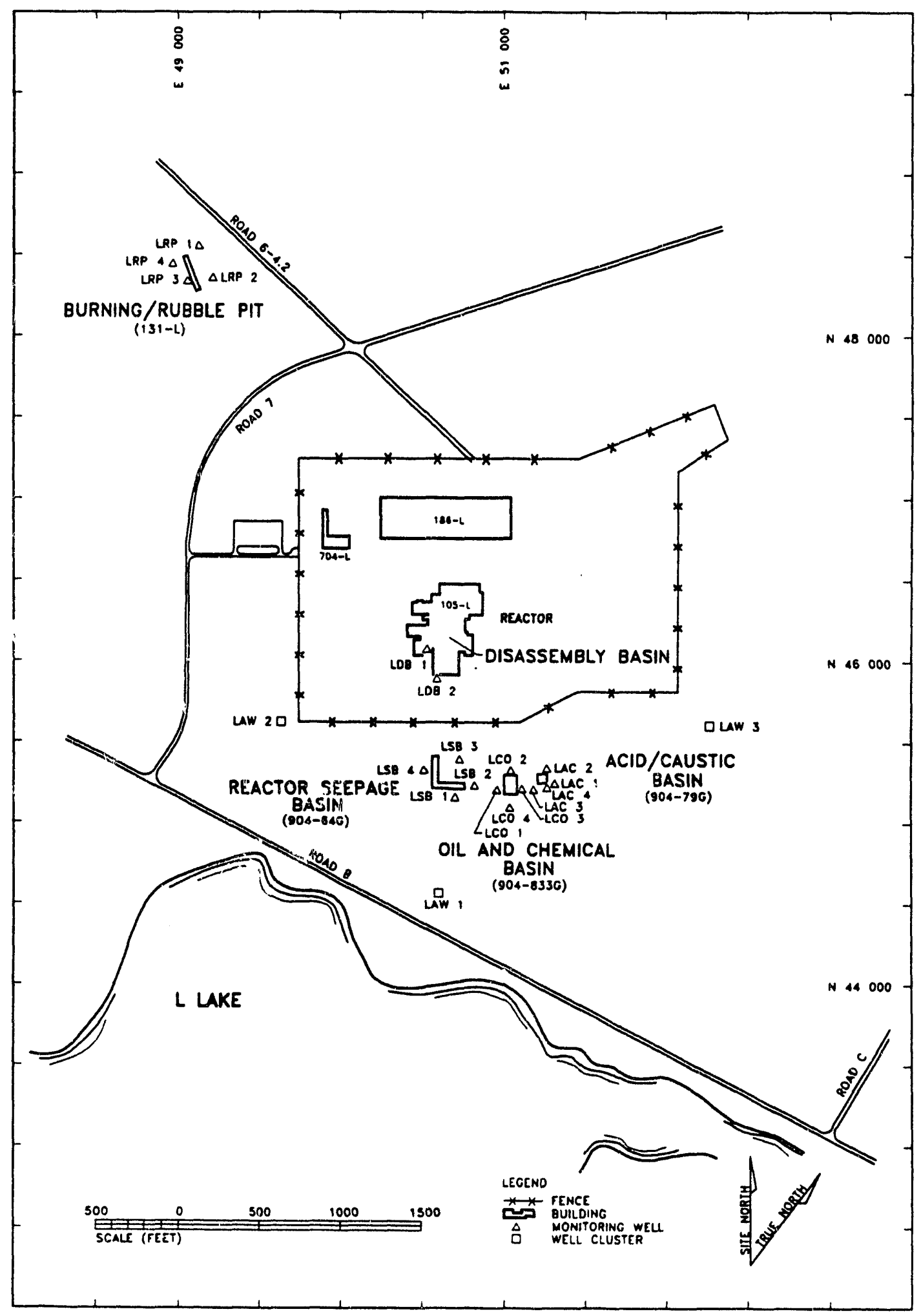

Figure A-5. Groundwater Monitoring Wells at L Area 


\section{Area Maps}

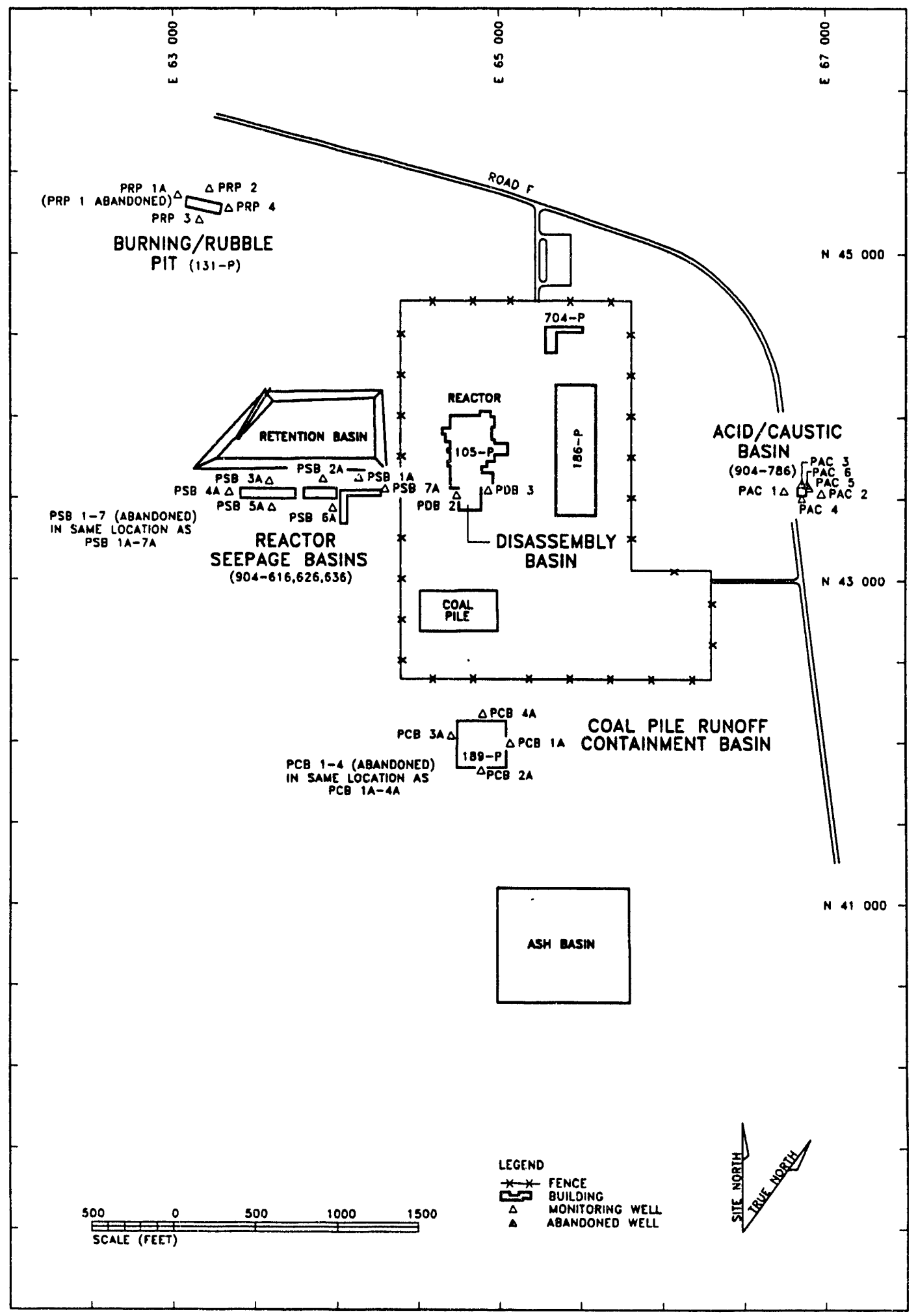

Figure A-6. Groundwater Monitoring Welis at P Area 


\section{Area Maps}

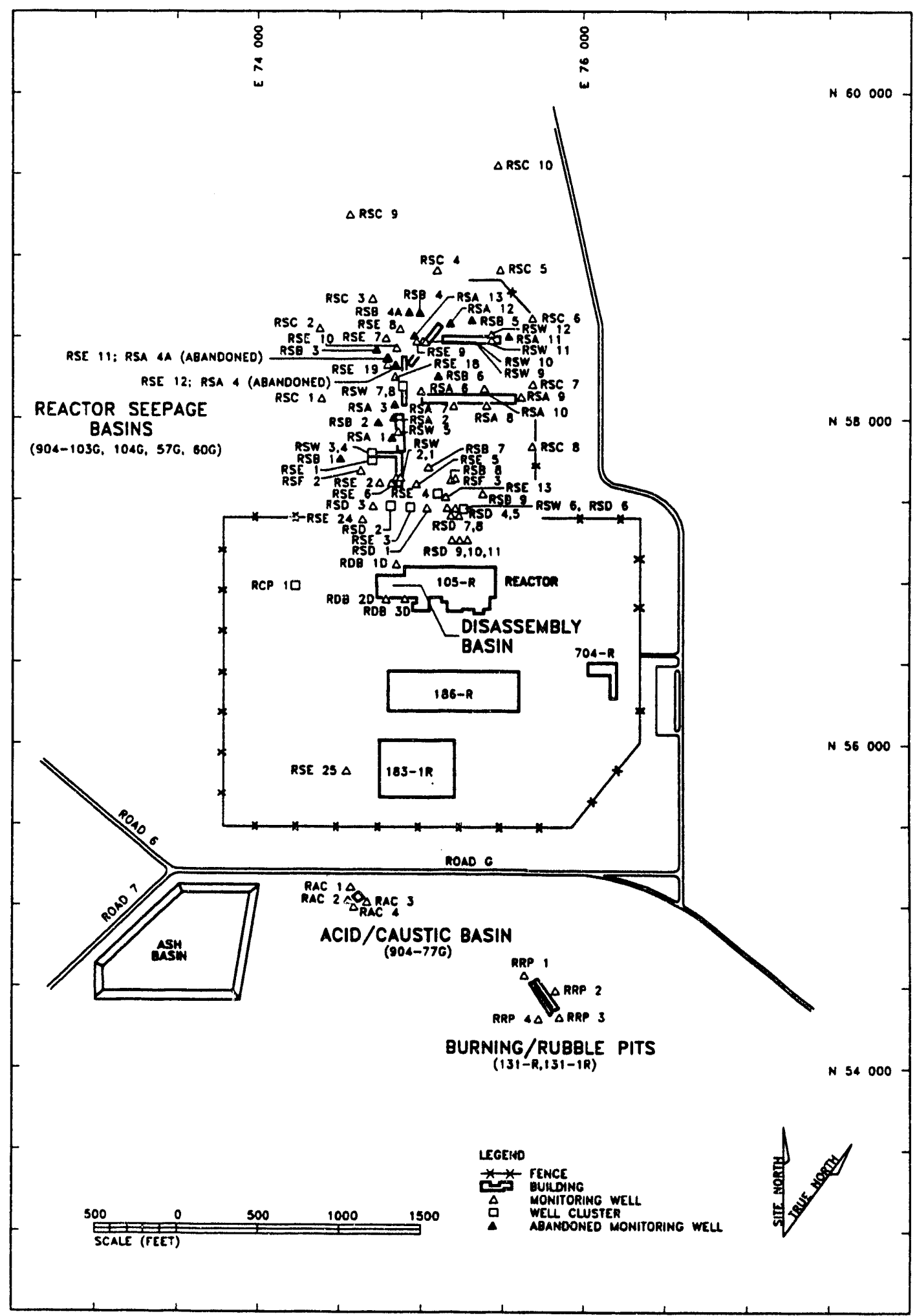

Figure A-7. Groundwater Monitoring Wells at R Area 


\section{Area Maps}

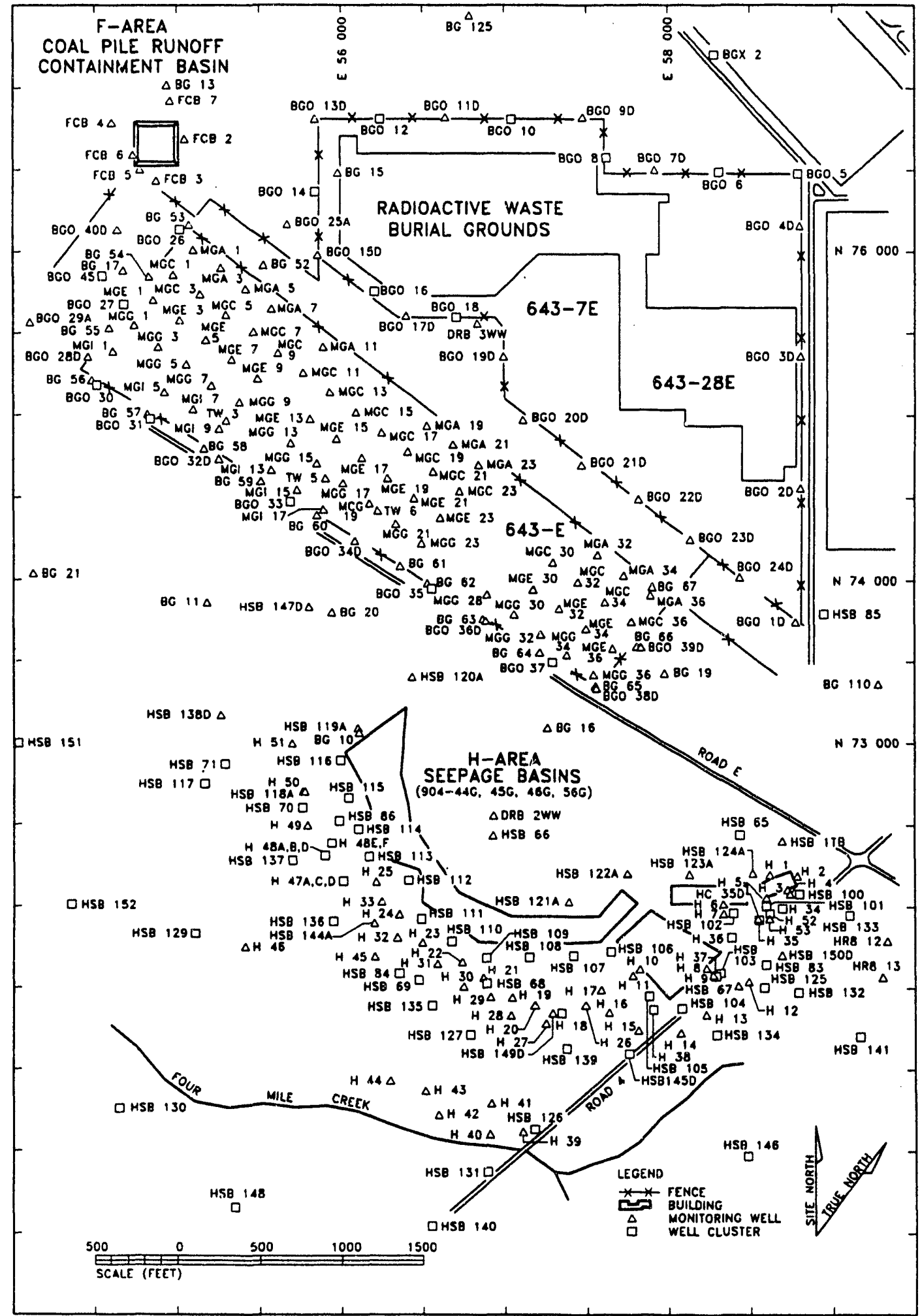

Figure A-8. Active Groundwater Monitoring Wells In and Near E Area and H-Area Seepage Basins 


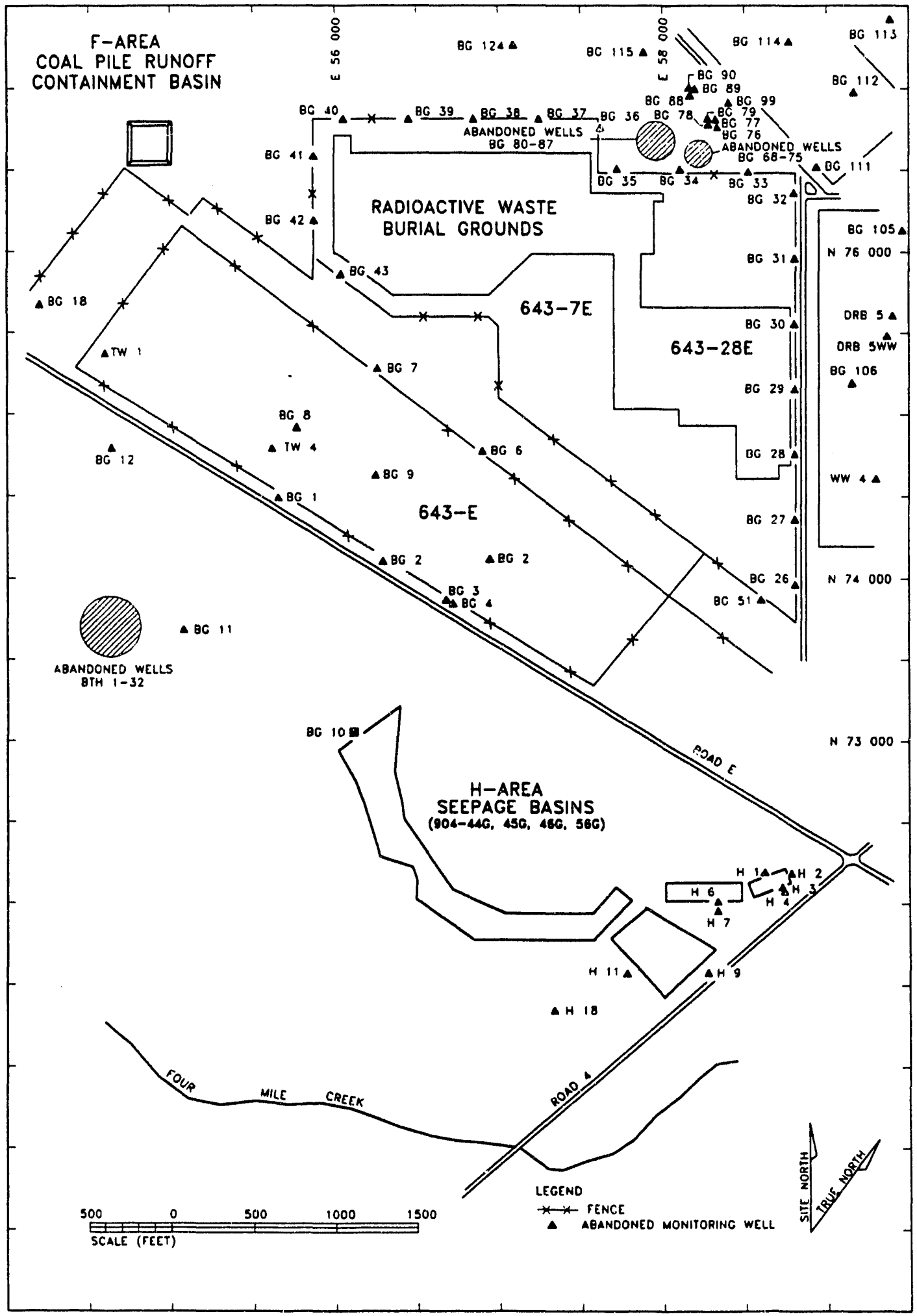

Figure A-9. Abandoned Groundwater Monitoring Wells In and Near E Area and H-Area Seepage Basins 


\section{Area Maps}

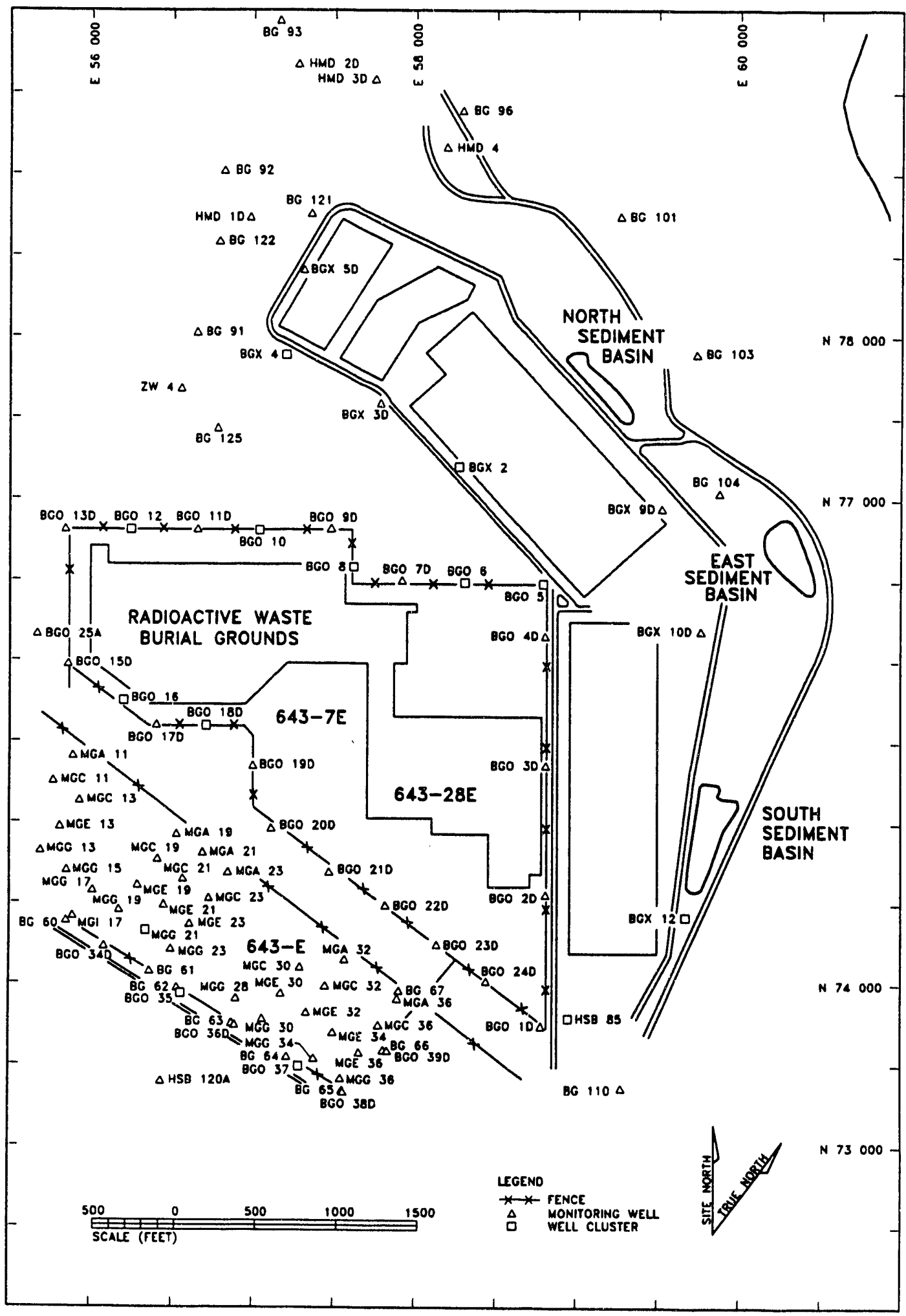

Figure A-10. Active Groundwater Monitoring Wells at the North Part of E Area 


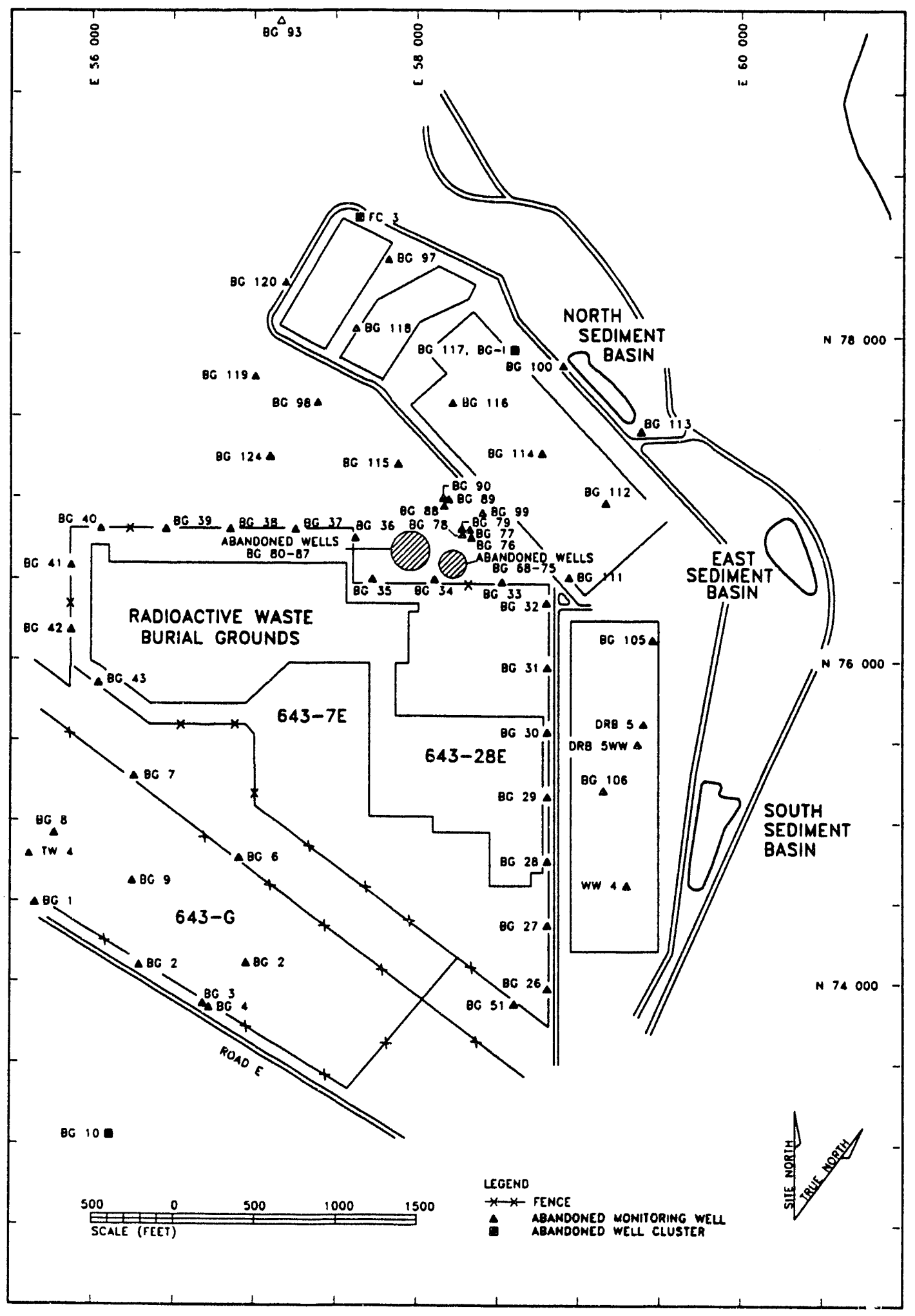

Figure A-11. Abandoned Groundwater Monitoring Wells at the North Part of E Area 


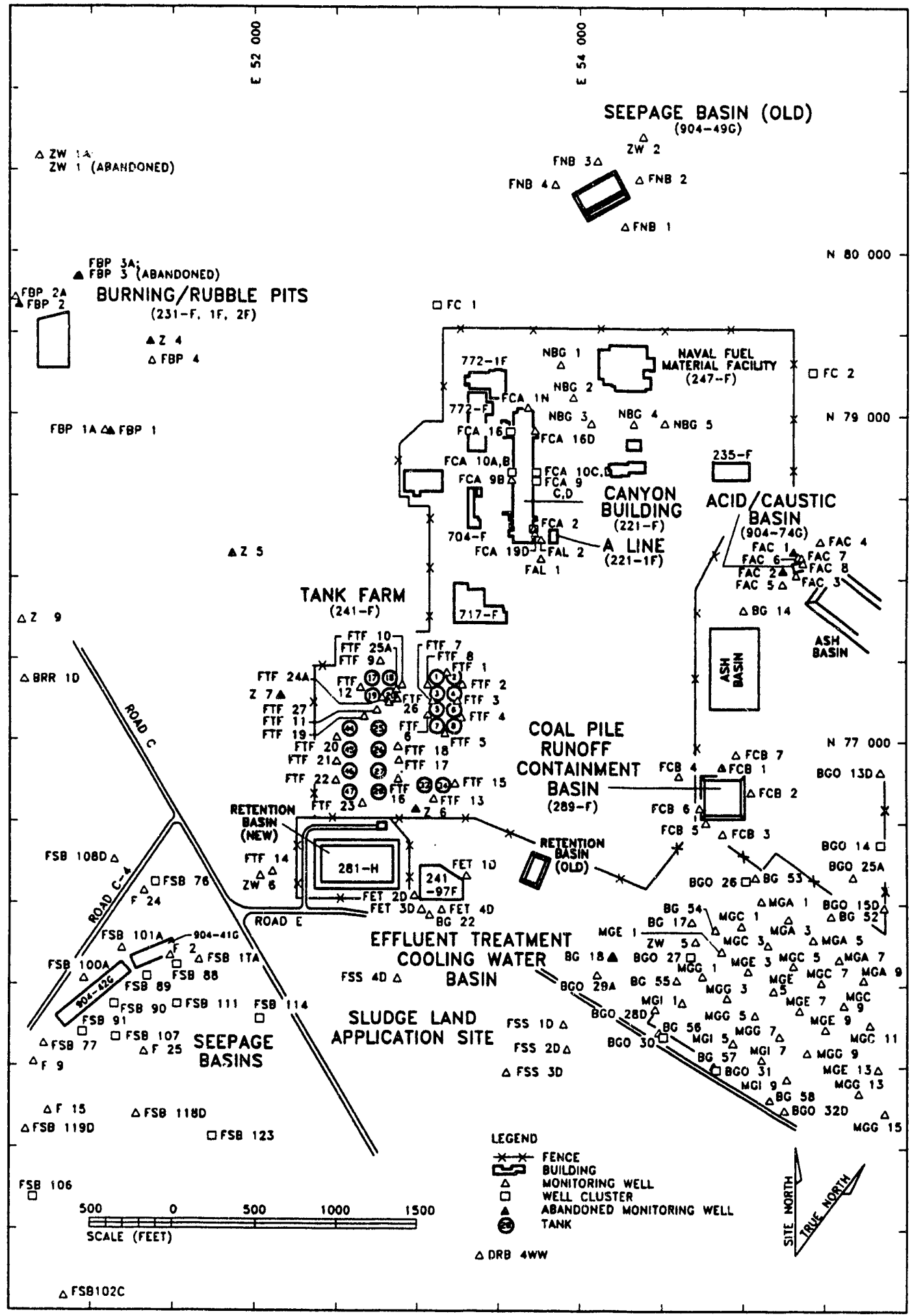

Figure A-12. Groundwater Monitoring Wells Near F-Area Canyon Building and F-Area Tínk Farm 


\section{Area Maps}

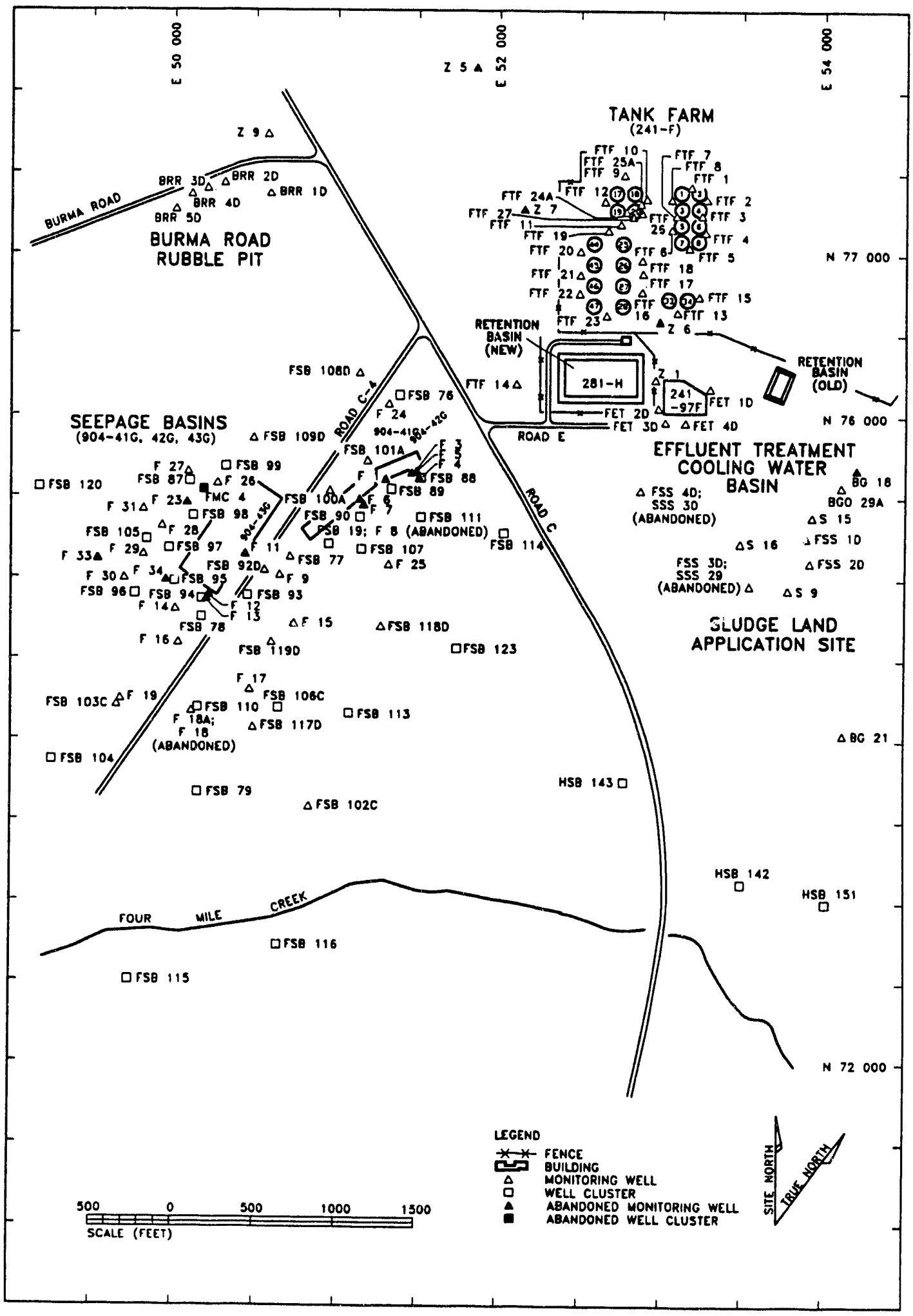

Figure A-13. Groundwater Monitoring Wells Near F-Area Seepage Basins and F-Area Tank Farm 


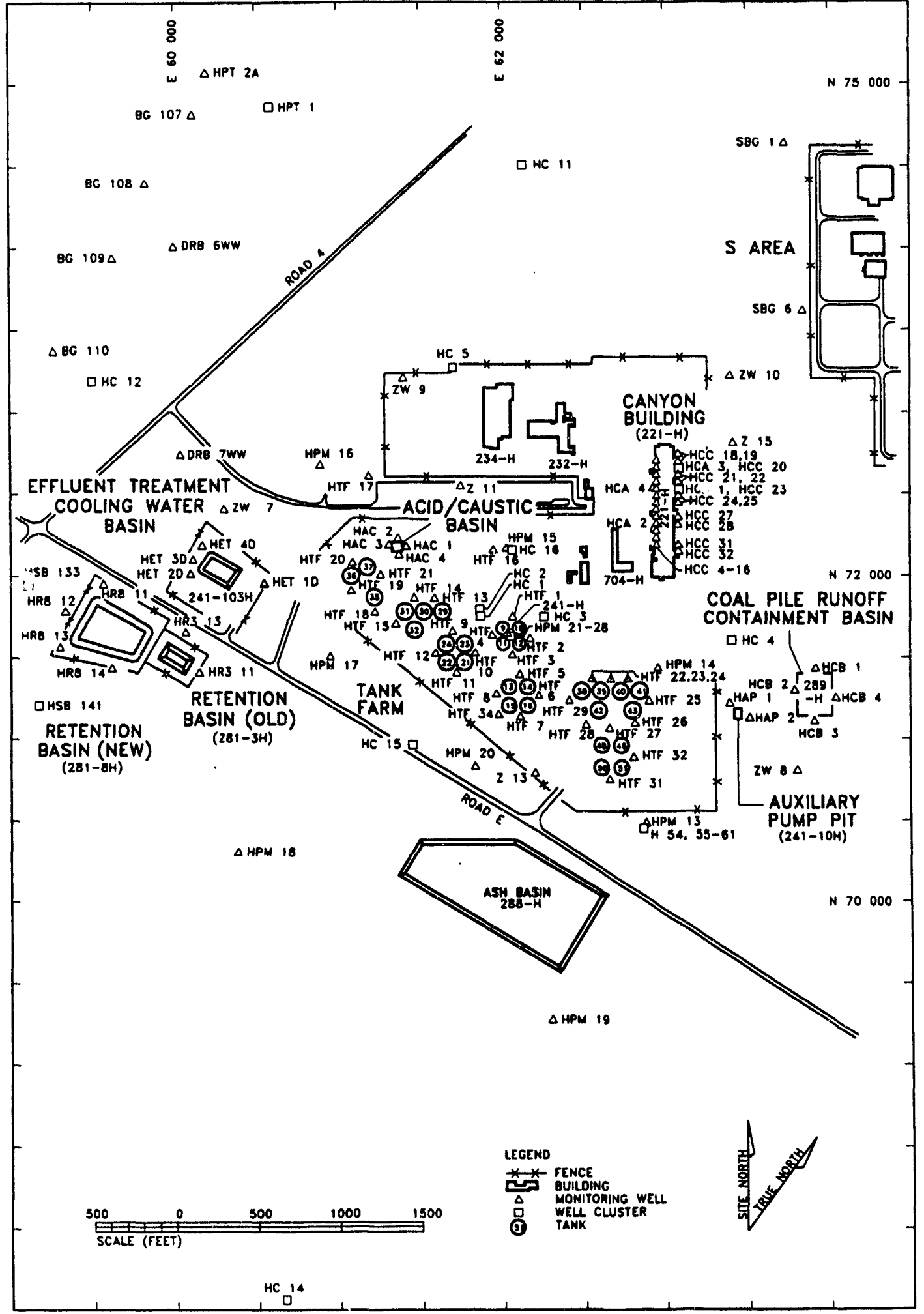

Figure A-14. Active Groundwater Monitoring Wells at the Central Part of $\mathrm{H}$ Area 


\section{Area Maps}

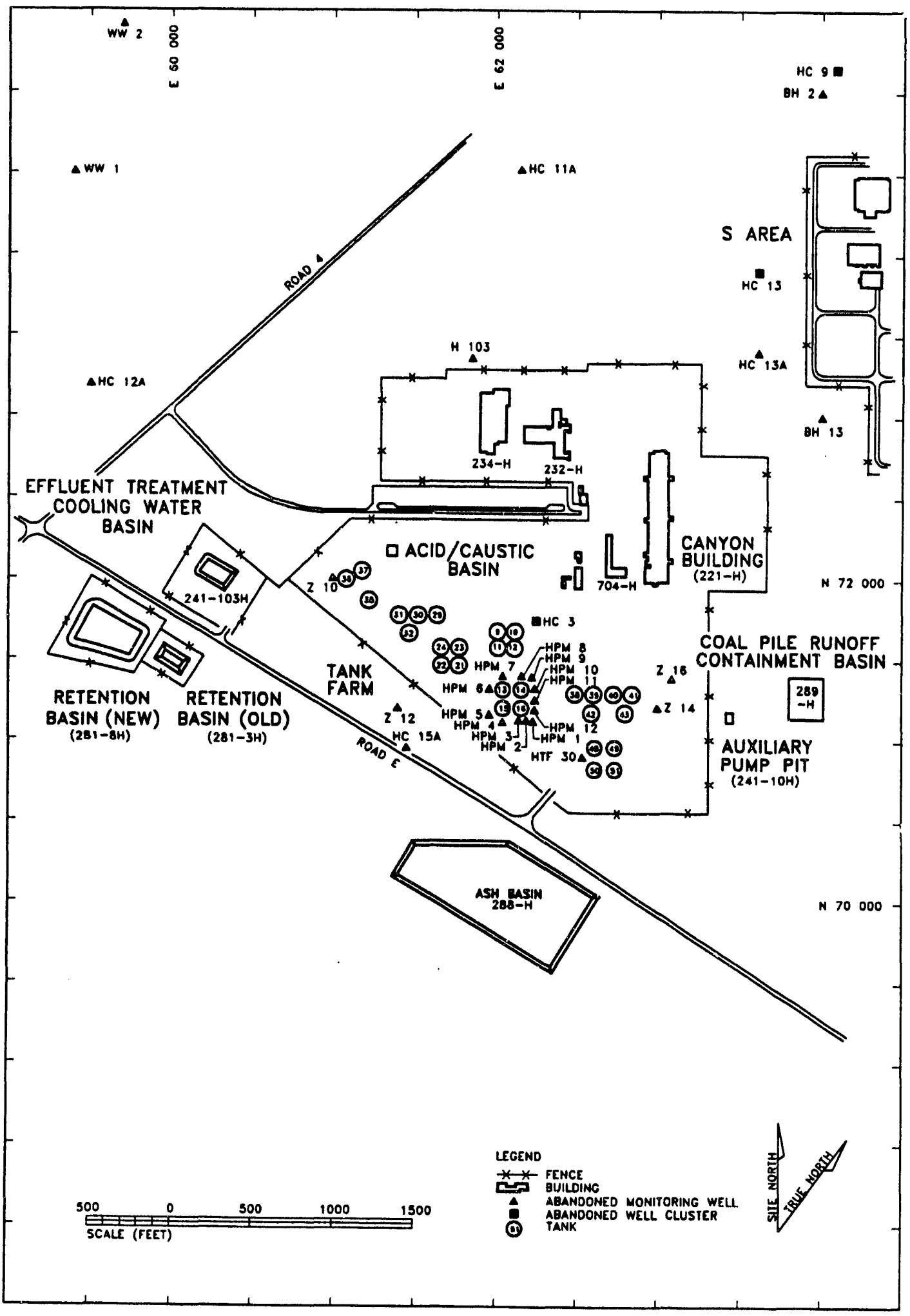

Figure A-15. Abandoned Groundwater Monitoring Wells at the Central Part of H Area 


\section{Area Maps}

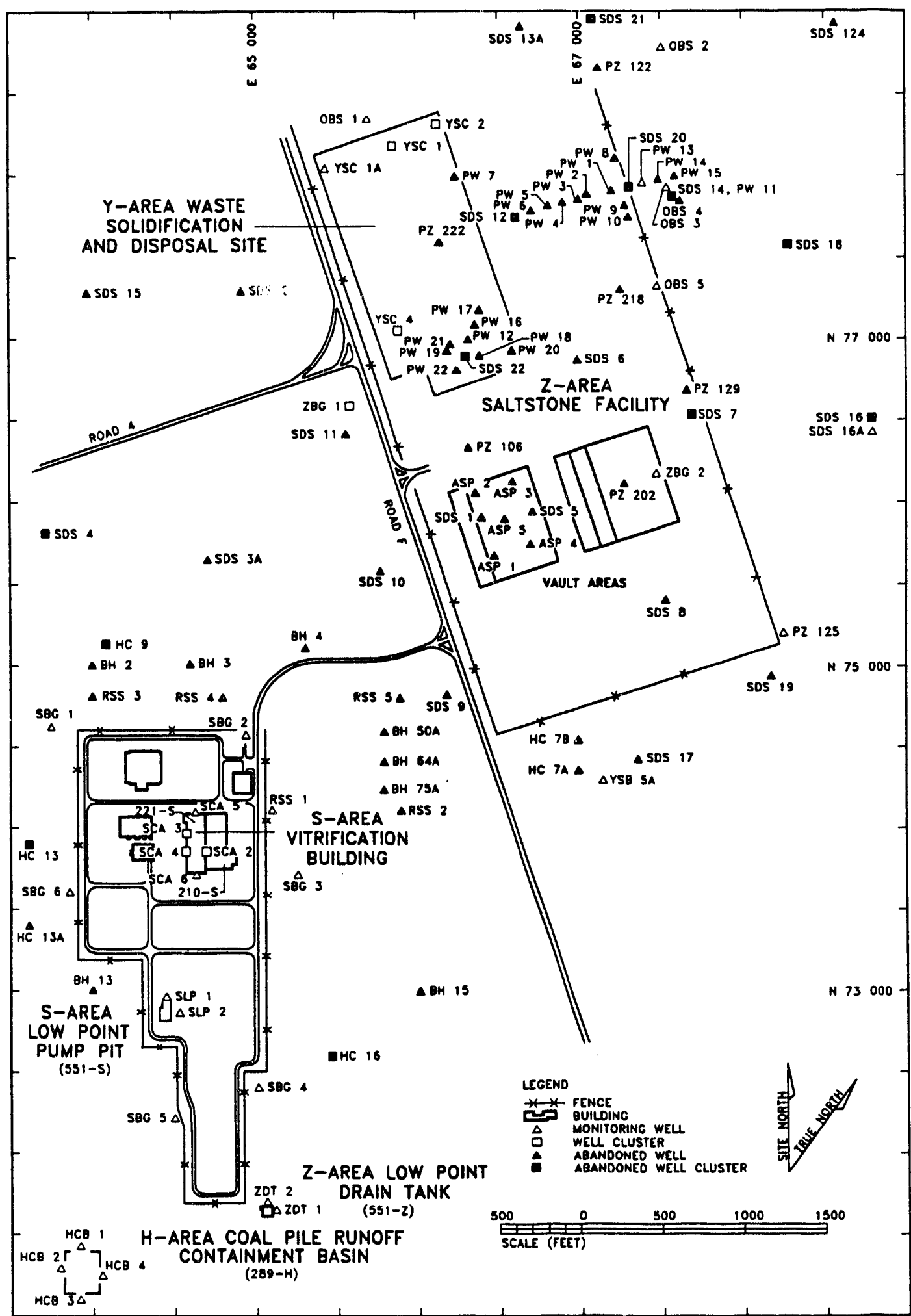

Figure A-16. Groundwater Monitoring Wells at the North Part of H Area, at S Area, Y Area, Z Area, and Vicinity 


\section{Area Maps}

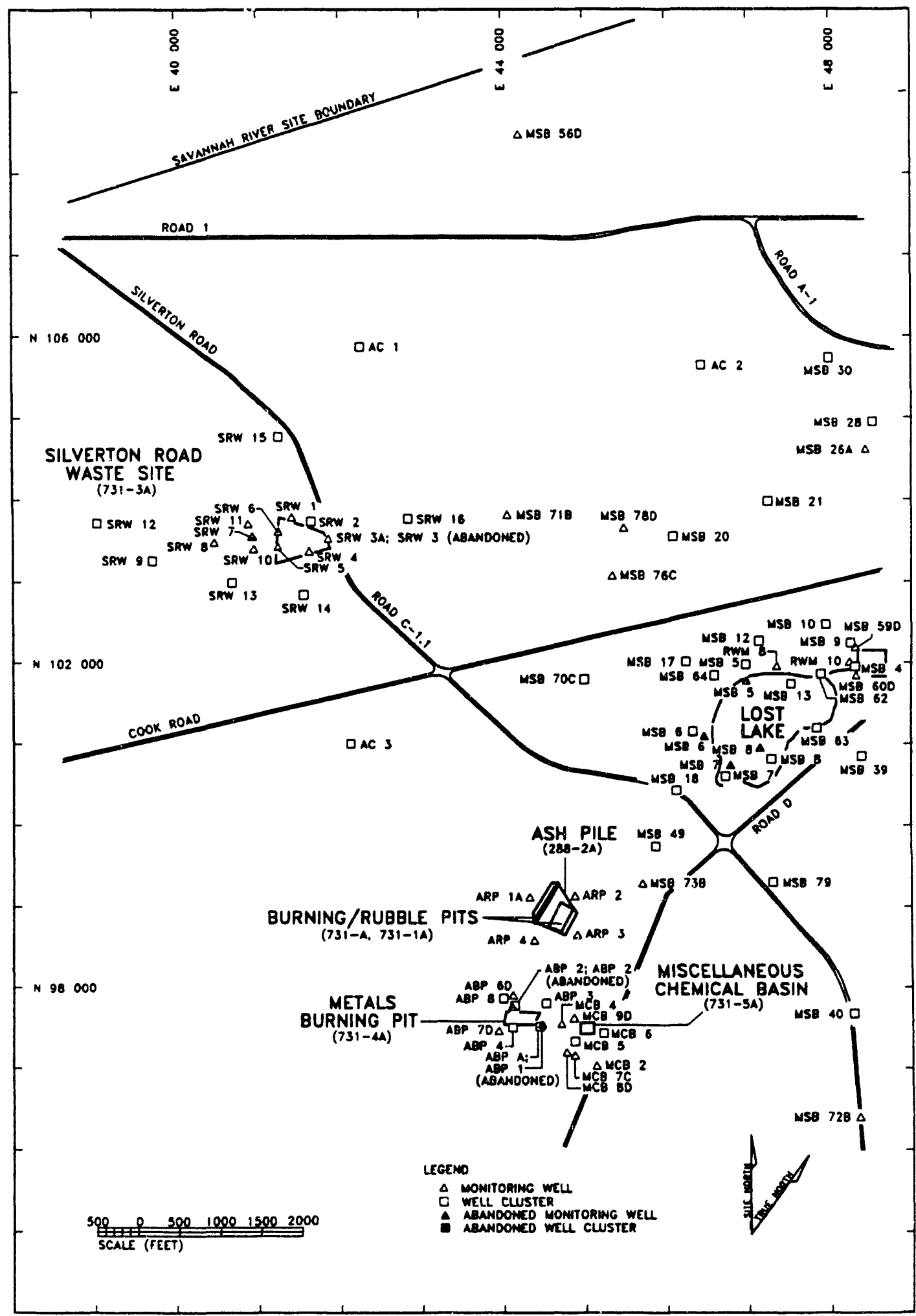

Figure A-17. Groundwater Monitoring Wells at $A$ and $M$ Areas and Silverton Road Waste Site 


\section{Area Maps}

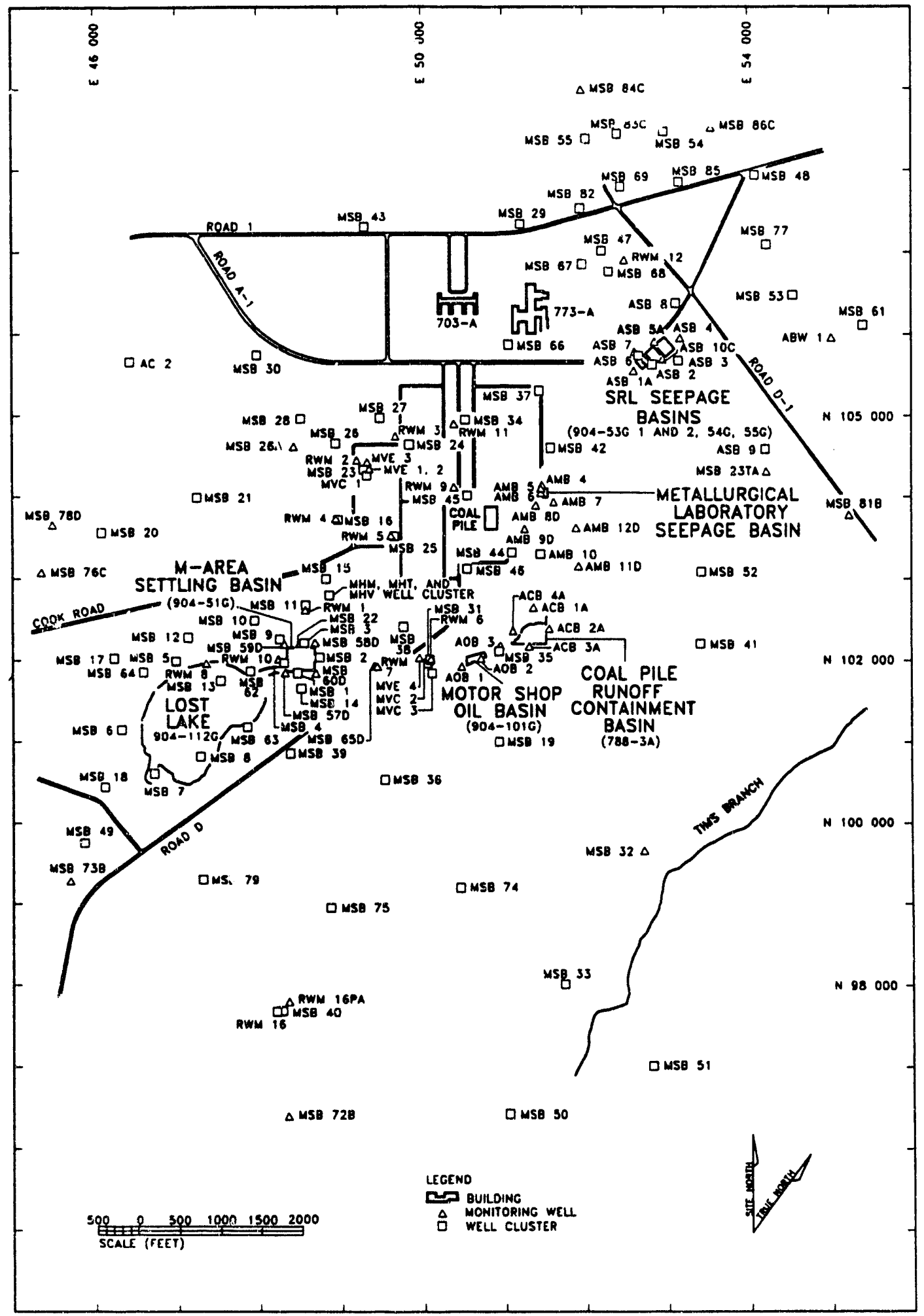

Figure A-18. Active Groundwater Monitoring Wells at $\mathrm{A}$ and $\mathrm{M}$ Areas 


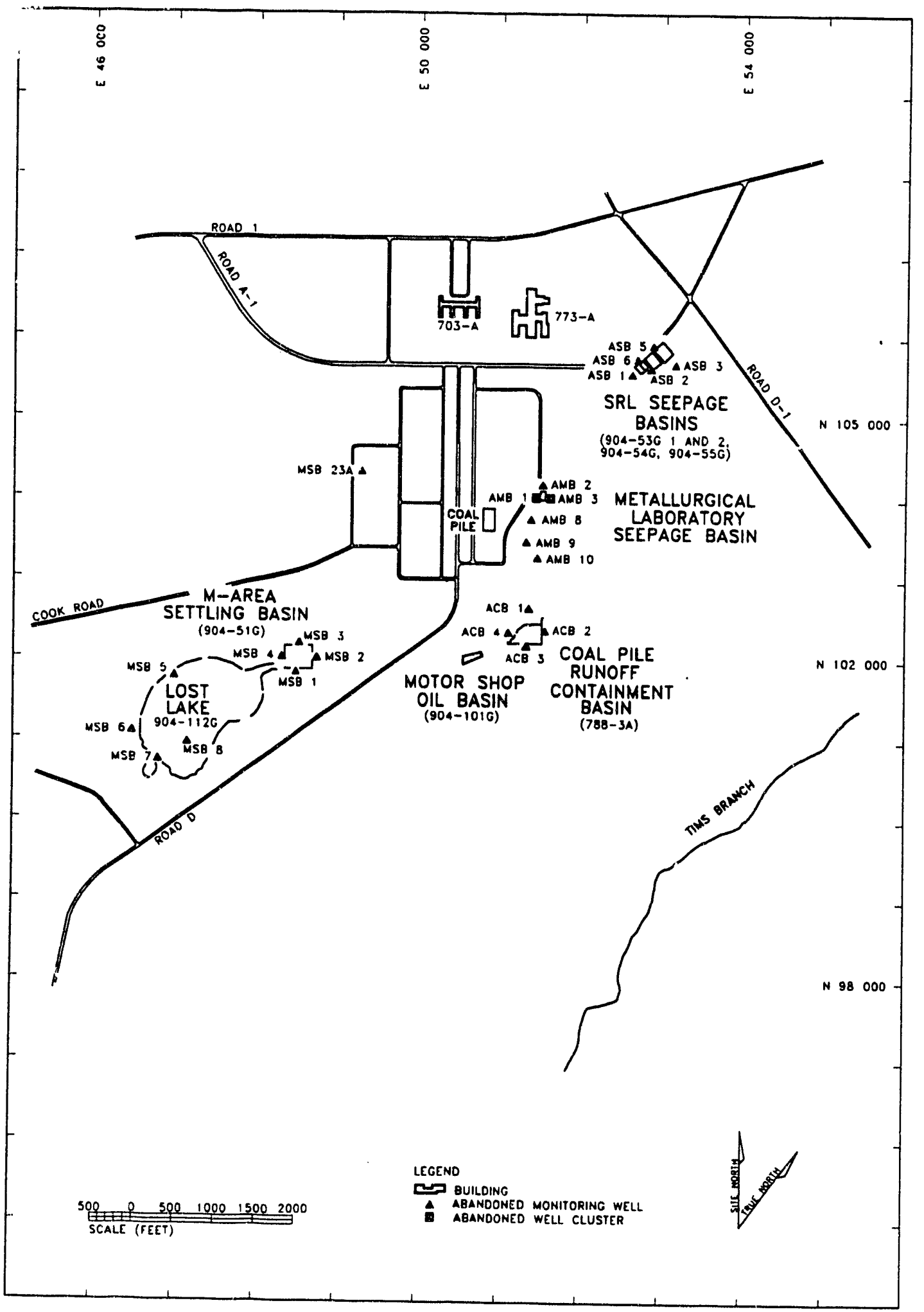

Figure A-19. Abandoned Groundwater Monitoring Wells at A and M Areas 


\section{Area Maps}

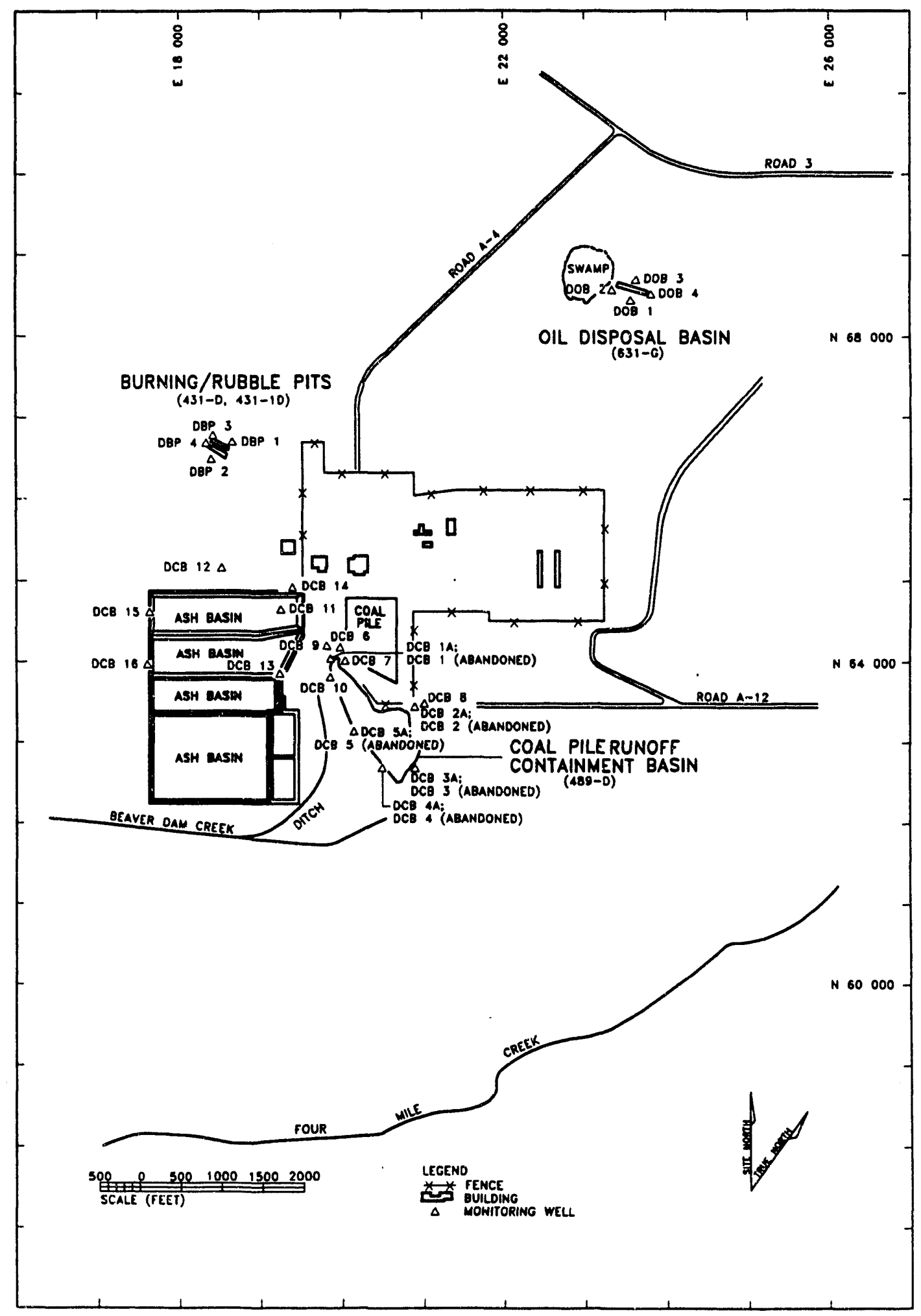

Figure A-20. Groundwater Monitoring Wells at D Area 


\section{Area Maps}

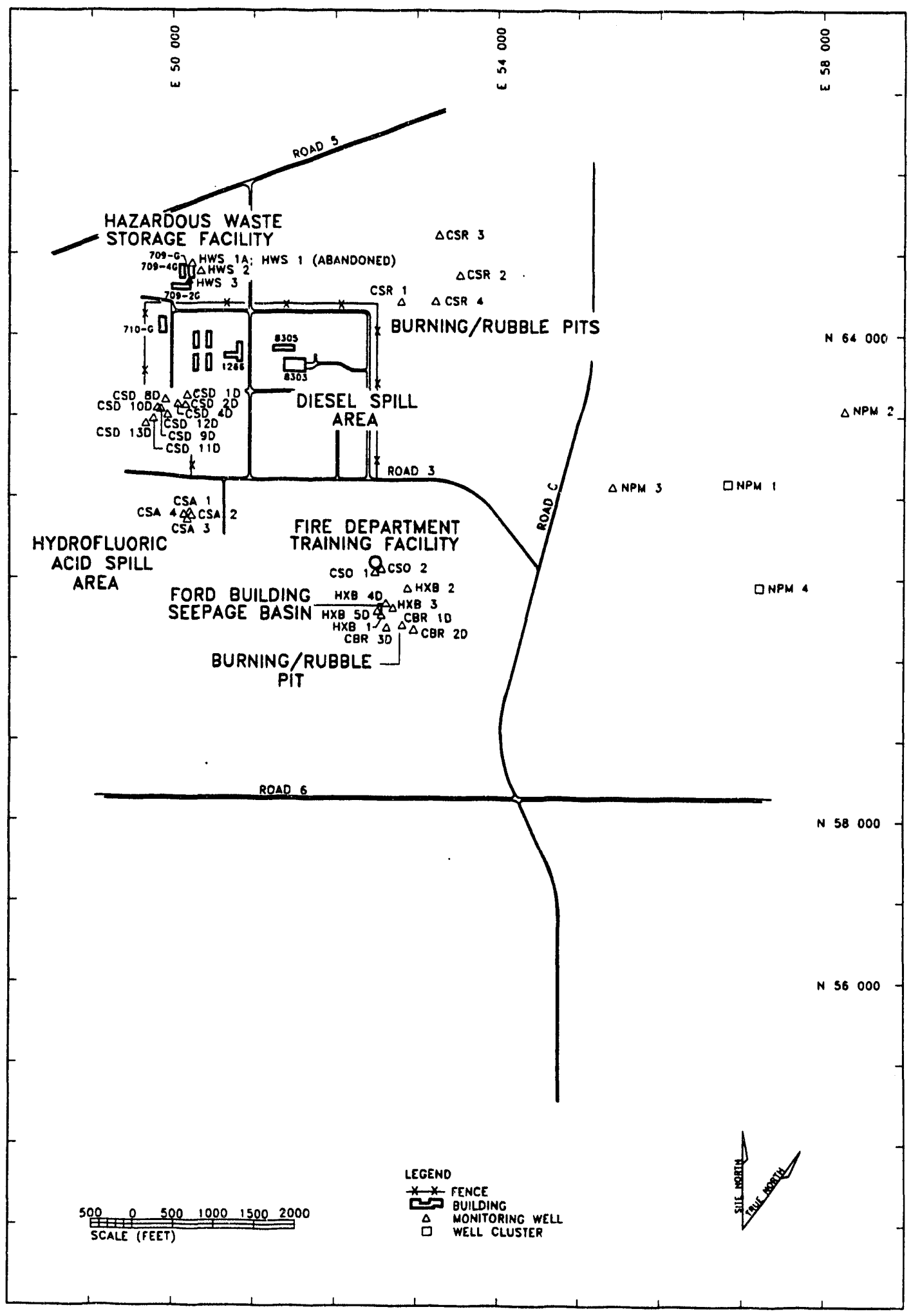

Figure A-21. Groundwater Monitoring Wells at and Near the Central Shops Area and the Hydrofluoric Acid Spill Area 


\section{Area Maps}

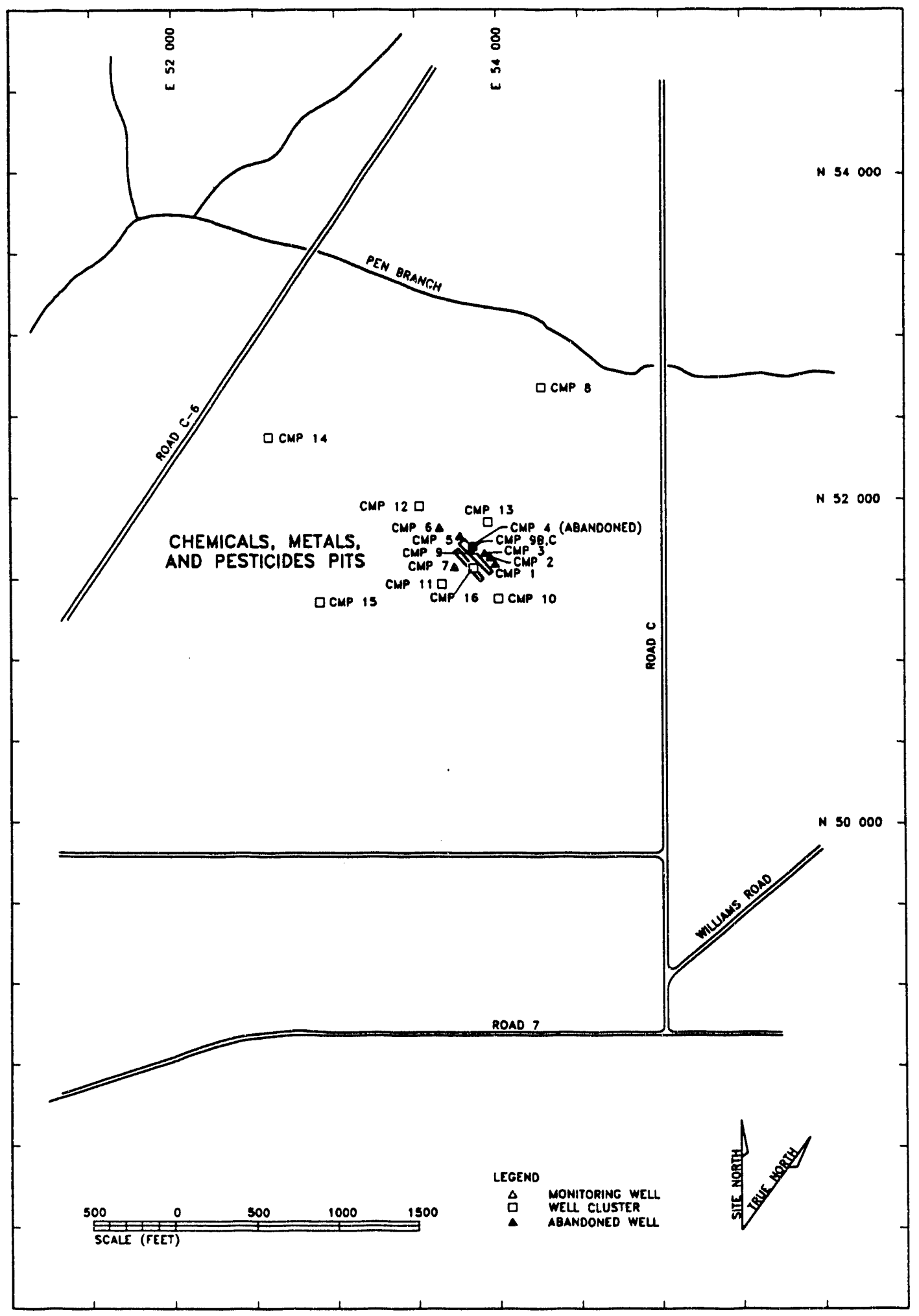

Figure A-22. Groundwater Monitoring Wells at the Chemicals, Metals, and Pesticides Pits 


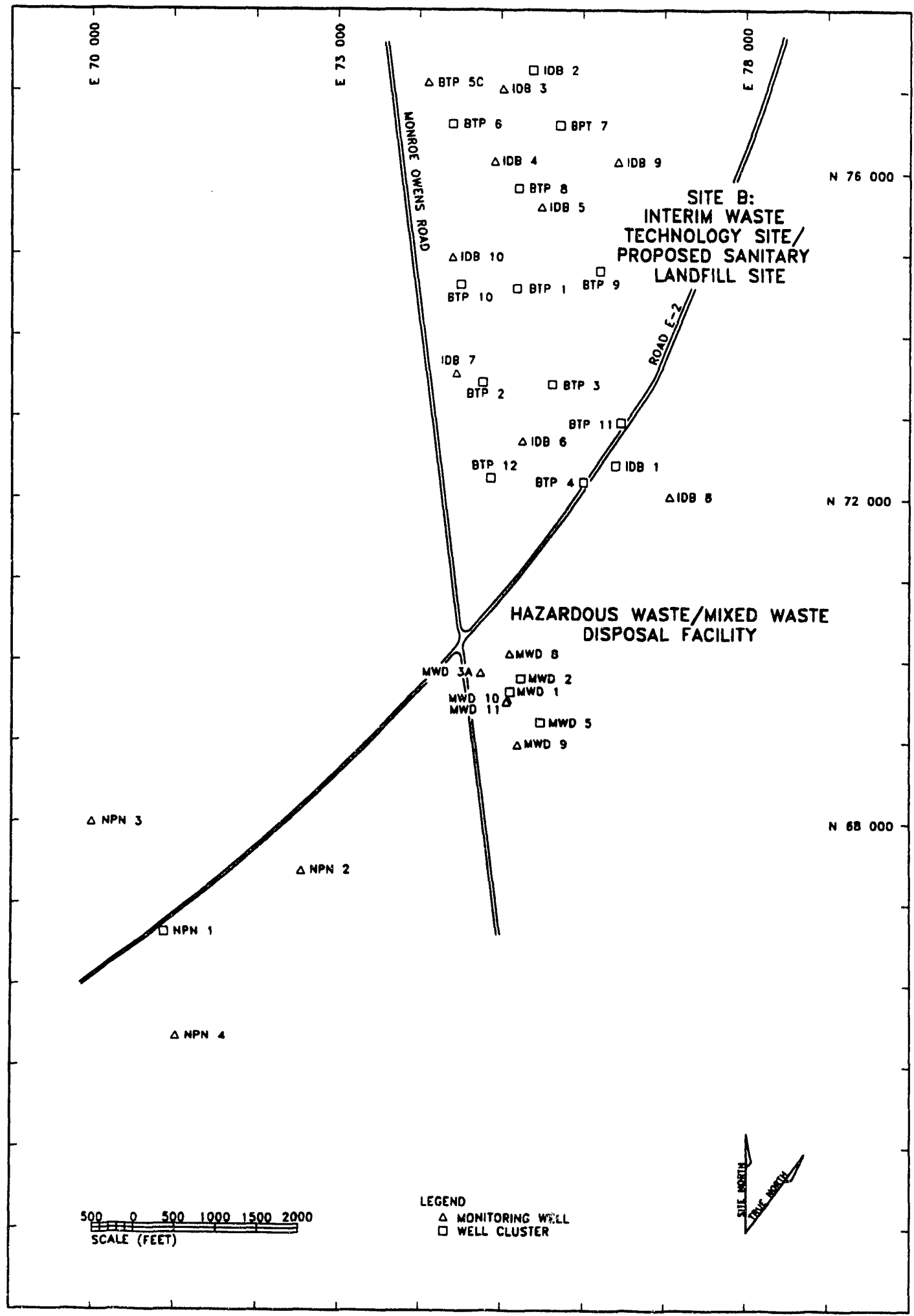

Figure A-23. Groundwater Monitoring Wells at Site B (Interim Waste Technology Site/Proposed Sanitary Landfill Site) 


\section{Area Maps}

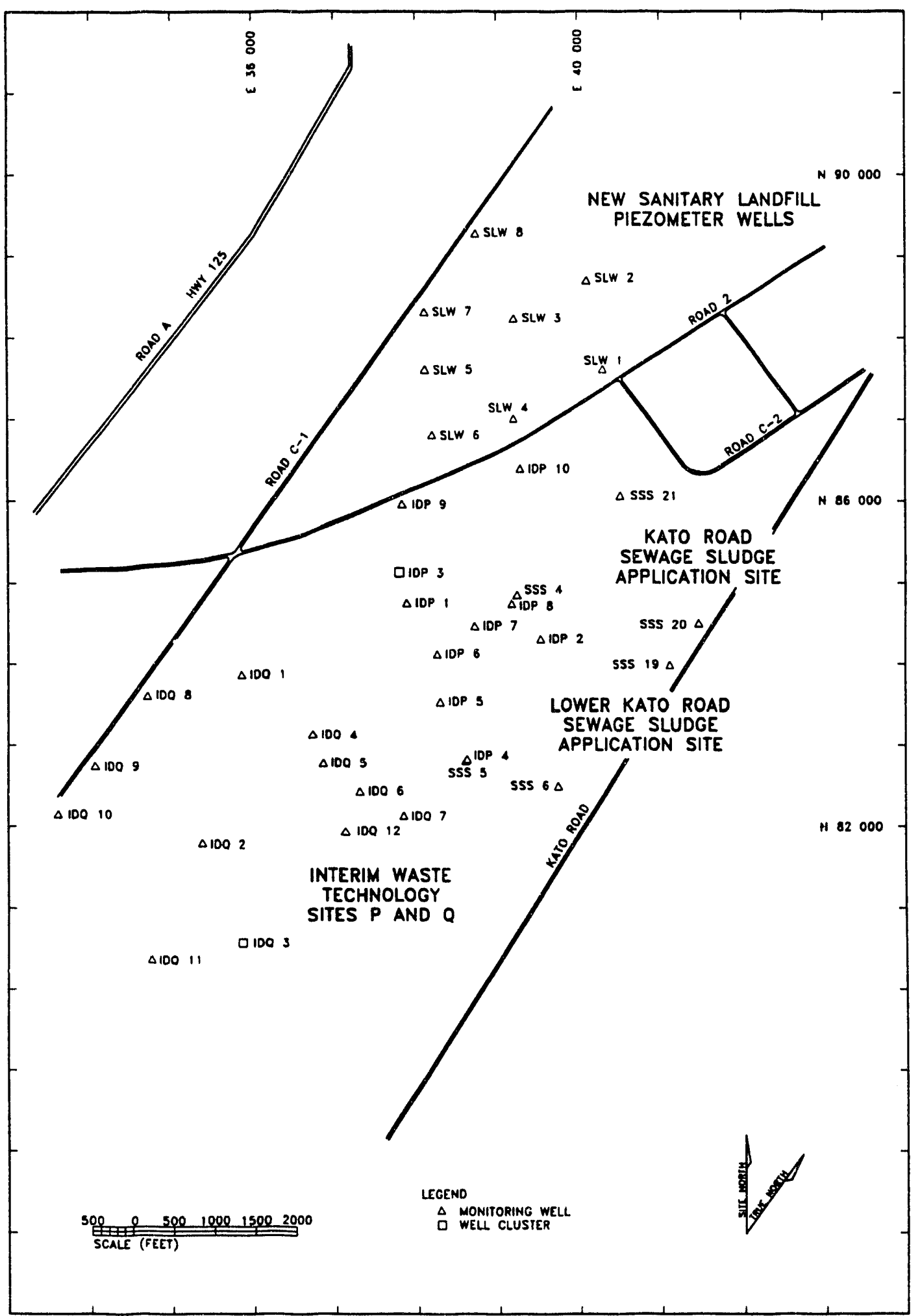

Figure A-24. Groundwater Monitoring Wells at Sites P and Q (Interim Waste Technology Sites), the New Sanitary Landfill, and the Kato Road and Lower Kato Road Sewage Sludge Application Sites 


\section{Area Maps}

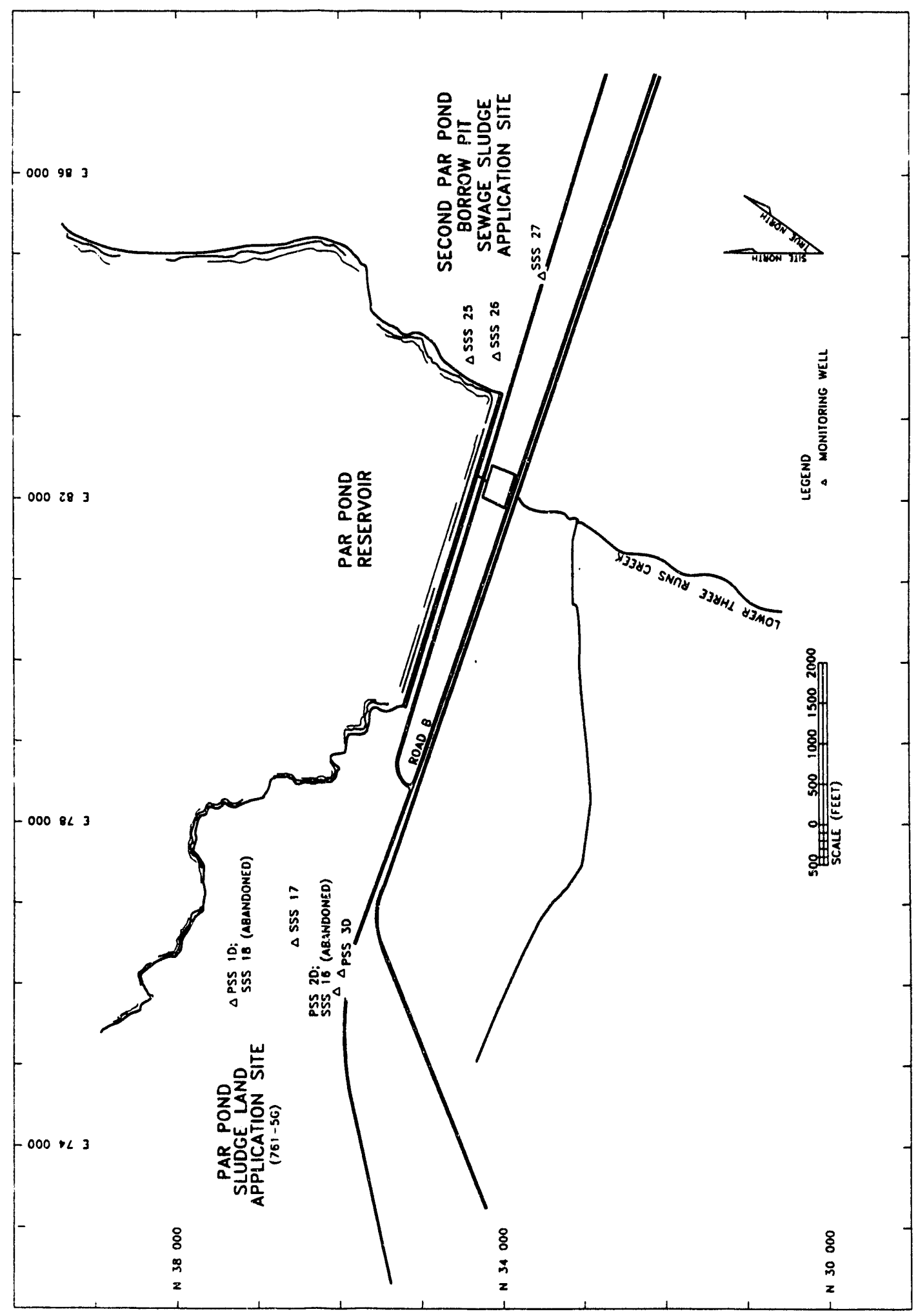

Figure A-25. Groundwater Monitoring Wells at Par Pond 


\section{Area Maps}

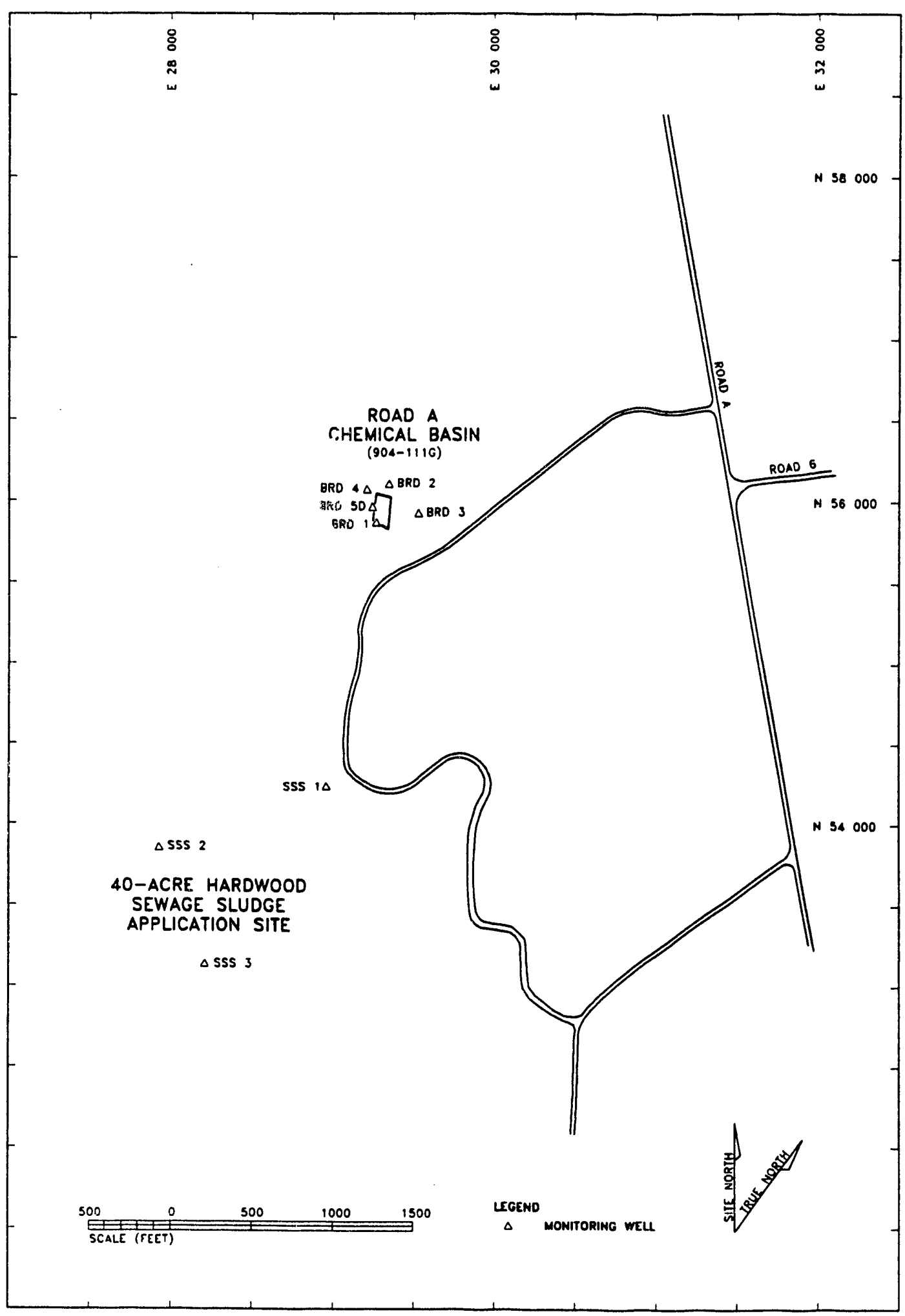

Figure A-26. Groundwater Monitoring Wells at Road A Chemical Basin and 40-Acre Hardwood Sewage Sludge Application Site 


\section{Area Maps}

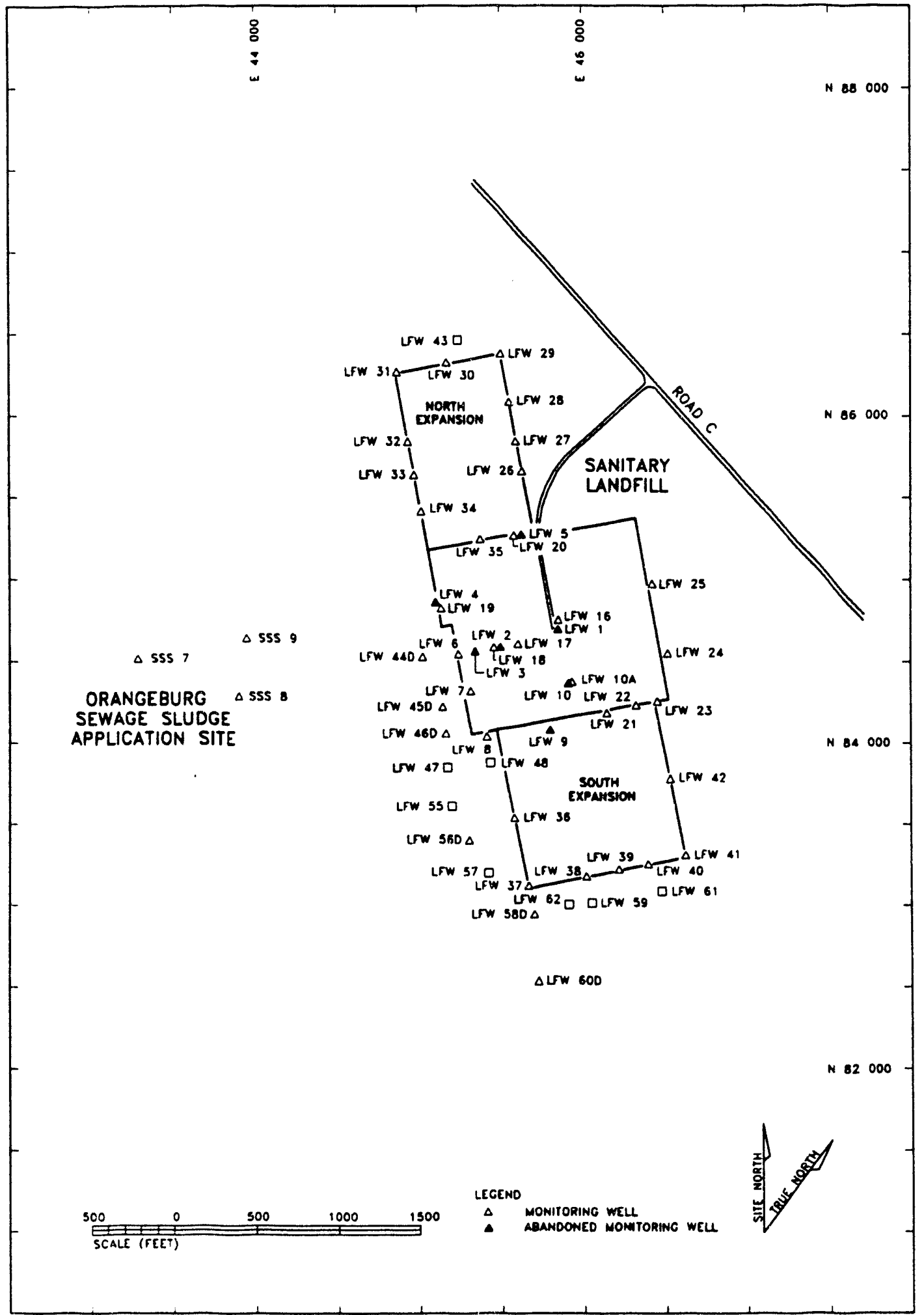

Figure A-27. Groundwater Monitoring Wells at the Sanitary Landfill and the Orangeburg Sewage Sludge Application Site 


\section{Area Maps}

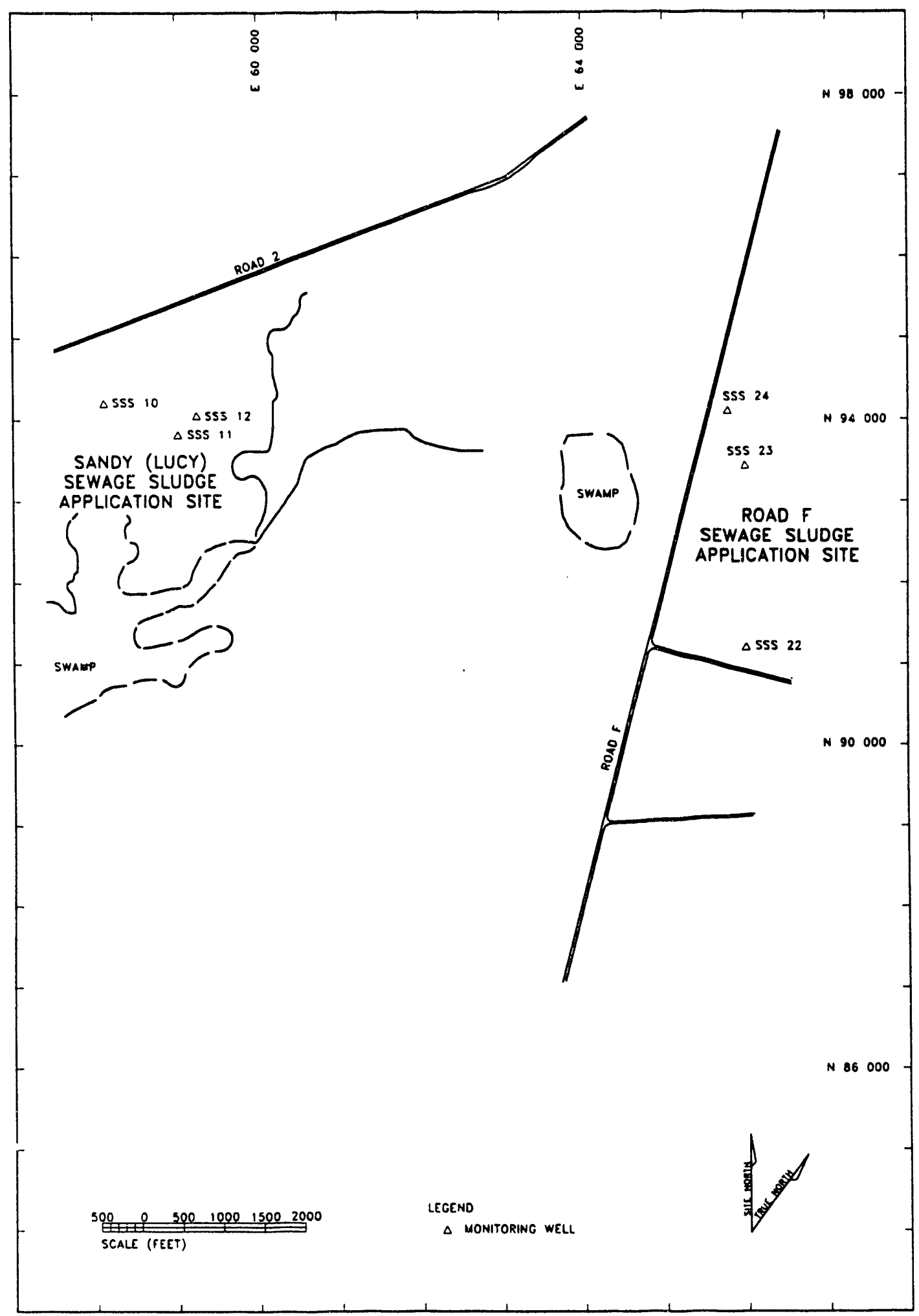

Figure A-28. Groundwater Monitoring Wells at Sandy (Lucy) and Road F Sewage Sludge Application Sites 


\section{Area Maps}

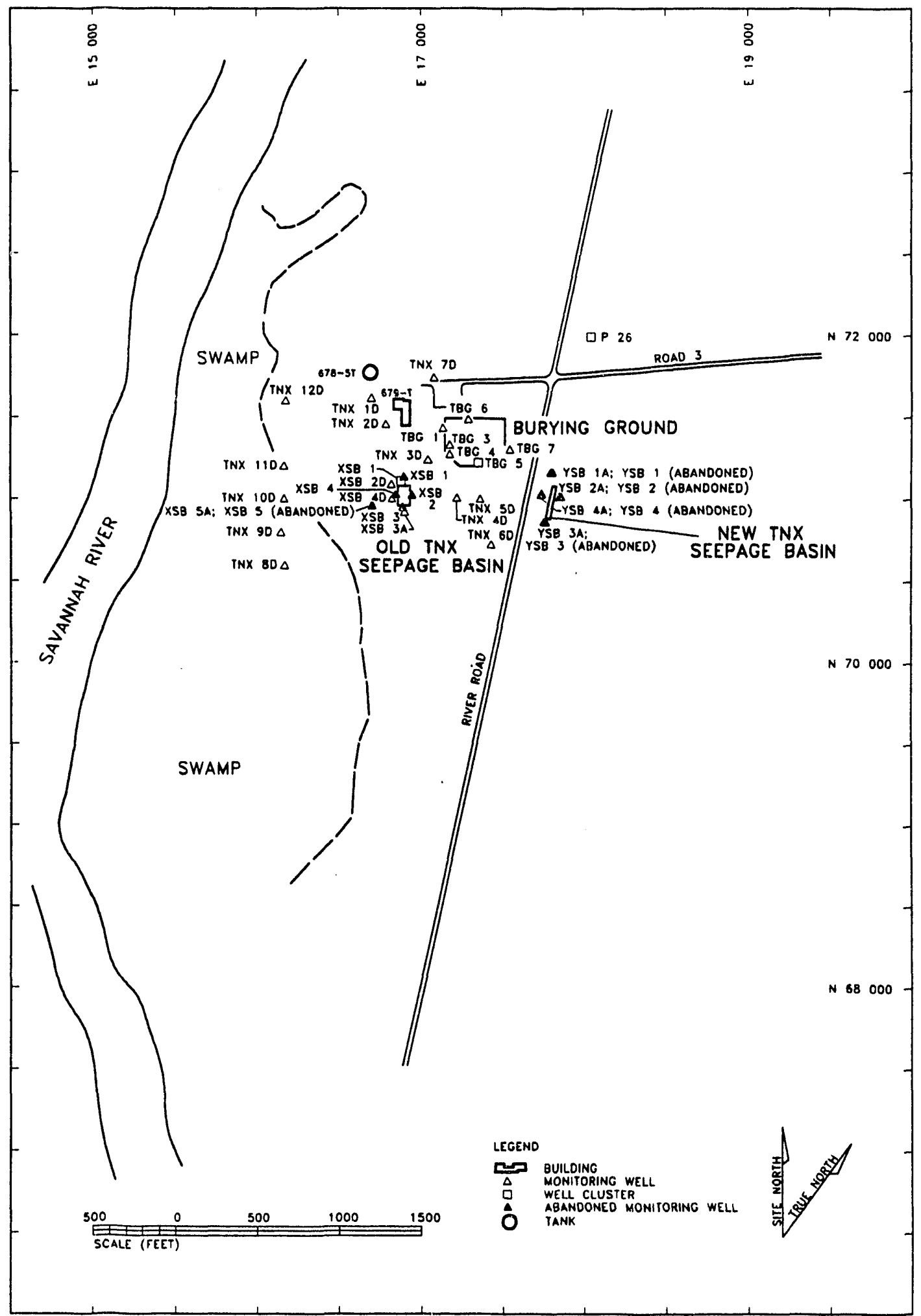

Figure A-29. Groundwater Monitoring Wells at TNX Area 

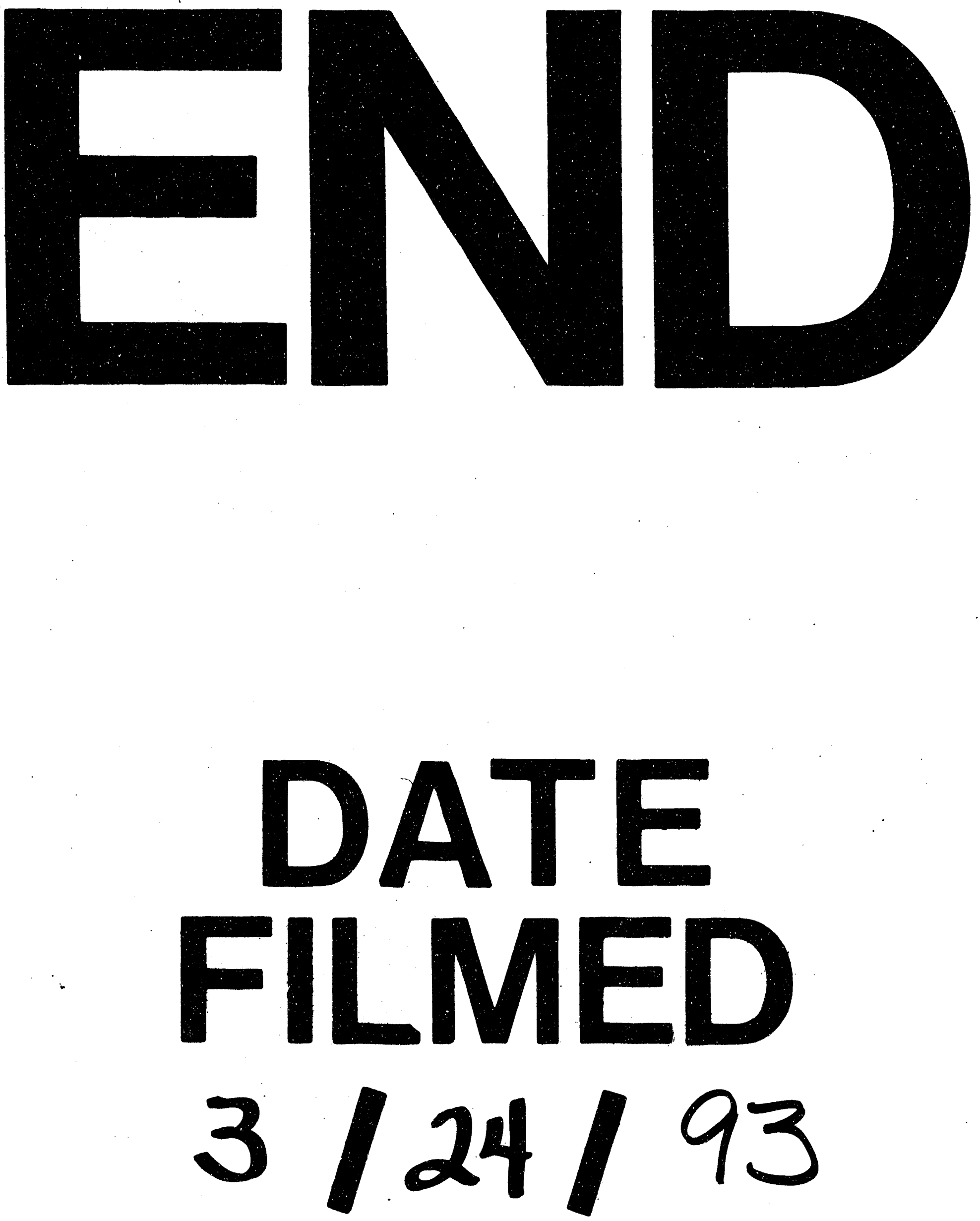
